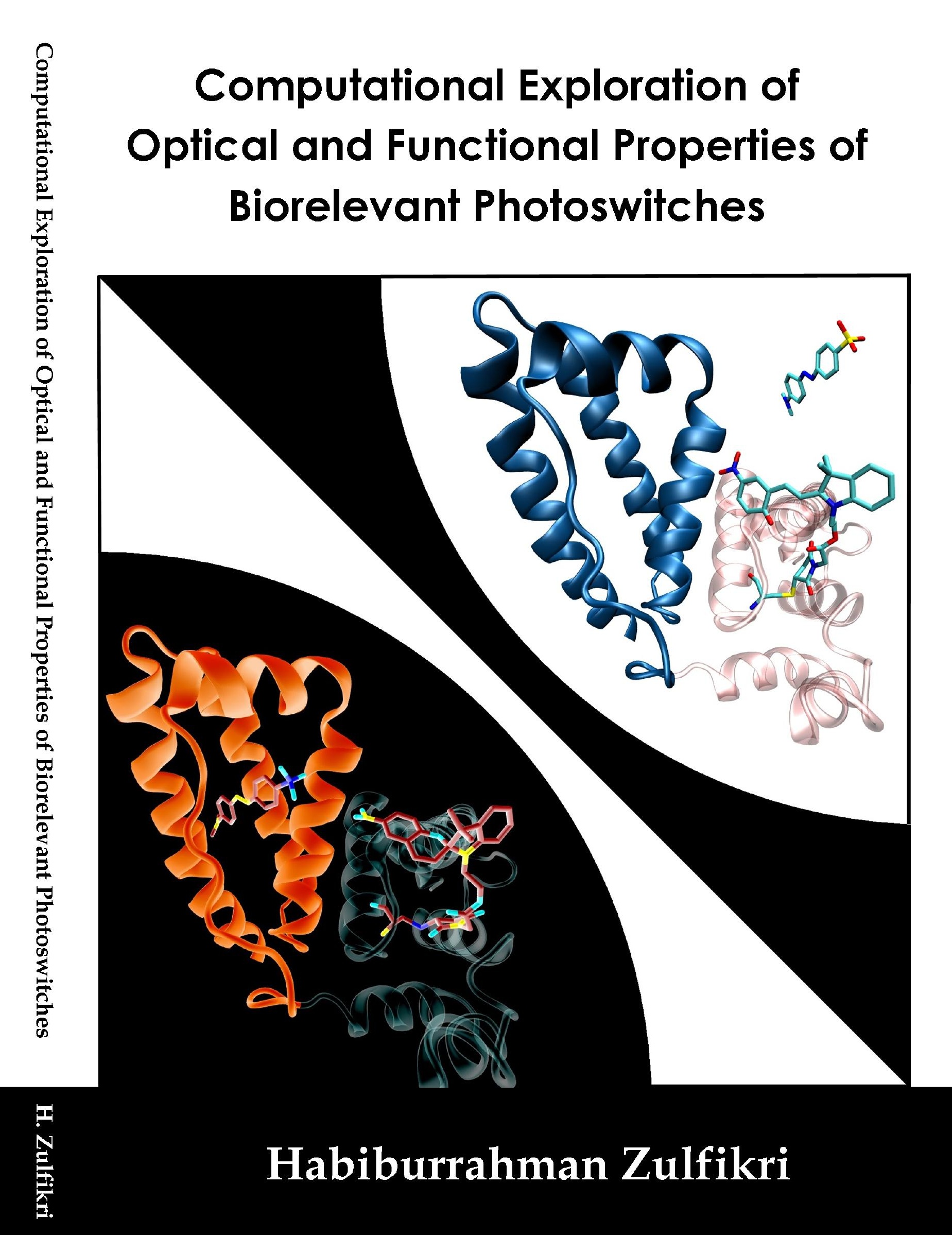


COMPUTATIONAL EXPLORATION OF OPTICAL AND FUNCTIONAL PROPERTIES OF BIORELEVANT PHOTOSWITCHES

Habiburrahman Zulfikri 
Promotion committee:

Prof. dr. ir. J. W. M. Hilgenkamp University of Twente, Chairman

Prof. dr. ir. J. W. M. Hilgenkamp University of Twente, Secretary

Prof. dr. C. Filippi

University of Twente, Supervisor

Prof. dr. W. J. Briels

University of Twente

Prof. dr. G. H. L. A. Brocks

University of Twente

Prof. dr. R. Broer

University of Groningen

Prof. dr. W. J. Buma

University of Amsterdam

Dr. R. Nifosì

NEST - CNR Istituto Nanoscienze

Computational exploration of optical and functional properties of biorelevant photoswitches

Ph.D. Dissertation, University of Twente, Enschede, The Netherlands.

ISBN: 978-90-365-4582-2

Copyright (C) 2018 by Habiburrahman Zulfikri

No part of this work may be reproduced by print, photocopy or any other means without the permission in writing from the author.

DOI: $10.3990 / 1.9789036545822$

Online version: https://doi.org/10.3990/1.9789036545822

Habiburrahman Zulfikri

h.zulfikri@outlook.com

Printed by: Ipskamp Printing - https://proefschriften.net 


\section{COMPUTATIONAL EXPLORATION OF OPTICAL AND FUNCTIONAL PROPERTIES OF BIORELEVANT PHOTOSWITCHES}

\section{DISSERTATION}

to obtain

the degree of doctor at the University of Twente, on the authority of the rector magnificus,

Prof. dr. T. T. M. Palstra,

on account of the decision of the graduation committee, to be publicly defended on Friday, $13^{\text {th }}$ of July 2018 at $12: 45$

by

Habiburrahman Zulfikri

born on November 26, 1989

in Bukittinggi, Indonesia 
This doctoral dissertation is approved by:

Prof. dr. C. Filippi

Promotor 



\section{Contents}

1 General introduction $\quad 1$

1.1 Modeling photo-induced processes . . . . . . . . . . . . . . 1

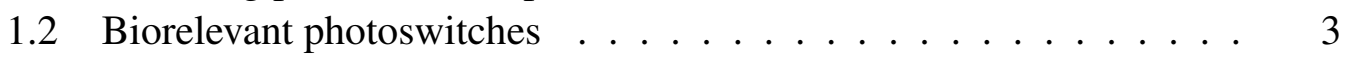

1.2.1 Natural photoswitches . . . . . . . . . . . . 3

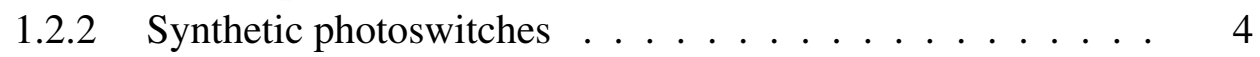

1.3 This dissertation . . . . . . . . . . . . . . 8

2 Theoretical methods $\quad \mathbf{1 5}$

2.1 Quantum mechanical calculations ... . . . . . . . . . 15

2.1.1 Single-determinant wave function . . . . . . . . . . 15

2.1.2 Correlation methods . . . . . . . . . . . . . . . 17

2.1.3 Quantum Monte Carlo methods . . . . . . . . . . . . 19

2.1.4 Density functional theory . . . . . . . . . . . . 22

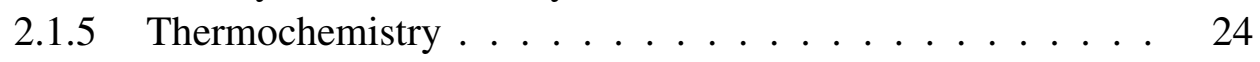

2.2 Molecular mechanical simulations . . . . . . . . . . . . . 27

2.2.1 Classical force field . . . . . . . . . . . . . . 27

2.2 .2 Molecular dynamics . . . . . . . . . . . 30

2.3 Computational details . . . . . . . . . . . . . . . 32

3 Multiple-resonance local wave functions for accurate excited states in QMC 39

3.1 Introduction . . . . . . . . . . . . . . 40

3.2 Method ............................. 41

3.2.1 Domains and excitation classes ........... . . . . . 43

3.2.2 Beyond one resonance structure . . . . . . . . . . . 48

3.2.3 Orbital optimization . . . . . . . . . . . . 51

3.3 Computational details . . . . . . . . . . . . . . . . . . . . 52

3.4 Results and discussions . . . . . . . . . . . . . . . . . . 54

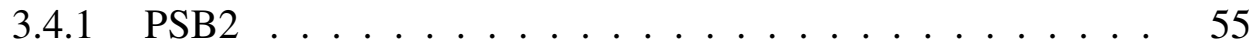

$3.4 .2 \quad$ PSB $3 \ldots \ldots \ldots \ldots \ldots \ldots$

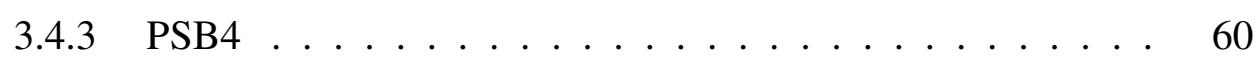

3.4 .4 Geometry optimization . . . . . . . . . . . . 61

3.5 Conclusions . . . . . . . . . . . . . . . 62

3.6 Appendix: Domains of larger retinal models . . . . . . . . . . 65 
4 Photo-induced mechanism of isomerization of DASA 73

4.1 Introduction . . . . . . . . . . . . . . . . . . . 74

4.2 Computational details . . . . . . . . . . . . . . 75

4.3 Results and discussions . . . . . . . . . . . . . . 76

4.3.1 Thermal step of DASA-1 . . . . . . . . . . 76

4.3.2 Thermal step of DASA-2 . . . . . . . . . . . 84

4.3 .3 Actinic step . . . . . . . . . . . . . . . 85

4.4 Conclusions . . . . . . . . . . . . . . . . . . . . 90

4.5 Appendix: Other functionals . . . . . . . . . . . . . . 92

5 Regulating allosteric interactions in a protein hybrid with light 99

5.1 Introduction . . . . . . . . . . . . . . . . 100

5.2 Experimental background . . . . . . . . . . . . . . 101

5.3 Computational details . . . . . . . . . . . . . . . . . 103

5.3.1 Molecular docking ................ 103

5.3.2 Molecular dynamics simulation . . . . . . . . . . . . 104

5.4 Results and discussions . . . . . . . . . . . . . . . 105

5.4.1 Nature of interaction between HSA and photoswitch . . . . 105

5.4.2 Mechanistic insight on ligand release . . . . . . . . . . . . 109

5.5 Conclusions . . . . . . . . . . . . . . . . . . . 117

5.6 Appendix: Force-field construction . . . . . . . . . . . . . . 118

$\begin{array}{ll}\text { Summary } & 131\end{array}$

$\begin{array}{ll}\text { Samenvatting } & 133\end{array}$

$\begin{array}{ll}\text { List of publications } & 134\end{array}$

$\begin{array}{ll}\text { Acknowledgements } & 137\end{array}$ 


\section{Chapter 1}

\section{General introduction}

In recent years, dynamic molecules, whose structures and functions can be switched between different states, have been considerably developed and studied experimentally and theoretically [1-4]. As opposed to static systems, dynamic molecules isomerize in response to external triggers yielding products which are usually different in size and properties from the initial isomer. When the external trigger is light, dynamic molecules are called photoswitches. Light is by far the most promising tool for manipulating molecules [1-6] as one can choose a specific wavelength to tune the reaction with high spatial and temporal precision. Moreover, no chemical contaminants are introduced into the system, which renders the interpretation of the process easier and also ensures no significant additional perturbation upon switching.

\subsection{Modeling photo-induced processes}

Modeling the photo-induced behavior of photoswitches requires the availability of robust approaches able to describe the excited states of relatively large molecules (about 40 atoms for the molecules studied in this dissertation) as well as their coupling with the nuclear degrees of freedom in the dynamical process. When thinking about electronic structure calculations for large systems, density functional theory and its extension to excited states, time-dependent density functional theory (TDDFT), immediately come to mind as the most natural choice, being computationally affordable for large molecules [7]. It is however well documented that approximations may severely limit the accuracy of TDDFT, in particular for excitations characterized by charge-transfer or multi-reference character as in the vicinity of conical intersections [8]. Furthermore, benchmarking of TDDFT has mostly focused on vertical electronic excitations, and relatively little is known on the performance of TDDFT outside the Franck-Condon region, with the available studies revealing for instance a rather uneven quality in the optimal excited-state structures [9-11].

These shortcomings can in principle be overcome by using correlated approaches and multiconfigurational wave function methods such as the complete-active-space self-consistent-field (CASSCF) method [12], which is the most prominent technique used in the exploration of excited-state potential energy surfaces (PES). In these sim- 
ulations, the interatomic gradients are computed at the CASSCF level, and the energies are usually further refined by including perturbative corrections. While multiconfigurational wave function methods have been often successfully used to study the photochemistry of dynamic molecules, the results can be strongly dependent on the choice of orbitals used in the active space (as we will also show in this dissertation), and as the system becomes larger, one is anyhow limited to relatively small expansions. Both static and dynamic correlations could be more robustly described by perturbation approaches but these techniques can only be applied to small- and medium-size systems as their computational cost becomes very quickly prohibitive.

Alternatively, one can employ quantum Monte Carlo (QMC) techniques [13], which are a wide class of stochastic algorithms to simulate quantum many-body systems. As compared to traditional quantum chemistry approaches, solving the Schrödinger equation via Monte Carlo techniques offers important advantages: the stochastic nature of the integration allows greater flexibility in the many-body wave functions employed, which can explicitly depend on the inter-electron coordinates, and leads to a computational cost which scales favorably with system size (a mere $N^{4}$ in the number of electrons $N$ for the most widely used variational and diffusion Monte Carlo variants). Furthermore, the intrinsic parallelism of QMC algorithms renders these techniques ideal candidates to take advantage of modern parallel computers and hybrid architectures. Thanks to very recent developments in the calculation of efficient derivatives of the energy (e.g. interatomic gradients) [14], these methods represent a particularly promising tool for the accurate study of the lightinduced behavior of photoswitches. In this dissertation, we further advance QMC methodology for excited-state calculations and develop a construction scheme for accurate and compact excited-state wave functions based on local orbitals.

In addition to the difficulties of a quantum mechanical description, the environment surrounding the photoswitch should also be modeled accurately because isomerization reactions are usually strongly solvent dependent. The environment can change the absorption spectrum and also affect the reaction rate to such an extent that it can suppress the photoswitching in some cases. Moreover, in practice, photoswitches are coupled to a supporting (bio)matrix, either naturally or artificially, to achieve control or induce novel functionalities in the combined system. To account for the role of the environment, one can employ discrete multi-scale schemes where the environment is modeled classically, for example as a sea of point charges enclosing the quantum photoswitch, in so-called quantum mechanics in molecular mechanics (QM/MM) simulations. Since the charge density of the excited state can significantly differ from that of the ground state, static point charges which act as mere spectators are not a sufficiently good representation of the embedding system, and the inclusion of the response of the environment is often needed. This is possible for instance through a scheme which introduces classical induced dipoles to take into account mutual polarization effects between the quantum and classical regions [15-18]. In this dissertation work, we also contribute to the development of embedding techniques for excited states through the design of a state-specific scheme for ground- and excited-state QMC calculations in polarizable dipoles [19]. 


\subsection{Biorelevant photoswitches}

In this section, we briefly describe two categories of photoswitches relevant for this dissertation and discuss their molecular isomerization and potential application in biosystems. These photoswitches are bio-compatible as they can function in biological systems and at physiological conditions. We first focus on a prototypical natural photoswitch, which represents an ideal playground for the development and testing of novel methodologies for excited states. We then consider two synthetic photoswitches very relevant for applications and investigate how they function and affect the functioning of a complex biosystem.

\subsubsection{Natural photoswitches}

Nature commonly employs dynamic molecules (called chromophores) embedded in a protein support. One of the most familiar examples of natural chromophores is the 11-cis retinal, which is the light-sensitive component of many visual opsins, responsible for absorbing light and initiating the visual transduction process [20]. As shown in Figure 1.1, upon visible-light irradiation, the 11-cis retinal isomerizes to produce the all-trans retinal, a process which occurs on the timescale of a few hundred femtoseconds with a high quantum yield for the final photoproduct [21]. This local structural change of the chromophore inside the constrained protein pocket induces then large-scale changes in the overall structure of the protein. These structural changes result in a series of distinct intermediate and, ultimately, in the activation of signaling pathways responsible for vision.

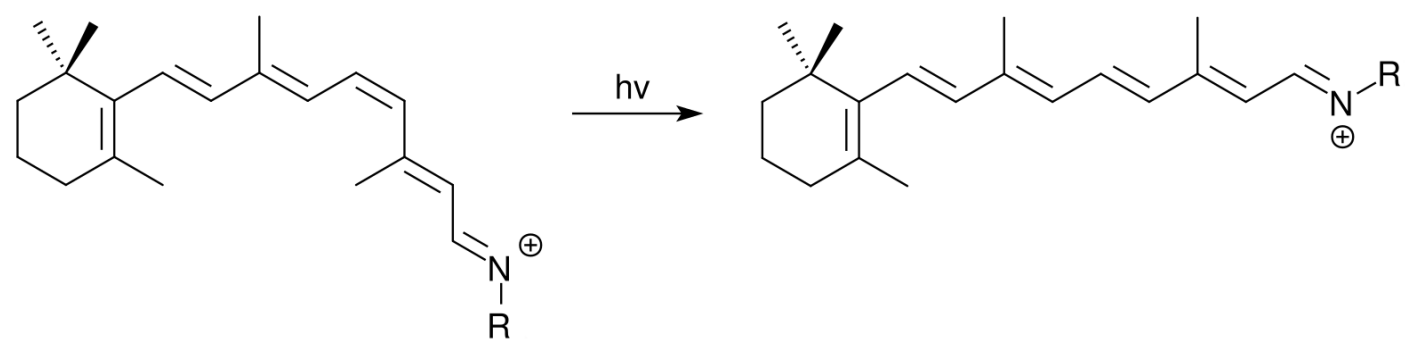

Figure 1.1: Actinic step of isomerization of the 11-cis retinal chromophore to the all-trans isomer.

The photoisomerization of the retinal chromophore has been extensively studied both experimentally and computationally. Most computational studies on the excited-state relaxation of retinal models in the gas phase, solution and protein have been performed with the CASSCF method, which predicts a strong bond inversion in the excited state followed by torsion around a formal double bond, finding favorable agreements with many experimental data [22-25]. While the chromophore is pretwisted in the protein pocket, and the preference to rotate around a particular formal double bond is therefore "programmed" in the system, highly correlated studies with CASPT2 and QMC on small retinal models have shown that the initial 
excited-state relaxation in the gas phase is more complex than the "universal" mechanism predicted by CASSCF, and is characterized by a very flexible chromophore with similar conjugated bond lengths $[26,27]$. Furthermore, a very recent investigation [28] on the minimal-energy conical intersection for the photoisomerization of the all-trans retinal chromophore in the gas phase shows that, while CASSCF yields a similar probability for rotation around the two formal carbon-carbon double bonds closer to the $\mathrm{N}$ atom, CASPT2 indicates that the photoisomerization leading to the 11-cis form is dominant consistently with experimental results [29]. All these computational investigations highlight the importance of having an approach which includes a balanced description of static and dynamical electron correlation for an accurate description of retinal and other photoswitches. Here, we take one step in this direction and, using retinal chromophore models as prototypical test systems, develop a novel compact class of many-body QMC wave functions for excited states. We demonstrate their excellent performance in computing the vertical excitation energies and optimal excited-state geometries of retinal models. In combination with the recent availability of efficient QMC gradients, these wave functions pave the way to the exploration of the excited-state potential energy surface of large photoswitches in QMC.

\subsubsection{Synthetic photoswitches}

To enlarge the application area of dynamic molecules, many small organic photoswitches have been synthesized, e.g. azobenzene, spiropyran, diarylethene, thiophenefulgide, hemithioindigo, donor-acceptor Stenhouse adduct (DASA) and their derivatives. Each of these photoswitches has specific advantages and disadvantages, and is characterized by different wavelengths of maximum absorbance $\left(\lambda_{\max }\right)$ of the two or more isomers and by different structural and molecular properties upon photoswitching. For instance, azobenzenes can transform between two colored forms by illuminating with UV light at different wavelengths, and undergo significant structural changes upon photoswitching. On the other hand, conformational changes are small for diarylethenes since the isomerization only involves a bond breaking/forming process. Below, we focus on the DASA and spiropyran photoswitches which are investigated in this dissertation, due to the contrast in the appearance of the initial reactant and end product (colorless or colored) and the similarity in the complex switching reaction, which requires both ring opening/closure reaction and $E-Z$ stereoisomerization.

\section{Donor-acceptor Stenhouse adducts}

DASAs are new players among molecular photoswitches since they were discovered only in 2014 [30,31]. The conjugated system of DASAs is built by an amine/aniline donor and a diketo acceptor bridged by a triene system. In all solvents, DASAs exhibit negative photochromic properties in which shining visible light transforms the colored isomer to the colorless form as shown in Figure 1.2. When irradiation is stopped, the spontaneous backward switching occurs thermally except in polar sol- 
vents such as methanol where the photoswitching of DASA with an amine donor is irreversible. To enlarge the applicability area of DASAs in biological systems, the absorption maximum of the open isomer must be red-shifted by extending the conjugation of the acceptor and donor moieties, while preferably maintaining the planarity of the molecule. For instance, by replacing a Meldrum acid acceptor with a 1,3-dimethylbarbituric acid, the number of $\pi$ electrons increases by six, and consequently, $\lambda_{\max }$ red-shifts by about $25 \mathrm{~nm}$ [30]. In the donor part, lengthening the conjugation can be accomplished by substituting an amine group with an aniline. This has lead to the development of the so-called second-generation DASAs, which are red-shifted by as much as $110 \mathrm{~nm}[32,33]$.

a)<smiles>CCN(/C=C/C=C(\O)C=C1C(=O)N(C)C(=O)N(C)C1=O)CC</smiles>

b)<smiles>COc1ccc2c(c1)CCN2/C=C/C=C(\O)C=C1C(=O)N(C)C(=O)N(C)C1=O</smiles><smiles>CCN[C@@H]1C=CC(=O)C1c1c([O-])n(C)c(=O)n(C)c1=O</smiles><smiles>CCN[C@@H]1C=CC(=O)[C@H]1c1c([O-])n(C)c(=O)n(C)c1=O</smiles><smiles>COc1ccc2c(c1)CCN2C1C=CC(=O)[C@H]1C1C(=O)N(C)C(=O)N(C)C1=O</smiles>

Figure 1.2: The complete photoswitching reaction of a) an amine-based DASAs and b) an aniline-based DASAs with a 1,3-dimethylbarbituric acid acceptor.

The molecular photoswitching mechanism of open isomers of DASAs leading to the ring-closing event has been investigated using a combination of spectroscopic techniques and density functional theory calculations. It was inferred that the $Z-E$ isomerization of the middle double bond of the triene part occurs upon photoexcitation and that the subsequent reactions proceed thermally $[34,35]$. However, there is still no satisfying picture for the overall reaction leading primarily to a zwitterionic final product and a neutral diketo isomer for the amine-based and the aniline-based DASAs, respectively (see Figure 1.2). For a better design of DASA photoswitches, we unravel here all thermal steps of the complete reaction computationally and also establish the minimal computational ingredients for the evaluation of the excitedstate PES of the initial isomer.

Even though DASAs were only recently discovered, and the details of the molecular photoswitching mechanism are not fully understood yet, various promising applications have already emerged in material science and in biological systems [36]. 
For instance, a DASA molecule can constitute an amphiphile with a hydrophobic alkyl chains attached to the acceptor moiety and a hydrophilic tail connected to the donor part. The resulting amphiphile can self-assembly to form a micelle. In the presence of visible light, the DASA moiety of the amphiphile cyclizes yielding to structural disruption of the micelle [30]. This event can be utilized for drugdelivery applications where the drug is encapsulated inside the micelle structure in the dark [37].

\section{Spiropyrans}

Spiropyrans were first synthesized in the early twentieth century but their photochromism and reversibility of isomerization were reported later in 1954 [38]. As shown in Figure 1.3, spiropyran in its closed SP form consists of two conjugated systems bridged by a tetrahedral $\mathrm{C}_{\text {spiro }}$ atom, and consequently, its UV/Vis absorption spectrum is characterized by the presence of two peaks corresponding to the $\pi \rightarrow \pi^{*}$ electronic transitions in the indoline moiety $(272-296 \mathrm{~nm})$ and in the chromene moiety $(323-351 \mathrm{~nm})$ [39]. These two transitions belong to the ultraviolet wavelength region, so SP is colorless. Illuminating SP with UV light at $\lambda=365 \mathrm{~nm}$ (close to the $\lambda_{\max }$ of the chromene moiety) triggers the heterolitic $\mathrm{C}_{\text {spiro }}-\mathrm{O}$ bond cleavage [40] resulting in the cisoid MC species [41], which will eventually proceed thermally to the transoid $\mathrm{MC}$ isomer [42]. The backward-switching process (MC $\rightarrow \mathrm{SP}$ ) occurs instead thermally and can be accelerated with visible-light irradiation [3]. Interestingly, the photoswitching of SP can also be triggered with two photons in the near-infrared (NIR) region [43-47]. The two photons can transform SP to MC and further excite the $\mathrm{MC}$ isomer to obtain red fluorescence, which has been used for cellular imaging of cancer cells [48]. Moreover, since the two photons are in the NIR region reducing absorption-related photodamage in living systems, the two-photon laser scanning microscopy technique [49] can be employed to study biological processes beneath the tissue [50-53] by covalently attaching spiropyrans to the biomolecule of interest.

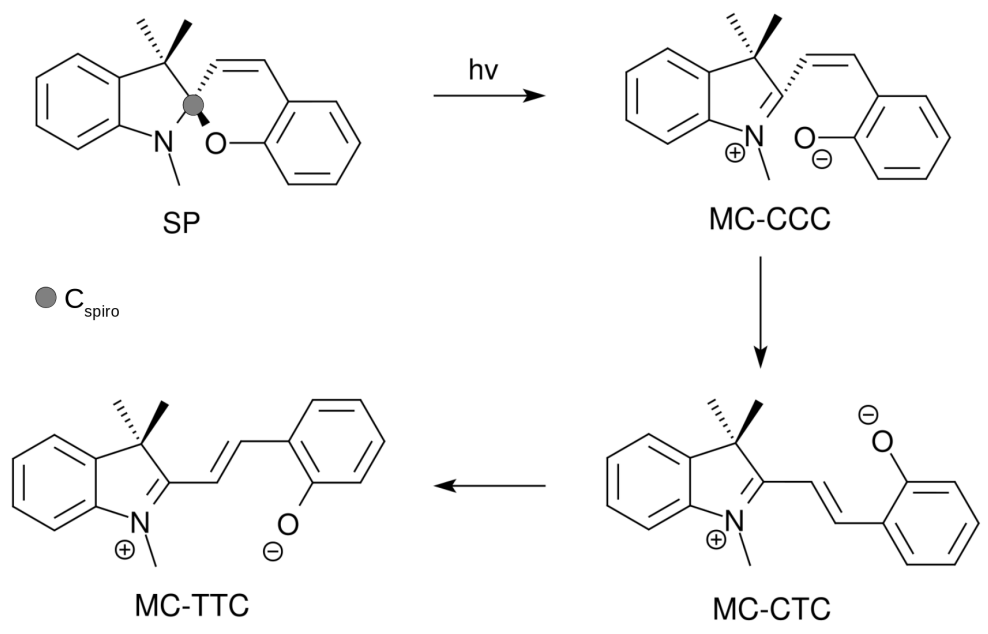

Figure 1.3: Photoisomerization of spiropyran from the closed SP to the open MC isomers. For simplicity, we only depict the zwitterionic resonance structure of MC. 


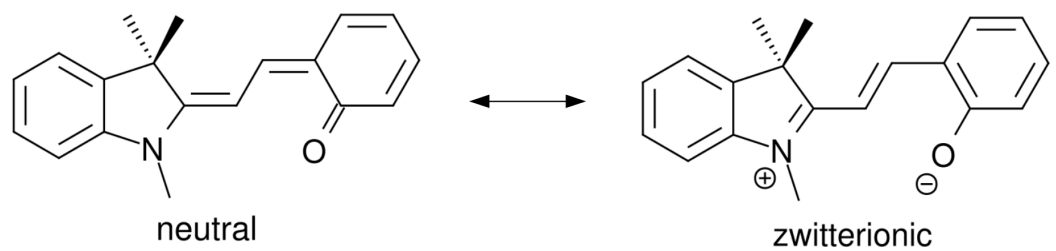

Figure 1.4: Two Lewis resonance structures of MC.

SP and MC isomers have very different optical and electronic properties which can be exploited for a variety of applications. First, the drastic change from a colorless (SP) to a colored (MC) state (due to the extended $\pi$ conjugation in MC) has been utilized for applications in molecular sensors [54]. Second, the location of the absorption maximum of $\mathrm{MC}$ can be tuned by adjusting the solvent polarity with the highest wavelength being obtained in nonpolar solvents [55]. This solvatochromism of MC has lead to the development of microcapillary systems for photodetection of solvent polarity $[56,57]$. Third, as opposed to the SP isomer, MC can be dissolved in a wide variety of solvents due to the presence of the two very different Lewis resonance structures depicted in Figure 1.4: the quinoidal neutral structure is favored in nonpolar media, while the zwitterionic structure is dominant in polar solvents. Interestingly, in polar solvents, MC is more stable than the hydrophobic SP form [3] to such an extent that photoswitching is started by illuminating $\mathrm{MC}$ with visible light to form SP and the MC isomer will be recovered thermally when the irradiation is stopped. Therefore, since the need for UV irradiation is avoided, this photoswitching behavior is particularly interesting for applications in biological systems where the photoswitch is attached covalently to a biomolecule support and exposed mainly to the water-based solvent.

One of the earliest application of photoswitching of SP and MC in biopolymers was in the photocontrol of polypeptide formation [58]. Multiple photoswitches in the MC form were connected covalently to a short polypeptide made of glutamic acid monomers. The photoswitching of MC upon visible-light irradiation was found to induce a secondary-structure change of the polypeptide from a random coil to an $\alpha$ helix through a mechanism which has been a matter of some debate $[59,60]$. Other more applicative examples include the photocontrol of transport through biopolymers [61], of DNA hybridizations [62] and of enzymatic activities [63]. Recently, our experimental collaborators have covalently connected a single SP molecule to a protein and found that the photoswitching of SP induces allosterically ligand release at a distinct binding pocket [64]. Interestingly, unlike in the commonly investigated hybrid biopolymers, the photoswitch in our study exists in the SP form which indicates that it interacts more frequently with the protein than with water. Here, we shed light on the ligand-release mechanism with extensive molecular dynamics simulations of the hybrid protein-photoswitch ( $\mathrm{SP}$ and $\mathrm{MC}$ ) systems. These simulations required the construction of the force field for the SP and MC isomers via fitting to quantum mechanical calculations. For this problem, excited-state dynamical studies are not necessary in explaining the experimental measurements which were performed at equilibrium conditions. 


\subsection{This dissertation}

We present in Chapter 3 our novel class of multideterminant Jastrow-Slater wave functions for the efficient and accurate treatment of excited-states PES in quantum Monte Carlo calculations. In our wave functions, we include excitations from multiple sets of localized orbitals corresponding to the different dominant Lewis resonance structures of the molecule. Instead of employing all determinants built from the active space, we perform a smart truncation by correlating orbitals according to the concept of orbital domains of local coupled-cluster methods and construct single, double and higher excitations from one or multiple orbital domains. The excitations are collected in classes which are ordered in importance and included systematically in the wave-function expansion.

The performance of these local wave functions is assessed by calculating the vertical excitation energies and optimal excited-state geometries of retinal models whose lowest bright excited state has a strong charge-transfer character. We find that our multi-resonance wave functions are very compact since they comprise only the doubly-occupied reference(s), the important single excitations and the bondingantibonding double excitations. Importantly, in contrast to the traditional truncation procedure based on the threshold on the MCSCF expansion coefficients (which does not guarantee that the same determinants are taken at different geometries), our novel wave functions contain the same set of determinants that can be flexibly and accurately applied to very different parts of the excited-state PES. We also demonstrate that, for larger retinal models, the number of determinants does not grow exponentially and that the size of the orbital domains saturates since faraway orbitals become weakly correlated. Therefore, our multi-resonance wave functions are very promising in treating excited states of large photo-responsive molecules such as the retinal, DASA and spiropyran photoswitches discussed above.

Next, motivated by the lack of knowledge of a detailed photoswitching mechanism of DASA molecules, we study the complete thermal reaction of the molecular isomerization using density functional theory and an implicit solvent model in Chapter 4. Our computational study provides an explanation for several available experimental observations. For the amine-based DASAs, we are able to explain the irreversibility/reversibility of photoswitching and the longer/shorter irradiation time needed for the full conversion towards the closed isomer in methanol/toluene. For the aniline-based DASAs, our calculations support the experimental finding that the isomerization towards the neutral diketo final product becomes more important than in the amine-based DASAs. Our mechanistic thermal pathways can therefore be used to predict the behavior of other DASA derivatives. We also present a preliminary study of the excited-state relaxation of DASA with multiconfigurational wave function methods. We find that these approaches reveal severe shortcomings and that the excited-state PES of this system should therefore be calculated by including a balanced description of both static and dynamical electron correlations. This can be accomplished with either the CASPT2 method or QMC in combination with our novel multi-resonance wave functions in future studies. 
Finally, we investigate the functional properties of a photoswitch-protein hybrid system in Chapter 5. Our experimental collaborators have covalently coupled a single spiropyran unit to the surface-accessible cysteine residue of the human serum albumin protein and found that the photo-induced SP to MC transformation is correlated to ligand release at a near but distinct binding pocket. The human serum albumin protein is chosen because it is one of the most abundant protein in plasma and, despite its simple monomeric form, can bind over 120 ligands and seven fatty acids, therefore functioning as a transporting protein. To understand the molecular mechanism of ligand release, we first investigate the possibility of interaction between the protein, photoswitch and ligand using molecular docking techniques and evaluate the stability of the resulting noncovalent interactions with molecular dynamics simulations. Our simulations confirm the allosteric nature of the interactions between the subdomain where the photoswitch is attached and the pocket where the ligand sits. We identify a key hydrogen bond that is perturbed upon attaching and further switching the SP and suggest that the increased fluctuation of the backbone upon photoswitching correlates to the experimentally observed ligand release. 


\section{Bibliography}

[1] W. Szymański, J. M. Beierle, H. A. Kistemaker, W. A. Velema, and B. L. Feringa, Chem. Rev. 113, 6114 (2013).

[2] R. Klajn, J. F. Stoddart, and B. A. Grzybowski, Chem. Soc. Rev. 39, 2203 (2010).

[3] R. Klajn, Chem. Soc. Rev. 43, 148 (2014).

[4] A. Grinthal and J. Aizenberg, Chem. Soc. Rev. 42, 7072 (2013).

[5] M. Irie, Chem. Rev. 100, 1685 (2000).

[6] M. Yamada, M. Kondo, J.-i. Mamiya, Y. Yu, M. Kinoshita, C. J. Barrett, and T. Ikeda, Angew. Chem. Int. Ed. 47, 4986 (2008).

[7] C. A. Ullrich, Time-dependent density-functional theory: concepts and applications (OUP Oxford, 2011).

[8] M. E. Casida and M. Huix-Rotllant, Annu. Rev. Phys. Chem. 63, 287 (2012).

[9] R. Send, M. Kühn, and F. Furche, J. Chem. Theory Comput. 7, 2376 (2011).

[10] R. Guareschi and C. Filippi, J. Chem. Theory Comput. 9, 5513 (2013).

[11] F. Santoro and D. Jacquemin, WIREs: Comput. Mol. Sci. 6, 460 (2016).

[12] B. O. Roos, P. R. Taylor, and P. E. Siegbahn, Chem. Phys. 48, 157 (1980).

[13] A. Lüchow, WIREs: Comput. Mol. Sci. 1, 388 (2011).

[14] C. Filippi, R. Assaraf, and S. Moroni, J. Chem. Phys. 144, 194105 (2016).

[15] M. A. Thompson, J. Phys. Chem. 100, 14492 (1996).

[16] C. Curutchet, A. Muñoz-Losa, S. Monti, J. Kongsted, G. D. Scholes, and B. Mennucci, J. Chem. Theory Comput. 5, 1838 (2009).

[17] J. M. Olsen, K. Aidas, and J. Kongsted, J. Chem. Theory Comput. 6, 3721 (2010).

[18] L. V. Slipchenko, J. Phys. Chem. A 114, 8824 (2010). 
[19] R. Guareschi, H. Zulfikri, C. Daday, F. M. Floris, C. Amovilli, B. Mennucci, and C. Filippi, J. Chem. Theory Comput. 12, 1674 (2016).

[20] S. Gozem, H. L. Luk, I. Schapiro, and M. Olivucci, Chem. Rev. 117, 13502 (2017).

[21] R. Schoenlein, L. Peteanu, R. Mathies, and C. Shank, Science 254, 412 (1991).

[22] M. Garavelli, F. Negri, and M. Olivucci, J. Am. Chem. Soc. 121, 1023 (1999).

[23] V. Buß, K. Kolster, F. Terstegen, and R. Vahrenhorst, Angew. Chem. Int. Ed. 37, 1893 (1998).

[24] D. Polli, P. Altoè, O. Weingart, K. M. Spillane, C. Manzoni, D. Brida, G. Tomasello, G. Orlandi, P. Kukura, R. A. Mathies, M. Garavelli, and G. Cerullo, Nature 467, 440 (2010).

[25] D. Polli, O. Weingart, D. Brida, E. Poli, M. Maiuri, K. M. Spillane, A. Bottoni, P. Kukura, R. A. Mathies, G. Cerullo, and M. Garavelli, Angew. Chem. Int. Ed. 53, 2504 (2014).

[26] O. Valsson and C. Filippi, J. Chem. Theory Comput. 6, 1275 (2010).

[27] S. Gozem, F. Melaccio, R. Lindh, A. I. Krylov, A. A. Granovsky, C. Angeli, and M. Olivucci, J. Chem. Theory Comput. 9, 4495 (2013).

[28] J. W. Park and T. Shiozaki, ArXiv e-prints: 1802.00096 (2018).

[29] N. Coughlan, B. Adamson, L. Gamon, K. Catani, and E. Bieske, Phys. Chem. Chem. Phys. 17, 22623 (2015).

[30] S. Helmy, F. A. Leibfarth, S. Oh, J. E. Poelma, C. J. Hawker, and J. Read de Alaniz, J. Am. Chem. Soc. 136, 8169 (2014).

[31] S. Helmy, S. Oh, F. A. Leibfarth, C. J. Hawker, and J. Read de Alaniz, J. Org. Chem. 79, 11316 (2014).

[32] J. R. Hemmer, S. O. Poelma, N. Treat, Z. A. Page, N. D. Dolinski, Y. J. Diaz, W. Tomlinson, K. D. Clark, J. P. Hooper, C. Hawker, and J. Read de Alaniz, J. Am. Chem. Soc. 138, 13960 (2016).

[33] N. Mallo, P. T. Brown, H. Iranmanesh, T. S. MacDonald, M. J. Teusner, J. B. Harper, G. E. Ball, and J. E. Beves, Chem. Commun. 52, 13576 (2016).

[34] M. M. Lerch, S. J. Wezenberg, W. Szymański, and B. L. Feringa, J. Am. Chem. Soc. 138, 6344 (2016).

[35] M. Di Donato, M. M. Lerch, A. Lapini, A. D. Laurent, A. Iagatti, L. Bussotti, S. P. Ihrig, M. Medved', D. Jacquemin, W. Szymański, W. J. Buma, P. Foggi, and B. L. Feringa, J. Am. Chem. Soc. 139, 15596 (2017). 
[36] M. M. Lerch, W. Szymański, and B. L. Feringa, Chem. Soc. Rev. 47, 1910 (2018).

[37] S. O. Poelma, S. S. Oh, S. Helmy, A. S. Knight, G. L. Burnett, H. T. Soh, C. J. Hawker, and J. R. de Alaniz, Chem. Commun. 52, 10525 (2016).

[38] Y. Hirshberg and E. Fischer, J. Chem. Soc. 297 (1954).

[39] N. W. Tyer Jr and R. S. Becker, J. Am. Chem. Soc. 92, 1289 (1970).

[40] H. Görner, Phys. Chem. Chem. Phys. 3, 416 (2001).

[41] S. Krysanov and M. Alfimov, Chem. Phys. Lett. 91, 77 (1982).

[42] C. Lenoble and R. S. Becker, J. Phys. Chem. 90, 62 (1986).

[43] D. A. Parthenopoulos and P. M. Rentzepis, Science 245, 843 (1989).

[44] A. Dvornikov, J. Malkin, and P. Rentzepis, J. Phys. Chem. 98, 6746 (1994).

[45] S. O. Konorov and A. M. Zheltikov, Opt. Express 11, 2440 (2003).

[46] M. C. George, A. Mohraz, M. Piech, N. S. Bell, J. A. Lewis, and P. V. Braun, Adv. Mater. 21, 66 (2009).

[47] O. Ivashenko, J. T. van Herpt, B. L. Feringa, P. Rudolf, and W. R. Browne, Langmuir 29, 4290 (2013).

[48] M.-Q. Zhu, G.-F. Zhang, C. Li, M. P. Aldred, E. Chang, R. A. Drezek, and A. D. Li, J. Am. Chem. Soc. 133, 365 (2010).

[49] W. Denk, J. H. Strickler, and W. W. Webb, Science 248, 73 (1990).

[50] M. Drobizhev, N. S. Makarov, S. E. Tillo, T. E. Hughes, and A. Rebane, Nat. Methods 8, 393 (2011).

[51] W. R. Zipfel, R. M. Williams, and W. W. Webb, Nat. Biotech. 21, 1369 (2003).

[52] P. S. Tsai, B. Friedman, A. I. Ifarraguerri, B. D. Thompson, V. Lev-Ram, C. B. Schaffer, Q. Xiong, R. Y. Tsien, J. A. Squier, and D. Kleinfeld, Neuron 39, 27 (2003).

[53] J. Herz, V. Siffrin, A. E. Hauser, A. U. Brandt, T. Leuenberger, H. Radbruch, F. Zipp, and R. A. Niesner, Biophys. J. 98, 715 (2010).

[54] B. Mason, M. Whittaker, J. Hemmer, S. Arora, A. Harper, S. Alnemrat, A. McEachen, S. Helmy, J. Read de Alaniz, and J. Hooper, Appl. Phys. Lett. 108, 041906 (2016).

[55] R. Rosario, D. Gust, M. Hayes, J. Springer, and A. A. Garcia, Langmuir 19, 8801 (2003). 
[56] L. Florea, A. Hennart, D. Diamond, and F. Benito-Lopez, Sens. Actuators, B 175, 92 (2012).

[57] L. Florea, A. McKeon, D. Diamond, and F. Benito-Lopez, Langmuir 29, 2790 (2013).

[58] F. Ciardelli, D. Fabbri, O. Pieroni, and A. Fissi, J. Am. Chem. Soc. 111, 3470 (1989).

[59] N. Angelini, B. Corrias, A. Fissi, O. Pieroni, and F. Lenci, Biophys. J. 74, 2601 (1998).

[60] R. Pachter, T. M. Cooper, L. Natarajan, K. A. Obermeier, R. L. Crane, and W. W. Adams, Biopolymers 32, 1129 (1992).

[61] I. Karube, Y. Ishimori, and S. Suzuki, Anal. Biochem. 86, 100 (1978).

[62] H. Asanuma, K. Shirasuka, T. Yoshida, T. Takarada, X. Liang, and M. Komiyama, Chem. Lett. 30, 108 (2001).

[63] T. Sakata, Y. Yan, and G. Marriott, Proc. Nat. Acad. Sci. 102, 4759 (2005).

[64] R. M. Putri, Optical Control over Monomeric and Multimeric Protein Hybrids (University of Twente, 2017). 


\section{Chapter 2}

\section{Theoretical methods}

In this dissertation, we use different theoretical approaches ranging from electronic structure methods for relatively small systems to atomistic simulations. In this chapter, we briefly describe the theoretical background behind our methodological developments and simulations. We start with the quantum mechanical methods for small molecular systems, which are primarily used to describe the optical properties and the thermochemistry of isomerization reactions, where explicit consideration of electron is mandatory. For a biosystem of more than 10000 atoms, we resort to a classical mechanics description to understand the dynamical properties of a photo-responsive engineered biomolecule.

\subsection{Quantum mechanical calculations}

In the electronic structure simulations throughout this work, we make use of the Born-Oppenheimer approximation [1] and omit relativistic effects since the heaviest atom we consider is oxygen. The resulting electronic Hamiltonian takes therefore the following form in atomic units $(\hbar=m=e=1)$ :

$$
\mathcal{H}=-\frac{1}{2} \sum_{i=1}^{N} \nabla_{i}^{2}+\sum_{i=1}^{N} v_{\mathrm{ext}}\left(\mathbf{r}_{i}\right)+\sum_{i<j}^{N} \frac{1}{\left|\mathbf{r}_{i}-\mathbf{r}_{j}\right|}
$$

The first term is the kinetic energy operator of the electrons, the second term the electron-ion Coulomb potential, and the last term the electron-electron interaction. All difficulties in electronic structure calculations arise from the fact that this last term is not separable in single-electron operators like the first two terms.

We briefly discuss below wave-function based and effective one-electron theories commonly used to calculate the thermochemistry of chemical reactions.

\subsubsection{Single-determinant wave function}

The starting point to understand how one can treat the many-electron interacting problem is in fact the antisymmetric wave function describing a non-interacting sys- 
tem, namely, a so-called Slater determinant (SD):

$$
\Psi_{\mathrm{SD}}\left(\mathbf{x}_{1}, \mathbf{x}_{2}, \cdots, \mathbf{x}_{N}\right)=\frac{1}{\sqrt{N !}}\left|\begin{array}{cccc}
\Phi_{1}\left(\mathbf{x}_{1}\right) & \Phi_{1}\left(\mathbf{x}_{2}\right) & \cdots & \Phi_{1}\left(\mathbf{x}_{N}\right) \\
\Phi_{2}\left(\mathbf{x}_{1}\right) & \Phi_{2}\left(\mathbf{x}_{2}\right) & \cdots & \Phi_{2}\left(\mathbf{x}_{N}\right) \\
\vdots & \vdots & \vdots & \vdots \\
\Phi_{N}\left(\mathbf{x}_{1}\right) & \Phi_{N}\left(\mathbf{x}_{2}\right) & \cdots & \Phi_{N}\left(\mathbf{x}_{N}\right)
\end{array}\right|
$$

where $\{\Phi\}$ are single-particle orbitals (spin-orbitals) depending on the space-spin electron coordinates, $\mathbf{x}=(\mathbf{r}, \sigma)$. The spin-orbitals are usually written as products of spatial and spin functions, i.e. $\Phi_{i}(\mathbf{x})=\phi_{i}(\mathbf{r}) \chi_{s_{i}}(\sigma)$. With this wave function, the electrons are indistinguishable, and two electrons cannot occupy the same spacespin coordinate as appropriate for a fermionic system [2]. The spatial component of the spin-orbital can be expressed as a linear combination of atomic orbitals (LCAO) centered on the nuclear positions:

$$
\phi_{i}(\mathbf{r})=\sum_{\mu}^{\text {nuclei }} \sum_{j} a_{j i}^{\mu} \eta_{j \mu}\left(\mathbf{r}-\mathbf{r}_{\mu}\right),
$$

where $\mathbf{r}_{\mu}$ denotes the position of a nucleus. Gaussian atomic basis functions

$$
\eta(\mathbf{r})=x^{m} y^{n} z^{k} \exp \left(-\alpha r^{2}\right)
$$

are commonly used in quantum chemical calculations since this choice allows one to compute all integrals encountered in the computation of observables analytically.

If one requires that this is the best non-interacting wave function and minimizes the energy while keeping the orthonormality of the molecular spatial orbitals, one finds that the orbitals must satisfy the so-called Hartree-Fock (HF) equations:

$$
\hat{f}(i)\left|\phi_{i}\right\rangle=\sum_{j=1}^{N} \epsilon_{i j}\left|\phi_{j}\right\rangle,
$$

where the sum runs over the occupied orbitals and $\hat{f}$ is the single-particle Fock operator,

$$
\hat{f}(i)=-\frac{1}{2} \nabla_{i}^{2}+v_{\text {ext }}(i)+v^{\mathrm{HF}}(i) .
$$

The Hartree-Fock potential $v^{\mathrm{HF}}$ is the summation over the Coulombic and exchange terms:

$$
\begin{aligned}
\left(v^{\mathrm{HF}} \phi_{i}\right)(\mathbf{r}) & =\left(v_{\text {Coulomb }} \phi_{i}\right)(\mathbf{r})+\left(v_{\mathrm{x}}^{\mathrm{HF}} \phi_{i}\right)(\mathbf{r}) \\
\left(v_{\text {Coulomb }} \phi_{i}\right)(\mathbf{r}) & =\left[\sum_{j=1}^{N} \int \frac{\left|\phi_{j}\left(\mathbf{r}^{\prime}\right)\right|^{2}}{\left|\mathbf{r}-\mathbf{r}^{\prime}\right|} d \mathbf{r}^{\prime}\right] \phi_{i}(\mathbf{r}) \\
\left(v_{\mathrm{x}}^{\mathrm{HF}} \phi_{i}\right)(\mathbf{r}) & =-\sum_{j=1}^{N} \delta_{s_{i}, s_{j}}\left[\int \frac{\phi_{j}^{*}\left(\mathbf{r}^{\prime}\right) \phi_{i}\left(\mathbf{r}^{\prime}\right)}{\left|\mathbf{r}-\mathbf{r}^{\prime}\right|} d \mathbf{r}^{\prime}\right] \phi_{j}(\mathbf{r}) .
\end{aligned}
$$


The Coulombic potential (also called Hartree potential) accounts for the electrostatic interaction between an electron in $\phi_{i}(\mathbf{r})$ with the charge distribution of system. The $j=i$ contribution to the exchange potential cancels the self-interaction term in the Hartree potential due to the interaction of the electron with itself, while the $j \neq i$ contributions for orbitals with the same spin quantum number arise from the antisymmetry of the wave function, thus have no equivalent in a classical description. To solve these equations, one uses an initial set of LCAO coefficients to construct the corresponding HF potential and then evaluates the HF equations to obtain a new set of molecular orbitals. One iterates until there are no changes in the LCAO coefficients and, at convergence, finds the self-consistent solutions [3].

\subsubsection{Correlation methods}

The HF wave function is the best non-interacting solution to an interacting problem, and the resulting HF energy is of course higher than the exact energy $E$, with the difference being called the correlation energy:

$$
E_{\mathrm{corr}}=E-E_{\mathrm{HF}} \text {. }
$$

The simplest approach to account for (part of) the correlation is to expand the wave function in term of multiple Slater determinants yielding a so-called configuration interaction $(\mathrm{CI})$ wave function:

$$
\Psi=c_{0} \Psi_{\mathrm{HF}}+c_{1} \Psi_{1}+c_{2} \Psi_{2}+\ldots
$$

where the determinants $\left\{\Psi_{n}\right\}$ differ from the HF determinant by one or more orbitals. For a given basis set (or equivalently a given set of orbitals), one can in principle expand over all $N$-electron excitations in the complete orbital space recovering the full configuration interaction (FCI) wave function and, correspondingly, the best energy for the given basis set. Unfortunately, the FCI limit is only feasible for very small molecules, and approximations are generally introduced when going to larger systems.

In the case of frontier orbitals being very close in energy, excitations to these orbitals may acquire significant contribution (even similar weight to the HF determinant) in the total wave function. This is typically the case for conjugated organic molecules, transition-metal and rare-earth-metal complexes. A description in terms of a single determinant is then inadequate, and other important excitations are required in the expansion. This type of correlation is called static electron correlation, and its treatment requires a so-called multiconfiguration self-consistent-field (MCSCF) wave function, where both the expansion coefficients and the occupied and unoccupied molecular orbitals are optimized. In this dissertation, we often employ a special case of MCSCF expansions called complete-active-space self-consistent field (CASSCF), where one employs a full CI expansion with $n$ electrons distributed over a set of $m$ active orbitals in a so-called $\operatorname{CAS}(n, m)$ wave function [4,5]. The occupied and unoccupied orbitals outside the active space are termed inactive and virtual 
orbitals, respectively, and their role remains very important. By correlating these orbitals, one also accounts for the missing dynamical electron correlation to the total energy.

If one deals with multiple electronic states, it is convenient to expand the excitedstate wave functions using the same set of determinants as in the ground state to enable maintaining orthogonality between states of the same symmetry via the CI coefficients. The HF determinant only minorly contributes to the excited-state wave function, and usually, for the lowest singlet excited state, the transition from the highest occupied molecular orbital (HOMO) to the lowest unoccupied molecular orbital (LUMO) dominates. It is important that the states are treated on the same footing and that the set of orbitals is a good compromise for all states of interest. To this aim, one optimizes the orbitals in a state-averaged approach by minimizing

$$
E_{\mathrm{SA}}=\sum_{I} w_{I} \frac{\left\langle\Psi_{I}|\mathcal{H}| \Psi_{I}\right\rangle}{\left\langle\Psi_{I} \mid \Psi_{I}\right\rangle}
$$

where $\sum_{I} w_{I}=1$, while preserving orthogonality between the states thanks to the CI coefficients. Optimizing a state-average energy instead of targeting directly the excited-state energy also avoids instabilities and root-flipping problems which will occur if the gap between two states is small. Since the minimized quantity is the state-averaged energy, the resulting orbitals are neither optimal for the ground state nor for the excited states. Nevertheless, the energy gap between the states is often reasonable, and the agreement to experimental excitation energies can be further improved by including dynamical correlation. We note that, typically, one employs the same weights on all states as impartial weight assignment will lead to the overstabilization of the state with larger weight and, consequently, alter the vertical excitation energy.

The missing electron correlation in MCSCF wave functions can be partially included by using the Rayleigh-Schrödinger perturbation theory, where the total Hamiltonian is split into a zero-order Hamiltonian, $\mathcal{H}^{(0)}$, and a perturbing operator $\mathcal{V}$ :

$$
\mathcal{H}=\mathcal{H}^{(0)}+\mathcal{V}
$$

When considering the HF wave function as the zero-order wave function, one obtains the n-th order Møller-Plesset perturbation theory (MPn) [6]. If CASSCF is the zero-order wave function, the resulting method is called the complete-active-space with n-th order perturbation theory (CASPTn) $[7,8]$ where usually the perturbation is considered until the second order. In CASPTn theory, there is no unique definition of the zero-order Hamiltonian, and one can choose different formulations as long as they result in the same zero-order CASSCF wave function. One of the most commonly employed formulations of the zero-order one-body Hamiltonian in CASPTn calculations is based on an effective one-body Fock operator and requires a so-called ionization potential-electronic affinity (IPEA) shift [9] of 0.25 a.u., which is a semiempirical parameter that compensates a discrepancy between orbital energies when exciting from/into the active space. The introduction of the IPEA shift significantly changes the CASPT2 energy, in particular for the excited states: for instance, without 
the shift, the vertical excitation energies are found to be systematically too low [10], and thus, one has to be aware of the type of zero-order Hamiltonian used before making any comparison. The CASPT 2 method has been used widely to calculate excited-state properties, and it was shown that, in organic molecules, the relative CASPT2 energies of different states vary less with the use of small or large active spaces than the CASSCF counterparts, which are instead significantly active-space dependent [11]. Therefore, CASPT2 is capable of correcting some of the shortcomings of CASSCF and accounts for part of the missing static and dynamical correlation. However, the performance of CASPT2 with variation in the size of active space in calculating the potential energy surface (PES) is less known as typically the minimal active space is used to afford the costly numerical energy-gradient calculations. Finally, we note that, while the third-order theory [12] is rarely employed due to the increase of computational cost, its use appears to improve the quality of the CASPT2 excitation energies and reduce the sensitivity of the approach to the choice of the zero-order Hamiltonian [13].

\subsubsection{Quantum Monte Carlo methods}

In dealing with large systems, one can resort to an alternative class of techniques, that is, quantum Monte Carlo (QMC) methods which provide a stochastic way of approximately solving the Schrödinger equation [14]. Since one computes the integral via Monte Carlo sampling, one has great freedom in the choice of the form of the wave function (i.e. inclusion of electron-electron correlation terms, use of non-orthogonal molecular orbitals etc.). Moreover, the cost of QMC calculations is rather favorable, scaling as $\mathrm{N}^{4}$ with the number of electrons, and the stochastic nature of the method allows the calculations to be efficiently parallelized in modern supercomputers.

The simplest variant of QMC is the variational Monte Carlo (VMC) method, which is simply a way to compute the expectation value of an operator on a given wave function. For a trial wave function $\Psi_{\mathrm{T}}$, the expectation value of electronic energy $E_{V}$ is given by

$$
E_{V}=\frac{\int \Psi_{\mathrm{T}}^{*}(\mathbf{R}) \mathcal{H} \Psi_{\mathrm{T}}(\mathbf{R}) d \mathbf{R}}{\int \Psi_{\mathrm{T}}^{*}(\mathbf{R}) \Psi_{\mathrm{T}}(\mathbf{R}) \mathrm{d} \mathbf{R}}
$$

where $\mathcal{H}$ is the electronic Hamiltonian, and $\mathbf{R}$ denotes the $3 \mathrm{~N}$ electron spatial coordinates. Factorizing $\Psi_{\mathrm{T}}(\mathbf{R})$ both in the nominator and the denominator, one arrives at

$$
\begin{aligned}
E_{V} & =\int \frac{\left|\Psi_{\mathrm{T}}(\mathbf{R})\right|^{2}}{\int\left|\Psi_{\mathrm{T}}\left(\mathbf{R}^{\prime}\right)\right|^{2} \mathrm{~d} \mathbf{R}^{\prime}} \frac{\mathcal{H} \Psi_{\mathrm{T}}(\mathbf{R})}{\Psi_{\mathrm{T}}(\mathbf{R})} \mathrm{d} \mathbf{R} \\
& =\int \rho(\mathbf{R}) \frac{\mathcal{H} \Psi_{\mathrm{T}}(\mathbf{R})}{\Psi_{\mathrm{T}}(\mathbf{R})} \mathrm{d} \mathbf{R} \\
& =\int \rho(\mathbf{R}) E_{L}(\mathbf{R}) \mathrm{d} \mathbf{R}
\end{aligned}
$$

Here, $\rho(\mathbf{R})$ is the normalized square of the wave function, which is positive and integrates to one, and can therefore be interpreted as probability density function, while 
$E_{L}(\mathbf{R})$ is the local energy of the system. Like in classical Monte Carlo, this integral can be computed by sampling a set of configurations $\left\{\mathbf{R}_{m}\right\}$ distributed according to the $\rho(\mathbf{R})$. The expectation value then becomes

$$
E_{V} \approx \frac{1}{M} \sum_{m=1}^{M} E_{L}\left(\mathbf{R}_{m}\right) .
$$

Given the high-dimensionality $(3 \mathrm{~N})$ of the integral and the fact that the normalization of the square of the trial wave function is difficult to compute for the molecular systems studied here, we employ the Metropolis algorithm [15] to obtain a sequence of electronic coordinates which is asymptotically distributed according to $\rho(\mathbf{R})$. More specifically, we use the accelerated Metropolis method [16] appropriately modified to work with non-local pseudopotentials.

The trial wave function used in our QMC calculations is of the so-called JastrowSlater form, which is the product of a Jastrow correlation factor, $\mathcal{J}$, which depends on the interparticle distances, and a linear combination of Slater determinants. In a spin-assigned form, it is written as

$$
\Psi\left(\mathbf{r}_{1}, \ldots, \mathbf{r}_{N}\right)=\mathcal{J}\left(\mathbf{r}_{1}, \ldots, \mathbf{r}_{N}\right) \sum_{k} d_{k} D_{k}^{\uparrow}\left(\mathbf{r}_{1}, \ldots, \mathbf{r}_{N_{\uparrow}}\right) D_{k}^{\downarrow}\left(\mathbf{r}_{N_{\uparrow}+1}, \ldots, \mathbf{r}_{N}\right),
$$

where $D_{k}^{\uparrow}$ and $D_{k}^{\downarrow}$ are Slater determinants of single-particle orbitals for the up- and down-spin electrons, respectively. The expansion can be constructed with either delocalized or localized orbitals or a combination of both since the flexibility of QMC also allows us to work with an over-complete set of orbitals. The Jastrow-Slater wave functions describe both types of electron correlations: dynamical correlation is partially accounted for by the Jastrow factor, while static correlation is covered by the multiple Slater determinants, whose expansion will be more compact than a CI wave function thanks to the presence of the Jastrow factor. We note that the presence of the Jastrow factor renders the analytical computation of the integrals of expectation values impossible.

In our calculations, we use the following simple form of Jastrow factor only including electron-nucleus and electron-electron correlations:

$$
\mathcal{J}\left(\mathbf{r}_{1}, \ldots, \mathbf{r}_{N}\right)=\prod_{\alpha, i} \exp \left\{A\left(r_{i \alpha}\right)\right\} \times \prod_{i<j} \exp \left\{B\left(r_{i j}\right)\right\}
$$

where $A\left(r_{i \alpha}\right)$ depends on the electron-nucleus distances, $r_{i \alpha}$, and $B\left(r_{i j}\right)$ on the electron-electron distances, $r_{i j}$. Both functions are fifth-order polynomials, and the arguments are scaled variables as $\bar{r}=\left(1-e^{\kappa r}\right) / \kappa$ to ensure a regular behavior of the Jastrow factor at large interparticle distances. Different atom types have different coefficients of the electron-nucleus polynomials. The function $B\left(r_{i j}\right)$ includes an additional term $b_{1} \bar{r}_{i j} /\left(1+b_{2} \bar{r}_{i j}\right)$ with $b_{1}=1 / 2$ and $1 / 4$ for opposite- and like-spin electrons, respectively, which is linear at short distances and ensures that the wave function satisfies the Kato's cusp conditions, i.e. an appropriate discontinuity of the derivatives of the wave function at the coalescence points. 
Despite the compactness of the trial wave functions, one should ensure that the selected determinants represent the important physics of the system. The selection is usually performed based on chemical intuition as well as on a threshold on the expansion coefficients in the MCSCF wave functions. The latter procedure does not guarantee that the same set of determinants is included for different parts of the potential energy surface. This shortcoming has prompted us to explore a better scheme to truncate the determinantal expansion based on a local-orbital representation.

After establishing the component of the wave functions, all parameters should be reoptimized since the initial determinantal expansion coefficients and orbitals from a HF or MCSCF calculation are obtained in the absence of the Jastrow factor. For the ground-state problem, this is achieved by using the linear optimization method within energy minimization [17], which is an extension to arbitrary wave functions in QMC methods of the so-called super-CI approach of wave function optimization in MCSCF. Optimizing parameters in the multiple-state case is somewhat more complicated and is achieved by minimizing the state-averaged energy (Eq. 2.11), where different states share the same Jastrow factor and orbitals but are characterized by different sets of CI coefficients and are orthogonal [18].

It is possible to improve on a given trial wave function by projecting stochastically a better approximation of the state of interest. In diffusion Monte Carlo (DMC), one employs the projection operator $\exp \left[-\tau\left(\mathcal{H}-E_{\mathrm{T}}\right)\right]$ and, by repeatedly applying this operator to an initial trial wave function, obtains the sequence of wave functions:

$$
\Psi^{(n)}=e^{-\tau\left(\mathcal{H}-E_{\mathrm{T}}\right)} \Psi^{(n-1)} .
$$

If we expand the initial wave function $\Psi^{(0)}$ on the eigenstates $\Psi_{i}$ with energies $E_{i}$ of $\mathcal{H}$, we obtain

$$
\Psi^{(n)}=\sum_{i} \Psi_{i}\left\langle\Psi^{(0)} \mid \Psi_{i}\right\rangle e^{-n \tau\left(E_{i}-E_{\mathrm{T}}\right)},
$$

and, since the excited-state coefficients decay more quickly, we have for large $n$

$$
\lim _{n \rightarrow \infty} \Psi^{(n)}=\Psi_{0}\left\langle\Psi^{(0)} \mid \Psi_{0}\right\rangle e^{-n \tau\left(E_{0}-E_{\mathrm{T}}\right)} .
$$

Hence, the projection results in principle in the ground-state $\Psi_{0}$ of the Hamiltonian if we adjust the trial energy $E_{\mathrm{T}} \approx E_{0}$ to keep the overall normalization of $\Psi^{(n)}$ fixed.

To understand how to perform the projection in practice, let us rewrite Eq. 2.17 in integral form as:

$$
\Psi^{(n)}\left(\mathbf{R}^{\prime}, t+\tau\right)=\int \mathrm{d} \mathbf{R} G\left(\mathbf{R}^{\prime}, \mathbf{R}, \tau\right) \Psi^{(n-1)}(\mathbf{R}, t),
$$

where the imaginary-time Green's function is defined as:

$$
G\left(\mathbf{R}^{\prime}, \mathbf{R}, \tau\right)=\left\langle\mathbf{R}^{\prime}\left|e^{-\tau\left(\mathcal{H}-E_{\mathrm{T}}\right)}\right| \mathbf{R}\right\rangle .
$$

If we can sample the trial wave function and the Green's function in Eq. 2.20, we can compute this integral stochastically by Monte Carlo integration. Unfortunately, one 
encounters two complications: 1) the analytical expression of the Green function is not known and is approximated for small time steps, so one needs to perform multiple DMC calculations and extrapolates as $\tau \rightarrow 0 ; 2$ ) the electrons are fermions and we cannot interpret the antisymmetric wave function as a probability distribution. To address this problem, one rewrites the above integral equation in terms of the product $f^{(n)}(\mathbf{R}, t)=\Psi_{\mathrm{T}}(\mathbf{R}) \Psi^{(n)}(\mathbf{R}, t)$ and projects the product $f$ imposing that it is positive, namely, that the solution $\Psi$ has the same nodes (zeros) as the trial wave function $\Psi_{\mathrm{T}}$. One therefore solves the Schrödinger equation in the so-called fixednode approximation finding an upper bond to the true ground state. For a non-trivial excited state (not the lowest in its symmetry class), we are only ensured that we obtain the exact excited state if the trial wave function has the exact nodes.

\subsubsection{Density functional theory}

In density functional theory (DFT), the key quantity is the electron density $\rho(\mathbf{r})$ which is a function of three coordinates and therefore significantly simpler than the $3 \mathrm{~N}$-dimensional problem in wave-function-based methods. DFT is based on the Hohenberg and Kohn theorem (1964), which proves that the ground-state electron density uniquely determines the external potential. The density therefore determines the Hamiltonian, which in turn determines the wave functions and hence all electronic energies and other molecular properties of the system [19]. Hohenberg and Kohn also established the variational principle for the electron density: since only the exact ground-state electron density gives the true electronic energy, other density can only yield a higher electronic energy. The exact energy functional that delivers the ground-state energy is however unknown.

In practice, one makes use of the Kohn-Sham scheme [20] which maps the real interacting system with density $\rho$ to a non-interacting system with the same density. The mapping is in principle exact and results in the so-called Kohn-Sham equations:

$$
\left[-\frac{1}{2} \nabla^{2}+v_{\mathrm{eff}}([\rho] ; \mathbf{r})\right] \phi_{i}=\epsilon_{i} \phi_{i}
$$

where the ground-state electronic density is given by

$$
\rho(\mathbf{r})=\sum_{i=1}^{\text {occupied }}\left|\phi_{i}(\mathbf{r})\right|^{2} .
$$

The effective Kohn-Sham potential consists of three terms:

$$
v_{\mathrm{eff}}([\rho] ; \mathbf{r})=v_{\mathrm{ext}}(\mathbf{r})+\int \frac{\rho\left(\mathbf{r}^{\prime}\right)}{\left|\mathbf{r}-\mathbf{r}^{\prime}\right|} \mathrm{d} \mathbf{r}^{\prime}+v_{\mathrm{xc}}([\rho] ; \mathbf{r}),
$$

where $v_{\text {ext }}(\mathbf{r})$ is the external potential, followed by the Hartree potential and the exchange-correlation potential $v_{\mathrm{xc}}([\rho] ; \mathbf{r})$ which is defined as

$$
v_{\mathrm{xc}}([\rho] ; \mathbf{r}) \equiv \frac{\delta E_{\mathrm{xc}}[\rho]}{\delta \rho} .
$$


The exchange-correlation potential, $v_{\mathrm{xc}}([\rho] ; \mathbf{r})$, is the functional derivative of the exchange-correlation energy, $E_{\mathrm{xc}}[\rho]$, that enters in the expression of the total energy:

$$
\begin{aligned}
E= & -\frac{1}{2} \sum_{i=1}^{N} \int \phi_{i} \nabla^{2} \phi_{i} \mathrm{~d} \mathbf{r}+\int \rho(\mathbf{r}) v_{\text {ext }}(\mathbf{r}) \mathrm{d} \mathbf{r} \\
& +\frac{1}{2} \iint \frac{\rho(\mathbf{r}) \rho\left(\mathbf{r}^{\prime}\right)}{\left|\mathbf{r}-\mathbf{r}^{\prime}\right|} \mathrm{d} \mathbf{r} \mathrm{d} \mathbf{r}^{\prime}+E_{\text {xc }}[\rho] .
\end{aligned}
$$

Even though Equation 2.26 is in principle exact, the exchange-correlation functional is the unknown component of DFT, which must be approximated in an actual calculation.

The earliest approximation is the local density approximation (LDA) based on the homogeneous electron gas [20]. The total exchange-correlation energy is written as

$$
E_{\mathrm{xc}}^{\mathrm{LDA}}[\rho]=\int \rho(\mathbf{r}) \epsilon_{\mathrm{xc}}^{\mathrm{hom}}(\rho(\mathbf{r})) \mathrm{d} \mathbf{r},
$$

where $\epsilon_{\mathrm{xc}}^{\mathrm{hom}}(\rho)$ is the exchange-correlation energy per electron of a homogeneous electron gas of density $\rho$. Thus, in the LDA, the exchange-correlation energy per electron of an inhomogeneous system at a spatial point of density $\rho(\mathbf{r})$ is approximated as the exchange-correlation energy per particle of the uniform electron gas of the same density.

To improve upon LDA, the generalized gradient approximation (GGA) introduces a dependence on the gradient of the electron density:

$$
E_{\mathrm{xc}}^{\mathrm{GGA}}[\rho]=\int \rho(\mathbf{r}) \epsilon_{\mathrm{xc}}^{\mathrm{GGA}}(\rho(\mathbf{r}),|\nabla \rho(\mathbf{r})|) \mathrm{d} \mathbf{r} .
$$

A widely used GGA exchange functional is the Becke's functional, $E_{x}^{\mathrm{B} 88}$, which has only one parameter fitted to obtain the exchange energies of noble gas atoms [21]. Combined with the Lee, Yang and Parr (LYP) correlation energy, $E_{c}^{\mathrm{LYP}}$, with four parameters fitted to the energy of a helium atom [22], one obtains the so-called BLYP GGA functional.

GGA functionals considerably improve the energy and geometrical parameters of molecular systems over the LDA (in particular the overbinding problem of LDA in molecular systems), and further agreement with experimental or the best theoretical data can be obtained by including a portion of exact exchange as:

$$
E_{\mathrm{xc}}^{\mathrm{hybrid}}[\rho]=\alpha\left(E_{\mathrm{x}}^{\mathrm{HF}}-E_{\mathrm{x}}^{\mathrm{GGA}}[\rho]\right)+E_{\mathrm{xc}}^{\mathrm{GGA}}[\rho] .
$$

where $E_{\mathrm{x}}^{\mathrm{HF}}$ is the Hartree-Fock exchange energy calculated with Kohn-Sham orbitals, and $\alpha$ is the amount of exact exchange that is parameterized semi-empirically. This is estimated to be in the range of $20 \%$ to $25 \%$ for thermochemistry [23] and $40 \%$ to $60 \%$ for kinetics [24] but the optimal contribution can of course vary depending on the type of molecule and application.

In this work, we will employ Becke's three-term hybrid [25] in the form of the B3LYP functional [26]

$$
E_{\mathrm{xc}}^{\mathrm{B} 3 \mathrm{LYP}}[\rho]=(1-a) E_{x}^{\mathrm{LDA}}[\rho]+a E_{x}^{\mathrm{HF}}+b E_{x}^{\mathrm{B} 88}[\rho]+c E_{c}^{\mathrm{LYP}}[\rho]+(1-c) E_{c}^{\mathrm{LDA}},
$$


with $a=0.2, b=0.72$, and $c=0.81$. We use B3LYP mainly to optimize the geometry of various systems in the gas phase and in solution. We also make use of meta-GGA functionals [27, 28], which include the second derivative of the electron density and a dependence on the kinetic energy density defined as

$$
\tau(\mathbf{r})=\frac{1}{2} \sum_{i}\left|\nabla \phi_{i}(\mathbf{r})\right|^{2}
$$

Minnesota functionals are a class of widely used meta-GGA functionals which are being actively developed to tackle specifically different properties [29-39]. Here, we employ the M06-2X functional [32], a hybrid version of meta-GGA that contains $54 \%$ of exact exchange, to explore the thermochemistry of thermal isomerization reactions.

Finally, we describe the $\omega$ B97X-D functional [40] which we also use to study the thermochemistry of photoswitches. This functional belongs to the range-separated hybrid family and also includes an empirical dispersion correction [41]. In a rangeseparated functional, the Coulomb interaction is split into a long-range (SR) and a short-range (LR) component as

$$
\frac{1}{r}=\frac{\operatorname{erf}(\omega r)}{r}+\frac{\operatorname{erfc}(\omega r)}{r}
$$

where $\omega$ is a constant that defines the range of the two parts. The $\omega \mathrm{B} 97 \mathrm{X}$ rangeseparated hybrid functional [42] takes the following form:

$$
E_{\mathrm{xc}}^{\omega \mathrm{B} 97 \mathrm{X}}[\rho]=E_{\mathrm{x}}^{\mathrm{LR}-\mathrm{HF}}+c_{x} E_{\mathrm{x}}^{\mathrm{SR}-\mathrm{HF}}+E_{\mathrm{x}}^{\mathrm{SR}-\mathrm{B} 97}[\rho]+E_{\mathrm{c}}^{\mathrm{B} 97}[\rho] .
$$

Both short- and long-range exact exchange contributions are used in the expression, with $\omega=0.3$ bohr $^{-1}$ and $c_{x}=0.158$. At large electron-electron distances, $100 \%$ of Hartree-Fock exchange is employed to ameliorate self-interaction errors [43,44]. The exchange energy at short range is primarily described by the B97 GGA functionals [23], and the correlation energy is fully accounted for by the B97 functional. We note that, if $\omega=0$, the $\omega \mathrm{B} 97 \mathrm{X}$ functional simply reduces to its parent B97.

To obtain the $\omega \mathrm{B} 97 \mathrm{X}-\mathrm{D}$ functional, an empirical atomic-pairwise dispersion correction $E_{\mathrm{disp}}$ is added to the $\omega \mathrm{B} 97 \mathrm{X}$ energy

$$
E_{\omega \mathrm{B} 97 \mathrm{X}-\mathrm{D}}=E_{\omega \mathrm{B} 97 \mathrm{X}}+E_{\mathrm{disp}}
$$

Since the semi-empirical parameters of the $\omega \mathrm{B} 97 \mathrm{X}$ functional are optimized in the absence of $E_{\text {disp }}$, the functional is reparameterized using the same diverse training set as for the original $\omega \mathrm{B} 97 \mathrm{X}$ functional. The resulting optimized parameters are $\omega=0.2 \mathrm{bohr}^{-1}$ and $c_{x}=0.222$.

\subsubsection{Thermochemistry}

To enable a direct comparison with experimental reaction energies and barrier heights at ambient conditions, we need to compute the molar total internal energy, $E_{m, \text { total }}$, 
which is the sum of the molar $a b$ initio internal energy $\varepsilon_{m, 0}$ and the molar internal thermal energy, $E_{m, \text { corr }}$, which includes thermal electronic and nuclear translational, rotational and vibrational contributions as

$$
\begin{aligned}
E_{m, \text { total }} & =\varepsilon_{m, 0}+E_{m, \text { corr }}, \\
E_{m, \text { corr }} & =E_{m, \mathrm{el}}+E_{m, \text { trans }}+E_{m, \text { rot }}+E_{m, \text { vib }} .
\end{aligned}
$$

Even if we work at very low temperatures, we must include the zero-point vibrational

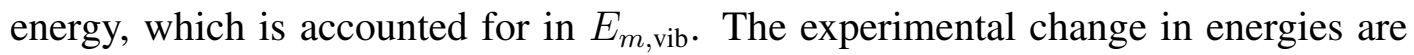
usually expressed in terms of the molar enthalpy, $H_{m}$, and the molar Gibbs free energy, $G_{m}$, which are related to the total internal energy as

$$
\begin{aligned}
& H_{m}=E_{m, \text { total }}+P V=E_{m, \text { total }}+R T, \\
& G_{m}=E_{m, \text { total }}+R T-T S_{m, \text { total }}=H_{m}-T S_{m, \text { total }},
\end{aligned}
$$

where we made use of the ideal gas assumption to express $H_{m}$ in term of the temperature $T$ with $R$ the ideal gas constant, and $S_{m \text {,total }}$ is the molar total entropy:

$$
S_{m, \text { total }}=S_{m, \mathrm{el}}+S_{m, \text { trans }}+S_{m, \text { rot }}+S_{m, \text { vib }}
$$

We briefly discuss here the procedure to compute the molar Gibbs free energy [45]. The system partition function, $Q(N, V, T)$, is related to the molecular partition function, $q(V, T)$, as

$$
Q(N, V, T)=\frac{q(V, T)^{N}}{N !},
$$

assuming that the molecules are independent. The molecular partition function is further approximated as the product of the partition function for the electronic and nuclear degrees of freedom as

$$
q=q_{\mathrm{el}} q_{\mathrm{trans}} q_{\mathrm{rot}} q_{\mathrm{vib}}
$$

where the electronic, translational, rotational and vibrational states are assumed to be independent. The molar internal thermal energy can then be expressed in term of molecular partition function, $q$, as

$$
E_{m, \mathrm{corr}}=R T^{2}\left(\frac{\partial \ln q}{\partial T}\right)_{V}
$$

and the total entropy contribution is given by

$$
\begin{aligned}
S_{\text {total }} & =k_{B} \ln Q+k_{B} T\left(\frac{\partial \ln Q}{\partial T}\right)_{V} \\
& =N k_{B} \ln q-k_{B} \ln N !+N k_{B} T\left(\frac{\partial \ln q}{\partial T}\right)_{V} .
\end{aligned}
$$

If one uses Stirling's approximation (i.e. $\ln N !=N \ln N-N$ ) then

$$
S_{\text {total }}=N k_{B}\left[1+\ln \left(\frac{q}{N}\right)+T\left(\frac{\partial \ln q}{\partial T}\right)_{V}\right] .
$$


The molar total entropy, $S_{m \text {,total }}$, is obtained by dividing $S_{\text {total }}$ with $n=N / N_{A}$, where $N_{A}$ is the Avogadro number. Using that $N_{A} k_{B}=R$, one obtains the following expression for one molecule $(N=1)$,

$$
S_{m, \text { total }}=R\left[\ln (q e)+T\left(\frac{\partial \ln q}{\partial T}\right)_{V}\right]
$$

where $e$ is the Euler's number.

We now need to compute the contribution of the electronic and nuclear motion to the molar internal thermal energy and the molar entropy. We start from the electronic partition function:

$$
q_{\mathrm{el}}=n_{0} \exp \left(-\frac{\varepsilon_{0}}{k_{B} T}\right)+n_{1} \exp \left(-\frac{\varepsilon_{1}}{k_{B} T}\right)+\cdots,
$$

where $n_{i}$ and $\varepsilon_{i}$ denote the degeneracy and the relative energy of the $i$-th state. Since our photoswitches absorb in the ultraviolet and visible regions, electronically excited states will not be occupied at ambient conditions, and thus, the electronic partition function simply becomes

$$
q_{\mathrm{el}}=n_{0},
$$

where we have taken $\epsilon_{0}$ to be the zero of the energy. Consequently, the internal thermal energy and entropy due to the electronic contribution are zero.

Next, the partition function for the nuclear translational motion is

$$
q_{\text {trans }}=\left(\frac{2 \pi m k_{B} T}{h^{2}}\right)^{3 / 2} V=\left(\frac{2 \pi m k_{B} T}{h^{2}}\right)^{3 / 2} \frac{k_{B} T}{P},
$$

where we also employ the ideal gas assumption. Since the partial derivation of $\ln q_{\text {trans }}$ with respect to $\mathrm{T}$ is

$$
\left(\frac{\partial \ln q_{\text {trans }}}{\partial T}\right)_{V}=\frac{3}{2 T}
$$

the molar internal thermal energy and entropy contribution from the nuclear translation become

$$
\begin{aligned}
E_{m, \text { trans }} & =R T^{2}\left(\frac{3}{2 T}\right)=1.5 R T \\
S_{m, \text { trans }} & =R\left[\ln \left(q_{\text {trans }} e\right)+T\left(\frac{3}{2 T}\right)\right]=R\left[\ln q_{\text {trans }}+2.5\right] .
\end{aligned}
$$

Moving to the nuclear rotational motion at ambient condition, the partition function for nonlinear polyatomic molecules without symmetry can be approximated as

$$
q_{\mathrm{rot}}=\sqrt{\pi}\left[\frac{T^{3 / 2}}{\left(\Theta_{\mathrm{rot}, x} \Theta_{\mathrm{rot}, y} \Theta_{\mathrm{rot}, z}\right)^{1 / 2}}\right] \text {, }
$$


where $\Theta_{\mathrm{rot}, A}=h^{2} /\left(8 \pi^{2} I_{A} k_{B}\right)$, and $I_{A}$ is the moment inertia with respect to the $A$ axis. Therefore, the molar internal thermal energy and entropy contributions from the nuclear rotation are

$$
\begin{aligned}
& E_{m, \mathrm{rot}}=R T^{2}\left(\frac{\partial \ln q_{\mathrm{rot}}}{\partial T}\right)_{V}=R T^{2}\left(\frac{3}{2 T}\right)=1.5 R T \\
& S_{m, \mathrm{rot}}=R\left[\ln q_{\mathrm{rot}}+T\left(\frac{\partial \ln q_{\mathrm{rot}}}{\partial T}\right)_{V}\right]=R\left[\ln q_{\mathrm{rot}}+1.5\right] .
\end{aligned}
$$

Finally, assuming that the bottom of the potential-energy well is the zero of the energy, the partition function for the nuclear vibrations under the harmonic approximation is

$$
q_{\mathrm{vib}}=\prod_{K} \frac{e^{-\Theta_{\mathrm{vib}, K} / 2 T}}{1-e^{-\Theta_{\mathrm{vib}, K} / T}},
$$

where $\Theta_{\mathrm{vib}, K}$ is the characteristic temperature for the vibrational mode $K$,

$$
\Theta_{\mathrm{vib}, K}=\frac{h \nu_{K}}{k_{B}} .
$$

Then, the molar internal thermal energy and entropy from the vibrational partition function become

$$
\begin{aligned}
E_{m, \mathrm{vib}} & =R \sum_{K} \Theta_{\mathrm{vib}, K}\left[0.5+\frac{1}{e^{-\Theta_{\mathrm{vib}, K} / T}-1}\right] \\
S_{m, \mathrm{vib}} & =R \sum_{K}\left[\frac{\Theta_{\mathrm{vib}, K} / T}{e^{\Theta_{\mathrm{vib}, K} / T}-1}-\ln \left(1-e^{-\Theta_{\mathrm{vib}, K} / T}\right)\right] .
\end{aligned}
$$

Having obtained the contribution of the nuclear motion to the molar internal thermal energy and entropy, we can compute the molar enthalpy and Gibbs free energy of the minimum and transition-state geometries according to Eqs. 2.37 and 2.38.

\subsection{Molecular mechanical simulations}

When moving to larger (bio)systems, quantum mechanical methods become quickly impractical, and the explicit consideration of electrons is often not necessary. In this case, one can resort to fully classical simulations, where the system is modeled at the level of atoms or even coarse-grained particles. In this dissertation, we will perform atomistic simulations to study the dynamical behavior of a protein.

\subsubsection{Classical force field}

A force field is an empirical energy function which describes the potential energy of a system of atoms in molecular mechanical simulations. The potential energy is given by the summation of a covalent and a noncovalent term as

$$
E_{\text {potential }}=E_{\text {covalent }}+E_{\text {noncovalent }},
$$


where the two terms are the result of several contributions:

$$
\begin{aligned}
E_{\text {covalent }} & =E_{\text {bond }}+E_{\text {angle }}+E_{\text {dihedral }} \\
E_{\text {noncovalent }} & =E_{\text {electrostatic }}+E_{\text {van der Waals }} .
\end{aligned}
$$

While electrons are not present in these expressions, their effect is implicit in all force-field terms. For instance, quantum mechanically, two atoms with high electronegativity index will rarely meet during a simulation due to the high electron density on both atoms. Molecular mechanically, these two atoms will possess considerably negative charges and repel each other electrostatically.

The bond stretching energy, $E_{\text {bond }}$, and bond-angle bending energy, $E_{\text {angle }}$, are commonly expressed as harmonic potentials in the bond length and angle,

$$
\begin{aligned}
& E_{\text {bond }}=\sum_{\text {bonds }} \frac{k_{b}}{2}\left(b-b_{0}\right)^{2} \\
& E_{\text {angle }}=\sum_{\text {angles }} \frac{k_{\theta}}{2}\left(\theta-\theta_{0}\right)^{2},
\end{aligned}
$$

where $k_{b}$ and $k_{\theta}$ are the force constants, and $b_{0}$ and $\theta_{0}$ are the equilibrium values of the bond length and angle, respectively. This harmonic approximation is customary in many force fields since the displacements are small at ambient conditions [46].

To account for the periodicity of the potential, the total dihedral energy expression contains a trigonometric function such as

$$
E_{\text {dihedral }}=\sum_{\phi} \sum_{n} V_{n}\left[1+\cos \left(n \phi-\gamma_{n}\right)\right],
$$

where $\phi$ is the angle between the $i j k$ and the $j k l$ planes in the $i-j-k-l$ system of atoms as shown in Figure 2.1a. The zero of the $\phi$ angle corresponds to the configuration where the $i$ and $l$ atoms are on the same side, $n$ is the periodicity, $V_{n}$ is the barrier for rotating around the dihedral with periodicity $360^{\circ} / n$, and $\gamma_{n}$ is the phase. Typically, a maximum of three terms is included in the expression of the dihedral potential.
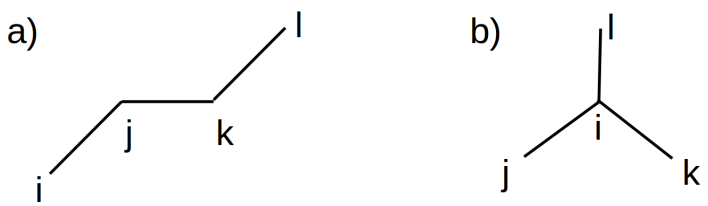

Figure 2.1: The 4-atom systems used to define a) the dihedral angle and b) the improper dihedral angle.

To illustrate the need for a periodic expression of the dihedral potential, we consider the example of an ethane molecule $\left(\mathrm{C}_{2} \mathrm{H}_{6}\right)$. As shown in Figure 2.2, rotation around the $\mathrm{C}-\mathrm{C}$ bond produces three staggered (minima) and three eclipsed (maxima) configurations. The corresponding potential-energy profile can be described very well by the expression (Eq. 2.59) with $n=3$ and $\gamma_{n}=0$. 
a)

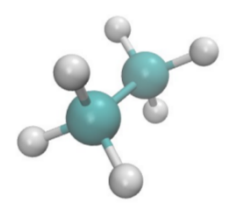

b)

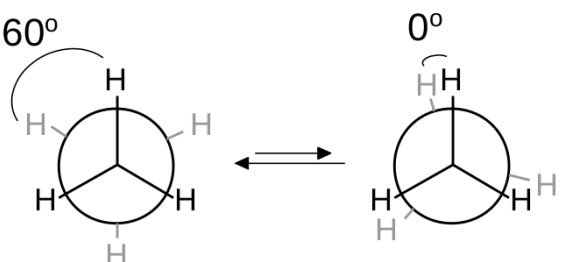

staggered eclipsed

c)

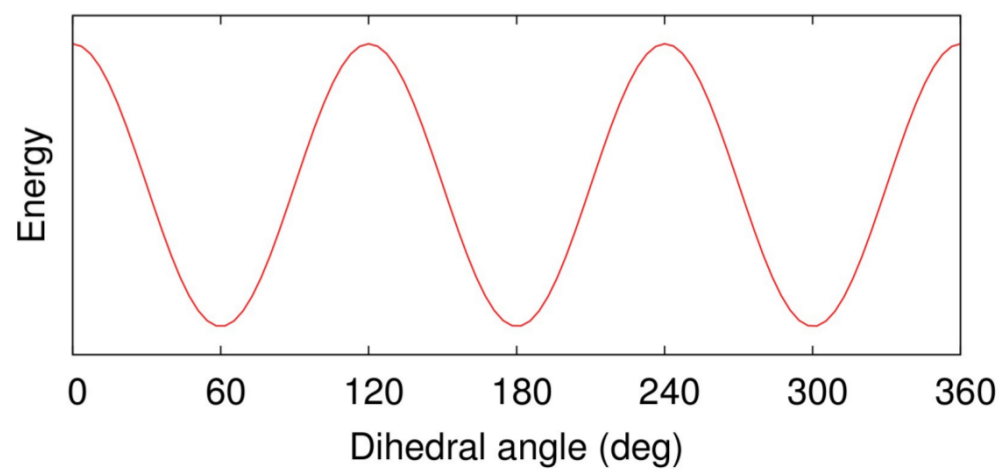

Figure 2.2: Periodicity of the dihedral angle potential. a) Optimal geometry of an ethane molecule $\left(\mathrm{C}_{2} \mathrm{H}_{6}\right)$, b) conformations of ethane which differ mainly in the dihedral angle, and c) sketch of the potential energy as a function of dihedral angle.

Furthermore, an improper dihedral term can be included in the expression of the force field to ensure planarity around a $s p^{2}$-hybridized atom. The improper dihedral angle $\zeta$ is defined as the angle between the $i j k$ and the $j k l$ planes but in the system where the $s p^{2}$-atom $i$ is connected covalently to the $j, k$ and $l$ atoms (see Figure 2.1b). The usual form of the total improper dihedral energy is

$$
E_{\text {improper }}=\sum_{\zeta} V_{\zeta}\left(\zeta-\zeta_{0}\right)^{2}
$$

where $\zeta_{0}$ is the equilibrium improper dihedral angle.

In our work, we employ a non-polarizable force field and use static point charges to describe the electrostatic behavior of the system. The interatomic electrostatic interaction contribution to the total potential energy is therefore formulated as

$$
E_{\text {electrostatic }}=\frac{1}{2} \frac{1}{4 \pi \epsilon_{0}} \sum_{i \neq j} \frac{q_{i} q_{j}}{\epsilon_{r}\left|\mathbf{R}_{i}-\mathbf{R}_{j}\right|},
$$

where $\epsilon_{r}$ is the relative dielectric constant, and $q_{i}$ and $q_{j}$ are the atomic charge of atoms $i$ and $j$ located at $\mathbf{R}_{i}$ and $\mathbf{R}_{j}$, respectively.

Finally, the total van der Waals energy is accounted for via Lennard-Jones interactions:

$$
E_{\text {van der Waals }}=\frac{1}{2} \sum_{i \neq j} 4 \varepsilon_{i j}\left[\left(\frac{\sigma_{i j}}{r_{i j}}\right)^{12}-\left(\frac{\sigma_{i j}}{r_{i j}}\right)^{6}\right],
$$

where $\varepsilon_{i j}$ is the depth of the potential well, and $\sigma_{i j}$ the finite distance at which the potential for particles $i$ and $j$ is zero. We note that the electrostatic and van der Waals 
terms are calculated not only for pairs of atoms that are noncovalently bound but also for two atoms that are bridged covalently by at least three consecutive atoms, since their interactions are not accounted for by any of the covalent terms.

Computationally, the evaluation of the noncovalent terms is the bottleneck to obtain the total potential energy because the number of atomic pairs grows quadratically with the system size, whereas the covalent term scales nearly linearly [47]. Fortunately, one can truncate the number of computed interactions based on spatial consideration since faraway atoms weakly interact with each other: Van der Waals interactions decay rapidly due to the dependence on the $r^{-6}$ and $r^{-12}$, enabling the evaluation of the interaction only for the region where the interatomic distance is less than a chosen cutoff. On the other hand, electrostatic interactions decay slowly and need to be treated beyond the cutoff, for instance, with the particle mesh Ewald method [48].

We employ here the Amber force field to simulate a protein in aqueous solution. We note that special care has to be taken in choosing the force-field version, especially when one deals with proteins that are mostly composed of $\alpha$-helix secondary structures. The original Amber94 force field and its subsequent Amber99 modification tend to overstabilize the $\alpha$-helices, probably due to insufficiencies in the fitting procedure where only a small number of short-peptide conformers was considered $[49,50]$. In subsequent versions, particular effort was devoted to reparameterize the dihedral potentials because of their crucial role in determining the relative energies of conformers and barrier heights for conformational changes. In the Amber99SB force field, the $\phi$ and $\psi$ dihedral terms of the backbone were reparameterized by fitting the energies of all conformations of glycine and alanine tetrapeptides to high-level quantum mechanical calculations [51]. Then, two different routes were parallelly followed to further improve the force field. The Amber99SB* force field was developed by adding an additional term to the existing dihedral potentials of the backbone in order to reproduce the fraction of $\alpha$-helix in short peptides comprising five and six amino-acid residues as measured in NMR experiments [52]. The other effort was focused on the dihedral term of four sidechains, namely, isoleucine, leucine, aspartate and asparagine, yielding the Amber99SB-ILDN force field [53]. The fitting was performed to mimic the energies of the multiple conformers of these sidechains at the density-fitted-linear-MP2/aug-cc-pVTZ quantum mechanical level. The refined force field was validated against hundreds of experimentally-determined protein structures containing these four sidechains. By combining these two strategies, one obtains the Amber99SB*-ILDN force field which is found to perform very well $[54,55]$ and is therefore employed in our work.

\subsubsection{Molecular dynamics}

In molecular dynamics (MD) simulations, we solve Newton's equations for a system of $\mathrm{N}$ interacting atoms

$$
m_{i} \frac{\mathrm{d}^{2} \mathbf{r}_{i}}{\mathrm{dt}^{2}}=\mathbf{F}_{i}=-\nabla_{i} E_{\text {potential }}
$$


MD simulations are commonly used to study the thermodynamical and dynamical properties of a system by propagating the positions and velocities of the atoms. When applied to protein systems, MD techniques can be used to characterize the fluctuations of the binding pockets, the protein-ligand interactions, the conformational changes leading to protein folding, etc.

To start a MD simulation, one needs a reasonable set of positions for the atoms. For proteins, the starting coordinates can be taken from an X-ray structure which is however often incomplete due to the difficulties of locating flexible non-hydrogen atoms and to the absence of hydrogen atoms because of their weak scattering of $\mathrm{X}$-ray irradiation. Therefore, one has to complement these missing atoms before performing any simulations. In particular, hydrogen atoms should be added carefully since the protonation state of charged amino-acid residues is strongly influenced by the acidity of the solution and the character of neighboring residues. For instance, a solvent-exposed glutamate residue at a neutral $\mathrm{pH}$ is deprotonated but, if it is adjacent to an aspartate residue which has a lower $\mathrm{pKa}$ value, the glutamate anion will be protonated.

Deducing the protonation state of amino-acid residues is very important because it affects the stability of noncovalent interactions among amino-acid sidechains and also determines directly the total charge of the protein and, consequently, the tertiary and quarternary structures of (some) proteins. An amino acid which requires special attention is the cysteine residue which can be found either in a single form, bearing a thiol (-SH) group, or in an oxidized, dimerized form where two cysteines are connected covalently. The resulting disulfide $(-\mathrm{S}-\mathrm{S}-)$ bond is crucial in maintaining the tertiary structure of a protein because it keeps two secondary structures within a particular range of distance.

Once all missing atoms have been added, the protein is solvated in a simulation box. The simulations are typically performed using periodic boundary conditions where the simulated system is placed in a unit cell and periodically repeated. The total charge of the simulation cell should be zero to avoid unnatural electrostatic interactions between cells. The neutrality of a cell is achieved by adding appropriate counterions (e.g. $\mathrm{Na}^{+}$or $\mathrm{Cl}^{-}$) to the protein solution. Finally, the geometry of the system is optimized to avoid the presence of atoms too close to each other, which would result in a very high kinetic energy when the simulation is started.

To begin the simulation, the initial velocities of the atoms are generated according to the Maxwell-Boltzmann distribution so that

$$
P(v)=\left(\frac{m}{2 \pi k_{B} T}\right)^{3 / 2} 4 \pi v^{2} \exp \left(-\frac{m v^{2}}{2 k_{B} T}\right),
$$

where $v=\sqrt{v_{x}^{2}+v_{y}^{2}+v_{z}^{2}}$.

Next, an integrator is needed to propagate the positions and the velocities of the atoms. In our simulations, we employ the leap-frog algorithm which uses positions and velocities computed at different times [56]. To derive the leap-frog algorithm, we start from the differential expression of the acceleration as

$$
\mathbf{a}(t)=\frac{\mathbf{v}(t+\Delta t)-\mathbf{v}(t-\Delta t)}{2 \Delta t} .
$$


For a more accurate $\mathbf{a}(t)$, a half timestep is employed, and having rearranged the equation, we arrive at the following expression for the velocity,

$$
\mathbf{v}(t+0.5 \Delta t)=\mathbf{v}(t-0.5 \Delta t)+\frac{\mathbf{F}(t)}{m} \Delta t .
$$

To determine the next position, $\mathbf{r}(t+\Delta t)$, we begin with a basic definition of the velocity (equivalent to Eq. 2.65), rewrite it again in terms of a half timestep and shift the time by $0.5 \Delta t$ in order to make use of the expression for $\mathbf{v}(t+0.5 \Delta t)$. The resulting formulation is

$$
\mathbf{r}(t+\Delta t)=\mathbf{r}(t)+\mathbf{v}(t+0.5 \Delta t) \Delta t .
$$

We note that a drawback of the algorithm is that distances and velocities are not available at the same time $t$. Therefore, to compute the kinetic energy at the same time as the potential, one estimates the velocity from the following relation:

$$
\mathbf{v}(t)=\frac{\mathbf{v}(t+0.5 \Delta t)+\mathbf{v}(t-0.5 \Delta t)}{2} .
$$

It is also crucial to appropriately choose the timestep, $\Delta t$, for the given system as a small timestep results in a slow propagation of the trajectories, whereas a very big timestep leads to instabilities when two atoms come close to each other.

Molecular dynamics simulations usually consist of two consecutive steps, namely, a preparation and a production run. In the preparation step, the system is first thermally equilibrated in the NVT ensemble and its density is then adjusted by allowing the volume to change in the NPT ensemble. Afterwards, the simulation enters the production phase using the ensemble of interest, and the analysis is then performed on the trajectories obtained in this last step.

\subsection{Computational details}

The computational codes used in this dissertation are listed here.

- Molcas $[57,58]$ is a quantum chemical code specialized in multiconfigurational methods. We employ this code to perform CASSCF and CASPT2 calculations.

- Gaussian 09 [59] is an electronic structure program that we use to calculate the optimal ground-state geometry, transition states, intrinsic reaction paths, thermochemistry quantities and charge analysis.

- GAMESS $[60,61]$ is also a code for molecular quantum chemistry which we use to generate multiconfigurational and generalized-valence-bond wave functions.

- CHAMP is a quantum Monte Carlo suite of programs for electronic structure calculations of atomic and molecular systems which is employed to perform VMC and DMC calculations. 
- AutoDock [62] is a suite of automated docking tools which is here used to predict how small molecules bind covalently or noncovalently to a given protein.

- Ambertools [63] is employed to derive atomic charges for molecular mechanical simulation.

- Gromacs [64] is a package to perform atomistic molecular dynamics simulations of biomolecules. We simulate here a protein in aqueous solution and analyze the resulting trajectories with this code.

- VMD [65] is a visualization program mainly used for displaying trajectories and performing cluster analysis of multiple simulation snapshots. 


\section{Bibliography}

[1] M. Born and R. Oppenheimer, Annalen der Physik 389, 457 (1927).

[2] J. C. Slater, Phys. Rev. 34, 1293 (1929).

[3] C. C. J. Roothaan, Rev. Mod. Phys. 23, 69 (1951).

[4] B. O. Roos, P. R. Taylor, and P. E. M. Siegbahn, Chem. Phys. 48, 157 (1980).

[5] B. O. Roos, Int. J. Quantum Chem. 18, 175 (1980).

[6] C. Møller and M. S. Plesset, Phys. Rev. 46, 618 (1934).

[7] K. Andersson, P. A. Malmqvist, B. O. Roos, A. J. Sadlej, and K. Wolinski, J. Phys. Chem. 94, 5483 (1990).

[8] K. Andersson, P.-Å. Malmqvist, and B. O. Roos, J. Chem. Phys. 96, 1218 (1992).

[9] G. Ghigo, B. O. Roos, and P.-Å. Malmqvist, Chem. Phys. Lett. 396, 142 (2004).

[10] M. Schreiber, M. R. Silva-Junior, S. P. Sauer, and W. Thiel, J. Chem. Phys. 128, 134110 (2008).

[11] C. J. Cramer and B. A. Smith, J. Phys. Chem. 100, 9664 (1996).

[12] H.-J. Werner, Mol. Phys. 89, 645 (1996).

[13] D. Grabarek, E. Walczak, and T. Andruniów, J. Chem. Theory Comput. 12, 2346 (2016).

[14] A. Lüchow, WIREs: Comput. Mol. Sci. 1, 388 (2011).

[15] N. Metropolis, A. W. Rosenbluth, M. N. Rosenbluth, A. H. Teller, and E. Teller, J. Chem. Phys. 21, 1087 (1953).

[16] C. Umrigar, Phys. Rev. Lett. 71, 408 (1993).

[17] C. Umrigar, J. Toulouse, C. Filippi, S. Sorella, and R. G. Hennig, Phys. Rev. Lett. 98, 110201 (2007). 
[18] C. Filippi, M. Zaccheddu, and F. Buda, J. Chem. Theory Comput. 5, 2074 (2009).

[19] P. Hohenberg and W. Kohn, Phys. Rev. 136, B864 (1964).

[20] W. Kohn and L. J. Sham, Phys. Rev. 140, A1133 (1965).

[21] A. D. Becke, Phys. Rev. A 38, 3098 (1988).

[22] C. Lee, W. Yang, and R. G. Parr, Phys. Rev. B 37, 785 (1988).

[23] A. D. Becke, J. Chem. Phys. 107, 8554 (1997).

[24] A. D. Boese and J. M. Martin, J. Chem. Phys. 121, 3405 (2004).

[25] A. D. Becke, J. Chem. Phys. 98, 5648 (1993).

[26] P. Stephens, F. Devlin, C. Chabalowski, and M. J. Frisch, J. Phys. Chem. 98, 11623 (1994).

[27] S. K. Ghosh and R. G. Parr, Phys. Rev. 34, 785 (1986).

[28] A. Becke and M. Roussel, Phys. Rev. A 39, 3761 (1989).

[29] Y. Zhao and D. G. Truhlar, J. Phys. Chem. A 109, 5656 (2005).

[30] Y. Zhao and D. G. Truhlar, J. Chem. Phys. 125, 194101 (2006).

[31] Y. Wang, X. Jin, S. Y. Haoyu, D. G. Truhlar, and X. He, Proc. Natl. Acad. Sci. 114, 8487 (2017).

[32] Y. Zhao and D. G. Truhlar, Theor. Chem. Acc. 120, 215 (2008).

[33] Y. Zhao and D. G. Truhlar, J. Chem. Theory Comput. 4, 1849 (2008).

[34] R. Peverati and D. G. Truhlar, J. Phys. Chem. Lett. 3, 117 (2011).

[35] R. Peverati and D. G. Truhlar, J. Phys. Chem. Lett. 2, 2810 (2011).

[36] R. Peverati and D. G. Truhlar, Phys. Chem. Chem. Phys. 14, 16187 (2012).

[37] R. Peverati and D. G. Truhlar, Phys. Chem. Chem. Phys. 14, 13171 (2012).

[38] S. Y. Haoyu, X. He, S. L. Li, and D. G. Truhlar, Chem. Sci. 7, 5032 (2016).

[39] H. S. Yu, X. He, and D. G. Truhlar, J. Chem. Theory Comput. 12, 1280 (2016).

[40] J.-D. Chai and M. Head-Gordon, Phys. Chem. Chem. Phys. 10, 6615 (2008).

[41] S. Grimme, J. Comput. Chem. 27, 1787 (2006).

[42] J.-D. Chai and M. Head-Gordon, J. Chem. Phys. 128, 084106 (2008). 
[43] P. M. Gill, R. D. Adamson, and J. A. Pople, Mol. Phys. 88, 1005 (1996).

[44] Y. Tawada, T. Tsuneda, S. Yanagisawa, T. Yanai, and K. Hirao, J. Chem. Phys. 120, 8425 (2004).

[45] J. W. Ochterski, Thermochemistry in gaussian (Gaussian, Inc, 2000).

[46] B. R. Brooks, R. E. Bruccoleri, B. D. Olafson, D. J. States, S. Swaminathan, and M. Karplus, J. Comput. Chem. 4, 187 (1983).

[47] A. R. Leach, Molecular modelling: principles and applications (Pearson education, 2001).

[48] T. Darden, D. York, and L. Pedersen, J. Chem. Phys. 98, 10089 (1993).

[49] A. Okur, B. Strockbine, V. Hornak, and C. Simmerling, J. Comput. Chem. 24, 21 (2003).

[50] A. E. Garcia and K. Y. Sanbonmatsu, Proc. Natl. Acad. Sci. 99, 2782 (2002).

[51] V. Hornak, R. Abel, A. Okur, B. Strockbine, A. Roitberg, and C. Simmerling, Proteins 65, 712 (2006).

[52] R. B. Best and G. Hummer, J. Phys. Chem. B 113, 9004 (2009).

[53] K. Lindorff-Larsen, S. Piana, K. Palmo, P. Maragakis, J. L. Klepeis, R. O. Dror, and D. E. Shaw, Proteins 78, 1950 (2010).

[54] E. A. Cino, W.-Y. Choy, and M. Karttunen, J. Chem. Theory Comput. 8, 2725 (2012).

[55] K. Lindorff-Larsen, P. Maragakis, S. Piana, M. P. Eastwood, R. O. Dror, and D. E. Shaw, PloS one 7, e32131 (2012).

[56] R. Hockney, S. Goel, and J. Eastwood, J. Comput. Phys. 14, 148 (1974).

[57] F. Aquilante, L. De Vico, N. Ferré, G. Ghigo, P.-Å. Malmqvist, P. Neogrády, T. B. Pedersen, M. Pitoňák, M. Reiher, B. O. Roos, L. Serrano-Andrés, M. Urban, V. Veryazov, and R. Lindh, J. Comput. Chem. 31, 224 (2010).

[58] F. Aquilante, J. Autschbach, R. K. Carlson, L. F. Chibotaru, M. G. Delcey, L. De Vico, I. Fdez. Galván, N. Ferré, L. M. Frutos, L. Gagliardi, M. Garavelli, A. Giussani, C. E. Hoyer, G. Li Manni, H. Lischka, D. Ma, P. Å. Malmqvist, T. Müller, A. Nenov, M. Olivucci, T. B. Pedersen, D. Peng, F. Plasser, B. Pritchard, M. Reiher, I. Rivalta, I. Schapiro, J. Segarra-Martí, M. Stenrup, D. G. Truhlar, L. Ungur, A. Valentini, S. Vancoillie, V. Veryazov, V. P. Vysotskiy, O. Weingart, F. Zapata, and R. Lindh, J. Comput. Chem. 37, 506 (2016). 
[59] M. J. Frisch, G. W. Trucks, H. B. Schlegel, G. E. Scuseria, M. A. Robb, J. R. Cheeseman, G. Scalmani, V. Barone, B. Mennucci, G. A. Petersson, H. Nakatsuji, M. Caricato, X. Li, H. P. Hratchian, A. F. Izmaylov, J. Bloino, G. Zheng, J. L. Sonnenberg, M. Hada, M. Ehara, K. Toyota, R. Fukuda, J. Hasegawa, M. Ishida, T. Nakajima, Y. Honda, O. Kitao, H. Nakai, T. Vreven, J. A. Montgomery, Jr., J. E. Peralta, F. Ogliaro, M. Bearpark, J. J. Heyd, E. Brothers, K. N. Kudin, V. N. Staroverov, R. Kobayashi, J. Normand, K. Raghavachari, A. Rendell, J. C. Burant, S. S. Iyengar, J. Tomasi, M. Cossi, N. Rega, J. M. Millam, M. Klene, J. E. Knox, J. B. Cross, V. Bakken, C. Adamo, J. Jaramillo, R. Gomperts, R. E. Stratmann, O. Yazyev, A. J. Austin, R. Cammi, C. Pomelli, J. W. Ochterski, R. L. Martin, K. Morokuma, V. G. Zakrzewski, G. A. Voth, P. Salvador, J. J. Dannenberg, S. Dapprich, A. D. Daniels, Ö. Farkas, J. B. Foresman, J. V. Ortiz, J. Cioslowski, and D. J. Fox, Gaussian 09 Revision D.01, gaussian Inc. Wallingford CT 2009.

[60] M. W. Schmidt, K. K. Baldridge, J. A. Boatz, S. T. Elbert, M. S. Gordon, J. H. Jensen, S. Koseki, N. Matsunaga, K. A. Nguyen, S. Su, T. L. Windus, M. Dupuis, and J. A. Montgomery, J. Comput. Chem. 14, 1347 (1993).

[61] M. S. Gordon and M. W. Schmidt, in Theory and Applications of Computational Chemistry: the first forty years, edited by C. Dykstra, G. Frenking, K. Kim, and G. Scuseria (Elsevier, 2011), Chap. 41, pp. 1167-1190.

[62] G. M. Morris, R. Huey, W. Lindstrom, M. F. Sanner, R. K. Belew, D. S. Goodsell, and A. J. Olson, J. Comput. Chem. 30, 2785 (2009).

[63] D. A. Case, T. A. Darden, T. E. Cheatham, C. L. Simmerling, J. Wang, R. E. Duke, R. Luo, R. C. Walker, W. Zhang, K. M. Merz, B. Roberts, S. Hayik, A. Roitberg, G. Seabra, J. Swails, A. W. Goetz, I. Kolossváry, K. F. Wong, F. Paesani, J. Vanicek, R. M. Wolf, J. Liu, X. Wu, S. R. Brozell, T. Steinbrecher, H. Gohlke, Q. Cai, X. Ye, J. Wang, M. J. Hsieh, G. Cui, D. R. Roe, D. H. Mathews, M. G. Seetin, R. Salomon-Ferrer, C. Sagui, V. Babin, T. Luchko, S. Gusarov, A. Kovalenko, and P. A. Kollman, AMBER 12, 2012.

[64] D. Van Der Spoel, E. Lindahl, B. Hess, G. Groenhof, A. E. Mark, and H. J. Berendsen, J. Comput. Chem. 26, 1701 (2005).

[65] W. Humphrey, A. Dalke, and K. Schulten, J. Mol. Graph. 14, 33 (1996). 


\section{Chapter 3}

\section{Multiple-resonance local wave functions for accurate excited states in quantum Monte Carlo ${ }^{\dagger}$}

We introduce a novel class of local multideterminant Jastrow-Slater wave functions for the efficient and accurate treatment of excited states in quantum Monte Carlo. The wave function is expanded as a linear combination of excitations built from multiple sets of localized orbitals that correspond to the bonding patterns of the different Lewis resonance structures of the molecule. We capitalize on the concept of orbital domains of local coupled-cluster methods, which is here applied to the active space, to select the orbitals to correlate and construct the important transitions. The excitations are further grouped into classes, which are ordered in importance and can be systematically included in the Jastrow-Slater wave function to ensure a balanced description of all states of interest. We assess the performance of the proposed wave function in the calculation of vertical excitation energies and optimal excitedstate geometries of retinal models whose $\pi \rightarrow \pi^{*}$ state has a strong intramolecular charge-transfer character. We find that our multi-resonance wave functions recover the reference values of the total energies of the ground and excited states with only a small number of excitations and that the same expansion can be flexibly used at very different geometries. Furthermore, significant computational saving can also be gained in the orbital optimization step by selectively mixing occupied and virtual orbitals based on spatial considerations without loss of accuracy on the excitation energy. Our multi-resonance wave functions are therefore compact, accurate and very promising for the calculation of multiple excited states of different character in large molecules.

\footnotetext{
${ }^{\dagger}$ This chapter has been published as H. Zulfikri, C. Amovilli, and C. Filippi, "Multiple-resonance local wave functions for accurate excited states in quantum Monte Carlo", J. Chem. Theory Comput. 2016, 12(3), 1157-1168
} 


\subsection{Introduction}

The development of local correlation methods to describe multiple electronic states of extended molecular systems is a very active and exciting research area in computational chemistry. By expressing the wave function in a local orbital basis, one can make use of the assumption that electron correlation is a local phenomenon for instance for nonmetallic systems in the ground state [1]. In so doing, a local occupied orbital or an orbital pair only correlates to a subset of local virtual orbitals selected on the basis of certain criteria such as spatial considerations [2,3], the threshold on the magnitude of the exchange integrals [4], or the threshold on the occupation number of virtual pair natural orbitals $[5,6]$. A local approach has clear advantages over traditional methods using delocalized orbitals, where all occupied orbitals correlate to the virtual ones. For the ground state, the local scheme has been implemented in several single-reference methods such as the configuration-interaction [7-9], coupledcluster $[2,3,6,10,11]$, and perturbation [12-15] approaches as well as in various multi-reference methods [4,16-21]. The extension of the local ansatz to excited states is however not straightforward since the excited state may be characterized by non-local transitions and, importantly, multiple states have to be treated at the same level of accuracy [16,22-27].

Quantum Monte Carlo (QMC) methods which provide a stochastic approach to the solution of the many-body problem, can also benefit from the use of local orbitals. In the commonly employed Jastrow-Slater wave functions, the Jastrow factor recovers dynamic correlation whereas static correlation is addressed through a symmetryadapted linear combination of Slater determinants which can be built out of local or delocalized orbitals. To construct the determinantal component, a set of relevant orbitals is selected and often correlated in a (nearly) complete active space (CAS) expansion to capture the desired chemical features in the application of interest. Unfortunately, the dimension of such an expansion grows dramatically (exponentially in the CAS case) with the system size and hence hinders its application to large molecules. As the system grows, truncation of the expansion is therefore inevitable and a simple procedure as the use of a threshold on the coefficients does not guarantee the same expansion size at different geometries. Consequently, it becomes difficult to maintain an affordable number of determinants and ensure a balanced description of different parts of the potential energy surface of all the states under consideration.

The availability of accurate Jastrow-Slater wave functions with a small and transferable determinantal component is therefore highly desirable. In previous work with Fracchia, we have shown that this is possible in QMC with the use of orbitals localized over one or two centers and a local correlation scheme, which allows us to progressively construct accurate and compact multi-determinant wave functions of increasing quality. These wave functions were successfully applied to the ground state of closed and open shell systems [28-30]. By correlating two pairs of electrons sitting in adjacent bonding orbitals with the corresponding anti-bonding orbitals, we achieved linear scaling and, consequently, a significant computational gain with respect to other local expansions also employed in QMC as in the perfect- 
pair generalized-valence-bond [31] or the valence-bond approach built on atomiccentered orbitals [32].

In general, however, our ground-state scheme breaks down for excited states since transitions between orbitals separated by several bonds may acquire significant weight in the many-body wave function if the excited state has charge-transfer or Rydberg character. The first important step is therefore to understand how to select which orbitals we want to correlate to account for the most important determinants in the ground and/or the excited states. To this aim, we capitalize on the concept of orbital domains of local coupled-cluster methods [2,3,22-24] which we apply here to the local orbitals in the active space. We further classify the excitations in classes and include them progressively into the expansion of the Jastrow-Slater wave functions so that multiple states of different character are treated on an equal footing. Such a procedure allows us to control what enters in our wave functions but the key step to ensure a compact and transferable determinantal expansion is to also account for the multiple Lewis resonance structures of the molecule, whose relative importance may change in different structural configurations or electronic states. We therefore construct the domains and excitation classes for all sets of local orbitals corresponding to the multiple resonance structures and write the wave function as a linear combination of the resulting determinants. We demonstrate the excellent performance of our compact multi-resonance local Jastrow-Slater wave functions in the calculation of the vertical excitation and geometry optimization of the charge-transfer $\pi \rightarrow \pi^{*}$ state of retinal model chromophores.

The subsequent part of this chapter is organized as follow. In Section 3.2, we illustrate the concept of orbital domains and excitation classes for multiple resonance structures. The computational details are presented in Section 3.3 and the performance of our method is demonstrated in Section 3.4. We conclude in Section 3.5.

\subsection{Method}

To illustrate the potential of a local approach in QMC, we briefly describe the linearscaling scheme we recently introduced for ground states, which couples electron pairs locally to progressively construct wave functions of increasing quality [28-30]. In our approach, the occupied orbitals in the reference are localized over one or two centers along with the corresponding antibonding orbitals in the virtual space. The determinants are then generated by correlating two pairs of electrons sitting in adjacent bonding orbitals and the corresponding two antibonding orbitals in a so-called CAS $(4,4)$ expansion (i.e. by including the excitations in the active space obtained by correlating four electrons in the two bonding and two antibonding orbitals). Importantly, we demonstrated in our previous work that it is possible to classify the resulting transitions in classes of increasing importance and, in so doing, building a set of modular wave functions of growing complexity. Since electrons located in separated orbitals are not correlated, the number of determinants in the wave function scales linearly with the size of the molecule. 
To understand the difficulties of extending our local scheme to excited states and how we will solve them, we consider the retinal protonated Schiff base chromophore with three conjugated $\pi$ bonds (PSB3) of Figure 3.1, which, despite its apparent simplicity, represents a challenging model for the study of retinal photochemistry. This molecule well exemplifies the complications occurring in the excited state since its $\pi \rightarrow \pi^{*}$ excitation is accompanied by significant transfer of electronic charge from the carbon to the nitrogen terminus (or, equivalently, of positive charge in the opposite direction).

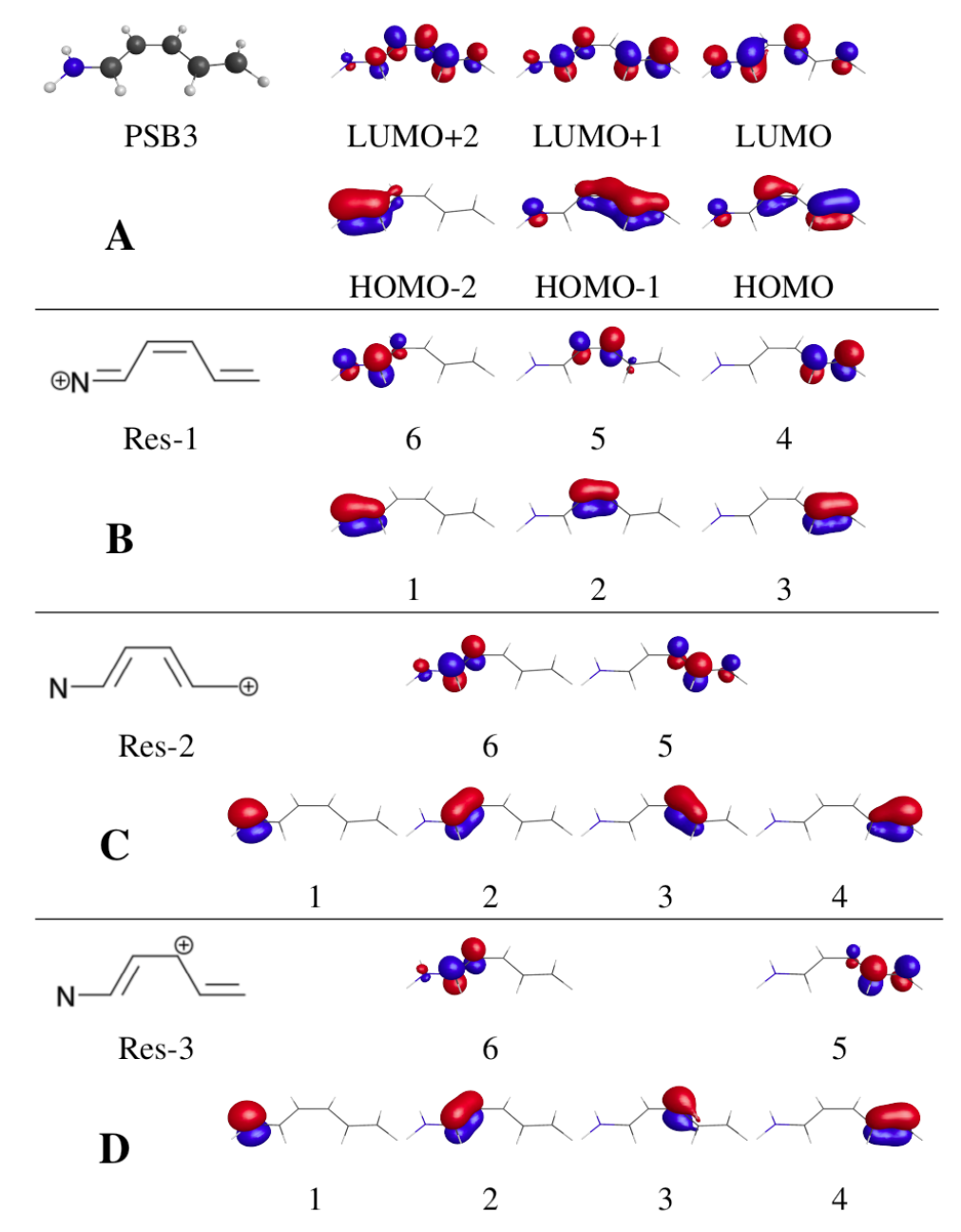

Figure 3.1: PSB3: (A) Active delocalized $\pi$ orbitals obtained at the CASSCF $(6,6)$ level, and (B-D) three unitary transformed sets of localized orbitals corresponding to the three Lewis resonance structures.

To account in a balanced manner for static correlation in the ground and excited states, one could adopt a delocalized description in terms of three delocalized $\pi$ bonding and three $\pi^{*}$ antibonding orbitals (panel A) and correlate the six electrons in the resulting active space, constructing a minimal $\mathrm{CAS}(6,6)$ expansion. In addition to this standard representation of the determinantal component of the wave function, we can however construct by unitary transformation not one but three equivalent sets 
of localized $\pi$ orbitals, corresponding to the three possible Lewis resonance structures. These different local orbital pictures will be more suitable to describe the electronic structure of PSB3 at different geometries or in different states: While the first resonance structure (Res-1) is dominant at the ground-state equilibrium, we anticipate that the other resonance structures will be very important in the treatment of the $\pi \rightarrow \pi^{*}$ excitation with its strong charge-transfer character. In general, all resonance structures will be visited in different regions of the excited-state potential energy surface. We stress that, if we include all excitations in the active space, these orbital representations are equivalent and one will recover the same amount of electronic correlation. The goal here is however to find a representation and a set of rules which result in a compact wave function without including all excitations, given their exponentially growing number with system size.

It is rather straightforward to understand the failure of our ground-state local correlation scheme if we focus on the dominant Lewis structure at equilibrium (panel B) and on a multi-determinant expansion on the corresponding local orbitals. As discussed below, an analysis of the dominant single-electron transitions reveals that spatially remote orbitals are coupled (e.g. bonding orbital 3 with antibonding orbital 6). Consequently, we cannot adopt the set of rules described above for ground states since it is impossible to simply correlate adjacent pairs of bonding/antibonding orbitals.

\subsubsection{Domains and excitation classes}

To select the orbitals to correlate, we borrow the concept of orbital domains from local-correlation methods $[1-3,7,22-24]$ and adapt it to the advantageous features of quantum Monte Carlo. Here, we define a domain as an orbital in the reference and the set of orbitals which are coupled to it by a large single-electron transition in the multi-determinant wave functions of the states of interest. As threshold on the weight to select the important singles, we choose to use the highest weight in any of the states under consideration so that all orbitals in the active space appear at least in one single excitation. In building our multi-determinant wave functions, we will then only retain the domains characterized by large single transitions and generate the double and higher excitations within and from the union of the surviving domains. Differently from conventional local schemes in quantum chemistry, we will construct the domains only from the orbitals within the active space (letting the Jastrow factor and, subsequently, diffusion Monte Carlo recover dynamical correlation) and also attempt to further classify the resulting excitations to generate a set of wave functions of increasing complexity as we had done for ground states.

To illustrate our scheme and investigate whether it can lead to a balanced description of the ground and excited states, we first focus on PSB3 at equilibrium and the Res-1 local orbital representation (Figure 3.1), and compute the CASSCF wave functions correlating the three bonding orbitals (labeled 1,2,3) and three antibonding orbitals (labeled 4,5,6) in a CAS $(6,6)$ expansion. Through the analysis of weights of the configurations state functions entering the wave function, we can identify whether the subset of configurations generated with the use of orbital domains recovers most 
of the weights of the wave functions in both states and which excitation classes may potentially be neglected when using such determinantal component in a QMC calculation.

If one defines a reference configuration as a configuration in the ground state consisting of doubly-occupied localized bonding orbitals, there is only one such configuration for the Res-1 representation of PSB3, namely 222000, where a configuration is denoted as $\mathrm{n}_{1} \mathrm{n}_{2} \mathrm{n}_{3} \mathrm{n}_{4} \mathrm{n}_{5} \mathrm{n}_{6}$ with $\mathrm{n}_{m}$ the occupation number of orbital $m$. To build the domains, we now consider the single excitations out of the reference in the Res1 wave function of the $\pi \rightarrow \pi^{*}$ state and find that, as reported in Table 3.1, there are several single-electron transitions carrying significant weight, differently to what one would obtain in an expansion over delocalized orbitals where the HOMO-LUMO transition is instead dominant. Due to the charge-transfer nature of the excited state, the single excitations connect the bonding to the antibonding orbitals sitting on their left and grouping the orbitals in two separate CAS $(4,4)$ active spaces over pairs of adjacent bonding/antibonding orbitals (i.e. 1,2,5,6 and 2,3,4,5) as in our ground-state scheme would miss the second most important single excitation in a CAS $(6,6)$ expansion, namely, the $3 \rightarrow 6$ transition. Furthermore, a similar analysis of the wave function for larger models of the retinal chromophore (with one, two, and three additional double bonds, namely, PSB4, PSB5, and PSB6) indicate that this behavior will persist and that far-away orbitals will remain correlated even though to a lesser extent. This is clear evidence that, for this problem, a large active space cannot be divided into several smaller active spaces on the basis of spatial vicinity of the orbitals but a different procedure must be followed to construct our wave function.

If we select the singles to build the domains so that all active orbitals are involved in at least one transition, we find that there are 6 important singles out of the reference (corresponding to a maximum weight of $2.19 \%$ in the excited state) and, correspondingly, three domains:

a) $\{1: 6\}$

b) $\{2: 5,6\}$

c) $\{3: 4,5,6\}$

For each domain, the number before the colon represents the doubly-occupied orbital in the reference which is linked via a single excitation to an empty orbital appearing after the colon. As already mentioned, the purpose of establishing the domains is to construct doubles and higher excitations by coupling the domains and to achieve in this way a compact form of the wave function. Therefore, while all domains can in principle be employed in generating higher transitions, we note that the most important singles belong to the $\mathbf{b}$ and $\mathbf{c}$ domains and choose to neglect the least important a domain, only retaining the singles within this domain and selected transitions as explained below.

We collect the configurations considered so far in the three excitation classes denoted as $\mathrm{X} 0, \mathrm{X} 1$, and $\mathrm{X} 2$, comprising the reference, the singles belonging to the dominant domains, and the important singles of the remaining domains, respectively. As next excitation class (X3), we include in our wave functions all double excitations 
Table 3.1: PSB3: CASSCF weight percentage of configurations with localized Res-1 orbitals computed at the S0 B3LYP equilibrium geometry.

\begin{tabular}{|c|c|c|c|c|c|c|c|}
\hline & \multirow[t]{2}{*}{ Type } & \multirow[t]{2}{*}{ Domain } & \multirow{2}{*}{$\begin{array}{l}\mathrm{CSF} \\
(\mathrm{N})\end{array}$} & \multirow{2}{*}{$\begin{array}{l}\text { Det } \\
(\mathrm{N})\end{array}$} & \multirow{2}{*}{$\begin{array}{c}\text { Configuration } \\
(1,2,3,4,5,6)\end{array}$} & \multicolumn{2}{|c|}{ Weight $(\%)$} \\
\hline & & & & & & S0 & S1 \\
\hline $\mathrm{X} 0$ & ref & & 1 & 1 & 222000 & 89.50 & 0.16 \\
\hline \multirow[t]{5}{*}{$\mathrm{X} 1$} & S & $\mathbf{b}$ & 1 & 2 & 212010 & 0.01 & 13.43 \\
\hline & S & b & 1 & 2 & 212001 & 0.46 & 18.60 \\
\hline & S & c & 1 & 2 & 221100 & 0.02 & 3.05 \\
\hline & $S$ & c & 1 & 2 & 221010 & 0.24 & 27.26 \\
\hline & S & c & 1 & 2 & 221001 & 0.26 & 21.03 \\
\hline Sub & & & 5 & 10 & & 0.99 & 83.37 \\
\hline $\mathrm{X} 2$ & $S$ & $\mathbf{a}$ & 1 & 2 & 122001 & 0.00 & 2.19 \\
\hline \multirow[t]{3}{*}{$\mathrm{X} 3$} & $\mathrm{D}$ & $\mathbf{a}$ & 1 & 1 & 022002 & 1.11 & 0.76 \\
\hline & $\mathrm{D}$ & b & 1 & 1 & 202020 & 2.31 & 2.75 \\
\hline & $\mathrm{D}$ & c & 1 & 1 & 220200 & 3.65 & 0.44 \\
\hline Sub & & & 3 & 3 & & 7.07 & 3.95 \\
\hline \multirow[t]{2}{*}{$\mathrm{X} 4$} & $\mathrm{D}$ & b & 1 & 1 & 202002 & 0.27 & 0.83 \\
\hline & $\mathrm{D}$ & c & 1 & 1 & 220020 & 0.10 & 0.62 \\
\hline Sub & & & 2 & 2 & & 0.37 & 1.45 \\
\hline \multirow[t]{3}{*}{$\mathrm{X} 5$} & $\mathrm{D}$ & $\mathbf{b}+\mathbf{c}$ & 2 & 6 & 211110 & 0.45 & 0.08 \\
\hline & $\mathrm{D}$ & $\mathbf{b}+\mathbf{c}$ & 2 & 6 & 211101 & 0.03 & 0.73 \\
\hline & $\mathrm{D}$ & $\mathbf{b}+\mathbf{c}$ & 2 & 6 & 211011 & 0.08 & 1.39 \\
\hline Sub & & & 6 & 18 & & 0.56 & 2.20 \\
\hline \multirow[t]{2}{*}{ X6 } & $\mathrm{D}$ & $\mathbf{b}+\mathbf{c}$ & 1 & 2 & 211020 & 0.01 & 0.57 \\
\hline & $\mathrm{D}$ & $\mathbf{b}+\mathbf{c}$ & 1 & 2 & 211002 & 0.05 & 0.51 \\
\hline Sub & & & 2 & 4 & & 0.06 & 1.08 \\
\hline \multirow[t]{4}{*}{$\mathrm{X} 7$} & $\mathrm{D}$ & b & 1 & 2 & 202011 & 0.01 & 0.04 \\
\hline & $\mathrm{D}$ & c & 1 & 2 & 220110 & 0.21 & 0.24 \\
\hline & $\mathrm{D}$ & c & 1 & 2 & 220101 & 0.02 & 0.26 \\
\hline & $\mathrm{D}$ & c & 1 & 2 & 220011 & 0.01 & 0.32 \\
\hline Sub & & & 4 & 8 & & 0.25 & 0.86 \\
\hline \multirow[t]{7}{*}{ X8 } & $\mathrm{T}$ & $\mathbf{b}+\mathrm{X} 3$ & 1 & 2 & 210210 & 0.00 & 0.34 \\
\hline & $\mathrm{T}$ & $\mathbf{b}+\mathrm{X} 3$ & 1 & 2 & 210201 & 0.01 & 0.46 \\
\hline & $\mathrm{T}$ & $\mathbf{b}+\mathrm{X} 3$ & 1 & 2 & 012012 & 0.00 & 0.08 \\
\hline & $\mathrm{T}$ & $\mathbf{c}+\mathrm{X} 3$ & 1 & 2 & 201021 & 0.00 & 0.53 \\
\hline & $\mathrm{T}$ & $\mathbf{c}+\mathrm{X} 3$ & 1 & 2 & 201120 & 0.00 & 0.01 \\
\hline & $\mathrm{T}$ & $\mathbf{c}+\mathrm{X} 3$ & 1 & 2 & 021012 & 0.00 & 0.11 \\
\hline & $\mathrm{T}$ & $\mathbf{c}+\mathrm{X} 3$ & 1 & 2 & 021102 & 0.00 & 0.03 \\
\hline Sub & & & 7 & 14 & & 0.01 & 1.56 \\
\hline Total & & & 31 & 62 & & 98.92 & 98.03 \\
\hline
\end{tabular}


from a bonding to its corresponding antibonding orbital, namely, $(1 \rightrightarrows 6),(2 \rightrightarrows 5)$, and $(3 \rightrightarrows 4)$. The importance of these excitations for the ground state was highlighted in our work on the development of a local correlation scheme for ground states [28] and is here corroborated in the PSB3 system: With the inclusion of the X3 class, the configurations make up $97.56 \%$ of the total ground-state CASSCF wave function. On the other hand, the X0-X3 classes only account for $89.67 \%$ of the total weight in the excited state.

To further improve the quality of the wave functions (in this case, the excitedstate one), we need to include additional double configurations, which we generate by coupling the single excitations within and between the dominant $\mathbf{b}$ and $\mathbf{c}$ domains. The transitions are then classified based on the orbital occupations and the resulting new excitation classes ordered according to their importance in the multi-determinant expansion. For instance, by coupling the single excitations within the c domain $\{3$ : $4,5,6\}$, we obtain

$$
\begin{aligned}
& 220200=(3 \rightrightarrows 4) \\
& 220020=(3 \rightrightarrows 5) \\
& 220002=(3 \rightrightarrows 6) \\
& 220110=(3 \rightarrow 4)+(3 \rightarrow 5) \\
& 220101=(3 \rightarrow 4)+(3 \rightarrow 6) \\
& 220011=(3 \rightarrow 5)+(3 \rightarrow 6)
\end{aligned}
$$

where $(3 \rightrightarrows 4)$ was already considered as a member of the $\mathrm{X} 3$ class. Configurations which are double excitations from a bonding to an adjacent antibonding orbitals are classified as X4 excitations, where orbitals are deemed adjacent only if located close to each other and not separated by an atom. Doubles from a bonding to a separated antibonding orbital, e.g. $(3 \rightrightarrows 6)$, are found to have negligible weight and are therefore not included in the X4 class. Double excitations from one bonding to two different antibonding orbitals form the X7 excitation class.

From the coupling of single excitations between the two $\mathbf{b}$ and $\mathbf{c}$ domains, we obtain the following doubles

$$
\begin{aligned}
& 211110=(2 \rightarrow 5)+(3 \rightarrow 4) \\
& 211020=(2 \rightarrow 5)+(3 \rightarrow 5) \\
& 211011=(2 \rightarrow 5)+(3 \rightarrow 6) \\
& 211101=(2 \rightarrow 6)+(3 \rightarrow 4) \\
& 211002=(2 \rightarrow 6)+(3 \rightarrow 6)
\end{aligned}
$$

Doubles with 4 unpaired electrons are classified as X5 and doubles from two bonding orbitals to the same antibonding orbital are denoted as X6, which exhausts the possible doubles one can build within one or two domains. We note that the ordering in importance of the X4-X7 doubles can be different for other states or molecules but, in this work, we will use the aforementioned ordering. The next excitation class (X8) is given by the triples constructed as the double excitations from a bonding to its corresponding antibonding orbital combined with singles from the X1 class. 
One can of course continue to construct further excitation classes and also couple more than two domains until all configurations in the CAS $(6,6)$ active space are included. Here, we however stop at X8 since the $\mathrm{X} 0-\mathrm{X} 8$ excitation classes cover $\sim 98 \%$ of the weight of both S0 and S1 states at the equilibrium geometry. The stateaveraged CASSCF weights of all configurations in the excitation class of PSB3 with the Res-1 orbitals are listed in Table 3.1 and the description of excitation classes is summarized in Table 3.2.

Table 3.2: Description of excitation classes. The X4-X7 excitations are built from the domains.

\begin{tabular}{cl}
\hline Class & Description \\
\hline X0 & References (all doubly-occupied bonding) \\
X1 & Important singles in dominant domains to build the doubles \\
X2 & Important singles in other domains \\
X3 & Bonding-antibonding doubles \\
X4 & Doubles to adjacent antibonding \\
X5 & Doubles (4 unpaired electrons) \\
X6 & Doubles (2 unpaired electron in bonding orbitals) \\
X7 & Doubles (2 unpaired electron in empty orbitals) \\
X8 & Triples (bonding-antibonding doubles plus X1 singles) \\
\hline
\end{tabular}

So far, our analysis demonstrates that the concept of orbital domains as identified at the CASSCF level leads to the construction of some of the most important excitation classes for both states. One may however ask whether these configurations remain important once we optimize the orbitals in the presence the Jastrow factor in a so-called Jastrow-Slater wave function:

$$
\Psi=\mathcal{J} \sum_{i}^{N_{\mathrm{CSF}}} c_{i} C_{i},
$$

where $C_{i}$ are the configuration state functions (CSF) and $\mathcal{J}$ is the Jastrow correlation function explicitly depending on the inter-electronic coordinates. In optimizing the orbitals, we do not preserve orthogonality among them, so the square of a CI coefficient in a Jastrow-Slater wave function is not the weight of a configuration. Therefore, we use the following definition of the weight of configurations in terms of the overlap $S_{i j}=\left\langle\mathcal{J} C_{i} \mid \mathcal{J} C_{j}\right\rangle$ :

$$
w_{i}=\frac{1}{q_{\mathrm{norm}}}\left(c_{i} S_{i i} c_{i}+\sum_{j \neq i}^{N_{\mathrm{CSF}}} c_{i} S_{i j} c_{j} z_{i j}\right),
$$

where the norm of the wave function is given by

$$
q_{\mathrm{norm}}=\sum_{i}^{N_{\mathrm{CSF}}} \sum_{j}^{N_{\mathrm{CSF}}} c_{i} S_{i j} c_{j},
$$


and, to reduce the occurrence of nonphysical negative weights, we introduced the factor

$$
z_{i j}=\frac{2\left(c_{i} S_{i i}\right)^{2}}{\left(c_{i} S_{i i}\right)^{2}+\left(c_{j} S_{j j}\right)^{2}}
$$

As shown in Table 3.3, the variational Monte Carlo (VMC) weights in a determinantal expansion over the Res-1 orbitals of PSB3 display a very good correspondence with the CASSCF weights. This ensures that the analysis of the excitation classes we have done above based on the CASSCF weights is transferable to VMC for this system.

Table 3.3: PSB3: CASSCF and VMC weight percentage of the X0-X3 configurations of the Res-1 structure computed at the S0 B3LYP equilibrium geometry. The VMC wave function includes all singles, doubles, and triples on the Res-1 local orbitals.

\begin{tabular}{ccrrrrr}
\hline Class & Configuration & \multicolumn{2}{c}{ CASSCF (\%) } & & \multicolumn{2}{c}{ VMC (\%) } \\
\cline { 3 - 4 } \cline { 7 - 8 } & $(1,2,3,4,5,6)$ & S0 & S1 & & S0 & S1 \\
\hline X0 & 222000 & 89.50 & 0.16 & & 92.85 & 2.41 \\
\hline X1 & 221100 & 0.02 & 3.05 & & 0.01 & 3.97 \\
& 221010 & 0.24 & 27.26 & & 0.97 & 33.06 \\
& 221001 & 0.26 & 21.03 & & 0.91 & 20.48 \\
& 212010 & 0.01 & 13.43 & & 0.10 & 12.77 \\
& 212001 & 0.46 & 18.6 & & 1.62 & 17.96 \\
\hline X2 & 122001 & 0.00 & 2.19 & & 0.00 & 1.15 \\
\hline X3 & 220200 & 3.65 & 0.44 & & 1.25 & 0.35 \\
& 202020 & 2.31 & 2.75 & & 0.66 & 1.71 \\
& 022002 & 1.11 & 0.76 & & 0.33 & 0.34 \\
\hline
\end{tabular}

\subsubsection{Beyond one resonance structure}

The importance of each resonance structure representation is directly related to the internuclear distances of the molecular structure under study. Therefore, while all orbital representations of Figure 3.1 are equivalent if we retain the complete expansion, we can no longer rely on the use of a single resonance structure once the localized CAS expansion is truncated based on excitation classes. For the PSB3 example, this means that, if one adopts the Res-1 picture above at a different geometry, there is no guarantee that the resulting excitations sum up to a similar, balanced weight percentage of the total wave functions as in Table 3.1. For instance, at a bond-inverted geometry which is more naturally described in terms of the Res- 2 orbitals, the expansions constructed from the X0-X8 excitation classes over CASSCF orbitals of the Res- 1 type only recover $\sim 98 \%$ and $\sim 74 \%$ of the total weights of the ground- and excited-state CASSCF wave functions, respectively. 
In general, one might expect that all three resonance structures will be "visited" in different regions of the excited-state potential energy surface of a molecule and that, to strive a balanced description of such diverse regions and account for changes in the relative importance of the three forms, our wave function should include all three of them. We can achieve this by taking advantage of the flexibility of the QMC method which allows us to work with an over-complete orbital set and write the Jastrow-Slater wave function as a linear combination of multiple Lewis structures, each expressed on a small set of determinants constructed according to the concepts of domains and excitation classes. Therefore, we rewrite the wave function in terms of all possible resonance structures of a molecule as

$$
\Psi=\mathcal{J}\left(\sum_{i} c_{i} C_{i}^{\mathrm{Res}-1}+\sum_{j} c_{j} C_{j}^{\mathrm{Res}-2}+\cdots\right),
$$

where $C_{i}^{\text {Res-I }}$ are the CSFs of the I-th resonance structure.

In constructing this multi-resonance wave function, it is desirable to adopt the same recipe for all resonance structures in the definition of the domains and the subsequent construction of the excitation classes. In the example of PSB3, the extension of the concept of orbital domains developed in the context of the Res- 1 structure to Res- 2 and Res-3 presents however a complication: In these two structures, the six $\pi$ electrons see four bonding (labeled 1, 2, 3, 4) and two antibonding (labeled 5, 6) orbitals (see panels C and D in Figure 3.1). Therefore, bonding orbitals are not necessarily doubly-occupied in the reference and this imbalance is reflected in the absence of a dominant reference configuration. We can nevertheless proceed as before if we consider each reference in turn, and define a corresponding set of domains from which to construct the doubles and other excitations. The union of the resulting excitations will then form our multi-resonance wave function. To analyze whether this way of extending the concept of domains to Res-2 and Res-3 is successful in recovering a high percentage of the total weights of the CASSCF wave functions, it is more appropriate to use a geometry where its internuclear distances resemble the bonding-antibonding structure of the resonance under study.

For the Res-2 structure, we therefore investigate the excitation classes using an in-plane bond-inverted geometry where we expect a description in terms of the Res-2 orbital set to be more suitable. By placing 6 electrons in 4 bonding orbitals, we can construct 4 doubly-occupied reference configurations: The important references are 222000, 220200, and 202200 and are included in the X0 class, while 022200 can be neglected since its weight is $0 \%$ in both states. From each reference, we then search for all important single excitations to build the domains. Here, a single excitation from a particular reference is a one-electron transition from a doubly-occupied bonding orbital to an empty (bonding or antibonding) orbital. Furthermore, one such transition may be obtained starting from more than one reference and therefore appear more than once as belonging to domains of different references. As in the case of Res-1, to decide which singles are important, we apply as threshold the maximum weight in either state so that all orbitals in the active space are involved in a single excitation (which is here $0.72 \%$ in the ground state). This criterion results in 9 impor- 
tant singles and three sets for domains as listed in Table 3.4. Using the same notation as for Res-1, the domains of the 222000 reference are :
1a) $\{1: 4\}$
1b) $\{2: 4\}$
1c) $\{3: 4\}$

The domains of 220200 are

2a) $\{1: 3\}$

2b) $\{2: 3,5\}$

2c) $\{4: 3\}$

and, for the 202200 reference, we have

3a) $\{1: 2\}$

3b) $\{3: 2,6\}$

3c) $\{4: 2,6\}$

These singles are included in the X1 class except for the least important $(2 \rightarrow 5)$ excitation of domain $\mathbf{2 b}$, which is included in the X2 class but further neglected in constructing the doubles to avoid many determinants with negligible weight on both states. Subsequent excitation classes are built and ordered according to the classification in the Res-1 structure as outlined in Table 3.4.

An analysis of the weights reveals that, also for this resonance structure, constructing the excitations based on the orbital domains and including the X0-X8 classes lead to a balanced description of the ground- and excited-state wave functions, recovering a substantial percentage of the total weight at the CASSCF level as shown in Table 3.5. For the third resonance structure (Res-3), we perform the same type of analysis at a geometry constructed to have a bond-length pattern and CASSCF gap in between the values of the ground-state equilibrium and bond-inverted geometries. Also for Res-2, the weights of the $\mathrm{X} 0-\mathrm{X} 8$ classes recover a very similar percentage of the ground- and excited-state CASSCF wave functions.

Therefore, the set of rules we have developed based on the concept of domains satisfy the prerequisite to be applicable without adjustments to the rather different resonance structures of PSB3. In practice, the analysis of the resonance structures at the different geometries is only performed to establish the domains for the different resonances and construct the dominant configurations. Then, for the geometry of interest, different sets of equivalent local orbitals are generated and the CSFs we have identified as important are used in a Jastrow-Slater wave function. Below, using PSB3 and other retinal models, we will employ one- and multi-resonance Jastrow-Slater wave functions to assess the performance of the approach, namely, to investigate numerically which excitation classes mainly impact the quality of a calculation, the accuracy of a one- and multiple-resonance wave function with respect to the use of truncated expansion over delocalized orbitals, and the transferability of this framework to geometries characteristic of different parts of the excited-state potential energy surface. 
Table 3.4: PSB3: Construction of the excitation classes of the Res-2 structure. Excitations in square brackets correspond to configurations already obtained.

\begin{tabular}{|c|c|c|c|}
\hline \multirow[t]{2}{*}{ Class } & \multicolumn{3}{|c|}{ Reference } \\
\hline & 222000 & 220200 & 202200 \\
\hline \multirow[t]{3}{*}{$\mathrm{X} 1$} & $1 \rightarrow 4$ & $1 \rightarrow 3$ & $1 \rightarrow 2$ \\
\hline & $2 \rightarrow 4$ & $2 \rightarrow 3$ & $3 \rightarrow[2], 6$ \\
\hline & $3 \rightarrow 4$ & {$[4 \rightarrow 3]$} & $4 \rightarrow[2], 6$ \\
\hline $\mathrm{X} 2$ & - & $2 \rightarrow 5$ & - \\
\hline \multirow[t]{2}{*}{$\mathrm{X} 3$} & $3 \rightrightarrows 5$ & $2 \rightrightarrows 6$ & $3 \rightrightarrows 5$ \\
\hline & $2 \rightrightarrows 6$ & & \\
\hline $\mathrm{X} 4$ & - & - & {$[3 \rightrightarrows 6]$} \\
\hline \multirow[t]{3}{*}{ X5 } & - & - & $1 \rightarrow 2+3 \rightarrow 6$ \\
\hline & & & $1 \rightarrow 2+4 \rightarrow 6$ \\
\hline & & & $3 \rightarrow 2+4 \rightarrow 6$ \\
\hline X6 & - & - & $3 \rightarrow 6+4 \rightarrow 6$ \\
\hline \multirow[t]{4}{*}{$\mathrm{X} 8$} & $1 \rightarrow 4+3 \rightrightarrows 5$ & $1 \rightarrow 3+2 \rightrightarrows 6$ & $1 \rightarrow 2+3 \rightrightarrows 5$ \\
\hline & $1 \rightarrow 4+2 \rightrightarrows 6$ & {$[4 \rightarrow 3+2 \rightrightarrows 6]$} & {$[4 \rightarrow 2+3 \rightrightarrows 5]$} \\
\hline & $2 \rightarrow 4+3 \rightrightarrows 5$ & & $4 \rightarrow 6+3 \rightrightarrows 5$ \\
\hline & {$[3 \rightarrow 4+2 \rightrightarrows 6]$} & & \\
\hline
\end{tabular}

Table 3.5: PSB3: CASSCF weight percentage of the excitation classes of the Res-2 structure. The in-plane bond-inverted S1 CASSCF geometry is used.

\begin{tabular}{cccrr}
\hline & CSF (N) & Det $(\mathrm{N})$ & \multicolumn{2}{c}{ Weight $(\%)$} \\
\cline { 3 - 5 } & & & S0 & S1 \\
\hline X0 & 3 & 3 & 12.60 & 23.29 \\
X1 & 8 & 16 & 76.92 & 64.64 \\
X2 & 1 & 2 & 0.72 & 0.43 \\
X3 & 4 & 4 & 0.42 & 0.94 \\
X5 & 6 & 18 & 2.66 & 2.84 \\
X6 & 1 & 2 & 0.33 & 1.07 \\
X8 & 6 & 12 & 0.66 & 1.43 \\
\hline Total & $\mathbf{2 9}$ & $\mathbf{5 7}$ & $\mathbf{9 3 . 5 9}$ & $\mathbf{9 4 . 2 1}$ \\
\hline
\end{tabular}

\subsubsection{Orbital optimization}

In the optimization of the orbitals of a monodeterminant Jastrow-Slater wave function, one mixes the occupied orbitals with the virtual ones of the same symmetry. In the multideterminantal case, the inactive orbitals are mixed with the active and virtual ones, and the active orbitals with other active and the virtual orbitals, always accord- 
ing to the symmetry. For large systems, the number of orbital mixing grows rapidly and, to maintain a low computational cost, one is often forced to select a subset of virtual orbitals to mix with the occupied ones: The delocalized virtual orbitals are for instance ordered energetically and the highest-energy virtual orbitals are omitted.

If we unitarily transform all (inactive, active, and virtual) delocalized orbitals into localized ones, we can employ a different criterion and selectively mix the orbitals based on spatial considerations. First, to establish the centers where an orbital is mainly localized, we use its expansion over the $N_{\text {bas }}$ atomic basis functions $\chi_{i}$ as $\sum_{i} b_{i} \chi_{i}$ and evaluate the quantities $\rho_{a}$ for all nuclei:

$$
\rho_{a}=2 \sum_{i}^{M_{a}} \sum_{j}^{N_{\text {bas }}} b_{i}^{*} b_{j}\left\langle\chi_{i} \mid \chi_{j}\right\rangle,
$$

where the first summation only runs over the $M_{a}$ atomic basis functions of nucleus $a$. A large value of $\rho_{a}$ indicates that the orbital is mainly localized on atom $a$ and one can use a threshold on the value of $\rho_{a}$ to determine the centers contributing to the decomposition of the orbital. Then, an orbital is mixed with another orbitals if they share at least one nuclear center according to the given threshold, which we name below "threshold on orbital mixing" (TM).

We test several values of TM in the VMC wave function optimization of the PSB2, PSB3, and PSB4 and list the results in Table 3.6. We find that both groundand excited-state energies are of course higher when we apply this threshold since we loose some degree of variationality in the optimization. On the other hand, the excitation energy remains unchanged and the computational saving is significant being as large as a factor of 6 in the number of orbital parameters to optimize for the largest PSB4. Therefore, this trick to cut the computational cost in the orbital optimization step is effective and allows one to treat both states on an equal footing.

\subsection{Computational details}

The QMC calculations are performed with the CHAMP program [33] with scalarrelativistic energy-consistent Hartree-Fock pseudopotentials and the corresponding basis sets $[34,35]$. In particular, we use the double (D), triple $\left(\mathrm{T}^{\prime}\right)$ and quadruple $\left(\mathrm{Q}^{\prime}\right)$ basis sets, where the prime denotes that a double (D) basis is used for the hydrogen atoms, and the aug-cc-pVDZ' (D+) basis set which is a double basis set augmented with additional $s$ and $p$ functions on the heavy atoms [36]. To account for electronelectron and electron-nucleus correlations, we use a two-body Jastrow factor which is different for every atom types [37]. The Jastrow parameters, CI coefficients, and orbital coefficients are optimized with the linear method [38] in a state-averaged fashion [39] within VMC. In the diffusion Monte Carlo (DMC) calculations, we treat the non-local pseudopotentials beyond the locality approximation [40] and use an imaginary time-step of 0.01 a.u.

To determine the initial inactive localized orbitals for the VMC trial wave function, several localization techniques [41-44] are possible, which unitarily transform 
Table 3.6: VMC total and excitation energies of the PSBn models computed with the Res-1 localized orbitals and different thresholds on the orbital mixing in the wave function optimization. The S0 B3LYP geometry is used.

\begin{tabular}{lcccc}
\hline Molecule & $\begin{array}{c}\text { Thr. mixing } \\
\text { (parms optimized) }\end{array}$ & $\begin{array}{c}\text { S0 } \\
\text { (a.u.) }\end{array}$ & $\begin{array}{c}\text { S1 } \\
\text { (a.u.) }\end{array}$ & $\begin{array}{c}\Delta \mathrm{E} \\
(\mathrm{eV})\end{array}$ \\
\hline PSB2 $^{*}$ & $0.0(579)$ & $-30.2664(3)$ & $-30.0458(3)$ & $6.00(1)$ \\
& $0.1(190)$ & $-30.2626(3)$ & $-30.0416(3)$ & $6.01(1)$ \\
& $0.2(170)$ & $-30.2614(3)$ & $-30.0408(3)$ & $6.00(1)$ \\
\hline PSB3 $^{\dagger}$ & $0.00(1213)$ & $-42.8046(5)$ & $-42.6441(5)$ & $4.37(2)$ \\
& $0.05(309)$ & $-42.7989(5)$ & $-42.6382(5)$ & $4.37(2)$ \\
& $0.10(290)$ & $-42.7977(5)$ & $-42.6372(5)$ & $4.37(2)$ \\
& $0.20(270)$ & $-42.7974(5)$ & $-42.6359(5)$ & $4.39(2)$ \\
& $0.30(257)$ & $-42.7963(5)$ & $-42.6357(5)$ & $4.37(2)$ \\
\hline PSB4 $^{\ddagger}$ & $0.00(2079)$ & $-55.3447(5)$ & $-55.2125(5)$ & $3.60(2)$ \\
& $0.10(390)$ & $-55.3359(5)$ & $-55.2035(5)$ & $3.60(2)$ \\
& $0.20(364)$ & $-55.3348(5)$ & $-55.2017(5)$ & $3.62(2)$ \\
& $0.30(349)$ & $-55.3338(5)$ & $-55.2010(5)$ & $3.61(2)$ \\
\hline
\end{tabular}

${ }^{*}$ All CAS $(4,4)$ determinants are included.

${ }^{\dagger}$ All singles, doubles, and triples in the CAS $(6,6)$.

$\ddagger$ CSFs with $\left|c_{i}\right|^{2}>0.011$ ( $\sim 99 \%$ of CASSCF weight of both states

the delocalized CASSCF orbitals into 1-or-2-centered localized orbitals. Here, we employ the Pipek-Mezey method [41] to localize the inactive and the virtual orbitals using the Molcas-7.8 [45] package. For the active space, we are interested in constructing localized orbitals that are mainly located on the atom/bond associated with a particular Lewis resonance structure. To this aim, we construct an initial guess of active orbitals with the desired structure, whose shape we find to be preserved in the subsequent CASSCF calculation. For the Res- 1 structure, the initial localized guess are obtained in a multi-configuration self-consistent-field calculation including the reference and a set of bonding-antibonding double excitations using the GAMESS(US) code $[46,47]$. The initial localized active orbitals for the other resonance structures are prepared manually.

Upon optimization in VMC, we find that the active orbital might become delocalized and this undermines the construction of the multi-determinant expansion based on the concept of domains and excitation classes. We find that, to ensure the locality of active orbitals, it is sufficient to limit the mixing of the active orbitals among themselves: A bonding orbital is only mixed with its adjacent bonding orbitals and the antibonding orbitals that share at least one nucleus; an antibonding orbital is only mixed with a bonding orbital that shares one nucleus but not with other antibonding ones.

We perform excited-state geometry optimizations of the PSB3 at the multi-state complete-active-space second-order perturbation theory (MS-CASPT2) level [48- 
50] using the Molcas-7.8 package. We use the Cholesky decomposition of the twoelectron integrals [51] with a threshold of $10^{-4}$ and always adopt the default 0.25 IPEA zero-order Hamiltonian [52]. Finally, we optimize the ground-state geometry of the PSBn chromophores within DFT with the B3LYP functional [53-55] and the cc-pVDZ basis set using the Gaussian code [56] and the S1 planar bond-inverted CASSCF geometries with the cc-pVDZ basis and the Molcas-7.8 package.

\subsection{Results and discussions}

We test our novel form of Jastrow-Slater wave functions for excited states using the retinal protonated Schiff base model chromophores depicted in Figure 3.2. The smallest PSB2 model has two conjugated $\pi$ bonds and by adding one and two more ethylenic groups, one obtains the PSB3 and PSB4 chromophores.

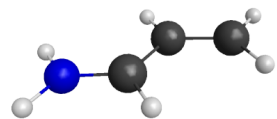

PSB2

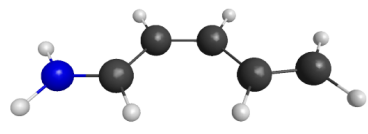

PSB3

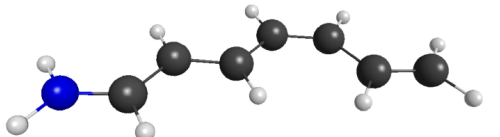

PSB4

Figure 3.2: Molecules studied in this work. Blue, black, and grey represent nitrogen, carbon, and hydrogen, respectively.

To assess the flexibility of the domain and multi-resonance concepts, we compute the VMC vertical excitation energies of the $\pi \rightarrow \pi^{*}$ state of the PSBn molecules at two in-plane geometries with different bond-length patterns and compare them with the reference values obtained using a complete (or nearly complete) expansion over the active orbitals (the configurations in the reference cover at least $\sim 99 \%$ of the CASSCF weights in the ground and excited states). The two test geometries are characterized by a positive and a negative value of the bond-length alternation (BLA) which we define as the difference between the averages of formal single and double $\mathrm{C}-\mathrm{C}$ bonds, where "formal" refers to the ground-state geometry (or Res-1 Lewis structure). The two geometries are the ground-state B3LYP geometry and the bondinverted S1 CASSCF geometry, respectively. To test the performance of our method in the relaxation of excited states, we also perform the out-of-plane excited-state geometry optimization of PSB3 at the VMC level. Finally, we compute the DMC total and excitation energies for single and multi-resonance local wave functions at the ground-state geometry. 


\subsubsection{PSB2}

The minimal active space to describe the $\pi \rightarrow \pi^{*}$ excited state in PSB2 is a CAS $(4,4)$ expansion where we correlate 4 electrons in $4 \pi$ orbitals. The molecule has two resonance structures with corresponding localized active $\pi$ orbitals shown in Figure 3.3.

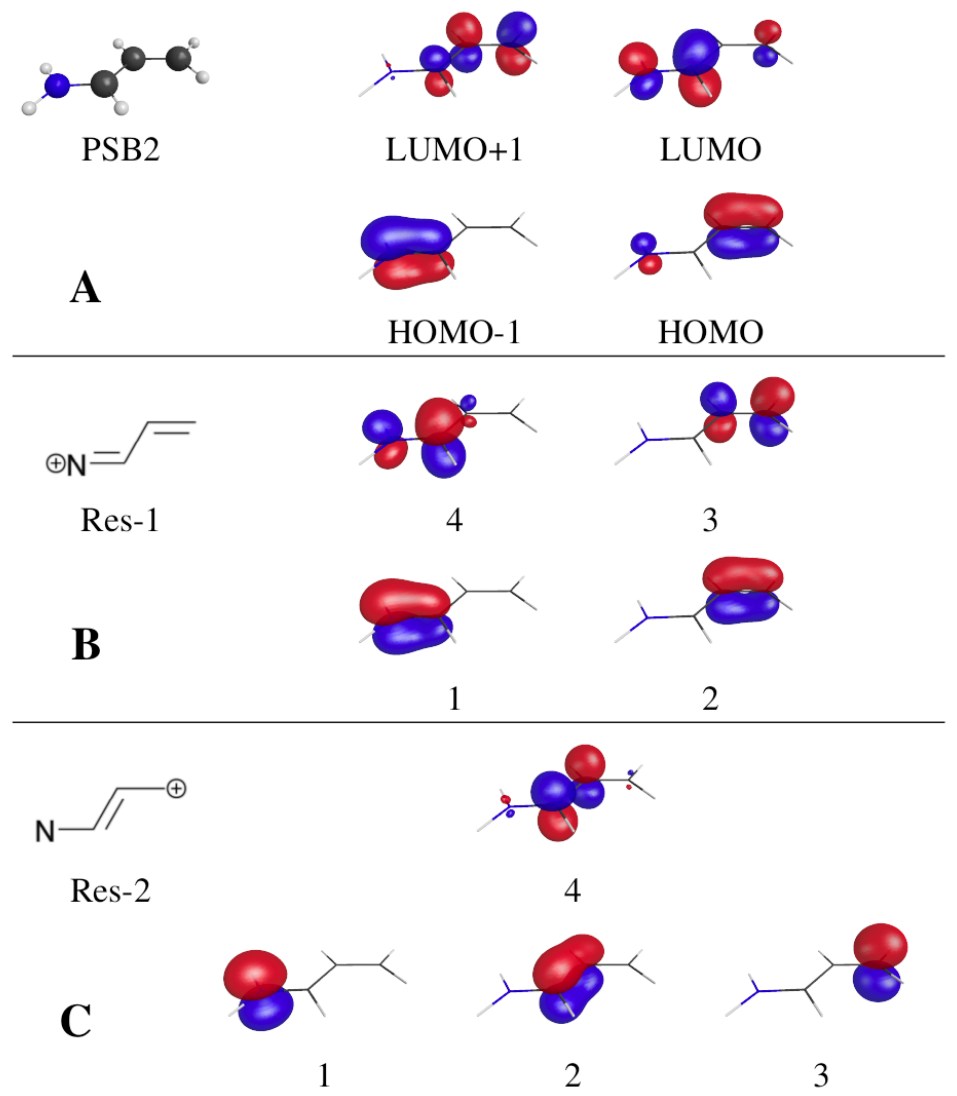

Figure 3.3: PSB2: (A) Active delocalized $\pi$ orbitals obtained at the CASSCF $(4,4)$ level, and $(\mathrm{B}, \mathrm{C})$ two unitary transformed sets of localized orbitals corresponding to the two Lewis resonance structures.

Before addressing the performance of the wave functions proposed here for PSB2, we investigate the effect of using different active spaces, number of determinants, and basis sets on the vertical excitation energy at the VMC level. In these tests, the determinantal expansion is expressed on the delocalized natural orbitals and all parameters of the wave function are optimized. In particular, all (inactive and active) orbitals are mixed with the active and virtual ones according to the symmetry. For the larger active spaces, the expansion is truncated according to a threshold on the CI coefficients on both states, and the union of the surviving configurations is retained in the Jastrow-Slater wave functions. The results are collected in Table 3.7.

We find that enlarging the active space beyond the minimal one with the inclusion of additional $\pi$ orbitals results in the decrease of the energies of both states but that the excitation energy remains compatible within the statistical error. The choice 
Table 3.7: PSB2: VMC energies computed with different basis sets and active spaces expressed on the weighted-averaged natural orbitals at the S0 B3LYP geometry.

\begin{tabular}{llcccc}
\hline $\begin{array}{l}\text { Active } \\
\text { Space }\end{array}$ & $\begin{array}{l}\text { Thr. } \\
(\mathrm{N} \mathrm{det})\end{array}$ & Basis & $\begin{array}{c}\text { S0 } \\
\text { (a.u.) }\end{array}$ & $\begin{array}{c}\text { S1 } \\
(\text { a.u. })\end{array}$ & $\begin{array}{c}\Delta \mathrm{E} \\
(\mathrm{eV})\end{array}$ \\
\hline CAS $(4,4)$ & $0.00(36)$ & $\mathrm{D}$ & $-30.2661(3)$ & $-30.0460(3)$ & $5.99(1)$ \\
CAS $(4,6)$ & $0.02(58)$ & $\mathrm{D}$ & $-30.2677(3)$ & $-30.0471(3)$ & $6.00(1)$ \\
CAS $(4,6)$ & $0.01(99)$ & $\mathrm{D}$ & $-30.2677(3)$ & $-30.0478(3)$ & $5.98(1)$ \\
CAS $(4,8)$ & $0.02(83)$ & $\mathrm{D}$ & $-30.2677(3)$ & $-30.0471(3)$ & $6.00(1)$ \\
CAS $(4,8)$ & $0.01(125)$ & $\mathrm{D}$ & $-30.2678(3)$ & $-30.0479(3)$ & $5.98(1)$ \\
CAS $(4,4)$ & $0.00(36)$ & $\mathrm{D}+$ & $-30.2673(3)$ & $-30.0501(3)$ & $5.91(1)$ \\
CAS $(4,4)$ & $0.00(36)$ & $\mathrm{T}^{\prime}$ & $-30.2782(3)$ & $-30.0602(3)$ & $5.93(1)$ \\
CAS $(4,4)$ & $0.00(36)$ & $\mathrm{Q}^{\prime}$ & $-30.2793(3)$ & $-30.0621(3)$ & $5.91(1)$ \\
\hline
\end{tabular}

of basis set has a larger impact on the excitation energy, which is however practically converged with the D+ basis set. For the purpose of assessing our excitedstate method, we select the CAS(2n,2n) active space and D basis set for the PSBn molecules as compromise between accuracy and computational cost, keeping in mind that, if one is interested in the best excitation energies of PSBn, one should use a larger basis set.

We now begin the construction of the local wave functions, whose performance is assessed against a VMC calculation that includes all (36) determinants of the CAS $(4,4)$ expansion. With this active space, the Res-1 localized orbitals consist of 2 bonding and 2 antibonding orbitals and the dominant doubly-occupied configurations (2200) is the reference configuration. In the CASSCF calculation, there are 3 important singles, which result in the domains:

a) $\{1: 4\}$

b) $\{2: 3,4\}$

As in the case of PSB3, the charge transfer character of the excited state is evident from the domains as the most important single $(2 \rightarrow 4)$ results in a net charge transfer from the $\mathrm{C}$-terminus to the $\mathrm{N}$-terminus. For the Res- 2 structure, we can place 4 electrons in 3 bonding and 1 antibonding orbitals and, among the three possible doublyoccupied configurations, retain 2 references for establishing the domains, namely, 2200 and 2020. The remaining doubly-occupied configuration (0220) is unimportant since its weight is almost $0 \%$ in both states. The domains of the 2200 reference are

1a) $\{1: 3,4\}$

1b) $\{2: 3\}$

while, for the 2020 reference, we have

2a) $\{1: 2\}$

The excitation classes are constructed and ordered in the same way as for PSB3 and the resulting configurational weights are listed in Table 3.8, for the Res-1 structure at the ground-state geometry and for Res- 2 at the bond-inverted geometry, respectively. 
Table 3.8: PSB2: CASSCF weight percentage of the excitation classes of the Res-1 and the Res-2 structure at the in-plane S0 B3LYP and the bond-inverted S1 CASSCF geometry, respectively.

\begin{tabular}{|c|c|c|c|c|c|c|}
\hline & \multicolumn{3}{|c|}{ Res-1 } & \multicolumn{3}{|c|}{ Res-2 } \\
\hline & \multirow[t]{2}{*}{$\operatorname{Det}(\mathrm{N})$} & \multicolumn{2}{|c|}{ Weight (\%) } & \multirow[t]{2}{*}{$\operatorname{Det}(\mathrm{N})$} & \multicolumn{2}{|c|}{ Weight (\%) } \\
\hline & & S0 & S1 & & S0 & S1 \\
\hline X0 & 1 & 93.60 & 0.05 & 2 & 18.13 & 16.40 \\
\hline $\mathrm{X} 1$ & 6 & 0.63 & 91.67 & 8 & 75.37 & 74.78 \\
\hline X3 & 2 & 4.84 & 4.75 & 1 & 0.25 & 0.24 \\
\hline X4 & 1 & 0.24 & 2.24 & 1 & 0.01 & 0.08 \\
\hline X5 & 6 & 0.22 & 0.25 & 6 & 3.12 & 4.87 \\
\hline X6 & 2 & 0.00 & 0.06 & & & \\
\hline X7 & 2 & 0.21 & 0.00 & 2 & 0.14 & 0.41 \\
\hline X8 & 4 & 0.00 & 0.23 & 2 & 0.41 & 2.34 \\
\hline Total & 24 & 99.74 & 99.25 & 22 & 97.41 & 99.12 \\
\hline
\end{tabular}

Here, we want to understand the minimum set of ingredients in the local wave functions needed to yield the reference energies. Therefore, we include systematically the excitation classes of one and two resonance structures in the Jastrow-Slater trial wave functions of PSB2 and fully reoptimize each trial wave function in VMC. The resulting ground- and excited-state energies computed at the ground-state and bond-inverted geometries are reported in Table 3.9. At the equilibrium geometry, if we use a single Res- 1 structure, we find that the excitation energy is already converged with the inclusion of the $\mathrm{X} 0-\mathrm{X} 4$ excitation classes (10 determinants). On the other hand, the ground- and excited-state energies display a slower convergence, which is is only achieved when all X0-X8 transitions (24 determinants) are included.

If we employ a multi-resonance Jastrow-Slater wave function and account for both the Res-1 and Res- 2 resonances in the multi-determinantal wave function, the energies of both states are already in agreement with the reference values when we include the sole $\mathrm{X} 0-\mathrm{X} 3$ classes for each resonance. Consequently, we obtain a somewhat more compact (20 determinants) wave function for PSB2 when using two instead of a single resonance structure. If we perform a similar analysis on the bond-inverted geometry of PSB2, we obtain an equivalently good performance of the multi-resonance wave function expressed on the $\mathrm{X} 0-\mathrm{X} 3$ excitations of both resonance structures.

We note that, in the orbital optimization step, the active orbitals become occasionally delocalized and that the loss in locality is especially pronounced when moving to larger systems. Therefore, all results for PSB2 in Table 3.9 (as well as below for PSB3 and PSB4) are obtained with the restriction on the mixing among the active orbitals. This restriction is found to generally preserve locality but leads to an increase in the energies and is in part responsible for their slower convergence with the excitation classes. The effect of this restriction becomes however negligible once 
Table 3.9: PSB2: VMC total and excitation energies computed with an increasing number of excitation classes of one or two resonance structures at the S0 B3LYP $(\mathrm{BLA}>0)$ and S1 CASSCF $(\mathrm{BLA}<0)$ geometries.

\begin{tabular}{llccc}
\hline Resonance & Determinant (N) & $\begin{array}{c}\text { S0 } \\
(\text { a.u. })\end{array}$ & $\begin{array}{c}\text { S1 } \\
(\text { a.u. })\end{array}$ & $\begin{array}{c}\Delta \mathrm{E} \\
(\mathrm{eV})\end{array}$ \\
\hline BLA $>$ 0 geometry & & & \\
1 & X0-X1 (7) & $-*$ & $-*$ & $-*$ \\
1 & X0-X3 (9) & $-30.2638(3)$ & $-30.0403(3)$ & $6.08(1)$ \\
1 & X0-X4 (10) & $-30.2649(3)$ & $-30.0437(3)$ & $6.02(1)$ \\
1 & X0-X5 (16) & $-30.2651(3)$ & $-30.0444(3)$ & $6.01(1)$ \\
1 & X0-X6 (18) & $-30.2651(3)$ & $-30.0437(3)$ & $6.02(1)$ \\
1 & X0-X7 (20) & $-30.2652(3)$ & $-30.0442(3)$ & $6.01(1)$ \\
1 & X0-X8 (24) & $-30.2656(3)$ & $-30.0449(3)$ & $6.01(1)$ \\
1,2 & X0-X1 (17) & $-*$ & $-*$ & $-*$ \\
1,2 & X0-X3 (20) & $-30.2670(3)$ & $-30.0464(3)$ & $6.00(1)$ \\
$1,2^{\dagger}$ & X0-X3 (20) & $-30.2616(3)$ & $-30.0412(3)$ & $6.00(1)$ \\
1 & All (36) & $-30.2664(3)$ & $-30.0458(3)$ & $6.00(1)$ \\
\hline BLA $<$ 0 geometry & & & \\
1,2 & X0-X3 (20) & $-30.2176(3)$ & $-30.0512(3)$ & $4.53(1)$ \\
$1,2^{\dagger}$ & X0-X3 (20) & $-30.2128(3)$ & $-30.0455(3)$ & $4.55(1)$ \\
1 & All (36) & $-30.2175(3)$ & $-30.0513(3)$ & $4.52(1)$ \\
\hline
\end{tabular}

${ }^{*}$ The run results in delocalized optimized active orbitals.

$\dagger \mathrm{TM}=0.2$ on the occupied-virtual orbital mixing.

we include the $\mathrm{X} 0-\mathrm{X} 8$ excitation classes or multiple resonances in combination with fewer classes. In Table 3.9, we further test the effect of limiting the occupied-virtual orbital mixing in the orbital optimization of the multi-resonance wave function by applying a threshold $\mathrm{TM}=0.2$. We find that, at both geometries, the energies of the S0 and S1 states raise by about 4-5 mHartree but that the excitation energies remain compatible with the reference values.

\subsubsection{PSB3}

The construction of the excitation classes of the Res-1 and Res-2 structures of PSB3 is explained in detail in Section 3.2 whereas the construction for the Res-3 structure follows the same recipe as the Res-2. Having all excitation classes built, we can progressively add them in the wave function as shown in Table 3.10. The reference energies are computed as an expansion over all singles, doubles, and triples excitations expressed in terms of the Res- 1 orbitals, whose total weight at the SA-CASSCF level is more than $99 \%$ for both states. Note that we restrict the active orbital mixing in the orbital optimization as for PSB2 to guarantee their locality upon optimization.

Differently from the case of the PSB2, the convergence of the excitation energy and the total energies of both states is not achieved by including the $\mathrm{X} 0-\mathrm{X} 8$ exci- 
Table 3.10: PSB3: VMC total and excitation energies computed with an increasing number of excitation classes of one, two, and three resonance structures at the S0 B3LYP (BLA > 0) and S1 CASSCF (BLA < 0) geometries.

\begin{tabular}{|c|c|c|c|c|}
\hline Resonance & Determinant (N) & $\begin{array}{c}\text { S0 } \\
\text { (a.u.) }\end{array}$ & $\begin{array}{c}\text { S1 } \\
\text { (a.u.) }\end{array}$ & $\begin{array}{c}\Delta \mathrm{E} \\
(\mathrm{eV})\end{array}$ \\
\hline \multicolumn{5}{|c|}{ BLA $>0$ geometry } \\
\hline 1 & X0-X2 (13) & $-42.7865(5)$ & $-42.6150(5)$ & $4.67(2)$ \\
\hline 1 & X0-X3 (16) & $-42.7980(5)$ & $-42.6262(5)$ & $4.67(2)$ \\
\hline 1 & X0-X4 (18) & $-42.8002(5)$ & $-42.6292(5)$ & $4.65(2)$ \\
\hline 1 & X0-X5 (36) & $-42.7989(5)$ & $-42.6359(5)$ & $4.44(2)$ \\
\hline 1 & X0-X6 (40) & $-42.8012(5)$ & $-42.6369(5)$ & $4.47(2)$ \\
\hline 1 & X0-X7 (48) & $-42.8017(5)$ & $-42.6382(5)$ & $4.45(2)$ \\
\hline 1 & X0-X8 (56) & $-42.8015(5)$ & $-42.6392(5)$ & $4.42(2)$ \\
\hline 1,2 & X0-X3 (41) & $-42.8029(5)$ & $-42.6396(5)$ & $4.44(2)$ \\
\hline 1,2 & X0-X4 (43) & $-42.8032(5)$ & $-42.6406(5)$ & $4.42(2)$ \\
\hline 1,2 & X0-X5 (79) & $-42.8041(5)$ & $-42.6412(5)$ & $4.43(2)$ \\
\hline 1,2 & X0-X6 (85) & $-42.8037(8)$ & $-42.6403(8)$ & $4.45(3)$ \\
\hline 1,2 & X0-X7 (93) & $-42.8041(5)$ & $-42.6410(5)$ & $4.44(2)$ \\
\hline 1,2 & X0-X8 (119) & $-42.8039(5)$ & $-42.6396(5)$ & $4.47(2)$ \\
\hline $1,2,3$ & X0-X3 (66) & $-42.8045(5)$ & $-42.6431(5)$ & $4.39(2)$ \\
\hline $1,2,3^{\dagger}$ & X0-X3 (66) & $-42.7973(5)$ & $-42.6352(5)$ & $4.41(2)$ \\
\hline Reference $\ddagger$ & & $-42.8046(5)$ & $-42.6441(5)$ & $4.37(2)$ \\
\hline \multicolumn{5}{|c|}{ BLA $<0$ geometry } \\
\hline $1,2,3$ & X0-X3 (66) & $-42.7823(5)$ & $-42.6470(5)$ & $3.68(2)$ \\
\hline $1,2,3^{\dagger}$ & X0-X3 (66) & $-42.7740(5)$ & $-42.6385(5)$ & $3.69(2)$ \\
\hline Reference $\ddagger$ & & $-42.7823(5)$ & $-42.6468(5)$ & $3.69(2)$ \\
\hline
\end{tabular}

tation classes of one (Res-1) or two (Res-1 and Res-2) resonance structures. While the ground-state energy is converged with a wave function expressed on the X0-X5 transitions of the Res- 1 and Res- 2 structures (79 determinants), the excited-state energy needs more configurations to reach its reference value. We stress that the lack of convergence is here not due to the restriction on the mixing among the active orbitals in the wave function optimization. Therefore, one needs to construct and include additional excitation classes (X9, X10, etc.) if one keeps the expansion over one or two resonance structures.

If we include also the remaining resonance structure (Res-3) and add the corresponding excitation classes, we obtain that the convergence of the energy of both states and, consequently, of the excitation energy is reached by just including the $\mathrm{X} 0-\mathrm{X} 3$ excitation classes of all three resonance structures in the expansion. This wave function is compact and only contains 66 determinants. The same multiresonance expansion is employed for the bond-inverted geometry and is found to 
describe equally well both states and the excitation energy, which are in agreement with the reference values. We can further reduce the computational cost by applying $\mathrm{TM}=0.2$ in the optimization of the wave function which leads to an increase of the energies of both states by about 8-9 mHartree but to a compatible excitation energy.

\subsubsection{PSB4}

The largest retinal model investigated in this work is PSB4 which has four dominant Lewis resonance structures as depicted in Figure 3.4. At the ground-state equilibrium geometry, the wave function of the $\pi \rightarrow \pi^{*}$ excited state is dominated by the Res- 1 structure while the other resonances become more important in the relaxation process. The important singles, the domains, and the weights in the localized CASSCF wave functions are reported for Res-1 in the Appendix.
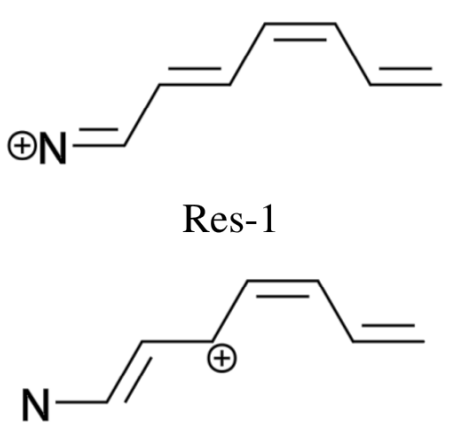

Res-3

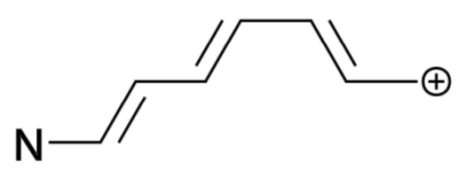

Res-2

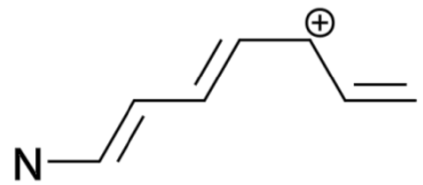

Res-4

Figure 3.4: PSB4: Dominant Lewis structures.

Table 3.11: PSB4: VMC total and excitation energies computed with a wave function including all resonance structures at the S0 B3LYP (BLA >0) and S1 CASSCF $(\mathrm{BLA}<0)$ geometries.

\begin{tabular}{lcccc}
\hline Resonance & Determinant (N) & $\begin{array}{c}\text { S0 } \\
\text { (a.u.) }\end{array}$ & $\begin{array}{c}\text { S1 } \\
(\text { a.u. })\end{array}$ & $\begin{array}{c}\Delta \mathrm{E} \\
(\mathrm{eV})\end{array}$ \\
\hline \multicolumn{1}{l}{ BLA $>$ 0 geometry } & & & \\
$1,2,3,4$ & $\mathrm{X} 0-\mathrm{X} 3(159)$ & $-55.3454(5)$ & $-55.2128(5)$ & $3.61(2)$ \\
$1,2,3,4^{*}$ & $\mathrm{X} 0-\mathrm{X} 3(159)$ & $-55.3346(5)$ & $-55.2011(5)$ & $3.63(2)$ \\
Reference $^{\dagger}$ & $(433)$ & $-55.3441(5)$ & $-55.2123(5)$ & $3.59(2)$ \\
\hline BLA $<\mathbf{0}$ geometry & & & & \\
$1,2,3,4$ & $\mathrm{X} 0-\mathrm{X} 3(159)$ & $-55.3237(5)$ & $-55.2149(5)$ & $2.96(2)$ \\
$1,2,3,4^{*}$ & $\mathrm{X} 0-\mathrm{X} 3(159)$ & $-55.3129(5)$ & $-55.2037(5)$ & $2.97(2)$ \\
Reference $^{\dagger}$ & $(485)$ & $-55.3238(5)$ & $-55.2160(5)$ & $2.93(2)$ \\
\hline
\end{tabular}

$* \mathrm{TM}=0.2$ on the occupied-virtual orbital mixing.

$\dagger \sim 99 \%$ of CASSCF weights on delocalized natural orbitals. 
Also for PSB4, we test the performance of the multi-resonance wave function that includes the $\mathrm{X} 0-\mathrm{X} 3$ excitation classes of all possible resonance structures and report the results in Table 3.11. To compute the reference energies, we construct Jastrow-Slater wave functions with determinantal expansions over delocalized natural orbitals, which recover at least $99 \%$ of the CASSCF weights of both states. At both the equilibrium ground-state and the bond-inverted in-plane geometry, the ground- and excited-state energies and the excitation energy of the multi-resonance wave functions are in very good agreement with the reference values. The resulting wave function is very compact with a number of determinants (159) which is lower by a factor of 2-3 at the two geometries than the one used to compute the reference energies. Further reduction of computational cost can be obtained by applying a $\mathrm{TM}=0.2$ in the orbital optimization step to limit the occupied-virtual orbital mixing (the number of parameter to optimize is reduced by almost a factor of 6 - see Table 3.6). As in the case of PSB2 and PSB3, the resulting energy gap remains compatible with the reference value.

\subsubsection{Geometry optimization}

The wave function expanded on the $\mathrm{X} 0-\mathrm{X} 3$ excitation classes of all possible resonance structures is a compact and accurate wave function for the computation of the vertical excitation energies of PSBn $(n=2,3,4)$ at two different in-plane geometries. We now test its ability to describe the excited-state relaxation by performing the out-of-plane geometry optimization of one retinal model chromophore, namely, the PSB3. To reduce the computational cost, we apply $\mathrm{TM}=0.2$ in the orbital optimization and compare the resulting optimized geometries against the ones obtained from a fully optimized Jastrow-Slater wave function whose determinantal expansion over delocalized natural orbitals is truncated to recover $99 \%$ of the total CASSCF weight of both states. We also compare the VMC excited-state geometries to the optimal CASPT2 ones.

The relevant optimized excited-state geometrical parameters are shown in Table 3.12. The atoms are numbered and the covalent bonds ordered as $N_{1}=C_{1}-C_{2}=C_{3}-$ $\mathrm{C}_{4}=\mathrm{C}_{5}$. We find that the optimized geometrical parameters obtained with the local multi-resonance wave function differ by less than $3 \mathrm{~m} \AA$ and $1^{\circ}$ from the VMC reference values computed with the same $\mathrm{D}$ basis set. This demonstrates the good performance of our localized representation of the wave function also in the optimization of the excited-state geometry. Finally, to compare the VMC and MS-CASPT2 geometries, we employ the triple- and quadruple-zeta (double-zeta for hydrogens) basis sets, respectively, since VMC benefits from a somewhat faster basis-set convergence of the structural parameters and observe an excellent agreement between the equilibrium structures obtained with the two methods with differences smaller than $7 \mathrm{~m} \AA$ and $2^{\circ}$. 
Table 3.12: PSB3: VMC out-of-plane optimized geometries.

\begin{tabular}{|c|c|c|c|c|}
\hline \multirow{3}{*}{$\begin{array}{l}\text { Method } \\
\text { Basis set }\end{array}$} & \multicolumn{3}{|c|}{ VMC } & \multirow[t]{2}{*}{ CASPT2 $(6,6)$} \\
\hline & Reference* & Local $^{\dagger}$ & Local $^{\dagger}$ & \\
\hline & $\mathrm{D}$ & D & $\mathrm{T}^{\prime}$ & cc-pVQZ' \\
\hline $\mathrm{N}_{1}=\mathrm{C}_{1}$ & $1.3800(2)$ & $1.3801(7)$ & $1.3738(4)$ & 1.3740 \\
\hline $\mathrm{C}_{1}-\mathrm{C}_{2}$ & $1.4674(1)$ & $1.4666(14)$ & $1.4619(7)$ & 1.4598 \\
\hline $\mathrm{C}_{2}=\mathrm{C}_{3}$ & $1.3981(2)$ & $1.4009(7)$ & $1.3934(3)$ & 1.3951 \\
\hline $\mathrm{C}_{3}-\mathrm{C}_{4}$ & $1.4003(2)$ & $1.4007(6)$ & $1.3948(5)$ & 1.4015 \\
\hline $\mathrm{C}_{4}=\mathrm{C}_{5}$ & $1.3823(1)$ & $1.3811(9)$ & $1.3720(4)$ & 1.3787 \\
\hline BLA & $0.0436(4)$ & $0.0459(8)$ & $0.0474(4)$ & 0.0438 \\
\hline$\theta^{\ddagger}$ & $4.76(5)$ & $4.48(5)$ & 2.63(4) & 1.8 \\
\hline$\gamma^{\#}$ & $72.20(4)$ & $72.4(1)$ & $73.56(4)$ & 75.4 \\
\hline
\end{tabular}

\subsection{Conclusions}

We have developed a novel class of compact multideterminantal Jastrow-Slater wave functions based on localized orbitals that can be progressively extended to higher quality and accurately treat on an equal footing the ground and the excited states in QMC calculations. Since excited states may in general be characterized by charge transfer, the orbitals to correlate in the determinantal expansion cannot be selected based on their spacial vicinity as we had done in our previous work on ground states. To establish a criterion to account for the most important transitions in the excited state, we borrowed the concept of orbital domains from local coupled-cluster methods and applied it to the local orbitals in the active space. For each doubly-occupied reference configuration, an occupied orbital and the set of orbitals coupled to it by large single-electron transitions are grouped in an orbital domain, the dominant domains are identified, and the subsequent excitations constructed within and from the union of the surviving domains. Bonding-antibonding double excitations are always included being the most important configurations after the reference(s) in the ground state. The resulting excitations are collected into classes that are ordered in importance and can then be systematically included in the determinantal component of the Jastrow-Slater wave function.

To further enhance the description of the states of interest (in particular, the excited states), we have also accounted for the presence of multiple Lewis resonance structures in the molecule. For a given representation of the delocalized orbitals in the active space, multiple set of equivalent local orbitals can be built corresponding to the different Lewis structures. These sets are no longer equivalent if we depart from the complete expansion, as we do when using the concept of domains, and each set may offer a better description of the different states in different parts of the poten- 
tial energy surface. We first verified that our scheme of constructing and classifying the orbital domains and excitation classes is transferable to all sets of local orbitals (resonance structure) and then constructed multiple-resonance Jastrow-Slater wave functions as linear combinations of classes of excitations built from the domains of all the different Lewis structures.

The method was demonstrated at the variational and diffusion Monte Carlo level on the vertical excitation energies of the $\pi \rightarrow \pi^{*}$ state of the retinal protonated Schiff base model chromophores, PSBn $(n=2,3,4)$, which represent a challenging system due to the strong intramolecular charge-transfer character of the transition. To test the flexibility of our wave functions, we performed the calculations at two geometries with very different bond-length patterns and therefore better described in terms of different dominant Lewis resonance structures. We found that, while the use of one resonance structure and a small expansion is sufficient in the smallest PSB2 model, the convergence of the excited-state VMC energy with the number of excitation classes becomes increasingly slower for the larger models. On the other hand, if one uses a multi-resonance wave function expanded over all possible Lewis structures, few excitation classes (i.e. the reference(s), important singles, and bondingantibonding doubles) are needed to accurately describe the ground and excited states of the PSBn, yielding total VMC energies in agreement with the reference values obtained with large determinantal expansions. The same behavior is observed at the DMC level as summarized in Table 3.13. Importantly, the same set of excitation classes can be employed to describe both states successfully at the two different geometries considered, whereas one would in general obtain different determinantal expansions when truncating the wave function expressed on delocalized orbitals as often done in QMC calculations. We also employed this compact multi-resonance wave function to determine the relaxed out-of-plane excited-state geometry of PSB3 and obtained geometrical parameters in agreement with the VMC reference calculation. Finally, we showed the additional benefit of the use of local orbitals in the orbital optimization step, whose cost can be significantly reduced by limiting the mixing of the occupied and virtual orbitals based on spacial consideration without sacrificing the quality of the excitation energy.

In summary, we have developed a successful procedure to construct multi-resonance local Jastrow-Slater wave functions that give a balanced description of the ground and the excited states also in systems where distant orbitals are strongly correlated. The resulting expansion is compact and transferable to different geometries without adjustments. For the largest PSB4 model considered here, the size of the domains is still growing but the expansion is already reduced by a factor of 2-3 as compared to the minimum number of determinants in the delocalized-orbital basis, which is needed to achieve converged total energies for both states. As the system grows further, the spatially remote orbitals will become weakly correlated and the size of the domains saturate (see Tables 3.15 and 3.16 in the Appendix for PSB5 and PSB6, respectively), leading to potential significant gains over a calculation using delocalized orbitals. In conclusion, the compactness and accuracy of the expansion, its transferability to different geometries, and the reduction of the orbital optimization cost are very promising features of our novel multi-resonance local wave functions, 
Table 3.13: PSBn: DMC total and excitation energies computed with single- and multi-resonance local wave functions at the in-plane S0 B3LYP equilibrium geometry.

\begin{tabular}{llccc}
\hline Resonance & Determinant (N) & $\begin{array}{c}\text { S0 } \\
\text { (a.u.) }\end{array}$ & $\begin{array}{c}\text { S1 } \\
\text { (a.u.) }\end{array}$ & $\begin{array}{c}\Delta \mathrm{E} \\
(\mathrm{eV})\end{array}$ \\
\hline PSB2 & & & & \\
1 & X0-X3 (9) & $-30.3347(3)$ & $-30.1169(3)$ & $5.93(1)$ \\
1,2 & X0-X3 (20) & $-30.3357(3)$ & $-30.1206(3)$ & $5.85(1)$ \\
Reference $^{*}$ & $(36)$ & $-30.3360(3)$ & $-30.1208(3)$ & $5.86(1)$ \\
\hline PSB3 & & & & \\
1 & X0-X3 (16) & $-42.9010(5)$ & $-42.7377(5)$ & $4.44(2)$ \\
1,2 & X0-X3 (41) & $-42.9042(4)$ & $-42.7454(5)$ & $4.32(2)$ \\
$1,2,3$ & X0-X3 (66) & $-42.9037(4)$ & $-42.7470(4)$ & $4.26(2)$ \\
Reference $^{\dagger}$ & $(282)$ & $-42.9042(4)$ & $-42.7470(4)$ & $4.28(2)$ \\
\hline PSB4 & & & & \\
$1,2,3,4$ & X0-X3 (159) & $-55.4735(4)$ & $-55.3451(4)$ & $3.49(2)$ \\
Reference $^{\ddagger}$ & $(433)$ & $-55.4739(5)$ & $-55.3466(5)$ & $3.46(2)$ \\
\hline
\end{tabular}

* All CAS $(4,4)$ determinants are included.

$\dagger$ All singles, doubles, and triples with the Res- 1 orbitals.

$\ddagger \sim 99 \%$ of CASSCF weights on delocalized natural orbitals.

offering a robust and affordable route to excited-state calculations of large molecular systems in QMC. 


\subsection{Appendix: Domains of larger retinal models}

In Figure 3.5, we show the Res-1 localized CASSCF active orbitals at the groundstate equilibrium geometry of PSB4. The domains of the Res-1 structure are:
a) $\{1: 8\}$
b) $\{2: 7,8\}$
c) $\{3: 6,7,8\}$
d) $\{4: 5,6,7,8\}$

The excitation classes of the Res-1 structure and their corresponding weights are listed in Table 3.14. The three most important single excitations $(3 \rightarrow 7,4 \rightarrow 7$, and $4 \rightarrow 8$ ) indicate a strong charge-transfer character of the $\pi \rightarrow \pi^{*}$ excited state.
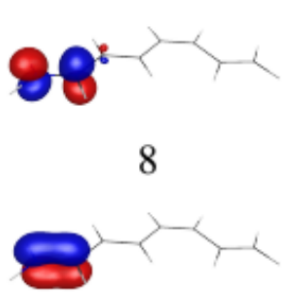

1

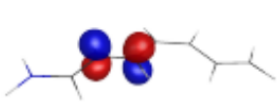

7

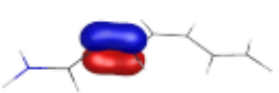

2

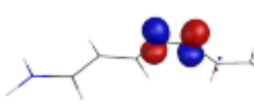

6

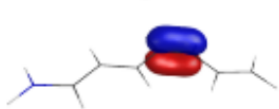

3

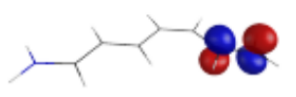

5

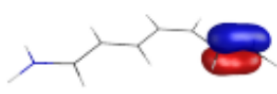

4

Figure 3.5: PSB4: CASSCF localized active orbitals of the Res-1 structure.

Table 3.14: PSB4: CASSCF weight percentage of configurations with the Res-1 orbitals computed at the in-plane S0 B3LYP equilibrium geometry.

\begin{tabular}{|c|c|c|c|c|c|c|c|}
\hline & \multirow[t]{2}{*}{ Type } & \multirow[t]{2}{*}{ Domain } & \multirow[t]{2}{*}{ CSF } & \multirow[t]{2}{*}{ Det } & \multirow{2}{*}{$\begin{array}{l}\text { Configuration } \\
(1,2,3,4,5,6,7,8)\end{array}$} & \multicolumn{2}{|c|}{ Weight (\%) } \\
\hline & & & & & & S0 & S1 \\
\hline X0 & ref & & 1 & 1 & 22220000 & 85.50 & 0.14 \\
\hline \multirow[t]{8}{*}{$\mathrm{X} 1$} & $\mathrm{~S}$ & b & 1 & 2 & 21220001 & 0.25 & 7.53 \\
\hline & S & b & 1 & 2 & 21220010 & 0.00 & 6.57 \\
\hline & S & c & 1 & 2 & 22120001 & 0.28 & 11.35 \\
\hline & S & c & 1 & 2 & 22120010 & 0.38 & 19.31 \\
\hline & S & c & 1 & 2 & 22120100 & 0.00 & 6.20 \\
\hline & $S$ & d & 1 & 2 & 22210001 & 0.11 & 6.54 \\
\hline & $S$ & d & 1 & 2 & 22210010 & 0.13 & 11.92 \\
\hline & $S$ & d & 1 & 2 & 22210100 & 0.11 & 7.17 \\
\hline Total & & & 8 & 16 & & 1.26 & 76.59 \\
\hline \multirow[t]{2}{*}{$\mathrm{X} 2$} & S & $\mathbf{a}$ & 1 & 2 & 12220001 & 0.00 & 1.41 \\
\hline & S & d & 1 & 2 & 22211000 & 0.00 & 1.07 \\
\hline Total & & & 2 & 4 & & 0.00 & 2.48 \\
\hline
\end{tabular}


We also analyze the state-average local wave functions of the larger PSB5 and PSB6 models, whose CASSCF active orbitals are depicted in Figures 3.6 and 3.7, respectively. The ten most important single excitations for PSB5 and PSB6 are listed in Tables 3.15 and 3.16, respectively. The weight analysis of important single excitations indicates that the separated bonding-antibonding singles are still very important. Thus, the intramolecular charge-transfer character of the $\pi \rightarrow \pi^{*}$ state persists and the negative charge is moving from the $\mathrm{C}$-terminus to the $\mathrm{N}$-terminus.

Importantly, we observe that going from PSB4 to PSB5 to PSB6, the single excitation involving the remotest orbitals becomes the sixth, the seventh, and the sixteenth in the order of importance, while the most important excited-state determinants are either adjacent single excitation (in PSB4) or single excitations from a bonding to an antibonding orbitals separated by two atoms (in PSB5 and PSB6). This indicates that, as the system grows, the size of the domain saturates and the resulting excitation classes remain of reasonable size.

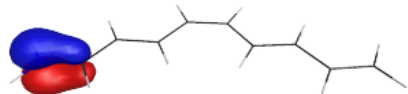

1

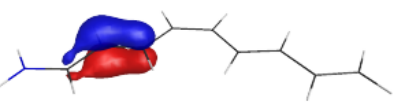

2

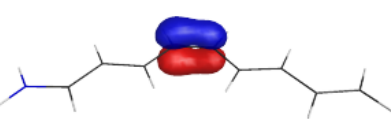

3

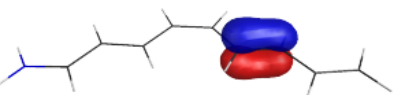

4

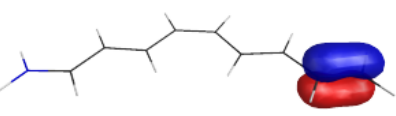

5

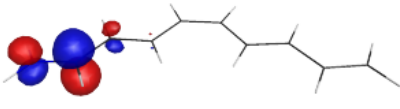

10

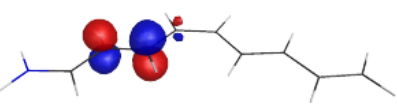

9

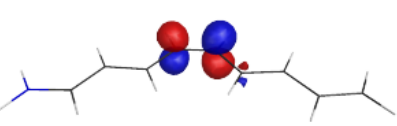

8

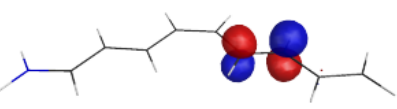

7

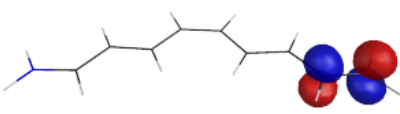

6

Figure 3.6: PSB5: CASSCF localized active orbitals of the Res-1 structure. 


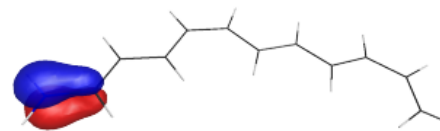

1

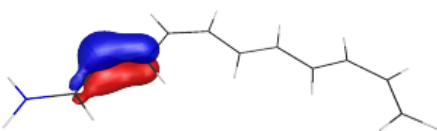

2

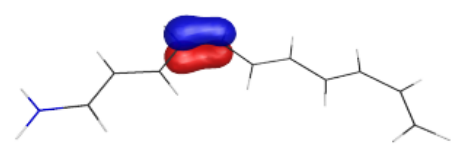

3

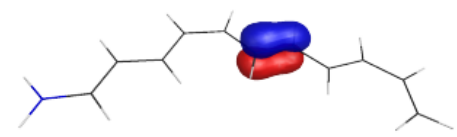

4

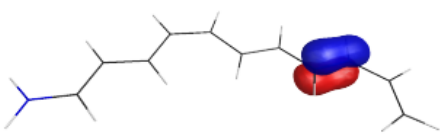

5

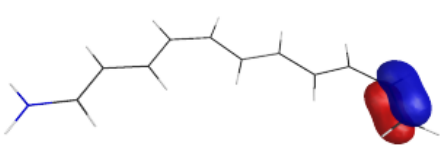

6

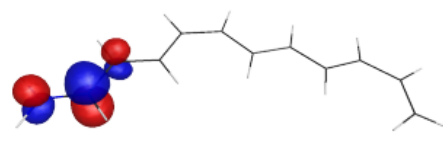

12

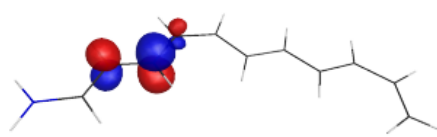

11

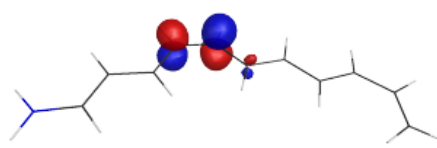

10

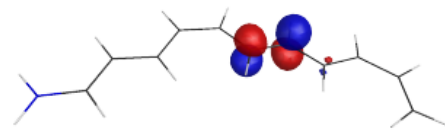

9

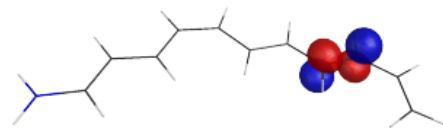

8

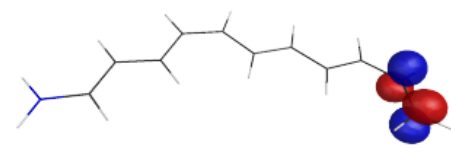

7

Figure 3.7: PSB6: CASSCF localized active orbitals of the Res-1 structure. 
Table 3.15: PSB5: CASSCF weight percentage of the reference and important single configurations with localized Res-1 orbitals computed at the in-plane S0 B3LYP equilibrium geometry.

\begin{tabular}{rrrcrr}
\hline & CSF & Det & Configuration & \multicolumn{2}{c}{ Weight $(\%)$} \\
\cline { 5 - 6 } & & & $(1,2,3,4,5,6,7,8,9,10)$ & S0 & S1 \\
\hline $\mathrm{X0}$ & 1 & 1 & 2222200000 & 81.57 & 0.06 \\
\hline $\mathrm{X} 1$ & 1 & 2 & 2222100001 & 0.08 & 4.96 \\
& 1 & 2 & 2222100010 & 0.03 & 3.14 \\
& 1 & 2 & 2221200001 & 0.26 & 11.60 \\
& 1 & 2 & 2221200010 & 0.12 & 7.47 \\
& 1 & 2 & 2221200100 & 0.09 & 5.60 \\
& 1 & 2 & 2221201000 & 0.01 & 2.61 \\
& 1 & 2 & 2212200001 & 0.29 & 12.90 \\
& 1 & 2 & 2212200010 & 0.14 & 7.77 \\
& 1 & 2 & 2212200100 & 0.03 & 2.81 \\
1 & 2 & 2122200001 & 0.07 & 6.06 \\
\hline
\end{tabular}

Table 3.16: PSB6: CASSCF weight percentage of the reference and important single configurations with localized Res-1 orbitals computed at the in-plane S0 B3LYP equilibrium geometry.

\begin{tabular}{cccccc}
\hline & CSF & Det & Configuration & \multicolumn{2}{c}{ Weight $(\%)$} \\
\cline { 5 - 6 } & & $(1,2,3,4,5,6,7,8,9,10,11,12)$ & S0 & S1 \\
\hline X0 & 1 & 1 & 222222000000 & 77.02 & 0.08 \\
\hline & 1 & 2 & 222212000001 & 0.12 & 3.60 \\
& 1 & 2 & 222212000010 & 0.08 & 5.45 \\
& 2 & 222212000100 & 0.08 & 4.51 \\
& 2 & 222122000001 & 0.14 & 5.39 \\
& 2 & 2 & 222122000010 & 0.14 & 8.20 \\
1 & 2 & 222122000100 & 0.13 & 6.51 \\
1 & 2 & 221222000001 & 0.18 & 5.16 \\
1 & 2 & 221222000010 & 0.18 & 7.22 \\
1 & 2 & 221222000100 & 0.02 & 3.15 \\
1 & 2 & 212222000001 & 0.15 & 2.89 \\
\hline
\end{tabular}




\section{Bibliography}

[1] P. Pulay, Chem. Phys. Lett. 100, 151 (1983).

[2] C. Hampel and H.-J. Werner, J. Chem. Phys. 104, 6286 (1996).

[3] M. Schütz and H.-J. Werner, J. Chem. Phys. 114, 661 (2001).

[4] B. Bories, D. Maynau, and M.-L. Bonnet, J. Comput. Chem. 28, 632 (2007).

[5] F. Neese, F. Wennmohs, and A. Hansen, J. Chem. Phys. 130, 114108 (2009).

[6] F. Neese, A. Hansen, and D. G. Liakos, J. Chem. Phys. 131, 064103 (2009).

[7] S. Saebo and P. Pulay, Annu. Rev. Phys. Chem. 44, 213 (1993).

[8] P. Reinhardt, H. Zhang, J. Ma, and J.-P. Malrieu, J. Chem. Phys. 129, 164106 (2008).

[9] H. Zhang, J.-P. Malrieu, P. Reinhardt, and J. Ma, J. Chem. Phys. 132, 034108 (2010).

[10] C. Riplinger and F. Neese, J. Chem. Phys. 138, 034106 (2013).

[11] C. Riplinger, B. Sandhoefer, A. Hansen, and F. Neese, J. Chem. Phys. 139, 134101 (2013).

[12] P. E. Maslen and M. Head-Gordon, Chemi. Phys. Lett. 283, 102 (1998).

[13] P. E. Maslen and M. Head-Gordon, J. Chem. Phys. 109, 7093 (1998).

[14] G. E. Scuseria and P. Y. Ayala, J. Chem. Phys. 111, 8330 (1999).

[15] M. Schütz, G. Hetzer, and H.-J. Werner, J. Chem. Phys. 111, 5691 (1999).

[16] N. B. Amor, F. Bessac, S. Hoyau, and D. Maynau, J. Chem. Phys. 135, 014101 (2011).

[17] C. Chang, C. J. Calzado, N. B. Amor, J. S. Marin, and D. Maynau, J. Chem. Phys. 137, 104102 (2012).

[18] D. Walter, A. Venkatnathan, and E. A. Carter, J. Chem. Phys. 118, 8127 (2003). 
[19] T. S. Chwee, A. B. Szilva, R. Lindh, and E. A. Carter, J. Chem. Phys. 128, 224106 (2008).

[20] D. B. Krisiloff and E. A. Carter, Phys. Chem. Chem. Phys. 14, 7710 (2012).

[21] O. Demel, J. Pittner, and F. Neese, J. Chem. Theory Comput. 11, 3104 (2015).

[22] T. Korona and H.-J. Werner, J. Chem. Phys. 118, 3006 (2003).

[23] T. D. Crawford and R. A. King, Chem. Phys. Lett. 366, 611 (2002).

[24] D. Kats, T. Korona, and M. Schütz, J. Chem. Phys. 125, 104106 (2006).

[25] B. Helmich and C. Hättig, J. Chem. Phys. 135, 214106 (2011).

[26] B. Helmich and C. Hättig, J. Chem. Phys. 139, 084114 (2013).

[27] T. S. Chwee and E. A. Carter, J. Chem. Theory Comput. 7, 103 (2010).

[28] F. Fracchia, C. Filippi, and C. Amovilli, J. Chem. Theory Comput. 8, 1943 (2012).

[29] F. Fracchia, C. Filippi, and C. Amovilli, J. Chem. Theory Comput. 9, 3453 (2013).

[30] F. Fracchia, C. Filippi, and C. Amovilli, J. Comput. Chem. 35, 30 (2014).

[31] A. G. Anderson and W. A. Goddard III, J. Chem. Phys. 132, 164110 (2010).

[32] B. Braïda, J. Toulouse, M. Caffarel, and C. Umrigar, J. Chem. Phys. 134, 084108 (2011).

[33] CHAMP is a quantum Monte Carlo program package written by Umrigar, C. J.; Filippi, C. and collaborators.

[34] M. Burkatzki, C. Filippi, and M. Dolg, J. Chem. Phys. 126, 234105 (2007).

[35] For the hydrogen atom, we use a more accurate BFD pseudopotential and basis set. M. Dolg and C. Filippi, private communication.

[36] R. A. Kendall, T. H. Dunning Jr, and R. J. Harrison, J. Chem. Phys. 96, 6796 (1992).

[37] C. Filippi and C. J. Umrigar, J. Chem. Phys. 105, 213 (1996), as Jastrow correlation factor, we use the exponential of the sum of three fifth-order polynomials of the electron-nuclear (e-n), the electron-electron (e-e). The Jastrow factor is adapted to deal with pseudo-atoms, and the scaling factor $\kappa$ is set to 0.6 a.u. The 2-body Jastrow factor includes five parameters in the e-e terms and four parameters for each atom type in the e-n terms. 
[38] C. J. Umrigar, J. Toulouse, C. Filippi, S. Sorella, and R. G. Hennig, Phys. Rev. Lett. 98, 110201 (2007).

[39] C. Filippi, M. Zaccheddu, and F. Buda, J. Chem. Theory Comput. 5, 2074 (2009).

[40] M. Casula, Phys. Rev. B 74, 161102 (2006).

[41] J. Pipek and P. G. Mezey, J. Chem. Phys. 90, 4916 (1989).

[42] S. F. Boys, Rev. Mod. Phys. 32, 296 (1960).

[43] J. M. Foster and S. F. Boys, Rev. Mod. Phys. 32, 300 (1960).

[44] C. Edmiston and K. Ruedenberg, Rev. Mod. Phys. 35, 457 (1963).

[45] F. Aquilante, L. De Vico, N. Ferré, G. Ghigo, P.-Å. Malmqvist, P. Neogrády, T. B. Pedersen, M. Pitoňák, M. Reiher, B. O. Roos, L. Serrano-Andrés, M. Urban, V. Veryazov, and R. Lindh, J. Comput. Chem. 31, 224 (2010).

[46] M. W. Schmidt, K. K. Baldridge, J. A. Boatz, S. T. Elbert, M. S. Gordon, J. H. Jensen, S. Koseki, N. Matsunaga, K. A. Nguyen, S. Su, T. L. Windus, M. Dupuis, and J. A. Montgomery, J. Comput. Chem. 14, 1347 (1993).

[47] M. S. Gordon and M. W. Schmidt, in Theory and Applications of Computational Chemistry: the first forty years, edited by C. Dykstra, G. Frenking, K. Kim, and G. Scuseria (Elsevier, 2011), Chap. 41, pp. 1167-1190.

[48] K. Andersson, P.-Å. Malmqvist, B. O. Roos, A. J. Sadlej, and K. Wolinski, J. Phys. Chem. 94, 5483 (1990).

[49] K. Andersson, P.- ^̊. Malmqvist, and B. O. Roos, J. Chem. Phys. 96, 1218 (1992).

[50] J. Finley, P.-Å. Malmqvist, B. O. Roos, and L. Serrano-Andrés, Chem. Phys. Lett. 288, 299 (1998).

[51] F. Aquilante, P.- $\mathrm{A}$. Malmqvist, T. B. Pedersen, A. Ghosh, and B. O. Roos, J. Chem. Theory Comput. 4, 694 (2008).

[52] G. Ghigo, B. O. Roos, and P.-Å. Malmqvist, Chem. Phys. Lett. 396, 142 (2004).

[53] A. D. Becke, Phys. Rev. A. 38, 3098 (1988).

[54] C. Lee, W. Yang, and R. G. Parr, Phys. Rev. B. 37, 785 (1988).

[55] A. D. Becke, J. Chem. Phys. 98, 5648 (1993). 
[56] M. J. Frisch, G. W. Trucks, H. B. Schlegel, G. E. Scuseria, M. A. Robb, J. R. Cheeseman, G. Scalmani, V. Barone, B. Mennucci, G. A. Petersson, H. Nakatsuji, M. Caricato, X. Li, H. P. Hratchian, A. F. Izmaylov, J. Bloino, G. Zheng, J. L. Sonnenberg, M. Hada, M. Ehara, K. Toyota, R. Fukuda, J. Hasegawa, M. Ishida, T. Nakajima, Y. Honda, O. Kitao, H. Nakai, T. Vreven, J. A. Montgomery, Jr., J. E. Peralta, F. Ogliaro, M. Bearpark, J. J. Heyd, E. Brothers, K. N. Kudin, V. N. Staroverov, R. Kobayashi, J. Normand, K. Raghavachari, A. Rendell, J. C. Burant, S. S. Iyengar, J. Tomasi, M. Cossi, N. Rega, J. M. Millam, M. Klene, J. E. Knox, J. B. Cross, V. Bakken, C. Adamo, J. Jaramillo, R. Gomperts, R. E. Stratmann, O. Yazyev, A. J. Austin, R. Cammi, C. Pomelli, J. W. Ochterski, R. L. Martin, K. Morokuma, V. G. Zakrzewski, G. A. Voth, P. Salvador, J. J. Dannenberg, S. Dapprich, A. D. Daniels, Ö. Farkas, J. B. Foresman, J. V. Ortiz, J. Cioslowski, and D. J. Fox, Gaussian 09 Revision D.01, gaussian Inc. Wallingford CT 2009. 


\section{Chapter 4}

\section{Photo-induced mechanism of isomerization of Donor-Acceptor Stenhouse Adducts ${ }^{\dagger}$}

We propose here a complete thermal path of isomerization of first- and secondgeneration donor-acceptor Stenhouse adduct (DASA) photoswitches that can qualitatively explain available experimental observations. Using density functional theory and an implicit solvent model for polar (methanol) and nonpolar (toluene) solvents, we investigate two DASA molecules, namely, an amine-based DASA (named here DASA-1) where the photoisomerization yields a zwitterionic final product and an aniline-based DASA (named DASA-2) resulting in a neutral diketo end product. In both solvents, we find that the all-trans open isomer of both DASAs successfully cyclizes to the closed form with a faster reaction occurring in toluene. In DASA-1, the photoswitching is irreversible in methanol since the zwitterionic end product is lower in energy than the initial isomer, while it is reversible in toluene yielding the zwitterionic product at lower temperature and a possible neutral diketo product that can be formed at higher temperature. In DASA-2, the zwitterionic/diketo product is destabilized/stabilized in both solvents as compared to DASA-1, resulting in (i) an increased importance of the tautomerization pathway toward the diketo final product, (ii) the reversibility of photoswitching in methanol where the two tautomerization pathways can be controlled by changing the temperature, and (iii) a strong preference to reach the diketo product in toluene.

Furthermore, our preliminary study of the actinic step in DASA using multiconfigurational wave-function methods reveals that the inclusion of dynamical electron correlation in constructing the excited-state potential energy surface is mandatory. The sole account for static electron correlation yields a counterintuitive barrierless relaxation of the excited state of the reactant to its own ground state, due to a rotation around the formal double bond closest to the acceptor moiety. Interestingly, based on

\footnotetext{
${ }^{\dagger}$ This chapter is in preparation as H. Zulfikri and C. Filippi, "Investigating the thermal step of the photoswitching mechanism of donor-acceptor Stenhouse adducts: Electrocyclization and subsequent forking of tautomerization pathways", and "On the actinic step of donor-acceptor Stenhouse adduct photoswitch: A CASSCF and CASPT2 study".
} 
the existence of a minimum in the excited-state potential energy surface computed at a higher level of (perturbation based) theory, we infer the presence of a barrier for rotation around any formal double bonds. Further calculations with higher correlated methods are needed.

\subsection{Introduction}

Donor-acceptor Stenhouse adducts (DASAs) are a novel class of synthetic photoswitches which has acquired significant interest since its first discovery in 2014 [1,2]. They consist of either an amine-based (first generation) or an aniline-based (second generation) [3,4] electron-rich donor and a 1,3-dicarbonyl electron-deficient acceptor moiety connected with a conjugated triene bridge. Upon visible-light irradiation, they undergo a ring-closure reaction from a linear colored structure to a compact colorless isomer. This negative photochromic feature coupled with simple synthetic procedures have lead to many applications of DASAs in material science [5-10] and to their pioneering use in biological systems [11].

In order to fully realize DASAs' potential, a detailed molecular understanding of the complete photoisomerization reaction mechanism is required. A recent UV/Vis spectroscopy experiment has established that the overall isomerization reaction can be divided into an actinic and a thermal step [12]. Further structural elucidation using ultrafast visible and mid-IR spectroscopy in combination with density functional theory (DFT) calculations has concluded that, upon irradiation with visible light, the initial $\mathbf{A}$ form isomerizes to the $\mathbf{A}^{\prime}$ configuration through a rotation around the middle double bond of the triene in solvents such as dichloromethane and chloroform [13], as illustrated in Figure 4.1. The same behavior is also observed in toluene and methanol solutions [14]. Since further irradiation of $\mathbf{A}^{\prime}$ results in the backward cis-trans isomerization, it is inferred that the reaction from $\mathbf{A}^{\prime}$ to the closed $\mathbf{B}$ form proceeds thermally. The $\mathbf{B}$ isomer is however not the final product of the forward switching reaction: tautomerization takes place to generate two different outcomes, namely, the zwitterionic $\mathbf{B}^{\prime \prime \prime}$ and the neutral $\mathbf{C}$ for the amine- and the aniline-based DASAs, respectively.

Using DFT and an implicit solvent model in both nonpolar (toluene) and polar (methanol) solvents, we unravel the complete thermal step of a DASA photoswitch with a dimethylamine donor and a Meldrum acid acceptor (named here DASA-1), starting from the product of the actinic step, $\mathbf{A}^{\prime}$, and ending with the zwitterionic closed form, $\mathbf{B}^{\prime \prime \prime}$. For the tautomerization step, we investigate the possible presence of an intramolecular proton-transfer pathway, which we expect to be faster than a mechanism with direct solvent involvement, requiring instead a network of solvent molecules in specific arrangements from the proton donor to the acceptor. The competing enol-keto tautomerization pathway that leads to $\mathbf{C}$ is also and studied. Furthermore, we perform similar calculations for a second-generation DASA photoswitch with a 5-methoxyindoline donor and a Meldrum acid acceptor (named DASA-2) in which the full forward switching yields primarily the $\mathbf{C}$ isomer. The Lewis structure of the two DASA molecules studied in this work is shown in Figure 4.2. 


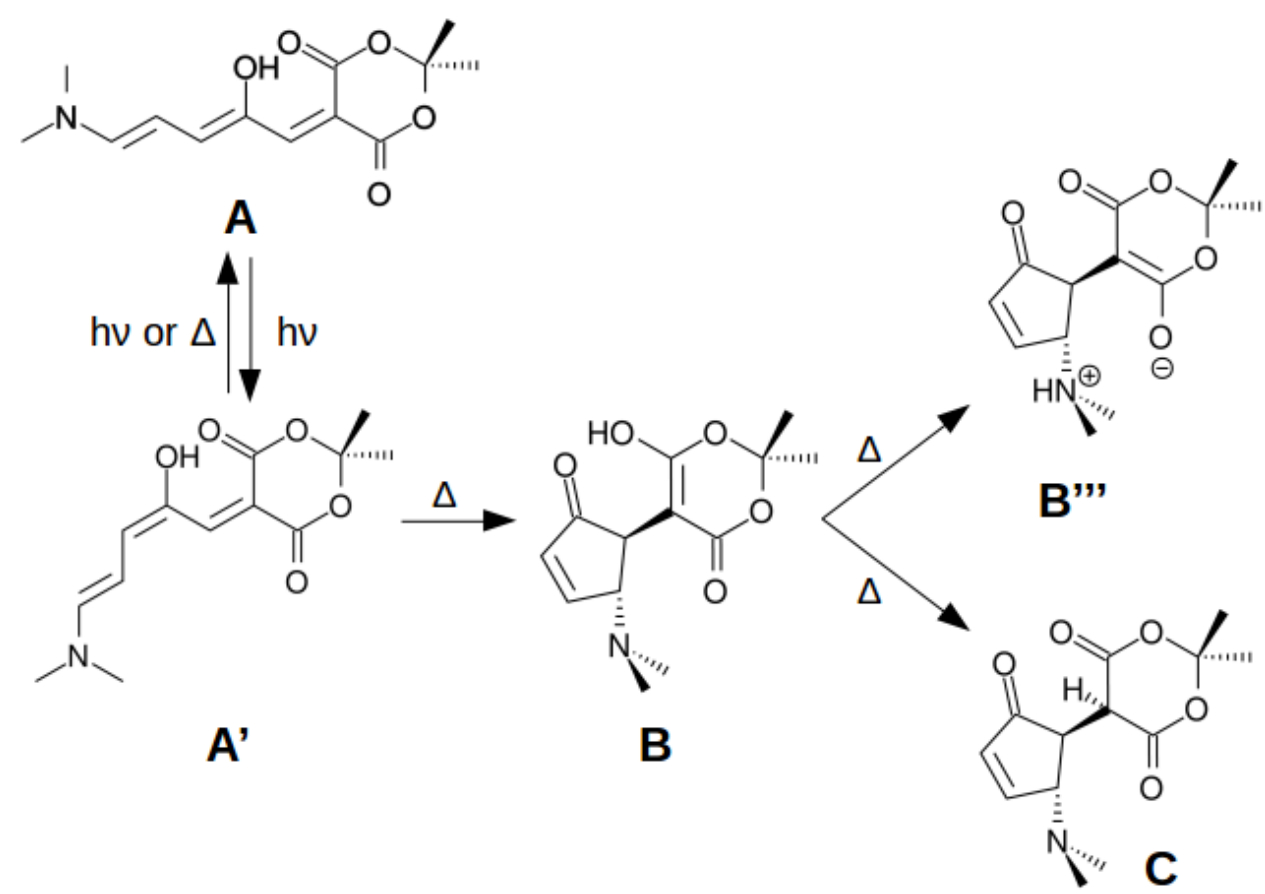

Figure 4.1: Photoisomerization of DASA in solution phase depicted for dimethylamine Meldrum DASA. The back switching is only shown for the actinic step.<smiles>CN(C)/C=C/C=C(\O)C=C1C(=O)OC(C)(C)OC1=O</smiles>

DASA-1<smiles>COc1ccc2c(c1)CCN2/C=C/C=C(\O)C=C1C(=O)OC(C)(C)OC1=O</smiles>

DASA-2

Figure 4.2: Two DASA molecules studied in this work. They differ in the type of donor: DASA-1 has an amine group while DASA-2 has an aniline-based donor extending the $\pi$ conjugation.

Finally, we present a preliminary study of the actinic step between $\mathbf{A}$ and $\mathbf{A}^{\prime}$ using multiconfigurational wave-function methods in vacuum, which is of relevance to explain experimental observations in nonpolar solvents such as toluene. Our exploration of the excited-state potential energy surface represents an important first step to establish the needed computational ingredients for the study of DASA photochemistry, especially since theoretical work in the literature has so far only focused on the absorption properties of open isomers [15-17].

\subsection{Computational details}

To explore the thermal mechanism, we employ the M06-2X hybrid functional [18] together with a reduced version of the augmented cc-pVDZ basis set in which only 
the $s$ and $p$ diffuse functions are added for the non-hydrogen atoms [19]. This basis set (denoted as maug-cc-pVDZ) has been shown to be adequate for DFT calculations of the type of barrier heights and noncovalent interactions [20] also present in our proposed reaction pathways. The solvation model based on density (SMD) scheme [21] is used to mimic the solvent. Geometries are optimized with a tight convergence criteria, i.e. until the root mean square of the force is less than $10^{-5}$ a.u. We perform frequency calculations to determine the nature of the resulting stationary points: reactants and products are characterized by positive frequencies, while transition states have a single imaginary vibrational frequency with direction corresponding to the movement along the reaction coordinate under investigation. To further validate our conclusions, we repeat all calculations with the $\omega$ B97X-D rangeseparated hybrid functional [22]. Both the M06-2X and the $\omega$ B97X-D functional have been extensively investigated and compared against 200 other functionals for the study of a wide range of ground-state reactions of organic compounds, and have been found to give reliable results for this class of problems [23]. Additionally, we also employ the popular B3LYP functional [24-26]. All ground-state DFT computations are performed with the Gaussian09 software [27].

The excited-state relaxation pathways are studied using a state-averaged completeactive-space self-consistent-field (SA-CASSCF) method [28] with equal weights over the states of interest and the multi-state complete-active-space second-order perturbation theory (MS-CASPT2) method [29-31] to account for dynamical electron correlation. From the Franck-Condon geometry, the minimum energy path (MEP) is calculated with a radius of hypersphere of either 0.05 or 0.075 a.u. The gradients of the electronic energy with respect to nuclear coordinates are computed analytically for the CASSCF wave functions [32] and numerically for the CASPT2 method. In the CASPT2 calculations, we employ the default zero-order Hamiltonian [33] with an IPEA shift of 0.25 a.u. and, to eliminate intruder-state problems, an imaginary level shift [34] of 0.2 a.u. is used. The Cholesky decomposition of the two-electron integrals [35] with a default threshold of $10^{-4}$ is used to speed up the CASPT2 calculations. All excited-state calculations use the $6-31 \mathrm{G}^{*}$ basis set with Cartesian $d$ polarization functions and are performed with the Molcas 8.0 package [36].

\subsection{Results and discussions}

In this Section, we first investigate the thermal relaxation path of DASA-1 with the M06-2X/maug-cc-pVDZ procedure and compare our findings with experimental observations. We then consider DASA-2 and discuss the results against those of DASA1. Finally, a preliminary excited-state investigation of the actinic step is reported at the end of this Section.

\subsubsection{Thermal step of DASA-1}

As detailed in Figure 4.3, after relaxation from the excited state of $\mathbf{A}$ to the ground state of $\mathbf{A}^{\prime}$, a thermally-allowed single-bond rotation around the $\mathrm{C}_{2}-\mathrm{C}_{3}$ bond is re- 


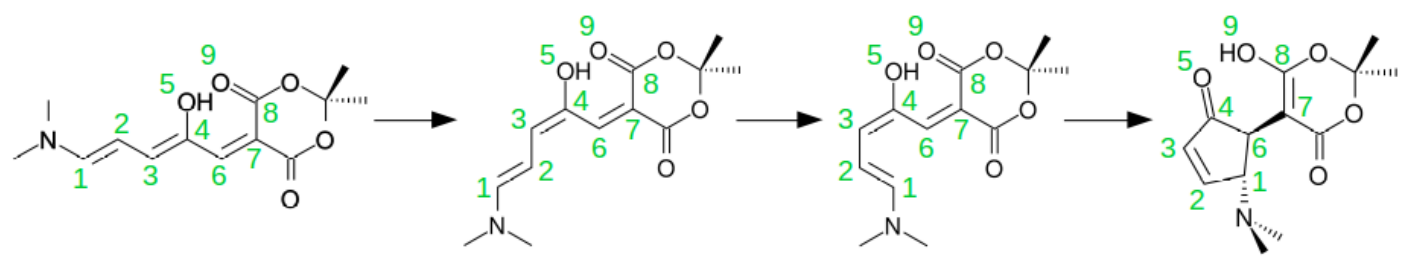

A

$A^{\prime}$

A"

B

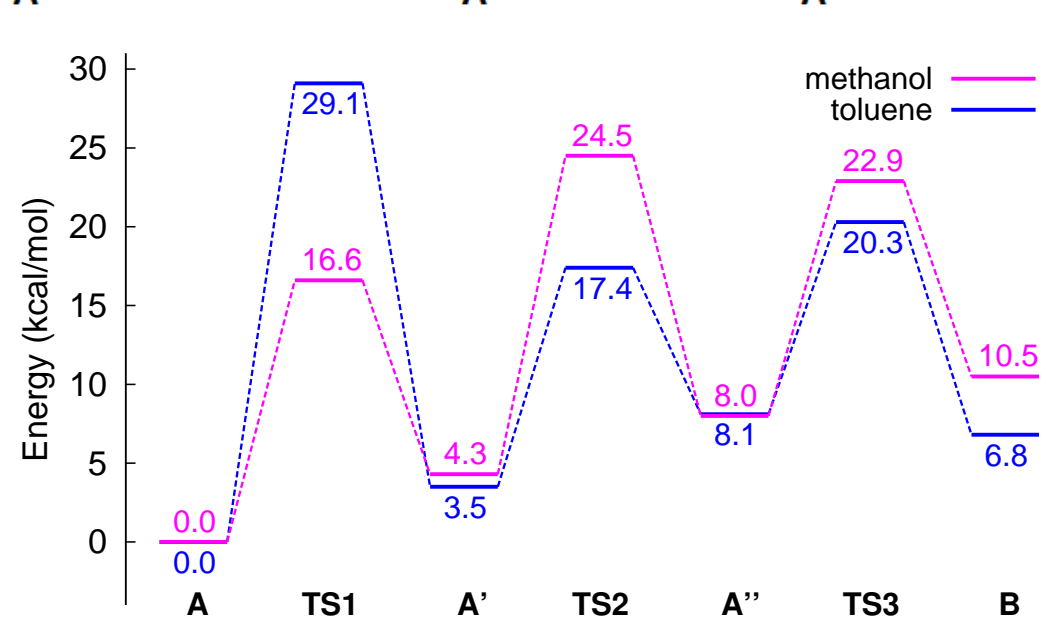

Figure 4.3: Ring-closure reaction (top) and Gibbs free energies (bottom) of all minima and transition states in methanol (purple) and toluene (blue) solvents. Atoms are numbered in green.
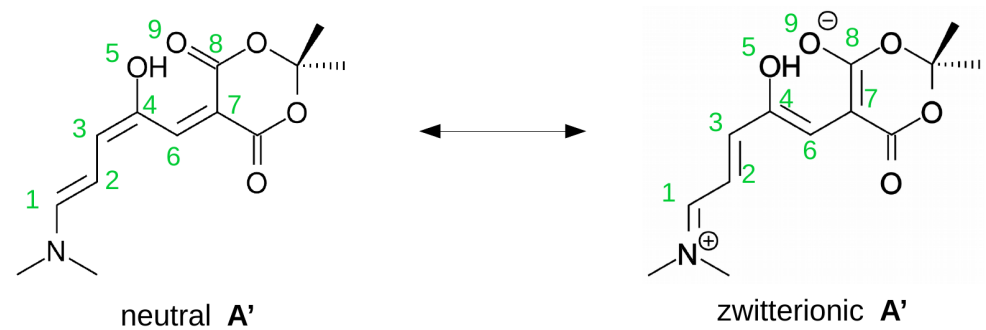

Figure 4.4: The two dominant resonance structures of the $\mathbf{A}^{\prime}$ isomer.

quired to bring the $\mathrm{C}_{1}$ and $\mathrm{C}_{6}$ atoms in close proximity and, thus, enable the ringclosure reaction. The triene system of the resulting $\mathbf{A}^{\prime \prime}$ form is not as planar as the $\mathbf{A}$ and $\mathbf{A}^{\prime}$ isomers due to the steric hindrance of the two hydrogen atoms bound to $\mathrm{C}_{1}$ and $\mathbf{C}_{6}$. Interestingly, the relative energies of $\mathbf{A}, \mathbf{A}^{\prime}$ and $\mathbf{A}^{\prime \prime}$ are practically the same in toluene and methanol. Therefore, these open isomers can be evenly stabilized by adjusting the relative contribution of their neutral and zwitterionic resonance structures (shown in Figure 4.4 for $\mathbf{A}^{\prime}$ ) to the solvent polarity. In contrast, the barrier heights of the forward reaction $\mathbf{A}^{\prime} \rightarrow \mathbf{A}^{\prime \prime}$ (via the TS2 transition state) and of the backward reaction $\mathbf{A}^{\prime} \rightarrow \mathbf{A}$ (via TS1) are strongly solvent dependent.

The bond lengths of the A, $\mathbf{A}^{\prime}$ and $\mathbf{A}^{\prime \prime}$ minima and the TS1 and TS2 transition states are shown in Figure 4.5, where we also display the bond-length alternation (BLA) defined as the difference between the averages of the formal single and double 

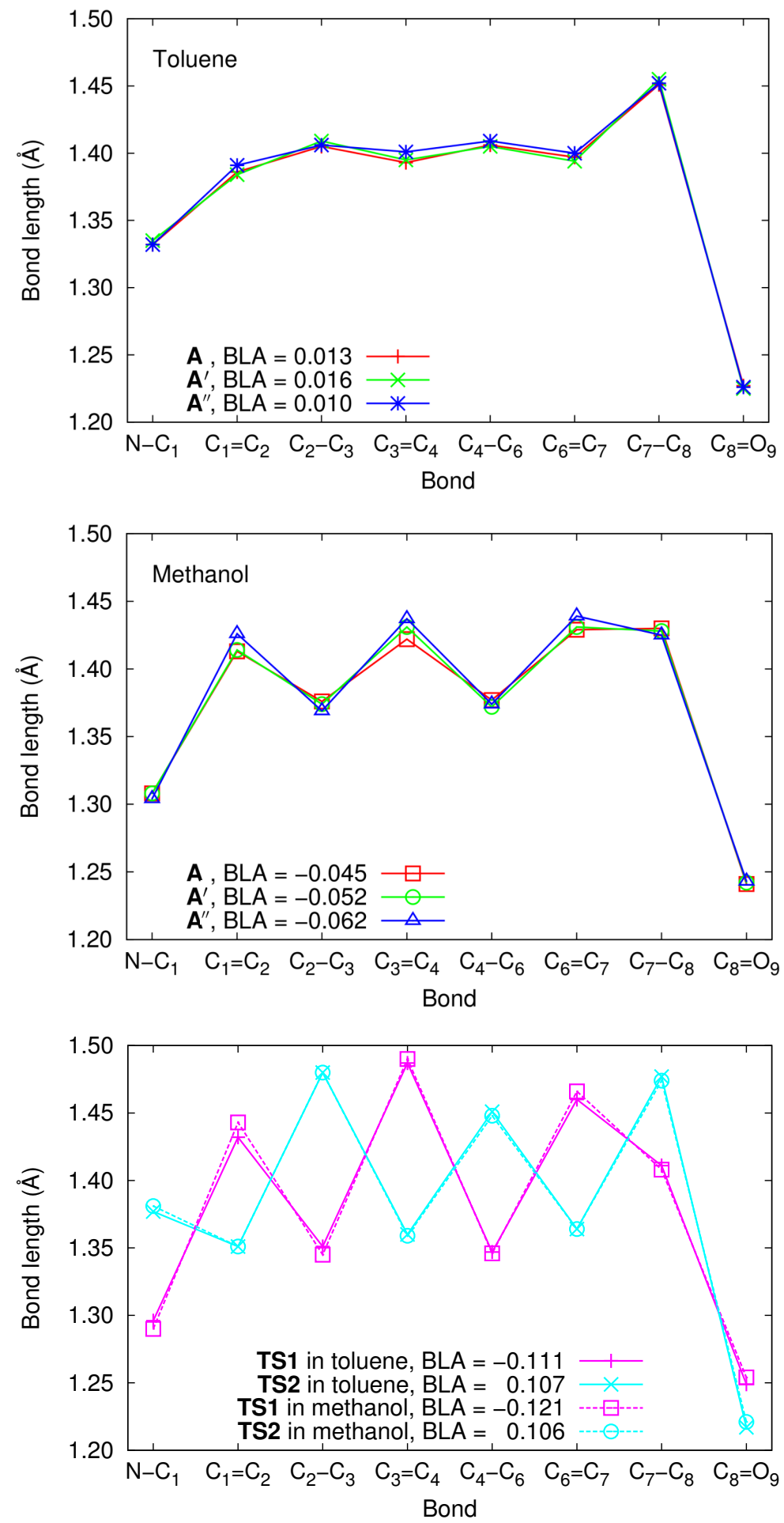

Figure 4.5: Optimal bond lengths ( $⿱$ ) of the minima in toluene (top) and methanol (middle) and of the transition states in both solvents (bottom). Environment only affects the bond length pattern of the minima leaving the bond structure of the transition states unaltered. 
bonds of the triene system as:

$$
\mathrm{BLA}=\frac{1}{2}\left[r\left(\mathbf{C}_{2}-\mathbf{C}_{3}\right)+r\left(\mathbf{C}_{4}-\mathbf{C}_{6}\right)\right]-\frac{1}{3}\left[r\left(\mathbf{C}_{1}=\mathbf{C}_{2}\right)+r\left(\mathbf{C}_{3}=\mathbf{C}_{4}\right)+r\left(\mathbf{C}_{6}=\mathbf{C}_{7}\right)\right] .
$$

We find that the three minima have a similarly small, positive BLA in toluene, while they display a negative BLA in methanol due to the dominance of the zwitterionic structure in this polar solvent. The bonding pattern of the transition states is instead not affected by the solvent polarity. These two findings can explain the solvent-dependent behavior of the barriers between the open isomers. For instance, in toluene, the forward isomerization, $\mathbf{A}^{\prime} \rightarrow \mathbf{A}^{\prime \prime}$, has a lower barrier by $11.7 \mathrm{kcal} / \mathrm{mol}$ than the backward reaction, $\mathbf{A}^{\prime} \rightarrow \mathbf{A}$. In the forward reaction, the BLA evolves from $0.016\left(\mathbf{A}^{\prime}\right)$ to $0.107 \AA$ (TS2), while it undergoes a somewhat larger reduction to $-0.111 \AA$ (TS1) in the backward reaction, reaching a less favorable zwitterionic transition state in such a nonpolar solvent. In methanol, however, the forward reaction involves a significant increase of BLA from $-0.052\left(\mathbf{A}^{\prime}\right)$ to $0.106 \AA$ (TS2), while the decrease to $-0.121 \AA$ (TS1) is smaller in the other direction. Therefore, the thermal barrier for the forward isomerization leading to the ring closure is higher than that for the backward reaction in methanol. This finding correlates with the longer irradiation time needed experimentally for the full conversion to the closed isomers in methanol [1]: one needs to continuously populate the $\mathbf{A}^{\prime}$ isomer to counter the energetically-favored thermal backward reaction, $\mathbf{A}^{\prime} \rightarrow \mathbf{A}$.

In the next step, electrocyclization from $\mathbf{A}^{\prime \prime}$ to a colorless $\mathbf{B}$ isomer occurs concomitantly with a proton-transfer reaction. The mechanism by which the conjugated triene system cyclizes had been anticipated [1] to resemble the Nazarov [37] or the Piancatelli $[38,39]$ reaction, which both require a Lewis acid (e.g. a proton) to activate the ring-closure reaction and the subsequent keto-enol tautomerization. Contrary to this expectation, we find that the $4 \pi$ electrocyclization of $\mathbf{A}^{\prime \prime}$ does not necessitate an external Lewis acid. Moreover, as in a Nazarov-like reaction on a deprotonated Stenhouse salt [40], the cyclization occurs in one step, being in our case accompanied by a concurrent proton transfer. Both the barrier height and the reaction energy from $\mathbf{A}^{\prime \prime}$ to $\mathbf{B}$ are found to be solvent dependent: as the ring cyclizes, the dipole moment decreases, and the compound becomes more stable in nonpolar solvent. The transition state for the ring-closure reaction (TS3) is characterized by a significant reduction of the $\mathrm{C}_{1}-\mathrm{C}_{6}$ distance from about $3.0 \AA$ in $\mathbf{A}^{\prime \prime}$ to $2.0 \AA$, and by the shortest average distance of the bridging hydrogen to both the $\mathrm{O}_{5}$ and the $\mathrm{O}_{9}$ atom. This hydrogen atom is somewhat closer to the $\mathrm{O}_{5}$ atom in this transition state and will immediately be transferred to $\mathrm{O}_{9}$ when the $\mathrm{C}_{1}-\mathrm{C}_{6}$ distance becomes shorter than the value at TS3.

We have now a complete energy profile for the $\mathbf{A}^{\prime} \rightarrow \mathbf{B}$ thermal ring closure of DASA-1 and can identify the rate-limiting step, which is very important in order to further optimize the reaction. Our DFT calculations reveal that the determining step is the $\mathbf{A}^{\prime \prime} \rightarrow \mathbf{B}$ reaction in toluene and the $\mathbf{A}^{\prime} \rightarrow \mathbf{A}^{\prime \prime}$ in methanol. The overall barrier in methanol is also higher by $3.4 \mathrm{kcal} / \mathrm{mol}$ than in toluene, contributing to the slower rate of the full conversion to the close forms in this polar solvent [1]. 

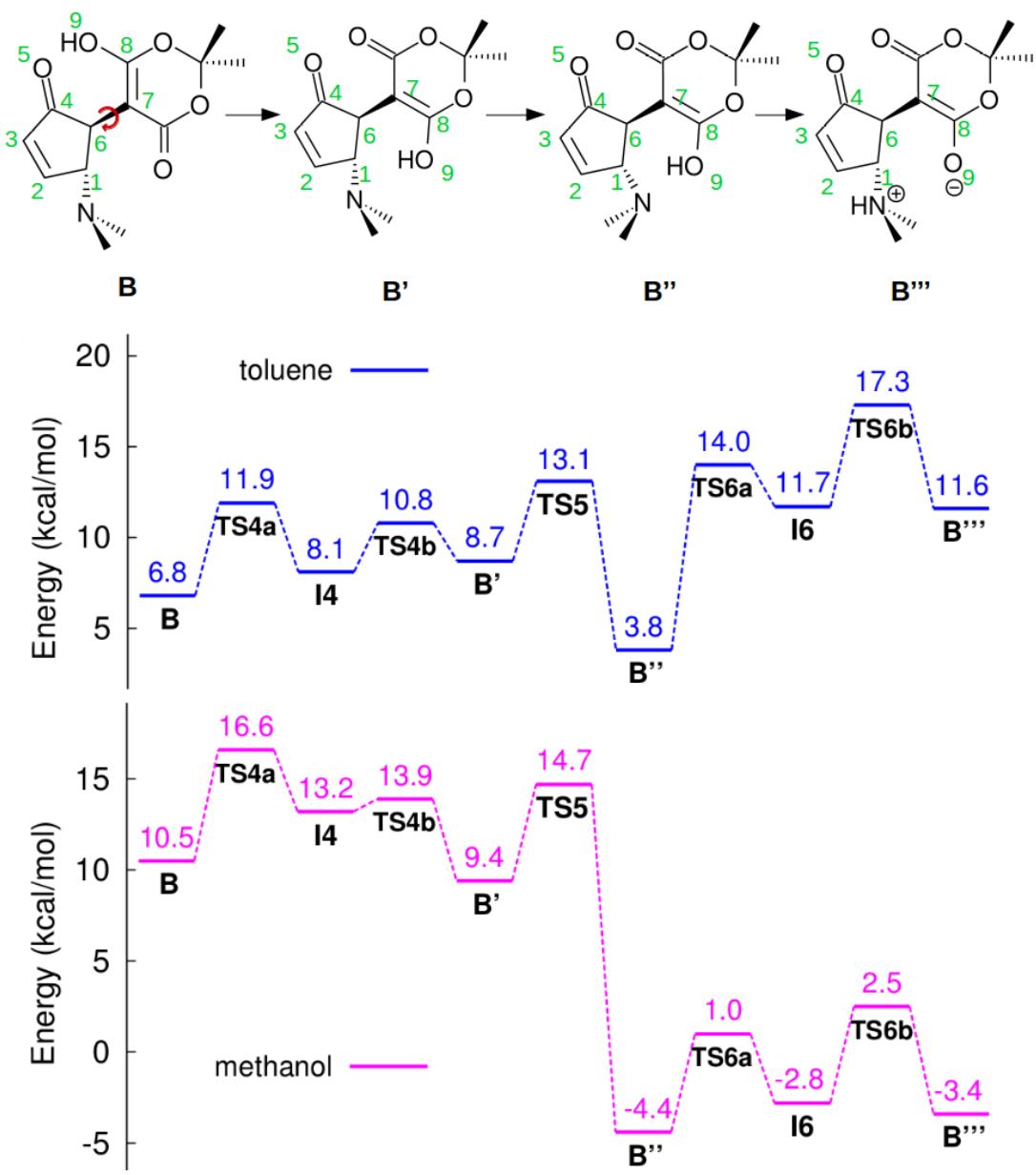

Figure 4.6: Tautomerization reaction from the neutral $\mathbf{B}$ to the zwitterionic $\mathbf{B}^{\prime \prime \prime}$ closed form (top) in toluene (middle) and methanol (bottom).

Since $\mathbf{B}$ is not the final product of the forward switching, we construct three consecutive steps which lead to the zwitterionic $\mathbf{B}^{\prime \prime \prime}$ with the hydrogen atom transferred from the Meldrum moiety to the amine group. The results are shown in Figure 4.6. First, a rotation around the single $\mathrm{C}_{6}-\mathrm{C}_{7}$ bond connecting the cyclopentenone and Meldrum ring transforms $\mathbf{B}$ to $\mathbf{B}^{\prime}$, bringing the alcohol group close to the amine. When rotating the Meldrum ring clockwise with respect to the cyclopentenone, we locate an intermediate $\mathbf{I} 4$ before reaching $\mathbf{B}^{\prime}$. The intramolecular $\mathrm{O}_{5}-\mathrm{O}_{9}$ hydrogen bond is still maintained in $\mathbf{I 4}$ and will eventually be lost in $\mathbf{B}^{\prime}$. We find that the barrier (TS4a) encountered to reach $\mathbf{I} 4$ mainly arises from the steric hindrance associated with the dimethylamine group. The subsequent barrier (TS4b) is smaller and mainly originates from the elongation of the $\mathrm{O}_{5}-\mathrm{O}_{9}$ intramolecular hydrogen-bond distance. We note that the counterclockwise rotation of the Meldrum ring is not favored in toluene as it yields a one-step transformation to $\mathbf{B}^{\prime}$ with a barrier of $10.8 \mathrm{kcal} / \mathrm{mol}$, which is $5.7 \mathrm{kcal} / \mathrm{mol}$ higher than the barrier encountered in the clockwise rotation. On the other hand, in methanol, the direction for this rotation does not matter as the barriers are practically the same (within $0.1 \mathrm{kcal} / \mathrm{mol}$ ). 
In the second step, a nitrogen inversion of the tertiary amine group takes place to transform $\mathbf{B}^{\prime}$ to $\mathbf{B}^{\prime \prime}$. This forward inversion is exothermic in both solvents as it shortens the distance between the $\mathrm{N}$ and $\mathrm{O}_{9}$ atoms enabling the formation of an additional intramolecular hydrogen bond which stabilizes the product. In methanol, this transformation is accompanied by a proton transfer from the $\mathrm{O}_{9}$ to the $\mathrm{N}$ atom, resulting in a zwitterionic $\mathbf{B}^{\prime \prime}$ structure. Consequently, the forward nitrogen inversion in this polar solvent is even more exothermic because of the formation of a product with an extra intramolecular electrostatic attraction between the cationic amine and the enolate group. The resulting $\mathbf{B}^{\prime \prime}$ is energetically lower by $4.4 \mathrm{kcal} / \mathrm{mol}$ than the starting A form, a finding which explains the irreversibility of the overall photoisomerization in methanol [1]. For the forward inversion reaction, we find that the barrier is not influenced by the environment $(5.3$ and $5.4 \mathrm{kcal} / \mathrm{mol}$ for toluene and methanol, respectively), while it is certainly solvent dependent for the backward inversion due to the different stabilization of $\mathbf{B}^{\prime \prime}$ in the two solvents. The corresponding transition state (TS5) is characterized by the full $\mathrm{sp}^{2}$ hybridization of the $\mathrm{N}$ atom as indicated by the sum of the three $\mathrm{C}-\mathrm{N}-\mathrm{C}$ angles being equal to $360^{\circ}$. A weak hydrogen bond with a distance of $3.5 \AA$ between the acceptor $\mathrm{N}$ and the donor $\mathrm{O}_{9}$ is also present at TS5, and in methanol solution (but not in toluene), the proton is transferred to $\mathrm{N}$ beyond the transition state.

We note that the conversion from $\mathbf{B}$ to $\mathbf{B}^{\prime \prime}$ can also proceed by first inverting the $\mathrm{sp}^{3}$ nitrogen atom and then proceeding with a single-bond rotation around the $\mathrm{C}_{6}-\mathrm{C}_{7}$ bond connecting the two rings. We find that this alternative route results in an overall barrier height compatible with the previously discussed reaction path. Therefore, both routes contribute equally in transforming $\mathbf{B}$ to $\mathbf{B}^{\prime \prime}$.

In the last step, a rotation around the $\mathrm{C}_{1}-\mathrm{N}$ single bond converts $\mathbf{B}^{\prime \prime}$ to the final product $\mathbf{B}^{\prime \prime \prime}$, which has been observed in X-ray diffraction experiments on a similar diethylamine Meldrum DASA crystal [1]. In toluene, the rotation is preceded by a proton transfer from the $\mathrm{O}_{9}$ to the $\mathrm{N}$ atom, yielding the zwitterionic product. The $\mathbf{B}^{\prime \prime} \rightarrow \mathbf{B}^{\prime \prime \prime}$ step is not thermodynamically favorable in toluene because it requires to overcome a barrier of $13.5 \mathrm{kcal} / \mathrm{mol}$ towards a final product which is higher in energy by $7.8 \mathrm{kcal} / \mathrm{mol}$. On the other hand, in methanol, the $\mathrm{C}_{1}-\mathrm{N}$ single-bond rotation can easily occur due to the small barrier heights between the initial $\left(\mathbf{B}^{\prime \prime}\right)$, intermediate (I6) and final $\left(\mathbf{B}^{\prime \prime \prime}\right)$ zwitterionic minima, which are also very close in energy.

We also investigate the second thermal route of $\mathbf{B}$ that involves the enol-keto tautomerization and leads to the $\mathbf{C}$ isomer, which is the primary final product of photoswitching of the aniline-based second-generation DASAs. In a Meldrum acid (our DASA-1 molecule without the cyclopentenone ring), this type of tautomerization is characterized by a very high barrier of $52 \mathrm{kcal} / \mathrm{mol}$ in the gas phase, and the reaction would need to be catalyzed by an external acid or base at ambient conditions. Interestingly, we find instead that the tautomerization reaction of DASA-1 can occur intramolecularly without direct involvement of the solvent. As shown in Figure 4.7, the hydrogen is first transferred from $\mathrm{O}_{9}$ to $\mathrm{O}_{5}$ to form an $\mathbf{I 7}$ intermediate without dissociating the $\mathrm{C}_{1}-\mathrm{C}_{6}$ bond. Then, the proton transfer proceeds from the $\mathrm{O}_{5}$ to the $\mathrm{C}_{7}$ atom resulting in a diketo $\mathbf{C}$ product. The $\mathbf{I 7}$ intermediate is stabilized in polar solvents as it bears a zwitterionic character with the Meldrum and cyclopentenone 

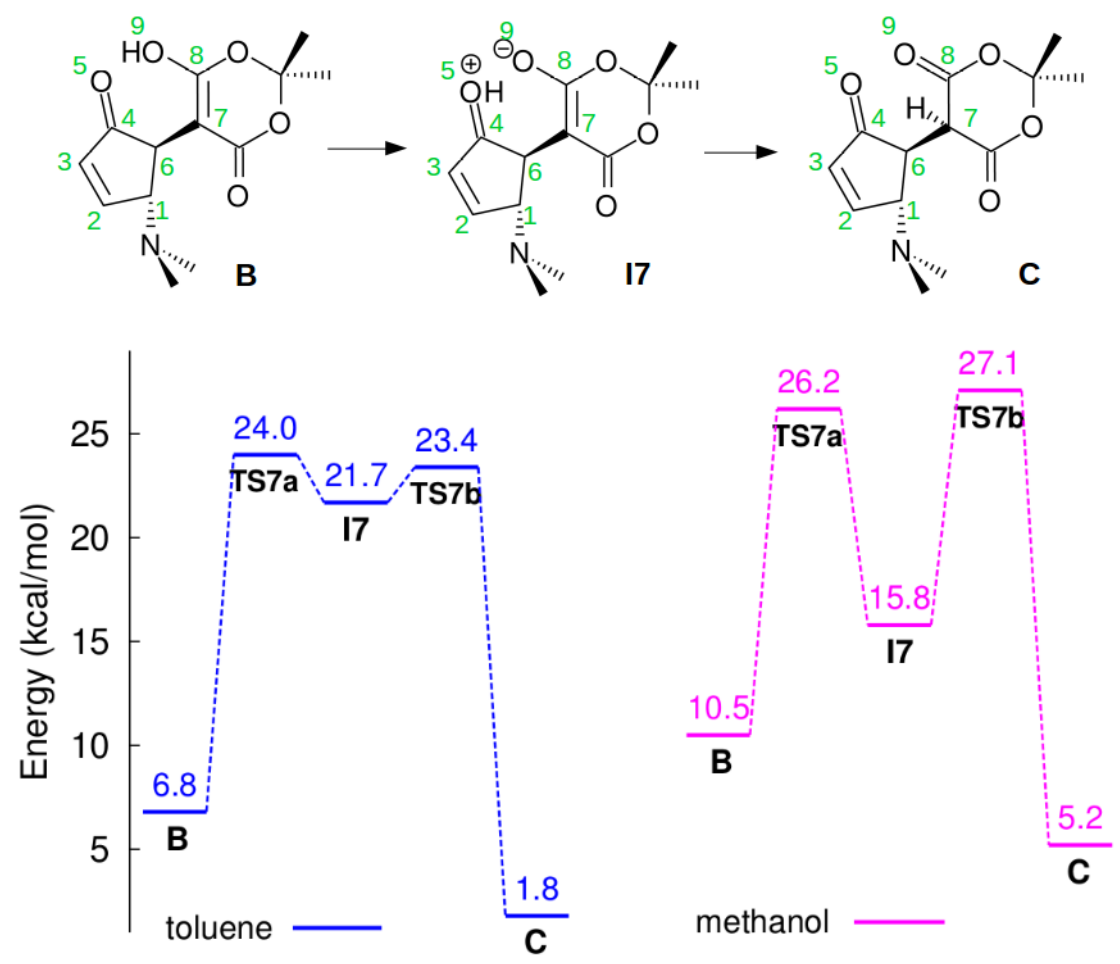

Figure 4.7: Enol-keto tautomerization to the $\mathbf{C}$ neutral closed form in toluene (left) and methanol (right).

rings being negatively and positively charged, respectively. In methanol, both the barrier height and the reaction energy of the pathway to $\mathbf{C}$ are higher than those of the first route yielding $\mathbf{B}^{\prime \prime \prime}$ (by 10.5 and $8.4 \mathrm{kcal} / \mathrm{mol}$, respectively). Our results therefore explain the fact that the $\mathbf{C}$ isomer is not observed among the final products of the forward switching reaction in methanol. In toluene, however, the two tautomerization paths are differently governed by their kinetics and thermodynamics. The first route is preferred at low temperature (kinetically controlled reaction) since the barrier heights to obtain the $\mathbf{B}^{\prime \prime}$ and $\mathbf{B}^{\prime \prime \prime}$ isomers are lower by $6.7 \mathrm{kcal} / \mathrm{mol}$ and $10.9 \mathrm{kcal} / \mathrm{mol}$, respectively, than the overall barrier of the route to $\mathbf{C}$. If the reaction is thermodynamically controlled by increasing the temperature, the $\mathbf{C}$ isomer will be formed considerably since it is lower in energy by at least $2.0 \mathrm{kcal} / \mathrm{mol}$ than all intermediates and products encountered in the first route.

The thermal path of DASA-1 we have computed with the M06-2X/maug-ccpVDZ procedure explains very well several experimental observations. We therefore proceed with the same recipe to unravel the thermal step of DASA-2. For a comparison with the results obtained with other DFT functionals, we refer the reader to the Appendix. 

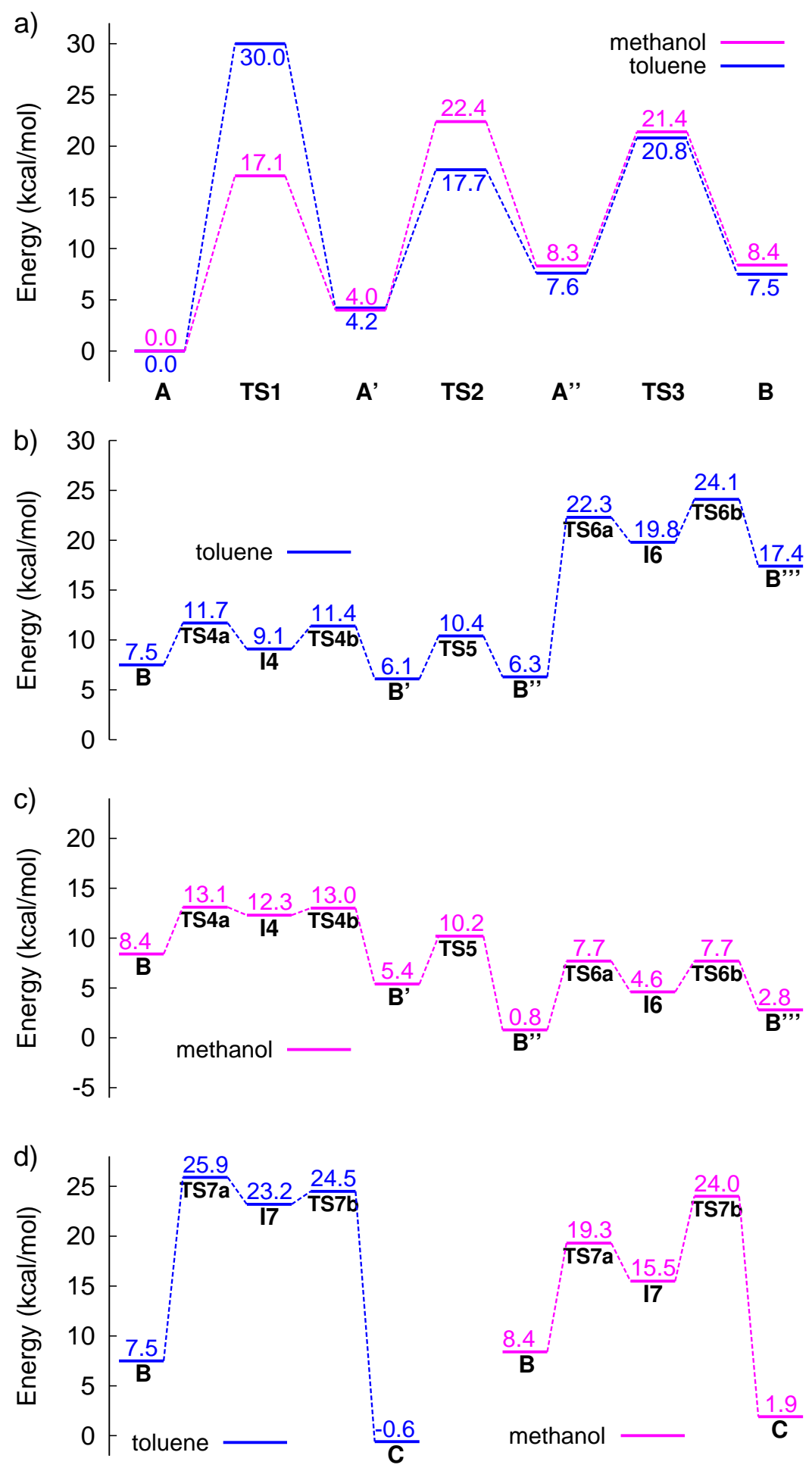

Figure 4.8: Thermal step of DASA-2 in toluene (blue) and methanol (purple) for (a) the ring closure reaction, (b) and (c) tautomerization to the zwitterionic $\mathbf{B}^{\prime \prime \prime}$ product, and (d) the enol-keto tautomerization leading to $\mathbf{C}$. 


\subsubsection{Thermal step of DASA-2}

Second-generation DASA molecules have a more extended $\pi$ conjugation as compared to their first-generation counterpart thanks to the presence of an aniline-based donor (see Figure 4.2). The primary motivation for the design of second-generation DASAs is to increase the wavelength of absorption and, consequently, the penetration depth in (bio)materials $[3,4]$. This strategy, however, is found to introduce a drawback, namely, a slower rate of backward switching, for the majority of aniline-based DASAs except a small subset which also includes the DASA-2 molecule investigated in our work. The synthetic procedure to obtain our DASA-2 molecule does not guarantee the stereochemistry of the $\mathrm{C}-\mathrm{N}$ bond connecting the triene and the donor systems [3], but our calculation reveals that the $E$ conformation of the $\mathrm{C}-\mathrm{N}$ bond is preferred by about $4.0 \mathrm{kcal} / \mathrm{mol}$ over the $Z$ conformation. Therefore, we only discuss below the results for the $E$ conformation.

We compute here the two thermal paths also investigated for DASA-1, which lead to the different $\mathbf{B}^{\prime \prime \prime}$ and $\mathbf{C}$ products. As shown in Figure 4.8, we find that, in DASA2 , the ring-closure reaction, $\mathbf{A}^{\prime} \rightarrow \mathbf{B}$, common to both paths has a similar energy profile and the same rate-limiting step in each solvent as in DASA-1, implying again a pivotal role of the solvent in tuning the kinetics. In methanol, the overall thermal barrier in DASA-2 is somewhat lower by $1.8 \mathrm{kcal} / \mathrm{mol}$ that the value in DASA-1, possibly leading to a reduced irradiation time needed for full photobleaching.

For the forking tautomerization pathways, we find that the final zwitterionic product $\mathbf{B}^{\prime \prime \prime}$ is destabilized by about $6.0 \mathrm{kcal} / \mathrm{mol}$ in both solvents with respect to DASA-1, while the enol-keto tautomerization product $\mathbf{C}$ is stabilized by 2.4 and $3.3 \mathrm{kcal} / \mathrm{mol}$ in toluene and methanol, respectively. The destabilization of $\mathbf{B}^{\prime \prime \prime}$ is related to a decrease in nitrogen basicity when going from the methylamine donor in DASA-1 to the 5-methoxyindoline in DASA-2. The destabilization/stabilization of $\mathbf{B}^{\prime \prime \prime} / \mathbf{C}$ leads to an increased importance of the enol-keto tautomerization pathway leading to $\mathbf{C}$ in DASA-2. More specifically, for DASA-2 in toluene, the overall barrier and the reaction energy to obtain $\mathbf{C}$ is lower by 1.8 and $18.0 \mathrm{kcal} / \mathrm{mol}$, respectively, than the corresponding values for the reaction to obtain $\mathbf{B}^{\prime \prime \prime}$. Therefore, both thermodynamic and kinetic aspects of the thermal reaction in toluene favor the formation of $\mathbf{C}$ as the primary final product. In methanol, while the final products of the two tautomerization pathways are similar in energy, the overall barrier to form $\mathbf{C}$ is higher by $10.9 \mathrm{kcal} / \mathrm{mol}$. Therefore, at ambient conditions, $\mathbf{B}^{\prime \prime \prime}$ is the most favored final product while, at higher temperature, the two tautomerization pathways become equally important since the $\mathbf{B}^{\prime \prime \prime}$ and $\mathbf{C}$ are energetically similar. Hence, the route to $\mathbf{C}$ in methanol becomes increasingly important at higher temperature, in contrast to the DASA-1 case where both thermodynamic and kinetic considerations strongly favor the formation of $\mathbf{B}^{\prime \prime \prime}$ in this polar solvent.

Finally, we note that, in methanol, all zwitterionic intermediates in the complete reaction $\mathbf{A} \rightarrow \mathbf{B}^{\prime \prime \prime}$ increase their energies with respect to the DASA-1 case to such an extent that the overall photoswitching reaction becomes energetically reversible in DASA-2, while the switching in DASA-1 is irreversible. 


\subsubsection{Actinic step}

We simplify the DASA molecule for our excited-state study by substituting two methyl groups on the Meldrum acid moiety with two hydrogen atoms as illustrated in Figure 4.9. This modification will not affect the fundamental features of the groundand excited-state potential energy surfaces since the place of substitution is far from the conjugated stretch of the molecule. The minimal complete active space for the conjugated system is a CAS $(12,11)$ where we correlate 12 electrons in 11 orbitals consisting of the $\pi$ lone pair of the nitrogen atom, three $\pi$ and three $\pi^{*}$ orbitals of the triene, and two $\pi$ and two $\pi^{*}$ orbitals of the carbonyl groups. A larger CAS $(14,13)$ active space was recently employed for a similar diethylamine Meldrum DASA compound [16], including an additional $\sigma$ and $\sigma^{*}$ orbitals on the diethyl branches. We will however not consider such active space because these $\sigma$ and $\sigma^{*}$ orbitals are not expected to play a role in the study of the $\pi \rightarrow \pi^{*}$ excited state. We tested instead the performance of the smaller CAS $(10,9)$ and CAS $(8,7)$ active spaces to establish whether a computational saving could be attained with no sacrifice in accuracy. The CAS $(10,9)$ active space is obtained by eliminating the $\pi$ and $\pi^{*}$ orbitals on the $\mathrm{C}_{10}=\mathrm{O}_{11}$ carbonyl group, which does not participate in the intramolecular hydrogen bond with the alcohol group. For the CAS $(8,7)$, we discard the $\pi$ and $\pi^{*}$ orbitals of the two carbonyl groups, i.e. $\mathrm{C}_{10}=\mathrm{O}_{11}$ and $\mathrm{C}_{8}=\mathrm{O}_{9}$.

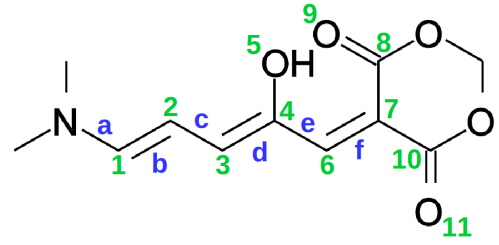

A

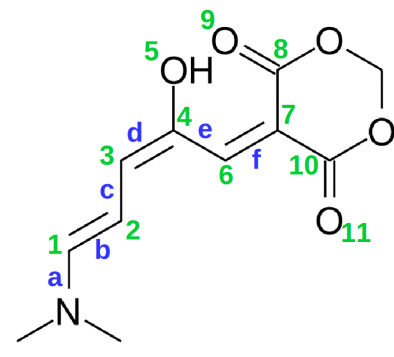

$\mathbf{A}^{\prime}$

Figure 4.9: $\mathbf{A}$ and $\mathbf{A}^{\prime}$ forms of the dimethylamine Meldrum DASA model obtained by substituting two methyl groups on the Meldrum moiety with two hydrogens. Atoms are numbered in green, and conjugated bonds in the linear triene are labeled in blue.

With these three active spaces, we compute the vertical excitation energies of A at the B3LYP/6-31G* ground-state geometry with different numbers of states in the state-average CASSCF and multi-state CASPT2 procedures, and summarize the results in Table 4.1. In all cases, the $\mathrm{S} 1$ state is the bright state with a CASSCF oscillator strength of at least 1.3 a.u. in agreement with the sole presence of one broad peak in the visible absorption spectrum [1]. We find that different CAS expansions yield rather equivalent vertical excitation energies of the S1 state both at the CASSCF and the CASPT2 level. Furthermore, the higher S2 and S3 dark states are blue-shifted by at least $0.9 \mathrm{eV}$ with respect to $\mathrm{S} 1$ and will not participate in the initial relaxation of the bright state. Therefore, all calculations below are performed using only two states. 
Table 4.1: CASSCF and CASPT2 vertical excitation energies (eV) of the $\mathbf{A}$ isomer model computed at the B3LYP/6-31G* ground-state geometry with different active spaces and number of states in the state-average calculations.

\begin{tabular}{|c|c|c|c|c|c|c|c|c|c|}
\hline & \multicolumn{3}{|c|}{ CAS $(8,7)$} & \multicolumn{3}{|c|}{ CAS $(10,9)$} & \multicolumn{3}{|c|}{ CAS $(12,11)$} \\
\hline & $\mathrm{S} 1$ & $\mathrm{~S} 2$ & $\mathrm{~S} 3$ & S1 & S2 & S3 & S1 & $\mathrm{S} 2$ & S3 \\
\hline \multicolumn{10}{|l|}{ CASSCF } \\
\hline SA2 & 3.23 & & & 3.27 & & & 3.30 & & \\
\hline SA3 & 3.35 & 4.54 & & 3.43 & 4.49 & & 3.48 & 4.41 & \\
\hline SA4 & 3.47 & 4.49 & 5.69 & 3.54 & 4.44 & 5.66 & 3.59 & 4.36 & 5.61 \\
\hline \multicolumn{10}{|l|}{ CASPT2 } \\
\hline MS2 & 2.87 & & & 2.87 & & & 2.89 & & \\
\hline MS3 & 2.92 & 4.18 & & 2.94 & 4.32 & & 2.96 & 4.35 & \\
\hline MS4 & 2.94 & 4.32 & 5.49 & 2.96 & 4.37 & 5.55 & 2.98 & 4.39 & 5.57 \\
\hline
\end{tabular}

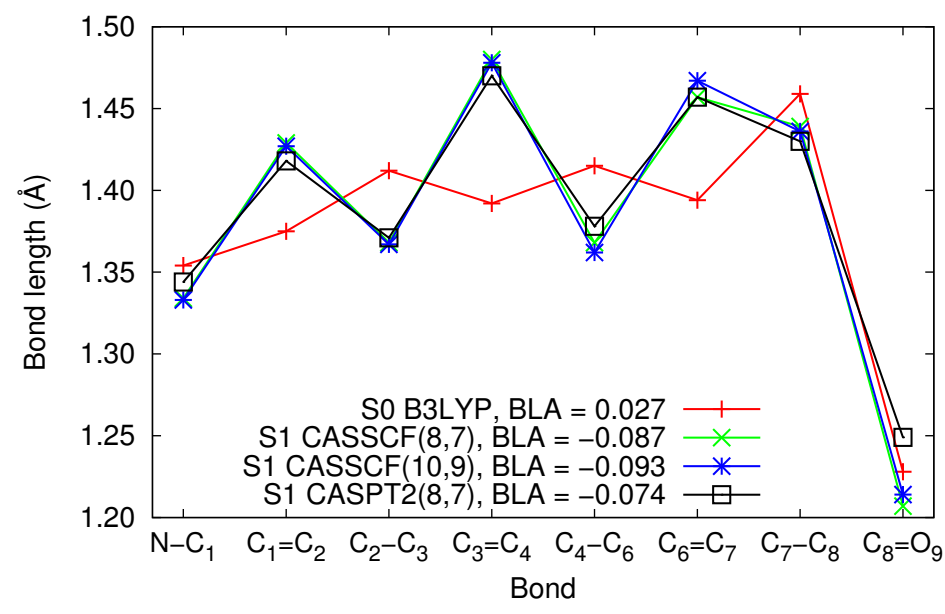

Figure 4.10: Bond lengths of the ground-state B3LYP/6-31G* geometry and the excited-state optimal geometries at the CASSCF and CASPT2 levels.

To investigate the potential energy surface outside the Franck-Condon region, we begin by optimizing the excited-state geometry without any planarity constraint and report the resulting bond lengths in Figure 4.10. With the smaller CAS $(8,7)$ and CAS $(10,9)$ active spaces, we find a slightly distorted in-plane minimum at the CASSCF level. These optimal geometries display a strong inversion of the bond pattern, with a BLA of -0.087 and $-0.093 \AA$ for the CAS $(8,7)$ and $\operatorname{CAS}(10,9)$ expansions, respectively. However, by enlarging the active space to a CAS $(12,11)$, we no longer obtain an excited-state minimum in CASSCF even when we start the optimization from the bond-inverted geometries obtained with the smaller active spaces. The CASSCF $(12,11)$ excited-state geometry optimization proceeds instead toward a conical intersection with a rotation around the dihedral angle $\Phi_{f}$. The preference to rotate around $\Phi_{f}$ can be foreseen from the trend of results obtained with the smaller active spaces: While other dihedral angles remain largely unchanged, $\Phi_{f}$ increases 
from $-14.4^{\circ}$ to $-23.4^{\circ}$ going from a $\operatorname{CAS}(8,7)$ to a $\operatorname{CAS}(10,9)$. Since these reduced spaces lead to different results from the minimal CAS $(12,11)$, we will not further consider them below.

To further understand the energy profile of the CASSCF $(12,11)$ wave function, we compute the MEP of the S1 state starting from the ground-state geometry. The geometrical parameters and energies along the MEP are shown in Figure 4.11. We find that, at the hypersphere radius of 0.05 a.u., the bond-length pattern in the excited state is already inverted, and the excited-state energy is lowered by $0.5 \mathrm{eV}$. Starting from a radius of 0.25 a.u., the $\Phi_{f}$ dihedral angle increases monotonically, and the CASSCF path proceeds without a barrier towards the conical interaction region via a rotation around $\Phi_{f}$. When the excited-state geometry reaches a radius of 0.8 a.u., the BLA begins to increase, and the $\mathrm{O}_{9}-\mathrm{H}$ bond gradually weakens until it ultimately breaks when the state approaches the conical intersection region. Interestingly, the change in $\Phi_{f}$ is not accompanied by a change of the neighboring dihedral angle, $\Phi_{e}$, but the effect is propagated to the next dihedral angle, $\Phi_{d}$, which changes by about $20^{\circ}$ in the vicinity of the conical intersection. There is no proton transfer between $\mathrm{O}_{5}$ and $\mathrm{O}_{9}$ as the proton is always covalently attached to the $\mathrm{O}_{5}$ atom along the whole path.

The barrierless rotation around $\Phi_{f}$ obtained at the $\operatorname{CASSCF}(12,11)$ is unphysical. This rotation transforms the excited state of $\mathbf{A}$ to the ground state of the same form, and consequently, the photoisomerization would never proceed to the closed isomers observed experimentally. Moreover, such a rotation is counterintuitive since it is accompanied by the breaking of an intramolecular hydrogen bond which requires an activation energy. To elucidate the "real" consequences of a $\Phi_{f}$ rotation, we also perform single-point CASPT2 $(12,11)$ calculations on the $\operatorname{CASSCF}(12,11) \mathrm{MEP}$ geometries and show the results in Figure 4.11a. We find that CASPT2//CASSCF gives a barrier of $0.29 \mathrm{eV}$ before reaching the conical intersection region, which can be partially related to the energy cost of breaking a hydrogen bond. In principle, one can obtain a better CASSCF description by further expanding the active space to include appropriate bonding and antibonding orbitals beyond the minimal CAS $(12,11)$ choice. However, one then faces the problem that larger active spaces become computationally very demanding, and different expansions might be needed in different regions of the excited-state PES.

Here, we will attempt to include the missing electron correlation by performing CASPT2 geometry optimization and employ a medium-size CAS $(8,7)$ active space which covers the whole triene stretch. Surprisingly, we find a near in-plane minimum at the CASPT2 level as in the $\operatorname{CASSCF}(8,7)$ and $\operatorname{CASSCF}(10,9)$ calculations, with only a somewhat less pronounced inversion of the bond-length pattern (BLA of $-0.074 \AA$ ) as shown in Figure 4.10. The presence of a minimum in the excited-state PES indicates that there is a barrier of rotation around all formal double bonds. The question of course arises as to whether CASPT2 with a CAS $(8,7)$ active space is able to improve upon the corresponding $\operatorname{CASSCF}(8,7)$ calculation. Since we know that CASSCF and CASPT2//CASSCF with the larger CAS $(12,11)$ lead to qualitatively different energy profiles, further CASPT2 constrained geometry optimizations or MEP computations with larger active spaces are required to accurately explore the 

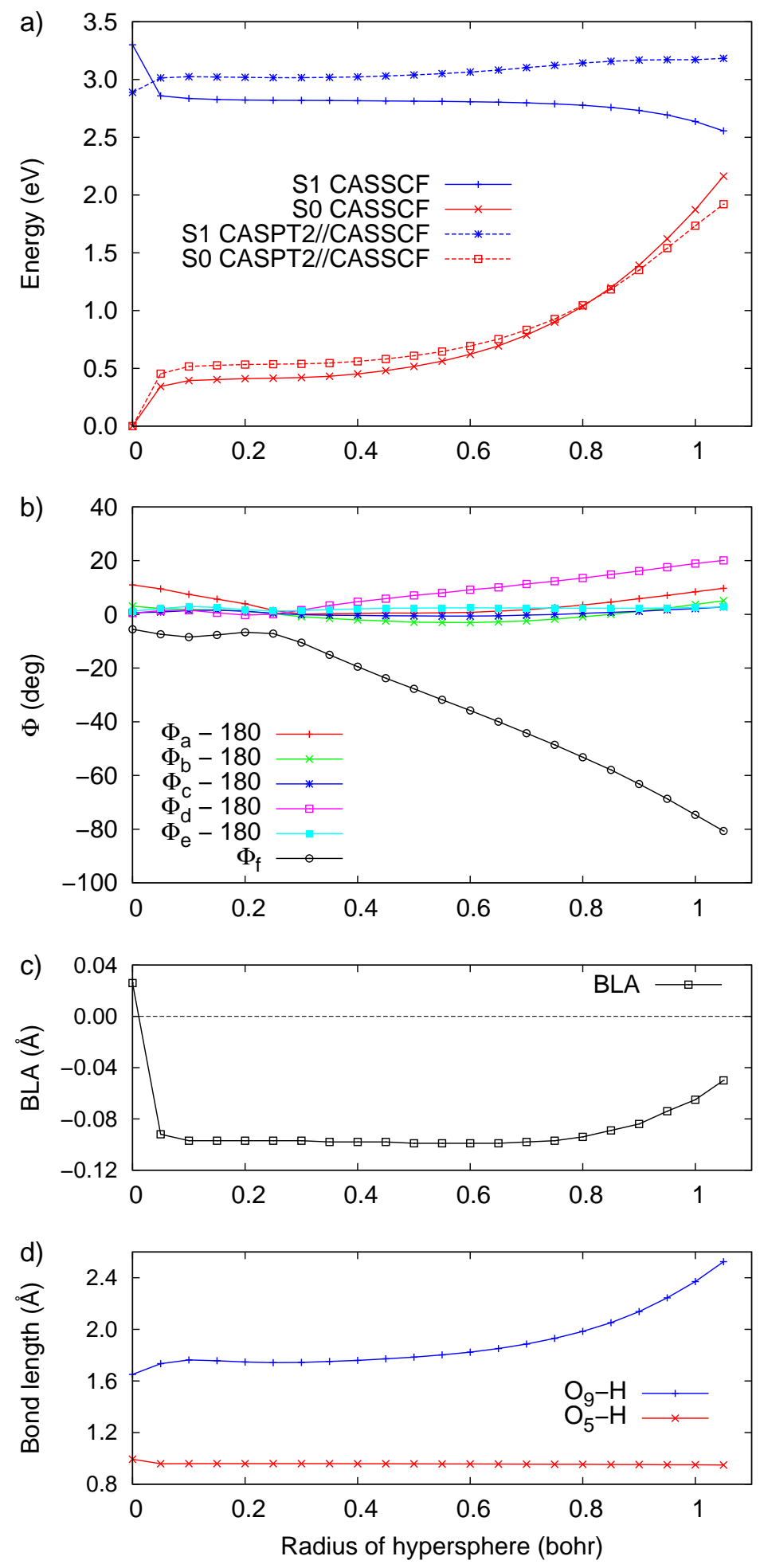

Figure 4.11: Minimum energy path of the S1 state of the $\mathbf{A}$ isomer model. (a) S0 and S1 energies at the CASSCF and CASPT2//CASSCF level, (b) evolution of the dihedral angles, (c) BLA and (d) the $\mathrm{O}-\mathrm{H}$ bonds. 
excited-state PES of DASA.
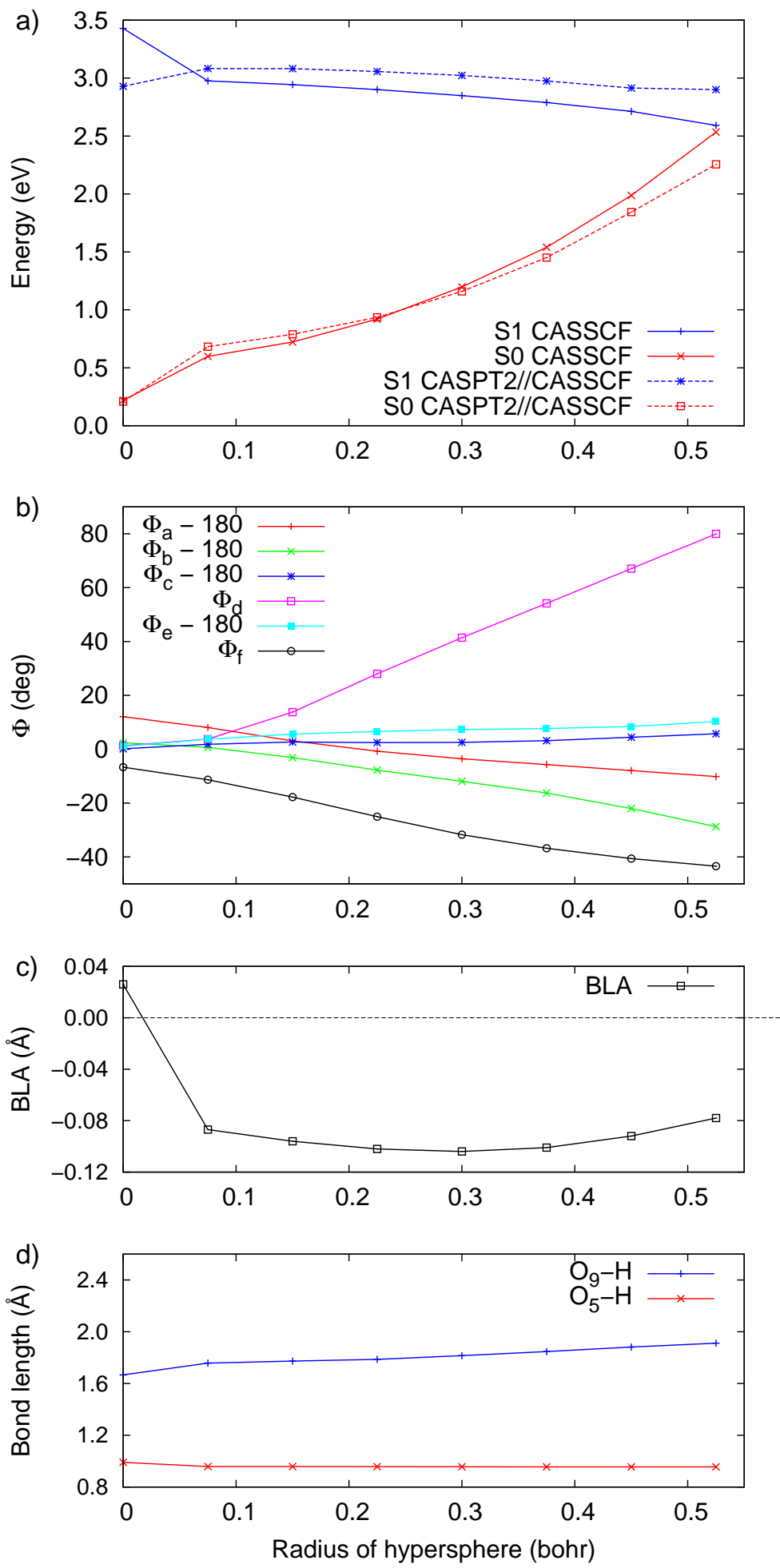

Figure 4.12: Minimum energy path of the S1 state of the $\mathbf{A}^{\prime}$ isomer model. (a) S0 and S1 energies at the CASSCF and CASPT2//CASSCF level, (b) evolution of the dihedral angles, (c) BLA and (d) the $\mathrm{O}-\mathrm{H}$ bonds. 
Finally, motivated by the experimental observation that the excited state of the $\mathbf{A}^{\prime}$ isomer can relax to the ground state of $\mathbf{A}$, we calculate the $\operatorname{CASSCF}(12,11)$ excitedstate MEP starting from the ground-state B3LYP/6-31G* geometry of $\mathbf{A}^{\prime}$ and present the results in Figure 4.12. We find that the relaxation proceeds by first inverting the bond pattern, resulting in a negative BLA value. The excited-state then relaxes without a barrier towards a conical intersection region via a rotation around $\Phi_{d}$, which can ultimately transform $\mathbf{A}^{\prime}$ into $\mathbf{A}$. Along the path, the hydrogen is always covalently attached to the $\mathrm{O}_{5}$ atom, and the hydrogen bond to $\mathrm{O}_{9}$ is found to weaken as the hypersphere radius increases. Other dihedral angles for formal double bonds $\left(\Phi_{b}\right.$ and $\Phi_{f}$ ) are also substantially affected as a result of the $\Phi_{d}$ rotation, which indicates that other relaxation paths might become active at ambient conditions. The energy refinement through the CASPT2//CASSCF method gives however a different picture, yielding a barrier of $0.15 \mathrm{eV}$ along this path and demonstrating again the importance of including dynamical electron correlation in the description of the excited-state PES of DASA. Therefore, although the CASSCF $(12,11)$ excited-state MEP of $\mathbf{A}^{\prime}$ indicates the preference for $\Phi_{d}$ rotation which is in line with experimental results, further excited-state pathway computations at a higher correlated level are highly desirable due to the clear inadequacy of the commonly used CASSCF approach.

\subsection{Conclusions}

In summary, we have elucidated all reaction steps of the thermal isomerization pathways of two photoswitches belonging to the first- and second-generation DASA family in different solvents. Starting from the final product of the actinic step $\mathbf{A}^{\prime}$, a thermally-allowed single-bond rotation takes place to form $\mathbf{A}^{\prime \prime}$, which is followed by a one-step conrotary $4 \pi$ electrocyclization resulting in a closed $\mathbf{B}$ form. Afterwards, tautomerization can occur along two distinct routes without the direct involvement of the solvent. The first route proceeds in three consecutive steps which bring the hydrogen from the $\mathrm{OH}$ group of $\mathbf{B}$ to the amine group of the final zwitterionic $\mathbf{B}^{\prime \prime \prime}$ isomer. The second route involves an intramolecular enol-keto tautomerization resulting in a neutral diketo product, $\mathbf{C}$.

In DASA-1, the forward switching is irreversible in methanol due to the stabilization of the zwitterionic $\mathbf{B}^{\prime \prime \prime}$. In contrast, photoswitching is reversible in toluene and the two tautomerization routes are differently regulated: the first route is kinetically controlled while the second one will be more likely to occur at higher temperature, being therefore a thermodynamically-controlled reaction.

In DASA-2, we observe the destabilization of $\mathbf{B}^{\prime \prime \prime}$ and the stabilization of $\mathbf{C}$, which correlate to the experimental observation that $\mathbf{C}$ is the major end product. In toluene, the two tautomerization routes are kinetically similar but thermodynamically different with the $\mathbf{C}$ end product being energetically more stable by $18.0 \mathrm{kcal} / \mathrm{mol}$ than $\mathbf{B}^{\prime \prime \prime}$. In methanol, the first route is more likely to occur at low temperatures while the second route will be equally visited at higher temperature. Moreover, the whole reaction path $\mathbf{A} \rightarrow \mathbf{B}^{\prime \prime \prime}$ becomes reversible as the energies of all intermediates after the closed $\mathbf{B}$ form are higher than the initial $\mathbf{A}$. 
Our preliminary work on the excited-state relaxation of the initial isomer indicates that the CASSCF approach is inadequate to explain experimental observations. The CASSCF minimum energy path of the $\mathbf{A}$ isomer proceeds without a barrier towards the conical intersection via rotation of the Meldrum moiety, which breaks a hydrogen bond. Beyond the conical intersection, the reaction would return to the initial A form. Therefore, not only the CASSCF method gives the incorrect picture that the end product of photoswitching is the initial isomer, but it also predicts isomerization around the only formal double bond which is constrained by an intramolecular hydrogen bond. Importantly, the CASPT2//CASSCF method indicates the existence of a barrier along the CASSCF path. Surprisingly, CASPT2 geometry optimization with a medium-size CAS $(8,7)$ active space finds a slightly distorted in-plane minimum and, consequently, a barrier to reach the conical intersection region. Further study at the CASPT2 level with a larger active space or with other correlated approaches are needed to understand what appears to be a rather complex excited-state potential energy surface, whose description requires a balanced treatment of static and dynamical correlation. 


\subsection{Appendix: Other functionals}

The M06-2X functional explains very well many experimental observations in both the DASA-1 and the DASA-2 molecule. Here, we focus on DASA-1 and compare the performance of two other functionals which are also frequently used to calculate ground-state potential energy surfaces, namely $\omega$ B97X-D and B3LYP. As shown in Table 4.2, the $\omega \mathrm{B} 97 \mathrm{X}-\mathrm{D}$ functional gives essentially the same potential energy paths as the M06-2X functional, although the relative energies of all minima and transition states are shifted downward.

The B3LYP functional yields a similar energy profile to the M06-2X functional for the reaction between open conformers and for the tautomerization steps. However, the ring-closure $\mathbf{A}^{\prime \prime} \rightarrow \mathbf{B}$ step is very different: the B3LYP barrier is higher by 6.1 and $7.0 \mathrm{kcal} / \mathrm{mol}$, and the reaction energy is greater by 13.0 and $12.0 \mathrm{kcal} / \mathrm{mol}$ in toluene and methanol, respectively. Hence, all closed isomers are energetically too high in B3LYP and, consequently, the thermal step is reversible in methanol, a finding which is in contradiction with experimental observations. Even though the potential energy profile is not consistent with experiments, the B3LYP functional could still be useful for DASAs' investigations since it gives a very good estimate of the infrared spectra [13]. 
Table 4.2: Energies of minima and transition states of DASA-1 computed with the $\omega$ B97X-D and B3LYP functionals and the maug-cc-pVDZ basis set. Numbers in parentheses indicate the difference with respect to the corresponding M06-2X value.

\begin{tabular}{|c|c|c|c|c|}
\hline \multirow[t]{2}{*}{ Isomer } & \multicolumn{2}{|c|}{$\omega B 97 X-D$} & \multicolumn{2}{|c|}{ B3LYP } \\
\hline & Toluene & Methanol & Toluene & Methanol \\
\hline \multicolumn{5}{|c|}{ Ring closure } \\
\hline $\mathbf{A}$ & $0.0(0.0)$ & $0.0(0.0)$ & $0.0(0.0)$ & $0.0(0.0)$ \\
\hline TS1 & $28.4(-0.7)$ & $15.4(-1.2)$ & $32.1(3.0)$ & $20.0(3.4)$ \\
\hline $\mathbf{A}^{\prime}$ & $3.6(0.1)$ & $2.7(-1.6)$ & $4.0(0.5)$ & $3.9(-0.4)$ \\
\hline TS2 & $16.3(-1.1)$ & $22.6(-1.9)$ & $21.0(3.6)$ & $26.7(2.2)$ \\
\hline $\mathbf{A}^{\prime \prime}$ & $7.0(-1.1)$ & $6.8(-1.2)$ & $9.0(0.9)$ & $9.5(1.5)$ \\
\hline TS3 & $19.9(-0.4)$ & $21.0(-1.9)$ & $27.3(7.0)$ & $31.4(8.5)$ \\
\hline B & $3.9(-2.9)$ & $7.5(-3.0)$ & $20.7(13.9)$ & $24.0(13.5)$ \\
\hline average & $(-0.9)$ & $(-1.5)$ & $(4.1)$ & $(4.1)$ \\
\hline \multicolumn{5}{|c|}{ Tautomerization from $\mathbf{B}$ to $\mathbf{B}^{\prime \prime \prime}$} \\
\hline B & $3.9(-2.9)$ & $7.5(-3.0)$ & $20.7(13.9)$ & $24.0(13.5)$ \\
\hline TS4a & $9.5(-2.4)$ & $14.0(-2.6)$ & $25.2(13.3)$ & $29.8(13.2)$ \\
\hline I4 & $5.3(-2.8)$ & $10.5(-2.7)$ & $21.2(13.1)$ & $25.9(12.7)$ \\
\hline TS4b & $8.6(-2.2)$ & $11.5(-2.4)$ & $25.1(14.3)$ & $27.5(13.6)$ \\
\hline $\mathbf{B}^{\prime}$ & $6.4(-2.3)$ & $6.6(-2.8)$ & $22.8(14.1)$ & $23.5(14.1)$ \\
\hline TS5 & $10.4(-2.7)$ & $11.0(-3.7)$ & $25.8(12.7)$ & $26.9(12.2)$ \\
\hline $\mathbf{B}^{\prime \prime}$ & $1.3(-2.5)$ & $-8.3(-3.9)$ & $17.6(13.8)$ & $8.5(12.9)$ \\
\hline TS6a & $10.7(-3.3)$ & $-3.2(-4.2)$ & $27.2(13.2)$ & $12.6(11.6)$ \\
\hline I6 & $8.5(-3.2)$ & $-6.4(-3.6)$ & $25.0(13.3)$ & $9.9(12.7)$ \\
\hline TS6b & $13.8(-3.5)$ & $-1.4(-3.9)$ & $29.8(12.5)$ & $14.2(11.7)$ \\
\hline $\mathbf{B}^{\prime \prime \prime}$ & $7.9(-3.7)$ & $-7.4(-4.0)$ & $25.8(14.2)$ & $9.9(13.3)$ \\
\hline average & $(-2.9)$ & $(-3.3)$ & (13.5) & (12.9) \\
\hline \multicolumn{5}{|c|}{ Tautomerization from $\mathbf{B}$ to $\mathbf{C}$} \\
\hline B & $3.9(-2.9)$ & $7.5(-3.0)$ & $20.7(13.9)$ & $24.0(13.5)$ \\
\hline TS7a & $20.6(-3.4)$ & $17.8(-8.4)$ & $35.8(11.8)$ & $33.1(6.9)$ \\
\hline $\mathbf{I 7}$ & $17.4(-4.3)$ & $12.5(-3.3)$ & $33.1(11.4)$ & $28.4(12.6)$ \\
\hline TS7b & $19.5(-3.9)$ & $20.6(-6.5)$ & $35.6(12.2)$ & $36.5(9.4)$ \\
\hline C & $-3.2(-5.0)$ & $-0.8(-6.0)$ & $15.2(13.4)$ & 16.8 (11.6) \\
\hline average & $(-3.9)$ & $(-5.4)$ & (12.5) & (10.8) \\
\hline
\end{tabular}


4 Photo-induced mechanism of isomerization of DASA 


\section{Bibliography}

[1] S. Helmy, F. A. Leibfarth, S. Oh, J. E. Poelma, C. J. Hawker, and J. Read de Alaniz, J. Am. Chem. Soc. 136, 8169 (2014).

[2] S. Helmy, S. Oh, F. A. Leibfarth, C. J. Hawker, and J. Read de Alaniz, J. Org. Chem. 79, 11316 (2014).

[3] J. R. Hemmer, S. O. Poelma, N. Treat, Z. A. Page, N. D. Dolinski, Y. J. Diaz, W. Tomlinson, K. D. Clark, J. P. Hooper, C. J. Hawker, and J. Read de Alaniz, J. Am. Chem. Soc. 138, 13960 (2016).

[4] N. Mallo, P. T. Brown, H. Iranmanesh, T. S. MacDonald, M. J. Teusner, J. B. Harper, G. E. Ball, and J. E. Beves, Chem. Commun. 52, 13576 (2016).

[5] S. Singh, K. Friedel, M. Himmerlich, Y. Lei, G. Schlingloff, and A. Schober, ACS Macro Lett. 4, 1273 (2015).

[6] A. Balamurugan and H.-i. Lee, Macromolecules 49, 2568 (2016).

[7] G. Sinawang, B. Wu, J. Wang, S. Li, and Y. He, Macromol. Chem. Phys. 217, 2409 (2016).

[8] B. Mason, M. Whittaker, J. Hemmer, S. Arora, A. Harper, S. Alnemrat, A. McEachen, S. Helmy, J. Read de Alaniz, and J. Hooper, Appl. Phys. Lett. 108, 041906 (2016).

[9] S. Jia, J. D. Du, A. Hawley, W.-K. Fong, B. Graham, and B. J. Boyd, Langmuir 33, 2215 (2017).

[10] F.-Y. Tang, J.-N. Hou, K.-X. Liang, Y. Liu, L. Deng, and Y.-N. Liu, New J. Chem. 41, 6071 (2017).

[11] S. O. Poelma, S. S. Oh, S. Helmy, A. S. Knight, G. L. Burnett, H. T. Soh, C. J. Hawker, and J. R. de Alaniz, Chem. Commun. 52, 10525 (2016).

[12] M. M. Lerch, S. J. Wezenberg, W. Szymański, and B. L. Feringa, J. Am. Chem. Soc. 138, 6344 (2016).

[13] M. Di Donato, M. M. Lerch, A. Lapini, A. D. Laurent, A. Iagatti, L. Bussotti, S. P. Ihrig, M. Medved', D. Jacquemin, W. Szymański, W. J. Buma, P. Foggi, and B. L. Feringa, J. Am. Chem. Soc. 139, 15596 (2017). 
[14] Private communication with M. M. Lerch and W. Szymański.

[15] A. D. Laurent, M. Medved', and D. Jacquemin, Chem. Phys. Chem. 17, 1846 (2016).

[16] C. García-Iriepa and M. Marazzi, Materials 10, 1025 (2017).

[17] J. N. Bull, E. Carrascosa, N. Mallo, M. S. Scholz, G. da Silva, J. E. Beves, and E. J. Bieske, J. Phys. Chem. Lett. 9, 665 (2018).

[18] Y. Zhao and D. G. Truhlar, Theor. Chem. Acc. 120, 215 (2008).

[19] R. A. Kendall, T. H. Dunning Jr, and R. J. Harrison, J. Chem. Phys. 96, 6796 (1992).

[20] E. Papajak, J. Zheng, X. Xu, H. R. Leverentz, and D. G. Truhlar, J. Chem. Theory Comput. 7, 3027 (2011).

[21] A. V. Marenich, C. J. Cramer, and D. G. Truhlar, J. Phys. Chem. B 113, 6378 (2009).

[22] J.-D. Chai and M. Head-Gordon, Phys. Chem. Chem. Phys. 10, 6615 (2008).

[23] N. Mardirossian and M. Head-Gordon, Mol. Phys. 115, 2315 (2017).

[24] A. D. Becke, Phys. Rev. A. 38, 3098 (1988).

[25] C. Lee, W. Yang, and R. G. Parr, Phys. Rev. B. 37, 785 (1988).

[26] A. D. Becke, J. Chem. Phys. 98, 5648 (1993).

[27] M. J. Frisch, G. W. Trucks, H. B. Schlegel, G. E. Scuseria, M. A. Robb, J. R. Cheeseman, G. Scalmani, V. Barone, B. Mennucci, G. A. Petersson, H. Nakatsuji, M. Caricato, X. Li, H. P. Hratchian, A. F. Izmaylov, J. Bloino, G. Zheng, J. L. Sonnenberg, M. Hada, M. Ehara, K. Toyota, R. Fukuda, J. Hasegawa, M. Ishida, T. Nakajima, Y. Honda, O. Kitao, H. Nakai, T. Vreven, J. A. Montgomery, Jr., J. E. Peralta, F. Ogliaro, M. Bearpark, J. J. Heyd, E. Brothers, K. N. Kudin, V. N. Staroverov, R. Kobayashi, J. Normand, K. Raghavachari, A. Rendell, J. C. Burant, S. S. Iyengar, J. Tomasi, M. Cossi, N. Rega, J. M. Millam, M. Klene, J. E. Knox, J. B. Cross, V. Bakken, C. Adamo, J. Jaramillo, R. Gomperts, R. E. Stratmann, O. Yazyev, A. J. Austin, R. Cammi, C. Pomelli, J. W. Ochterski, R. L. Martin, K. Morokuma, V. G. Zakrzewski, G. A. Voth, P. Salvador, J. J. Dannenberg, S. Dapprich, A. D. Daniels, Ö. Farkas, J. B. Foresman, J. V. Ortiz, J. Cioslowski, and D. J. Fox, Gaussian 09 Revision D.01, gaussian Inc. Wallingford CT 2009.

[28] B. O. Roos, P. R. Taylor, and P. E. Siegbahn, Chem. Phys. 48, 157 (1980).

[29] K. Andersson, P.-Å. Malmqvist, B. O. Roos, A. J. Sadlej, and K. Wolinski, J. Phys. Chem. 94, 5483 (1990). 
[30] K. Andersson, P.- $\AA$. Malmqvist, and B. O. Roos, J. Chem. Phys. 96, 1218 (1992).

[31] J. Finley, P.-Å. Malmqvist, B. O. Roos, and L. Serrano-Andrés, Chem. Phys. Lett. 288, 299 (1998).

[32] J. Stålring, A. Bernhardsson, and R. Lindh, Mol. Phys. 99, 103 (2001).

[33] G. Ghigo, B. O. Roos, and P.-Å. Malmqvist, Chem. Phys. Lett. 396, 142 (2004).

[34] N. Forsberg and P.- $̊$. Malmqvist, Chem. Phys. Lett. 274, 196 (1997).

[35] F. Aquilante, P.-Å. Malmqvist, T. B. Pedersen, A. Ghosh, and B. O. Roos, J. Chem. Theory Comput. 4, 694 (2008).

[36] F. Aquilante, J. Autschbach, R. K. Carlson, L. F. Chibotaru, M. G. Delcey, L. De Vico, I. Fdez. Galván, N. Ferré, L. M. Frutos, L. Gagliardi, M. Garavelli, A. Giussani, C. E. Hoyer, G. Li Manni, H. Lischka, D. Ma, P. Å. Malmqvist, T. Müller, A. Nenov, M. Olivucci, T. B. Pedersen, D. Peng, F. Plasser, B. Pritchard, M. Reiher, I. Rivalta, I. Schapiro, J. Segarra-Martí, M. Stenrup, D. G. Truhlar, L. Ungur, A. Valentini, S. Vancoillie, V. Veryazov, V. P. Vysotskiy, O. Weingart, F. Zapata, and R. Lindh, J. Comput. Chem. 37, 506 (2016).

[37] D. R. Wenz and J. Read de Alaniz, Eur. J. Org. Chem. 2015, 23 (2015).

[38] G. Piancatelli, A. Scettri, and S. Barbadoro, Tetrahedron Lett. 17, 3555 (1976).

[39] O. Nieto Faza, C. Silva López, R. Álvarez, and Á. R. de Lera, Chem. Eur. J. 10, 4324 (2004).

[40] S.-W. Li and R. A. Batey, Chem. Commun. 3759 (2007). 


\section{Chapter 5}

\section{Regulating allosteric interactions in a protein hybrid with light: Mechanistic insight from molecular dynamics ${ }^{\dagger}$}

The development of strategies to interfere with allosteric interactions in proteins is of importance not only to deepen our understanding of vital cellular processes but also to enable their regulation using external triggers. Light is particularly attractive for this purpose since it is spatiotemporally selective and applicable in physiological environments. Our experimental collaborators have recently engineered a photo-responsive hybrid protein and established what seemed to be a new allosteric communication route which is not inherent to the natural system. The human serum albumin was selected for this purpose due to its promiscuous behavior and importance in transporting a variety of ligands in plasma. By covalently incorporating a synthetic photoswitch at subdomain IA, optical control was experimentally achieved on ligand binding at the distinct subdomain IB. Our molecular dynamics simulations confirm the allosteric nature of the interactions between IA and IB, and reveal that the presence of the switch at IA destabilizes a key hydrogen bond of the ligand with the IB subdomain. Photo-conversion is found to correlate with a less coordinated motion of the two subdomains and an increased flexibility of the binding pocket, whose fluctuations are cooperatively enhanced by the presence of the ligands, ultimately facilitating their release. Our combined experimental and computational work demonstrate how harnessing artificial molecular switch enables photo-programming the allosteric regulation of binding activities in such a prominent protein.

\footnotetext{
${ }^{\dagger}$ This chapter with some additional experimental work is accepted on Bioconjug. Chem. as R. M. Putri, H. Zulfikri, J. W. Fredy, A. Juan, P. Tananchayakul, J. J. L. M. Cornelissen, M. S. T. Koay, C. Filippi, and N. Katsonis "Photo-programming allostery in the human serum albumin". The first two authors contributed equally to this publication.
} 


\subsection{Introduction}

In proteins with multiple binding pockets, binding of a ligand at one site often influences the binding of ligands to other remote binding sites, a process also known as allostery [1-5]. While allosteric regulation of protein activity is central to many biological processes, the molecular mechanisms by which proteins undergo allosteric regulation remain largely unknown and allosteric ligand binding has already revealed a number of paradoxes. For example, tightly packed, fully folded proteins can display a remarkable structural plasticity dictated by allosteric communication among binding pockets. On the other hand, a structural change in the protein backbone is not always necessary for the binding sites to communicate with each other [4]. Overall, evidence is growing that allostery is facilitated in dynamic proteins and is often not simply a shape change-induced phenomenon as understood in earlier years [6], but rather a statistical process in an ensemble of conformational states of the ligandprotein system [1-4]. Pioneering work has also highlighted the limitations of deducing mechanisms from the static equilibrium structures of the end states alone $[1,3]$.

The state of the art thus calls for tools to manipulate, investigate and eventually harness the dynamics of allosteric systems. A recent study reports on the use of temperature and $\mathrm{pH}$ control to increase the local conformational entropy of fused proteins that communicate through allostery [7]. A parallel effort towards allosteric engineering consists in implementing allosteric behavior in wholly synthetic biomimetic systems to control ligand binding and catalysis $[8,9]$. However, previous approaches lack selectivity in space and time, which is a limitation for protein systems operating at physiological conditions, because environmental changes for instance in $\mathrm{pH}$ or temperature can lead to unfolding and loss of activity.

In contrast, light offers high spatiotemporal control while being potentially nondestructive toward proteins and applicable in physiological conditions [10-14]. Arguably, the most straightforward strategy to make proteins light-switchable is to make use of artificial molecular photoswitches, provided that the stability and the photoswitching properties of the molecular switches are preserved in the protein environment [15]. Light-modulated proteins have been developed to control biological activities, such as directing the secondary structures of peptides/proteins $[16,17]$, enzymatic catalysis $[18,19]$, protein-ligand binding [20,21], modulation of protein channels [22,23], and receptors in neural networks [24]. Recently, the emergence of photoswitchable allosteric modulators [25-27] as well as light-activated allosteric channel and DNA-binding protein $[28,29]$ marks a new era of combining allosteric regulation with tailored optical control, by incorporation of either light-activated protein segments or synthetic molecular switches.

The human serum albumin (HSA) protein constitutes an ideal playground to design novel optical strategies and to modulate specific binding activities. HSA is the most abundant protein in human blood plasma and is the main carrier of a variety of compounds, being able to bind over 120 types of ligands despite its relatively simple monomeric structure [30]. This small protein $(66 \mathrm{kDa})$ is dominated by $\alpha$-helices and loops and characterized by multiple binding sites ( 3 primarily for ligands and 7 for fatty acids), which account for its remarkable ability to bind a broad range of com- 
pounds $[30,31]$. This promiscuity offers an opportunity to modulate a large number of binding events potentially, but it is also associated with a challenge, as the global structural organization of the binding sites in HSA is very flexible, also adapting to the binding of compounds [32] and enabling multiple binding sites to be coupled through allosteric interactions [30]. Recent investigations by ultra-fast time-resolved spectroscopy suggest that these interactions are also associated with ballistic energy flow through the connecting helical structures of HSA [33].

Recently, our experimental collaborators have created a photo-switchable HSA protein by attaching a photoswitch at subdomain IA covalently, and have achieved optical control on ligand release at the distinct binding site of subdomain IB. The effect of photoswitching is therefore propagated between two neighboring subdomains that are covalently connected only through a long flexible coil and do not share any helices. Our extensive molecular dynamics (MD) simulations rule out any possibility of direct interaction between the photoswitch and the binding site of subdomain IB, and reveal that the IB pocket becomes significantly more flexible after photoswitching. In contrast, no enhanced plasticity of subdomain IA is observed, but a reduced coordination in the motion of the two subdomains. This dynamic change correlates to a loss of specific interactions of the ligand with the IB pocket and, ultimately, to the photo-induced release observed experimentally. To the best of our knowledge, allosteric communications between the IA and IB sites have not been reported before. Furthermore, in the absence of the switch, no ligand release is observed upon irradiation. Therefore, our combined theoretical and experimental work suggests that it is possible to leverage on the dynamic nature of allostery and "wire" a communication pathway that is not intrinsic to the natural system, to control the specific binding activity of a protein.

\subsection{Experimental background}

The photo-responsive protein hybrid is constructed by covalently incorporating a photoswitch to a particular sidechain of a monomeric HSA as shown in Figure 5.1a. In HSA, there is only one cysteine residue (Cys34), which is located at subdomain IA and exposed to the solvent, and thus available for chemical modification. The thiol (-SH) group of Cys34 reacts with a maleimide ring bearing a spiropyran (SP) photoswitch in a Tris- $\mathrm{HCl}$ buffer at $\mathrm{pH} 7.5$ containing $20 \%$ dimethylsulfoxide (DMSO) and at room temperature, yielding a HSA-SP hybrid protein according to Michael addition reaction. For the stability of the albumin hybrid, the acidity of the system has to be maintained at a $\mathrm{pH}$ of 7.5 to avoid spontaneous ring opening of SP, which would happen in acidic environment [12], and to avoid the risk of alkaline hydrolysis of the maleimide anchor, which would occur in basic conditions [34].

Spiropyran is chosen as photoswitch because under irradiation with light, it is converted to its open, zwitterionic form, the merocyanine (MC), which is expected to substantially modify electrostatic interactions locally (Figure 5.1b) [12]. Irradiating the HSA-SP hybrid with UV light ( $\lambda=365 \mathrm{~nm}, 5 \mathrm{~min}$ ) triggers the appearance of an absorption band at $\lambda_{\max }=550 \mathrm{~nm}$, which signals the transformation of the colorless 
a)

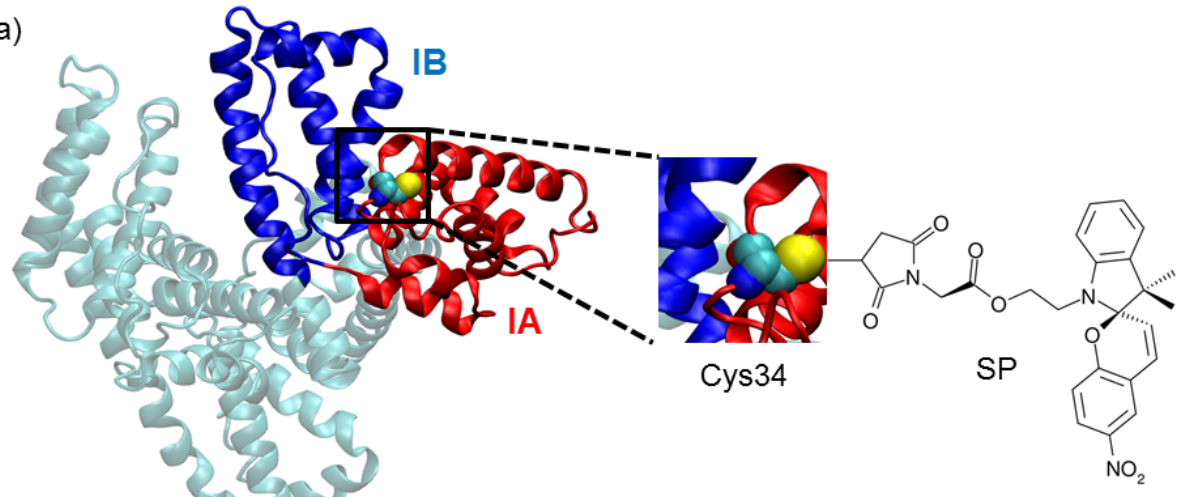

b)

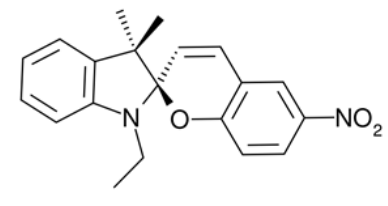

Spiropyran (SP)

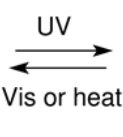

$\underset{\text { Vis or heat }}{\rightleftarrows}$

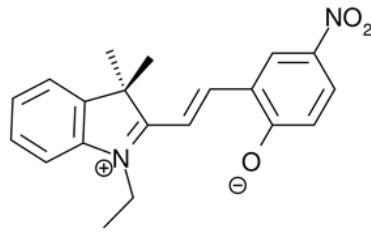

Merocyanine (MC) c)

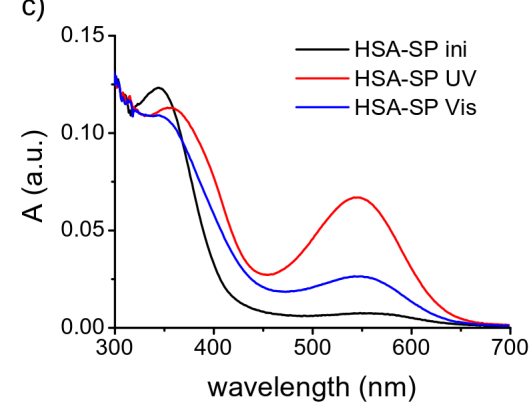

d)

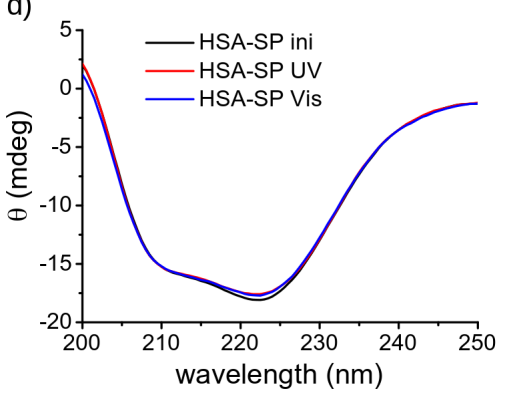

Figure 5.1: Design and synthesis of a photo-switchable hybrid of the human serum albumin (HSA). a) A spiropyran molecule (SP) is connected covalently to HSA via Cys34 in Tris- $\mathrm{HCl}$ buffer $\mathrm{pH}=7.5$ (containing 20\% DMSO). The IA and IB subdomains of HSA are shown in red and blue, respectively. b) Under irradiation with UV light, SP converts into its isomer, merocyanine (MC). c) UV/Vis spectra of the hybrid system before (black) and after irradiation with UV (red) and Vis light (blue). d) CD spectra of the hybrid system before (black) and after irradiation with UV (red) and Vis light (blue).

SP into the colored MC isomer, and demonstrates that the photochromic properties of the switch are preserved after its covalent attachment to the protein (Figure 5.1c). NMR spectroscopy allows determining that the degree of photo-conversion from SP to $\mathrm{MC}$ is around $40 \%$ under the experimental conditions, suggesting that a mixture of HSA-SP and HSA-MC is present in the photo-stationary state. Furthermore, upon irradiation with visible light $(\lambda \geq 420 \mathrm{~nm}, 5 \mathrm{~min})$, the band at $\lambda_{\max }=550 \mathrm{~nm}$ gradually decreases, indicating that some of the MC form switches back to the colorless SP 
form. The effect of irradiation on the global structure of HSA-SP is also analyzed in terms of conformation (with CD spectra) and hydrodynamic size. Both methods do not reveal any significant structural perturbation after irradiation (for the CD spectra, see Figure 5.1d), confirming that the overall structure of the HSA hybrid remains intact in the conditions of the experiment.

The behavior of the albumin hybrid, before and after irradiation with UV light $(\lambda=365 \mathrm{~nm})$, is examined with respect to the binding of the methyl orange (MO) ligand (Figure 5.2) in subdomain IB. For all experiments, an excess of MO ligand ( $\sim 4$ equivalent) is added to HSA-SP and, before each spectrum is measured, the unbound $\mathrm{MO}$ is removed by spin-filtration. From comparing the UV/Vis spectra before and after ligand binding, the protein:ligand ratio is estimated to be 1:1.3. Upon UV irradiation, about $(16 \pm 2) \%$ of the bound MO ligand is released as revealed by the UV/Vis spectra. Reversibility is moderate since only about $(8 \pm 1) \%$ of the released MO binds back after subsequent irradiation with visible light for 5 min. Importantly, in the absence of spiropyran, UV irradiation of the ligand-HSA complex does not yield any significant spectral change. This indicates that MO is stable and not responsive to UV light in the experimental conditions, so any changes upon irradiation is primarily associated to release/binding events.

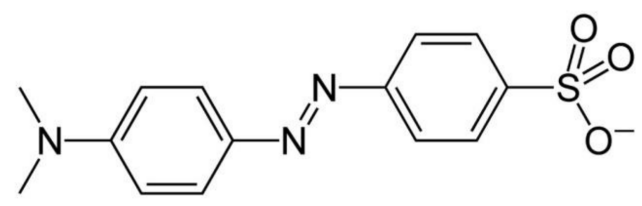

Figure 5.2: Lewis structure of the methyl orange (MO) anion ligand employed in our photo-triggered ligand release and capture study.

Here, we seek to understand the mechanism of photo-controlled ligand release in the albumin hybrid complex. For further details and discussions on the synthesis, characterization, and optical control of the protein hybrid, we refer the reader to the dissertation of our collaborator, Rindia Maharani Putri [35].

\subsection{Computational details}

\subsubsection{Molecular docking}

To generate representative initial configurations of the HSA-SP and HSA-MC systems for our MD simulations, we take the X-ray coordinates of HSA (PDB entry: $4 \mathrm{~K} 2 \mathrm{C}$ ) and covalently attach the photoswitch using the flexible sidechain approach [36] implemented in the Autodock version 4.2 program [37]. We connect one $\mathrm{C} \alpha$ of the maleimide ring to the sulfur atom of Cys34 and allow all rotatable bonds of both cysteine and the attached photoswitch to vary in order to minimize the empirical binding energy of the switch to the protein, while treating the rest of protein as a rigid object. Atomic interactions are modeled using the default parameters of AutoDock 4.2. Docking simulations are performed using the Lamarckian genetic algorithm 
(LGA). We extend the default search parameters for efficient covalent docking of both modified Cys34 residues which have nine rotatable single bonds. The number of individuals in the population is increased to 200, the maximum number of energy evaluations carried out during each LGA run is set to $10^{7}$, and 200 poses are requested in simulation. The resulting poses are clustered with AutoDock with 0.2 nm RMSD tolerance.

The insertion of MO ligand into the IB pocket is also conducted with AutoDock program. We employ the default parameters for the LGA runs, except that we ask for the generation of 50 poses. Equidistant MD snapshots of the IB subdomain taken every $100 \mathrm{ps}$ are used as targets for docking one MO ligand to produce the HSA:1MO complexes. The same procedure is also applied to obtain the HSA:2MO from the MD simulations of the HSA:1MO complexes. We collect the poses that correspond to the most stable configuration of ligand(s) in the IB pocket from each docking simulation, and cluster the compiled frames using the clustering plugin of VMD software [38] with $0.2 \mathrm{~nm}$ RMSD tolerance after aligning the backbone of the IB subdomain.

\subsubsection{Molecular dynamics simulation}

Atomistic MD simulations are performed with the GROMACS version 4.5 package [39], the Amber99SB*-ILDN force field [40,41] for the protein, and generalized amber force field (GAFF) [42] for methyl orange ligand. The construction of force fields of photoswitches and methyl orange ligand is described in the Appendix. We add the missing non-hydrogen atoms in the X-ray structure of HSA using the WHAT IF program [43] and protonate the amino acid residues compatibly to a $\mathrm{pH}$ of 7.5 using the PDB2PQR server [44]. We then solvate the monomeric form of HSA in a 0.15 $\mathrm{M} \mathrm{NaCl}$ water solution in a $12 \times 12 \times 12 \mathrm{~nm}^{3}$ periodic simulation box. The bonded parameters of the water molecules are constrained with the SETTLE algorithm [45]. The electrostatic and van der Waals interactions are evaluated by employing the particle mesh Ewald (PME) method [46] with a cutoff of $1 \mathrm{~nm}$. The structure of the system is then optimized in a steepest-decent energy minimization to avoid clashes among atoms before running any simulations.

In preparation for each MD run of either HSA, HSA-SP, or HSA-MC, the system is first thermally equilibrated to $300 \mathrm{~K}$ in an NVT ensemble for $0.5 \mathrm{~ns}$ with a velocityrescaling thermostat [47] with a coupling constant of $0.1 \mathrm{ps}$. The simulation is then continued in an NPT ensemble for $0.5 \mathrm{~ns}$ to reach a stable pressure of $1 \mathrm{~atm}$ using a Parrinello-Rahman barostat [48] with a coupling constant of $2 \mathrm{ps}$, while maintaining the temperature at $300 \mathrm{~K}$. The LINCS constraint algorithm [49] is applied to all bonds in the protein so that we can use a larger timestep of $2 \mathrm{fs}$. In the temperature and pressure equilibration runs, the positions of non-hydrogen atoms in the protein are restrained with a force constant of $1000 \mathrm{~kJ} \mathrm{~mol}^{-1} \mathrm{~nm}^{-2}$.

Each production run of the HSA-photoswitch configurations consists of three consecutive MD simulations in an NPT ensemble for a total of 170 ns at least. First, we run a $10 \mathrm{~ns}$ MD simulation where we restrain the position of the backbone and the hydrogen-bond distance(s) between the photoswitch and protein. Secondly, we remove the restrain on the backbone in a $40 \mathrm{~ns}$ MD simulation to reach a stable 
conformation that still held the photoswitch-protein hydrogen bond(s). Then, we proceed with a $120 \mathrm{~ns}$ (170 ns for the protein:2MO complexes) simulation without any restraints. For the native HSA simulations, the second step is performed without any restraints. If MO is presence in the IB pocket, the position of its heavy atoms will be restrained only in the first step of simulation. The analysis of MD trajectories is carried out over the last $100 \mathrm{~ns}$ (150 ns for the protein:2MO complexes) of the simulations using equidistant MD snapshots taken every 100 ps.

The global rigidity/flexibility of the protein in an MD simulation is investigated by computing the fluctuations in the distance of the amino acid residues as

$$
f_{i j}=\sqrt{d_{i j}-\bar{d}_{i j}}
$$

where $d_{i j}$ is the distance between the $\mathrm{C} \alpha$ atoms of residues $i$ and $j$, and $\bar{d}_{i j}$ is its average. Residues that lie on the coil structure, including the modified Cys34, are omitted in the calculation as they naturally fluctuate in an uncorrelated way. The square of this quantity is also known in the literature as the coordination propensity [50]. The distance-fluctuation analysis is performed using the CONAN code (https://hits-mbm.github.io/conan). We compute the differential distance-fluctuation matrices between MD simulations to estimate the change in rigidity/flexibility of various regions of the protein and its dependence on the presence of different interactions within the protein.

\subsection{Results and discussions}

\subsubsection{Nature of interaction between HSA and photoswitch}

We perform docking and atomistic MD simulations to elucidate how the photoswitch interacts with the IB pocket. Our docking simulations reveal that the nitro functional group of the photoswitch attached to HSA (PDB entry: 4K2C) can form a hydrogen bond with the Arg144 or the Lys137 residues, which lie on the B2 helix of the IB pocket as shown in Figure 5.3. Further inspection of the amino acids decorating the triangle gate formed by the coil and the B1 and B2 helices corroborates that these two residues are the only candidates for the formation of a hydrogen bond with the photoswitch.

To probe the stability of the noncovalent interactions between HSA and the photoswitch, we start from configurations where the photoswitch is hydrogen-bonded to either Arg144 or Lys137, and perform MD simulations consisting of three steps for a total of at least $170 \mathrm{~ns}$ :

1. In the first $10 \mathrm{~ns}$, we restrain the backbone as well as the photoswitch-IB pocket hydrogen-bond distance(s).

2. In the next $40 \mathrm{~ns}$, we remove the backbone restraint.

3. For the remaining $120 \mathrm{~ns}$, we lift all constraints. 
a)

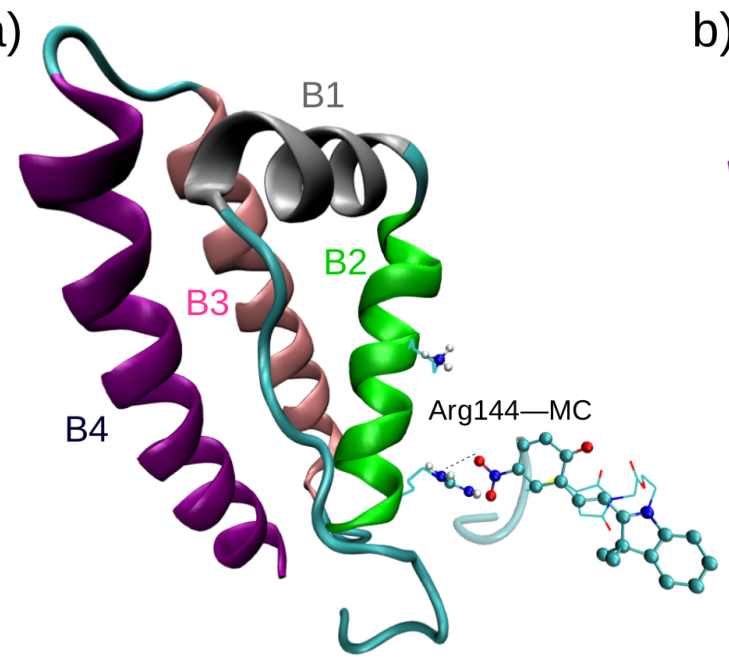

b)

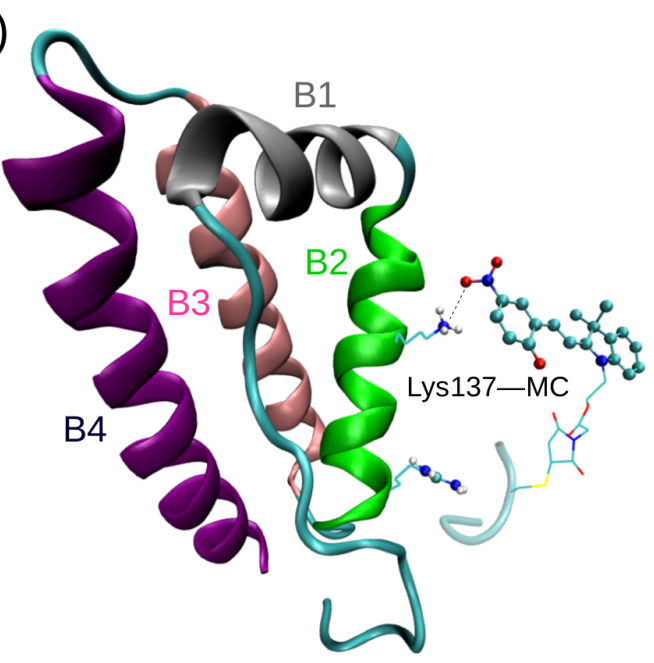

Figure 5.3: Noncovalent interaction between the photoswitch (depicted only for MC) and the (a) Arg144 and (b) Lys137 sidechains of the IB pocket. The pocket consists of a long coil structure and four $\alpha$-helices (B1-B4) connected by three short loops.

Starting from different initial configurations of SP and MC hydrogen-bonded to Arg144, we observe that the bond is preserved for as long as $100 \mathrm{~ns}$ in some MD simulations as shown in Figure 5.4a,b. Therefore, even though the reach of SP is shorter by approximately $0.1 \mathrm{~nm}$ than that of $\mathrm{MC}$ (measured by the distance between $\mathrm{N}_{\text {indole }}$ and $\mathrm{N}_{\text {nitro }}$ ), both switches can form a stable hydrogen bond with this residue. The hydrogen bond of either switch to Lys137 is instead generally weaker and breaks after a few ns of lifting its constraint in the MD simulations as illustrated in Figure 5.4c,d. The photoswitch ring then either moves into bulk solvent, interacts with subdomain IA, or is hydrogen-bonded to the closer Arg144. Two factors contribute to the instability of the switch-Lys137 hydrogen bond. Being surrounded by bulk solvent, the Lys137 sidechain is more flexible than Arg144, which is instead more immersed in the protein environment. Furthermore, Lys137 is located farther by two turns in the $\alpha$-helix structure from the modified Cys34 residue than Arg144.

As the phenoxide oxygen $\left(\mathrm{O}_{\mathrm{ph}}\right)$ of $\mathrm{MC}$ can act as hydrogen-bond acceptor, we also design two possible configurations where MC strongly perturbs the IB pocket and is held inside by hydrogen bonds, as shown in Figure 5.5. First, we force the nitro group inside the IB pocket to hydrogen-bond with Tyr161, and simultaneously form the Lys137- $\mathrm{MC}\left(\mathrm{O}_{\mathrm{ph}}\right)$-Tyr140 hydrogen bond network. The other initial coordinate was constructed by moving the long coil away from the $\alpha$-helices and bridging the Arg114 and Arg144/Arg145 sidechains with the nitrophenoxide group. In both cases, these bonds quickly deteriorate in the MD simulations and the nitrophenoxide group exits the pocket to interact with bulk solvent. We can therefore exclude a direct interference of the photoswitch with the interior of the IB pocket. Based on these findings, we start all MD simulations in the presence of MO with the photoswitch hydrogenbonded to either Arg144 or Lys137. The latter configuration predominantly results again in trajectories where the switch does not interact with the pocket. 

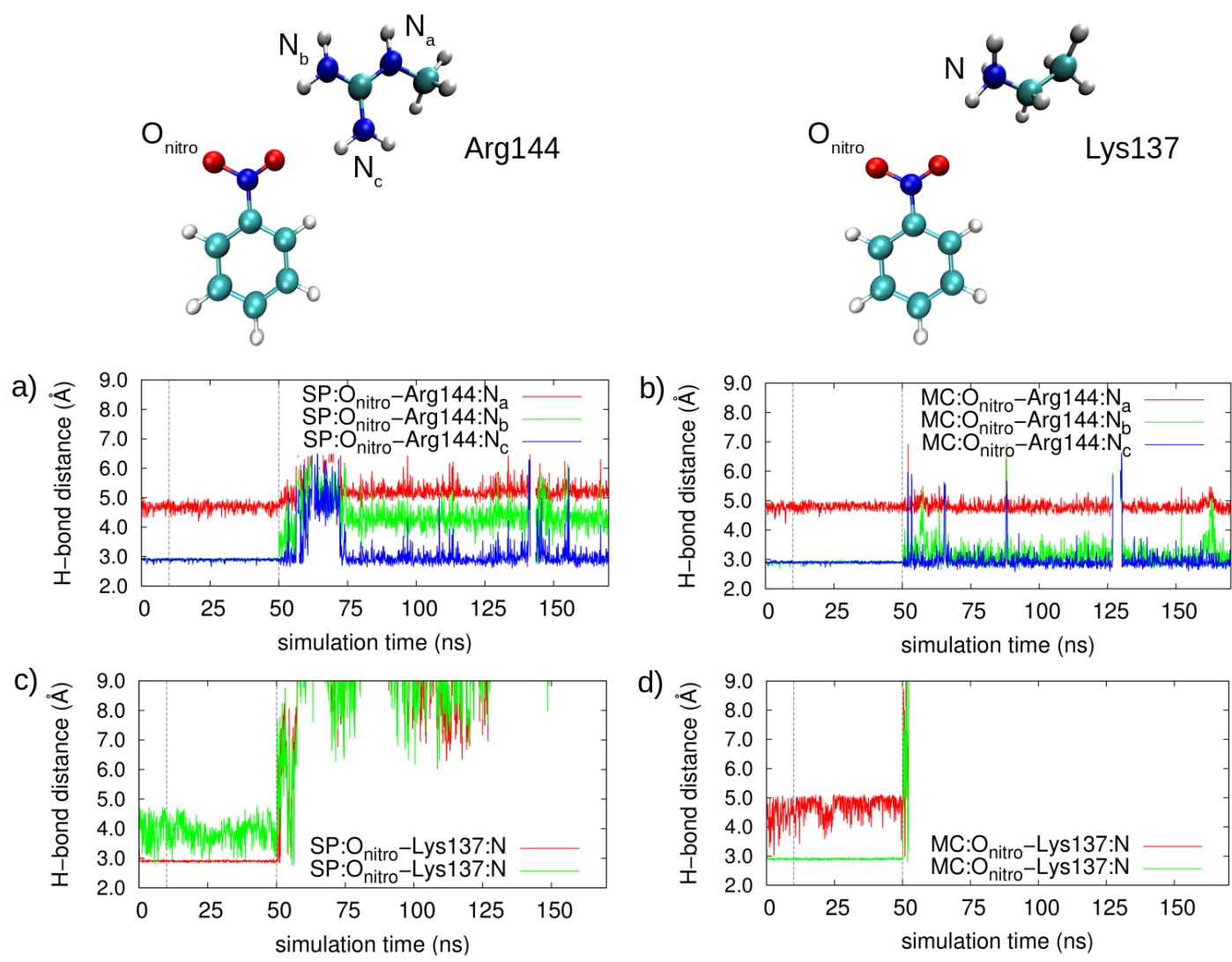

Figure 5.4: Hydrogen-bond distances between the photoswitch and the Arg144 and Lys 137 residues of the IB pocket obtained in four different MD simulations in the absence of MO ligand(s). There are no restraints for $t>50$ ns. For clarity, we always plot the distance between the closest oxygen atom of the nitro group $\left(\mathrm{O}_{\text {nitro }}\right)$ and the three nitrogen atoms of Arg144. a) SP can form a stable hydrogen bond with Arg144. b) MC is hydrogen bonded to Arg144 and the number of its hydrogen bonds is in average higher than that in the HSA-SP system, as indicated by the green line being lower in HSA-MC. Hence, the MC-Arg144 hydrogen bond is stronger than the SP-Arg144 one. The hydrogen bond between the switch and Lys137 is instead not observed (panels $\mathrm{c}$ and $\mathrm{d}$ ). 
a)

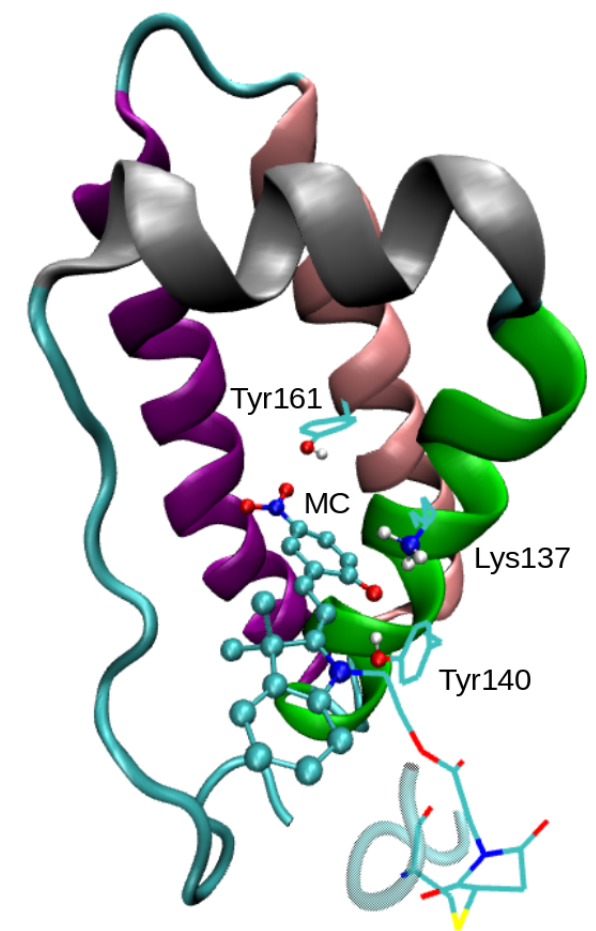

c)

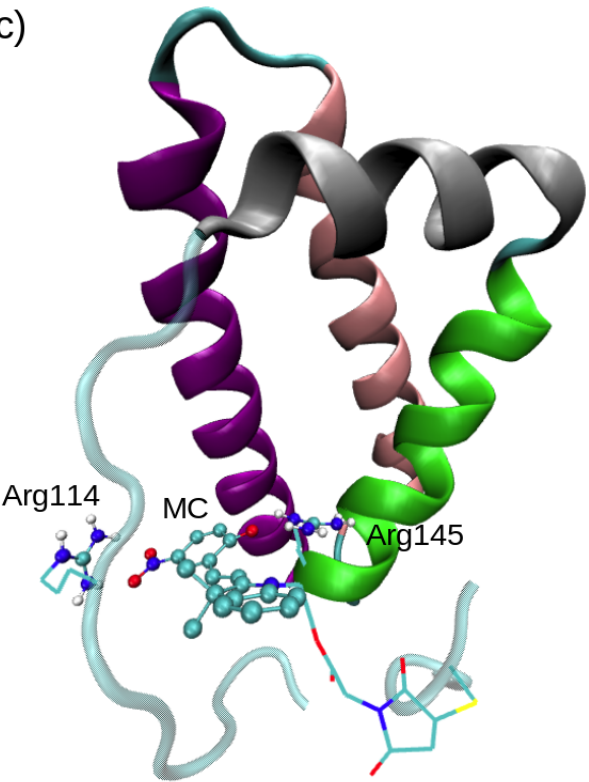

b)
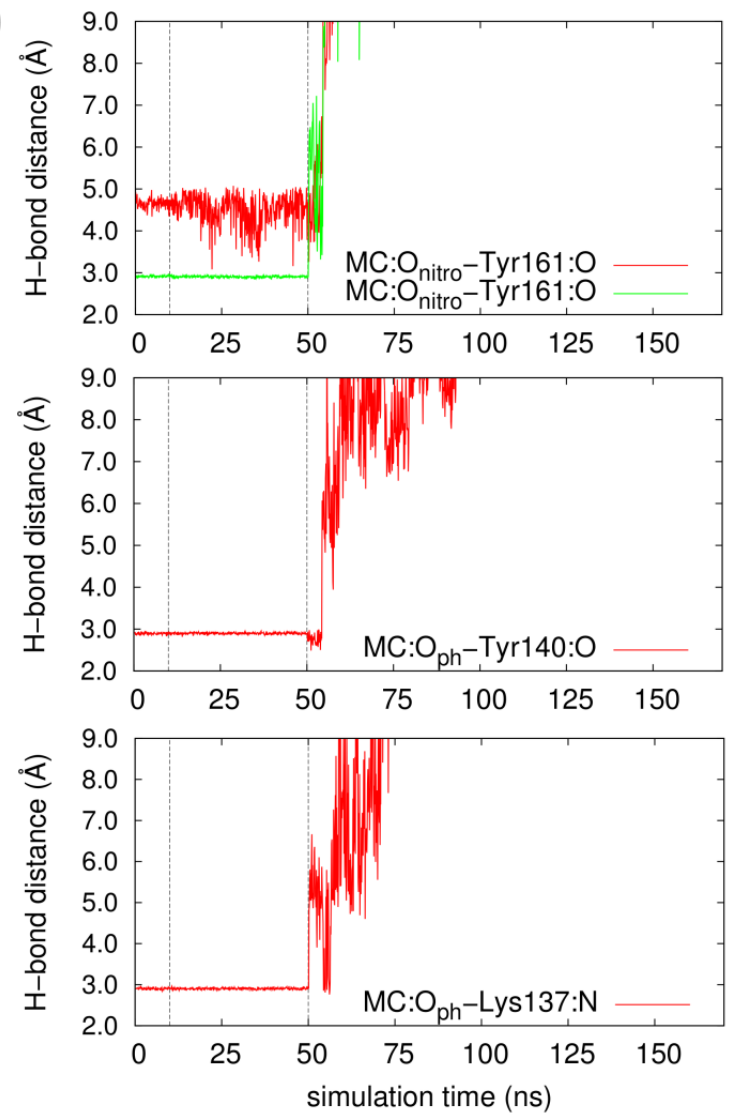

d)
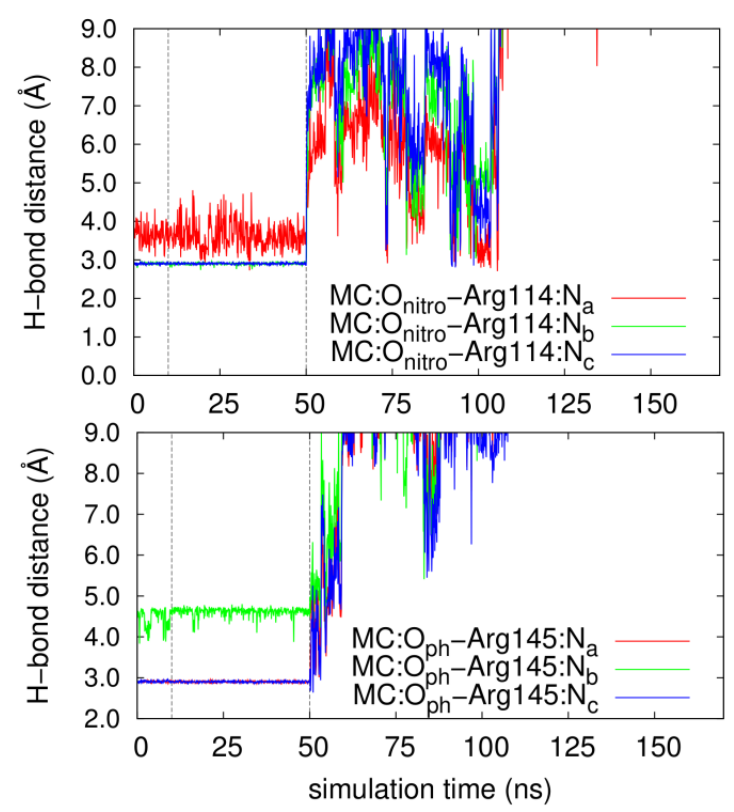

Figure 5.5: Initial configurations of MC pushed towards the interior of the IB pocket (panels a and c) and the time evolution of the distance of the hydrogen bonds that initially maintain the switch inside the pocket (panels b and d). For clarity, we always plot the distance between the closest $\mathrm{O}_{\text {nitro }}$ atom of $\mathrm{MC}$ and the three nitrogen atoms of Arg114. Both initial configurations of MC are found to be unstable in our MD simulations. 


\subsubsection{Mechanistic insight on ligand release}

As in the case of native HSA [51], the IB pocket of our albumin hybrid can also accommodate up to two MOs as indicated by the CD spectra [35]. To generate initial configurations for MD simulations, we dock the MO ligand into multiple host coordinates extracted from the MD simulations of the native and hybrid proteins. The many resulting structures can be grouped in three sets of configurations (A, B, and C) depicted in Figure 5.6. We then dock a second MO ligand in the snapshots extracted from the MD simulations of these HSA:1MO systems. The resulting configurations are combinations of the positions we have identified for a single $\mathrm{MO}$ as also shown in Figure 5.6. For instance, two MOs in position A yield the parallel AA configuration while positions $\mathrm{A}$ and $\mathrm{B}$ give the antiparallel $\mathrm{AB}$ arrangement. We also find the recurrence of the $\mathrm{AC}$ and $\mathrm{CC}$ configurations, while $\mathrm{BB}$ and $\mathrm{BC}$ are not observed, as they are likely characterized by higher binding energies.

Starting from these docking coordinates, we rarely observe direct ligand release in our extensive MD simulations of the native and hybrid systems: the protein binds and dissociates the ligand dynamically in equilibrium, a phenomenon which in average occurs over longer time-scales than computationally accessible. In the 24 simulations (100 ns each) with one MO and 30 simulations (150 ns each) with two MOs, we observe two cases of ligand release, namely, in native HSA with 1) one MO in position A, which exits the pocket between the coil and the B4 helix, and 2) two ligands in the $\mathrm{AC}$ configuration where the $\mathrm{MO}$ at position $\mathrm{A}$ exits the triangle gate. The two events are illustrated in Figures 5.7 and 5.8, respectively. Importantly, in both cases, the release of the MO happens in conjunction with the permanent loss of its hydrogen bond with Lys137, which maintains the ligand at position A. Of the possible MO configurations, the A position is in fact the least stable, having only one hydrogen bond with the binding pocket. 


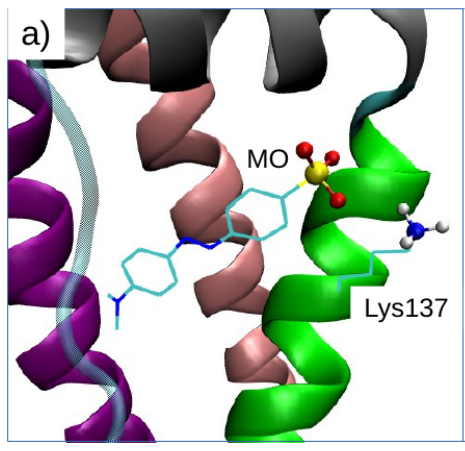

Position A

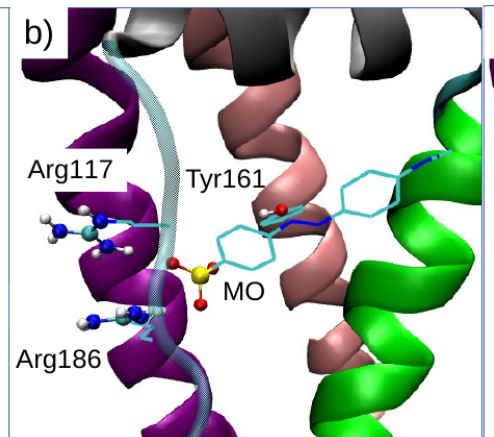

Position B

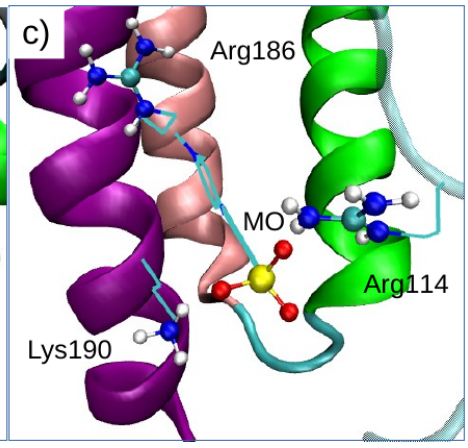

Position C

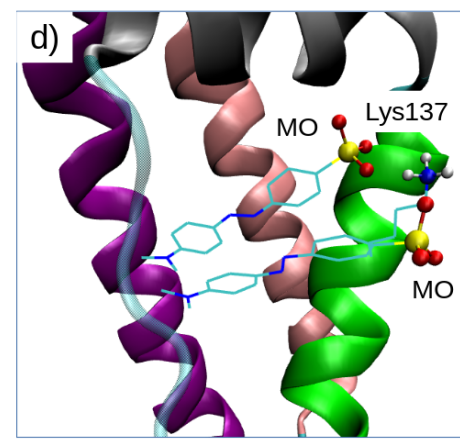

Position AA

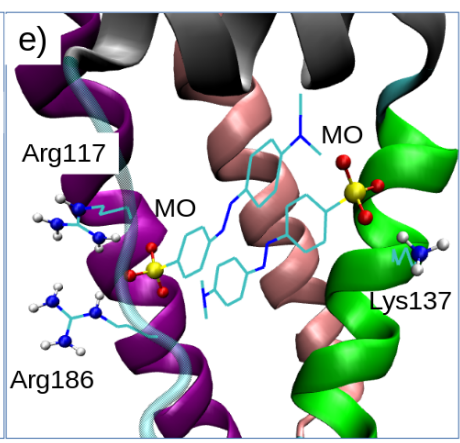

Position $\mathrm{AB}$

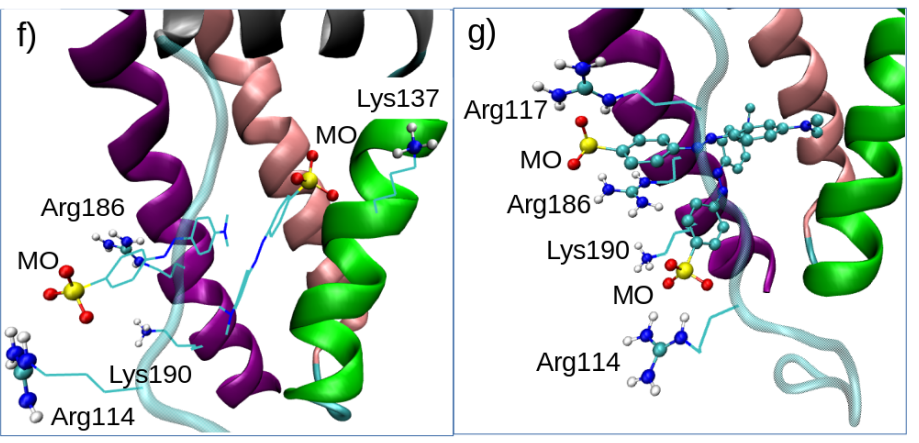

Position AC

Position CC

Figure 5.6: MO configurations in the IB pocket. One ligand: a) MO is kept by a hydrogen bond between its sulfonate group and Lys137; b) the sulfonate group of MO forms three hydrogen bonds with $\operatorname{Arg} 117, \operatorname{Arg} 186$, and Tyr138 of different secondary structures; c) the sulfonate group interacts with Arg114 and Arg117 (not shown) on the coil, and Arg186 and Lys190 on the B4 helix. Two ligands: d) parallel (AA), e) antiparallel (AB), and f) cross (AC) configurations where Lys137 stabilizes the A ligand. g) the $\mathrm{CC}$ configuration of two MO ligands that can be stabilized by four sidechains. 
a)

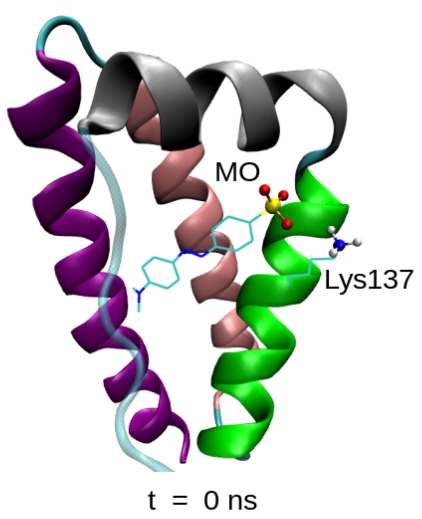

c)

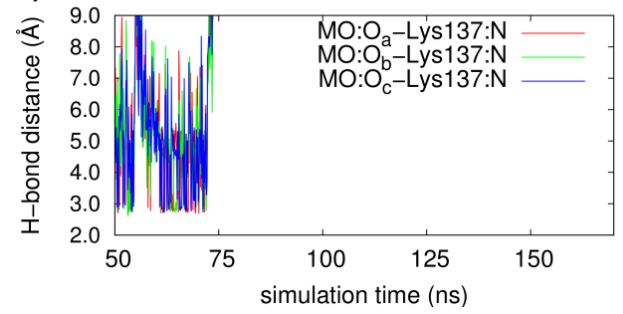

b)

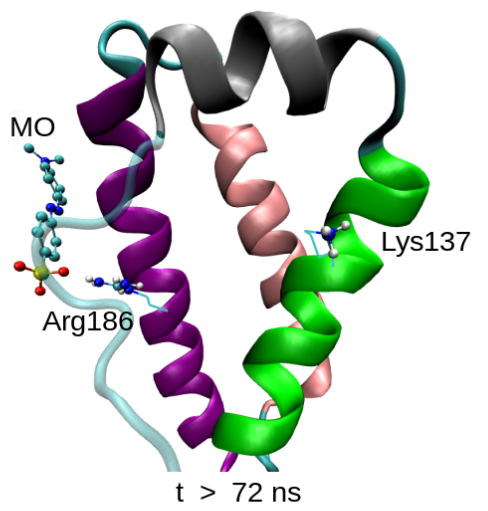

d)

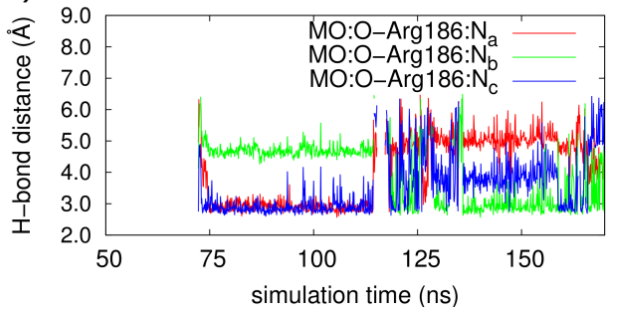

Figure 5.7: Release of MO in the native HSA:1MO complex. a) and b) are two representative snapshots from an MD simulation before and after the release, respectively. c) The MO-Lys137 hydrogen bond is maintained until $72 \mathrm{~ns}$. d) The release then occurs and is followed by the formation of the MO-Arg 186 hydrogen bond in the exterior of the IB pocket. For clarity, we plot the distance between the closest oxygen atom of the sulfonate group of MO and the three nitrogen atoms of Arg186. The MO-Arg186 hydrogen bond will ultimately be lost when MO is dissolved in water.

a)

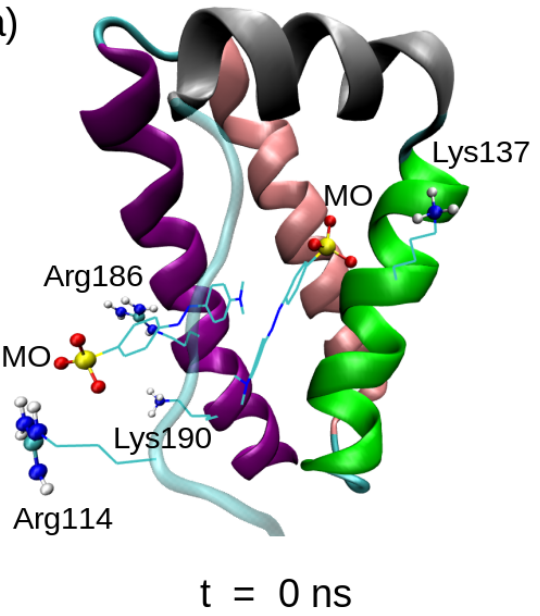

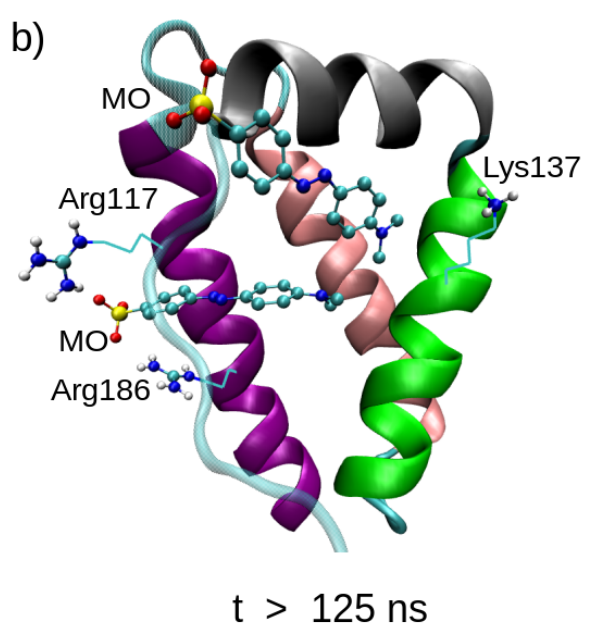

Figure 5.8: Release of one MO molecule in the native HSA:2MO complex in the $\mathrm{AB}$ configuration. a) and b) are two representative snapshots from an MD simulation before and after the release, respectively. The MO-Lys137 bond is lost when the ligand is released and the sulfonate group of MO does not interact with the protein sidechains. 
Given the role of the MO-Lys137 hydrogen bond in stabilizing the ligand at position $\mathrm{A}$, we probe its relative occurrence in the native and hybrid systems. We focus the analysis on the $2 \mathrm{MO}$ complexes (more abundant than the $1 \mathrm{MO}$ ones under the experimental conditions of protein:ligand ratio $=1: 1.3$ ) in the $\mathrm{AB}$ arrangement. Among the three configurations with a MO-Lys137 bond, AB is in fact the most stable and, consequently, the most abundant thermodynamically: the two MOs can form in total three hydrogen bonds with the IB pocket, are $\pi$-stacked, and minimize the repulsion between the negatively-charged sulfonate groups (see Figure 5.6e). The occurrence of the MO-Lys137 hydrogen bond in our MD simulations with two AB MOs is analysed using different time intervals in Table 5.1.

We first note that the occurrence of the MO-Lys 137 hydrogen bond is roughly the same, regardless of the time interval used to compute the average, with the only exception of the HSA-MC complex without MC-Arg144 where larger variations are observed. Then, if we focus on the percentage for the total 70-220 time interval, we find that the occurrence is greater than $90 \%$ in native HSA but is reduced to a maximum of $63 \%$ and $17 \%$ in the presence of SP and MC, respectively. In the HSASP simulations, the presence of a hydrogen bond between SP and Arg144 seems to help preserving the MO-Lys 137 bond whose occurrence increases from $1 \%$ to $63 \%$ when the SP-Arg 144 bond is formed. In the HSA-MC complex, this additional interaction between the IA and IB subdomains via Arg144 has the opposite effect leading to a decrease of the occurrence of the MO-Lys137 bond from a maximum of $17 \%$ to $3 \%$. Overall, these findings indicate that switching spiropyran destabilizes the MO-Lys137 bond and, consequently, facilitates ligand release.

Table 5.1: Occurrence percentage of the MO-Lys137 hydrogen bond in different stretches of the MD trajectories of native and engineered HSA with two MO ligands in the $\mathrm{AB}$ pose. The last line is the average over the complete trajectory (70-220 ns) for each system.

\begin{tabular}{rccccc}
\hline $\begin{array}{r}\text { Time interval } \\
(\mathrm{ns})\end{array}$ & HSA & $\begin{array}{c}\text { HSA-SP } \\
\text { with } \\
\text { SP-Arg144 }\end{array}$ & $\begin{array}{c}\text { HSA-SP } \\
\text { without } \\
\text { SP-Arg144 }\end{array}$ & $\begin{array}{c}\text { HSA-MC } \\
\text { with } \\
\text { MC-Arg144 }\end{array}$ & $\begin{array}{c}\text { HSA-MC } \\
\text { without } \\
\text { MC-Arg144 }\end{array}$ \\
\hline $70-120$ & 96.4 & 68.8 & 1.8 & 2.2 & 9.4 \\
$120-170$ & 93.8 & 51.4 & 0.2 & 3.4 & 40.6 \\
$170-220$ & 88.8 & 69.6 & 0.2 & 4.4 & 1.4 \\
$70-170$ & 95.1 & 60.2 & 1.0 & 2.8 & 25.0 \\
$120-220$ & 91.3 & 60.5 & 0.2 & 3.9 & 21.0 \\
\hline $70-220$ & 93.0 & 63.3 & 0.7 & 3.3 & 17.0 \\
\hline
\end{tabular}

Next, we investigate how the photoswitch affects the coupling and the internal flexibility of the binding IB pocket and the IA subdomain (where the switch is attached) by computing the fluctuations of the $\mathrm{C} \alpha-\mathrm{C} \alpha$ distances between different amino acid residues in our MD trajectories. High fluctuations within IB indicate a greater propensity for ligand dissociation and high cross-fluctuations between IA and IB signal a reduced coordination in their relative motion, which can in turn enhance 


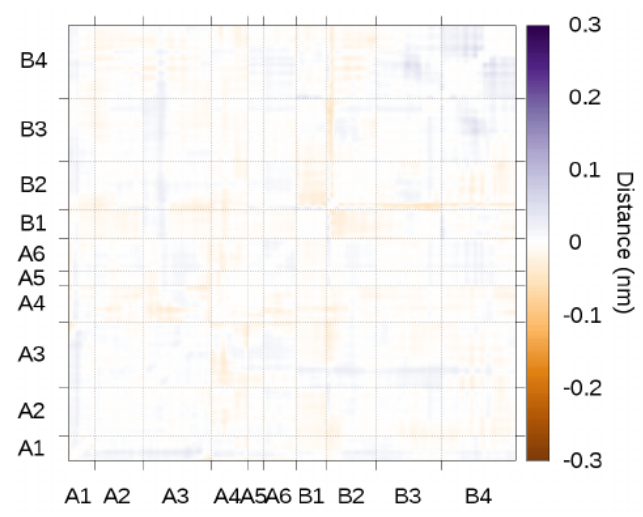

a) HSA-SP vs HSA

(SP is $\mathrm{H}$-bonded to Arg144)

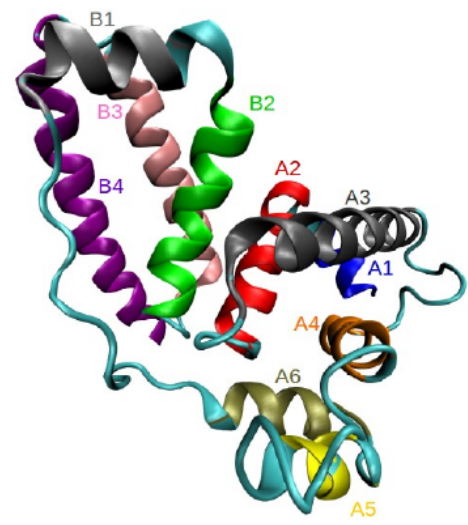

Domain I of HSA

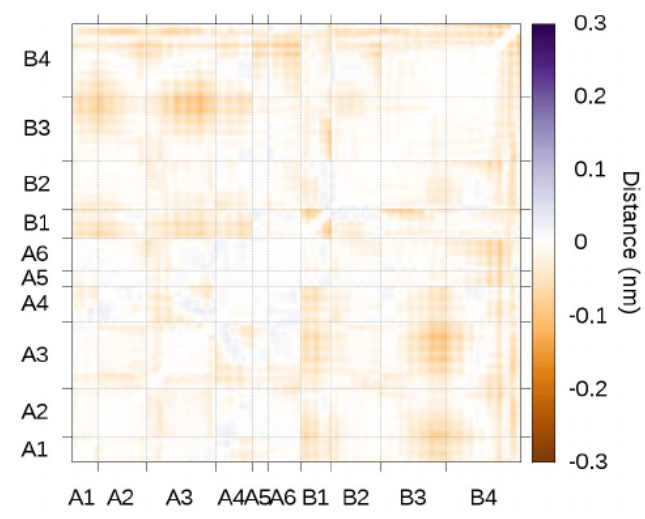

b) HSA-SP vS HSA-MC (H-bonded to Arg144)

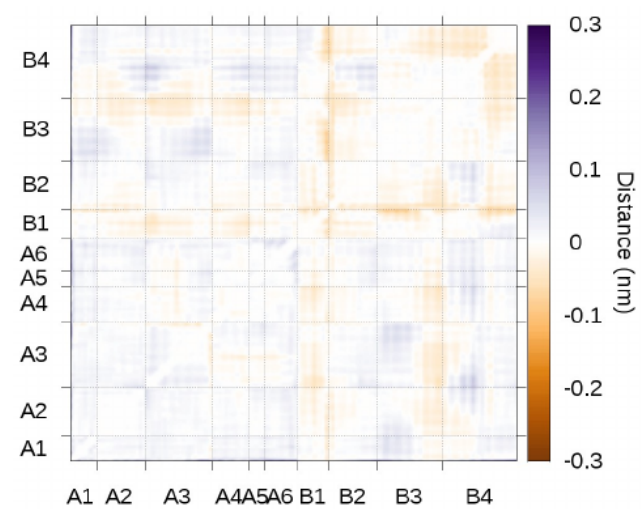

c) HSA-SP vs HSA-MC (no H-bond to Arg144)

Figure 5.9: Comparison of distance fluctuations of domain I of native and engineered HSA complexed with two MO ligands in the AB configuration. a) Highly similar fluctuations of HSA and HSA-SP in the presence of a hydrogen bond to Arg 144 result in extended white (zero) regions, with the exception of the B1-B4 upper-right corner (IB pocket) which becomes slightly more flexible (blue). b) and c) The IB pocket is more flexible upon photoswitching (major orange area in the B1-B4 corner). In b), the enhancement of the cross-fluctuations upon photoswitching in the presence of a hydrogen bond to Arg144 is indicated by the orange area in the (A1-A4, B1-B4) off-diagonal corners. Inset (bottom left): Color-coded structure of domain I.

the overall mobility of the IB pocket and further ease ligand release. Let us start by investigating the effect of attaching the photoswitch to the fluctuation of domain I in the dark. We find that the distance fluctuations of the HSA-SP complex, where SP is predominantly hydrogen-bonded to Arg144, are highly similar to those of the native protein as shown in Figure 5.9a. Therefore, in the presence of SP hydrogen bonded to Arg144, the IB pocket largely maintains its native characteristic dynamical behavior. This is in agreement with the above finding of the occurrence of the MO-Lys137 hydrogen bond remaining as large as $63 \%$ in this configuration. 
To separately investigate the effect of conversion from SP to $\mathrm{MC}$, we focus on the relative fluctuations of the HSA-SP and HSA-MC complexed with two MO ligands in the $\mathrm{AB}$ configuration, which are shown in the presence and the absence of a hydrogen bond between the switch and Arg144 in Figures 5.9b and 5.9c, respectively. In both cases, upon switching, the IB pocket becomes more flexible, with the B1 helix being the most fluctuating $\alpha$-helix in the subdomain. While the presence of MC in this complex appears to be sufficient to increase the flexibility of subdomain IB with respect to the SP case, we find that the additional hydrogen bond to Arg144 enhances the flexibility of all regions and causes the largest changes in the cross-fluctuations of the two subdomains (comparison between panels $\mathrm{b}$ and $\mathrm{c}$ of Figure 5.9). As further illustrated in Figure 5.10, this bond has in fact an opposite effect on the HSA-SP/HSA-MC system, increasing/decreasing the overall rigidity of domain I and, consequently, the stability of the ligand in the pocket as also found before in relation to the occurrence of the MO-Lys137 bond. Furthermore, we analyze the role of the MO ligands in stabilizing the IB pocket in the native and hybrid systems in Figure 5.11. While the presence of the ligands in HSA and HSA-SP reduces the fluctuations of the binding pocket as also observed in other protein-ligand systems, it has the opposite effect in HSA-MC where it enhances the flexibility of the pocket, particularly in the presence of the MC-Arg144 hydrogen bond. All factors therefore concur to facilitate ligand release upon photoswitching.

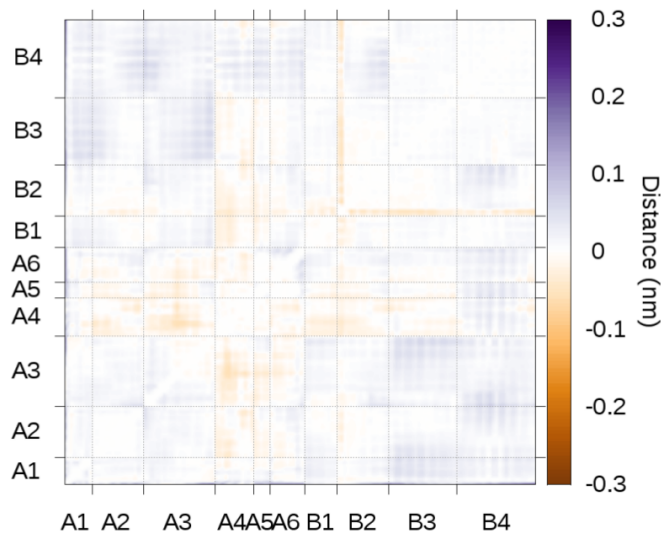

a) HSA-SP in the absence vs presence of $\mathrm{H}$-bond to Arg144

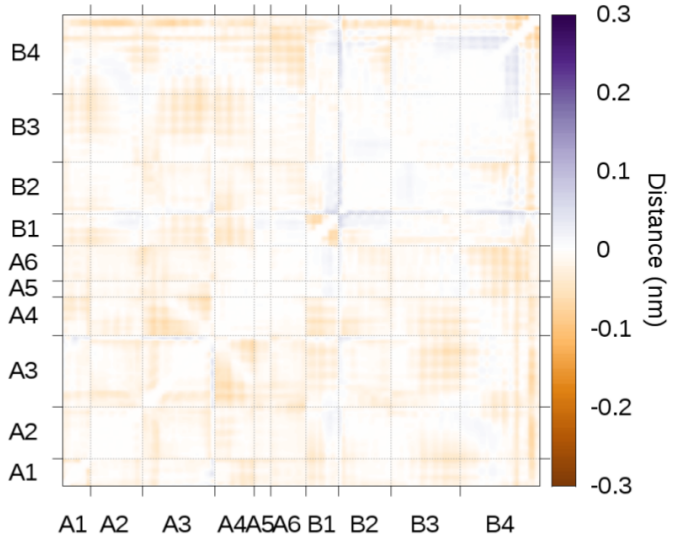

b) HSA-MC in the absence vs presence of $\mathrm{H}$-bond to Arg144

Figure 5.10: Difference of the distance fluctuation matrices of domain I of hybrid HSA in the presence and the absence of a photoswitch-Arg144 hydrogen bond. a) The SP-Arg144 hydrogen bond increases the overall rigidity of domain I, while b) the MC-Arg144 bond enhances the flexibility of this domain. 


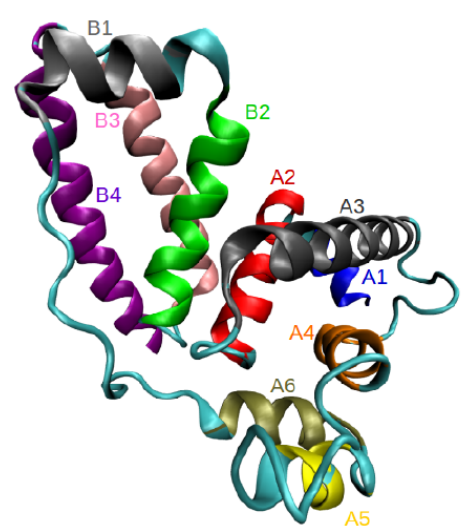

b)

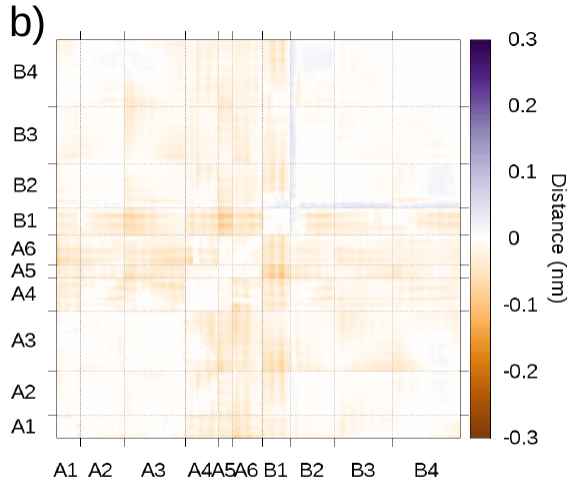

HSA-SP vs HSA-SP:2MO

in the presence of SP-Arg144 H-bond

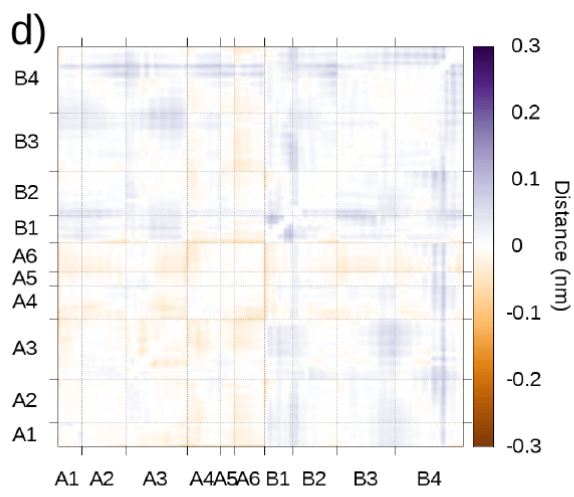

HSA-MC vS HSA-MC: $2 \mathrm{MO}$

in the presence of $\mathrm{MC}$-Arg144 $\mathrm{H}$-bond

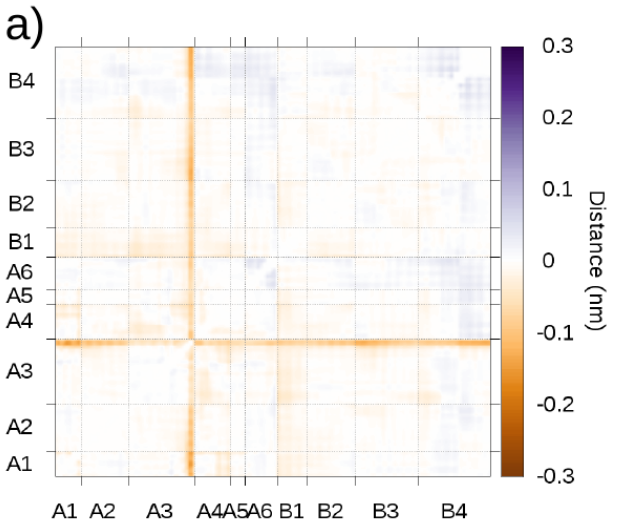

HSA vs HSA:2MO

C)

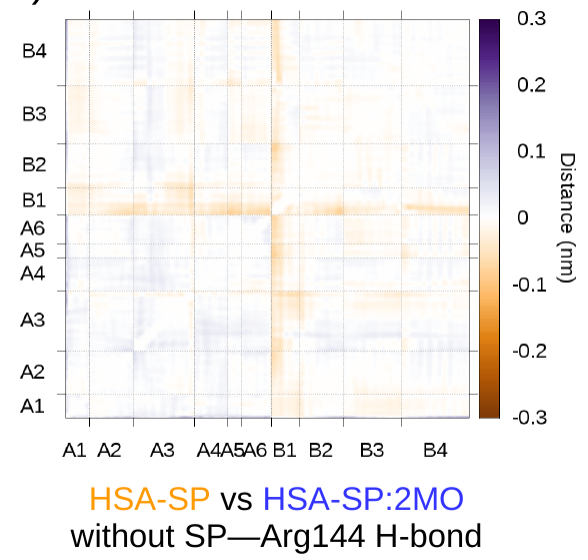

e)

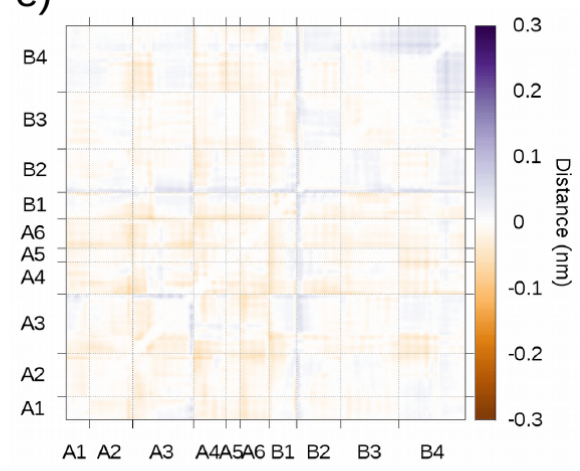

HSA-MC vs HSA-MC:2MO without MC-Arg144 H-bond

Figure 5.11: Comparison of the distance fluctuation matrices between the protein and protein-ligand complexes. Binding ligands decreases the flexibility of the protein binding pocket for a) HSA and b) and c) HSA-SP as demonstrated by a slight blue region in the B1-B4 corner. The presence of the MO ligands in HSA-MC protein, however, gives the opposite effect with the fluctuations of the IB pocket increasing both in d) the presence and e) the absence of the MC-Arg144 hydrogen bond. This finding is an agreement with the experimentally observed release upon SP to MC photoswitching. Inset shows the color-coded secondary structure of domain I. 
Finally, we briefly analyze the parallel AA configuration of the two ligands (see Figure 5.6d) as regards the stability of the MO-Lys 137 hydrogen bond and the internal dynamics of the protein complex. In this arrangement, both negatively-charged sulfonate groups of the MOs point towards bulk solvent and their interaction is mediated by the positively-charged Lys137, which helps overcoming their strong electrostatic repulsion by forming a salt bridge. In our MD simulations, we find however that this salt bridge varies in occurrence and is not well positioned. As detailed in Table 5.2 and Figure 5.12b, even in native HSA, this is responsible for a lower occurrence of the MO-Lys137 hydrogen bond as well as higher fluctuations of the whole domain I. Upon switching from SP to MC, the IB pocket increases its fluctuations (see Figure 5.12b). Moreover, as in the $\mathrm{AB}$ configuration, the motions of the IA and IB subdomains become less coordinated, particularly in the presence of a hydrogen bond between the switch and Arg144. Also for the AA configuration, our findings support a mechanism through which the IB pocket becomes more flexible after photoswitching of the spiropyran, which in turn facilitates the release of the ligands.

Table 5.2: Occurrence of the MO-Lys137 hydrogen bond in two simulations (70-220 ns) of native HSA with two MO ligands in the AA configuration.

\begin{tabular}{lcccc}
\hline & no H-bond & MO1-Lys137 & MO2-Lys137 & MO1-Lys137-MO2 \\
\hline Simulation 1 & 27.5 & 3.3 & 72.2 & 3.0 \\
Simulation 2 & 15.0 & 83.5 & 46.3 & 44.8 \\
\hline
\end{tabular}

a)

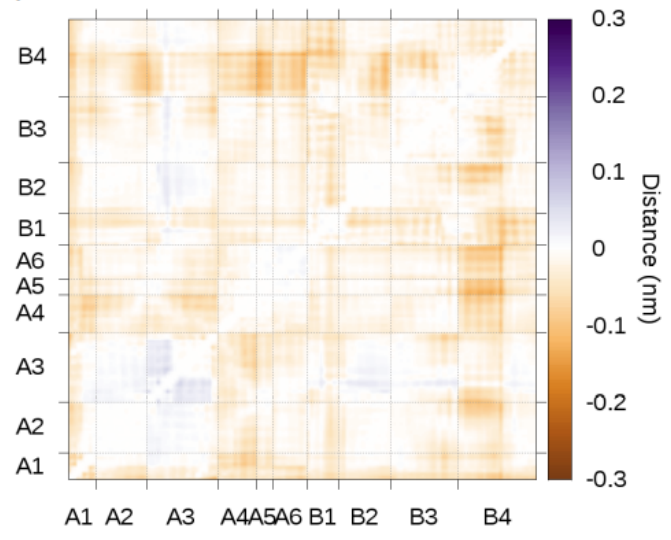

Simulation 1 vs Simulation 2 in native HSA:2MO in AA pose b)

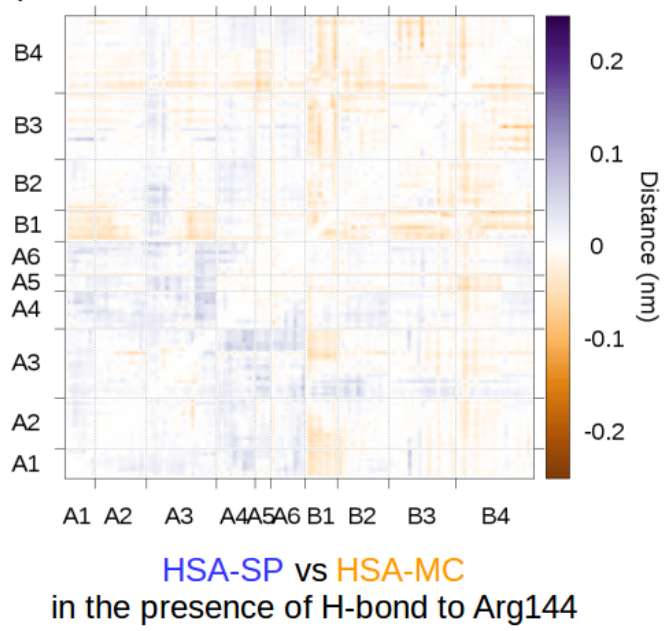

Figure 5.12: Comparison of the distance fluctuation matrices of a) two simulations of native HSA with two MO molecules in the AA configuration and b) HSA-SP and HSA-MC complexed with two MO ligands in the AA configuration. 


\subsection{Conclusions}

A photo-responsive hybrid of the human serum albumin was recently engineered, where irradiation results in ligand release through allosteric communication between subdomains IA and IB, while the overall structure of the protein is preserved. Our molecular dynamics simulations indicate that the covalent attachment of the switch at IA reduces the stability of ligand binding to a key residue of the IB pocket, especially after photo-conversion of the spiropyran switch to merocyanine. Switching correlates with an increased flexibility of the IB pocket and a less coordinated motion of the two subdomains, which further facilitates ligand release. Furthermore, the presence of the ligands acts cooperatively to enhance the fluctuations of the binding pocket.

Allosteric communications between the IB and IA subdomains of albumin is unprecedented. The remote control of a binding pocket by switching at a neighboring site opens new opportunities for pre-programming and photo-controlling allosteric regulation externally, as well as inducing new channels of allosteric interaction in the human serum albumin. We envision that similar engineering strategies could be extended to systems with higher-order structures and further on to supramolecular protein-based hybrids [52]. 


\subsection{Appendix: Force-field construction}

Since the photoswitches are attached covalently to the protein, we construct their force field to be compatible with Amber99SB*-ILDN which is used for the rest of the protein. The force field for the MO ligand is built according to GAFF recipe.

Lennard-Jones parameters for each atom in the photoswitches are determined based on the similarity of the atoms already defined in the force field. Atomic charges of the modified cysteine residue are derived according to the multi-conformational restrained electrostatic potential (RESP) approach [53]. Geometries are optimized at the HF/6-31G* level and the charges are calculated with the same theory using the Gaussian software [54]. When fitting atomic charges of an amino-acid residue (RES), one typically caps it with an acetil group (ACE) at the N-terminus and a methylamine (NME) at the C-terminus, and perform the multi-conformational fit to the resulting ACE-RES-NME tripeptide, while restraining the charges of the capping groups to predefined values. This procedure, however, is difficult to implement in our Cysmaleimide-SP and Cys-maleimide-MC systems since they many conformers due to the presence of at least six rotatable single bonds.

a)

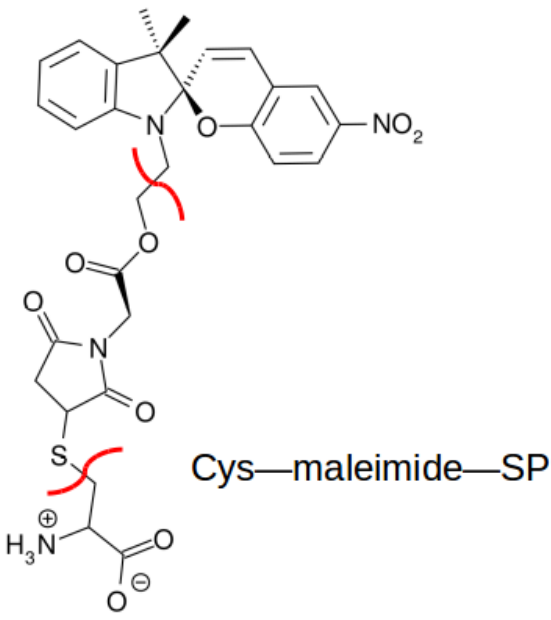

b)

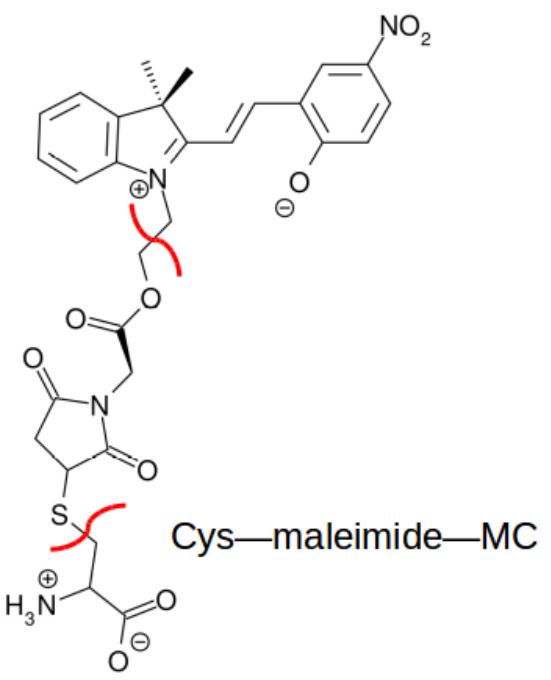

c)

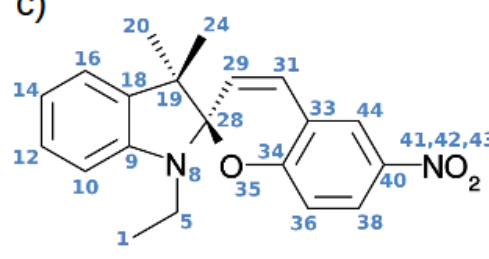

SP d)

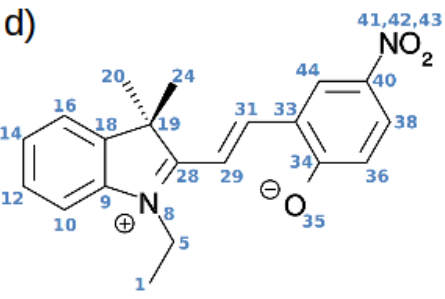

MC e)<smiles>CCOC(=O)CN1C(=O)CC(SC)C1=O</smiles>

Figure 5.13: Partitioning (a) the Cys-maleimide-SP and (b) the Cys-maleimide-MC systems for a simpler RESP charge derivation. Red curly lines indicate the location of the cut yielding four smaller molecules: cysteine, c) SP, d) MC, and e) maleimide anchor. Nonhydrogen atoms are numbered in blue. 
We therefore simplify the task by homolitically partitioning the system into three pieces (Figures 5.13a-b), and cap the dangling bond of each smaller radical compound with a methyl group (Figures $5.13 \mathrm{c}-\mathrm{e}$ ). By doing so, we do not only reduce the computational cost but can also employ the same parameters for the components common to the two systems (i.e. cysteine and maleimide anchor). The multiconformers RESP charges are then fitted for these smaller compounds, except for the cysteine part without the thiol group, whose charges remain unaltered with respect to the original Amber99SB*-ILDN force field. The charges of the three parts without those of the capping methyl groups are added up to obtain the charge of the whole modified cysteine residue. As this procedure does not guarantee a neutral total charge, we proportionally distribute the excess of charge to all atoms weighted by their atomic mass.

The atomic charges for each capped molecule are further averaged over four minimum structures. For SP, these geometries are characterized by the different values in the dihedral angles $\mathrm{N}_{8}-\mathrm{C}_{28}-\mathrm{C}_{29}-\mathrm{C}_{31}$ [55] and $\mathrm{C}_{1}-\mathrm{C}_{5}-\mathrm{N}_{8}-\mathrm{C}_{28}$, where the atoms are numbered as in Figure 5.13. The four lowest isomers of $\mathrm{MC}$ are obtained by a $180^{\circ}$ rotation of two dihedral angles of the bridge, i.e. $\mathrm{N}_{8}-\mathrm{C}_{28}-\mathrm{C}_{29}-\mathrm{C}_{31}$ and $\mathrm{C}_{29}-\mathrm{C}_{31}-\mathrm{C}_{33}-$ $\mathrm{C}_{34}$. Rotating the middle bond of the $\mathrm{MC}$ bridge, $\mathrm{C}_{28}-\mathrm{C}_{29}-\mathrm{C}_{31}-\mathrm{C}_{33}$, yields minima which are higher in energy due to the steric hindrance of the two rings. For the maleimide anchor, the lowest four conformers are found by varying $\mathrm{C}_{11}-\mathrm{N}_{15}-\mathrm{C}_{16}-$ $\mathrm{C}_{19}$ and $\mathrm{N}_{15}-\mathrm{C}_{16}-\mathrm{C}_{19}-\mathrm{O}_{21}$. Modifying the $\mathrm{C}_{16}-\mathrm{C}_{19}-\mathrm{O}_{21}-\mathrm{C}_{22}$ torsional angle is not energetically favorable because of the preferred $\mathrm{Z}$-conformation of the aliphatic ester over the E-conformation by approximately $30 \mathrm{~kJ} / \mathrm{mol}$. This arises from the dipole minimization and the hyperconjugation of the lone-pair electron of the $\alpha$-oxygen with the neighboring $\sigma^{*}$ orbitals of the carbonyl group. The relative energies of the four minima of SP, MC, and the maleimide anchor are given in Table 5.3.

For the bonded force field, the equilibrium geometrical parameters are taken from the optimal B3LYP/6-31G* structures in vacuum except for the cysteine part where we employ the default Amber99 parameters. Most of the force constants are determined based on the similarity with those of bond lengths, bond angles, and torsional angles already existing in the Amber99SB*-ILDN force-field database. Nonstandard constants are either taken from the literature or determined in this work. The nitro-related force constants obtained compatibly with the Amber99 force field for the nitrated aromatic amino acid residues (i.e. tyrosine and tryptophan) in Ref. [56] are here directly applied to our SP and MC photoswitches. The remaining nonstandard force constants, which are parameterized in this work, are those of the torsional angle of the merocyanine bridge.

To generate the proper dihedral angle parameters of the three aliphatic conjugated bonds of $\mathrm{MC}$, we construct a minimal and universal model of MC by eliminating a benzene ring and two methyl groups on the indoline ring, and the nitro group on the phenoxide ring as depicted in Figure 5.14. We consider four models labeled with three letters $\mathrm{XXX}$ with $\mathrm{X}=\mathrm{T}$ for trans and $\mathrm{X}=\mathrm{C}$ for cis, according to the stereoisomer of the three dihedral angles $(\alpha, \beta, \gamma)$ of the bridge and moving from the isopyrrole cation to the phenoxide ring. Here, we aim to reproduce the quantum mechanical (QM) B3LYP/6-311+G(2d,p) relative energies and appropriate barrier heights of cis- 
Table 5.3: Relative electronic energies of the four most stable conformers of SP, MC, and the maleimide anchor in $\mathrm{kJ} / \mathrm{mol}$.

\begin{tabular}{lrrr}
\hline SP & $\operatorname{Dih}\left(\mathrm{N}_{8}-\mathrm{C}_{28}-\mathrm{C}_{29}-\mathrm{C}_{31}\right)$ & $\mathrm{Dih}\left(\mathrm{C}_{1}-\mathrm{C}_{5}-\mathrm{N}_{8}-\mathrm{C}_{28}\right)$ & Energy \\
\hline SP1 & -125.2 & 83.8 & 1.6 \\
SP2 & -115.3 & 123.2 & 10.3 \\
SP3 & -124.3 & -127.5 & 0.0 \\
SP4 & -119.0 & -89.7 & 14.5 \\
\hline MC & $\operatorname{Dih}\left(\mathrm{N}_{8}-\mathrm{C}_{28}-\mathrm{C}_{29}-\mathrm{C}_{31}\right)$ & $\operatorname{Dih}\left(\mathrm{C}_{29}-\mathrm{C}_{31}-\mathrm{C}_{33}-\mathrm{C}_{34}\right)$ & Energy \\
\hline MC1 & 180.0 & 0.0 & 0.0 \\
MC2 & 180.0 & 180.0 & 7.2 \\
MC3 & 0.0 & 0.0 & 10.8 \\
MC4 & 0.0 & 180.0 & 13.8 \\
\hline Mal & $\mathrm{Dih}\left(\mathrm{C}_{11}-\mathrm{N}_{15}-\mathrm{C}_{16}-\mathrm{C}_{19}\right)$ & $\mathrm{Dih}\left(\mathrm{N}_{15}-\mathrm{C}_{16}-\mathrm{C}_{19}-\mathrm{O}_{21}\right)$ & Energy \\
\hline $\mathrm{Mal}_{1}$ & 270.8 & 180.3 & 2.3 \\
$\mathrm{Mal}_{2}$ & 84.8 & 179.2 & 0.0 \\
$\mathrm{Mal}_{3}$ & 284.5 & -13.4 & 4.9 \\
$\mathrm{Mal}_{4}$ & 87.6 & -1.5 & 5.1 \\
\hline
\end{tabular}

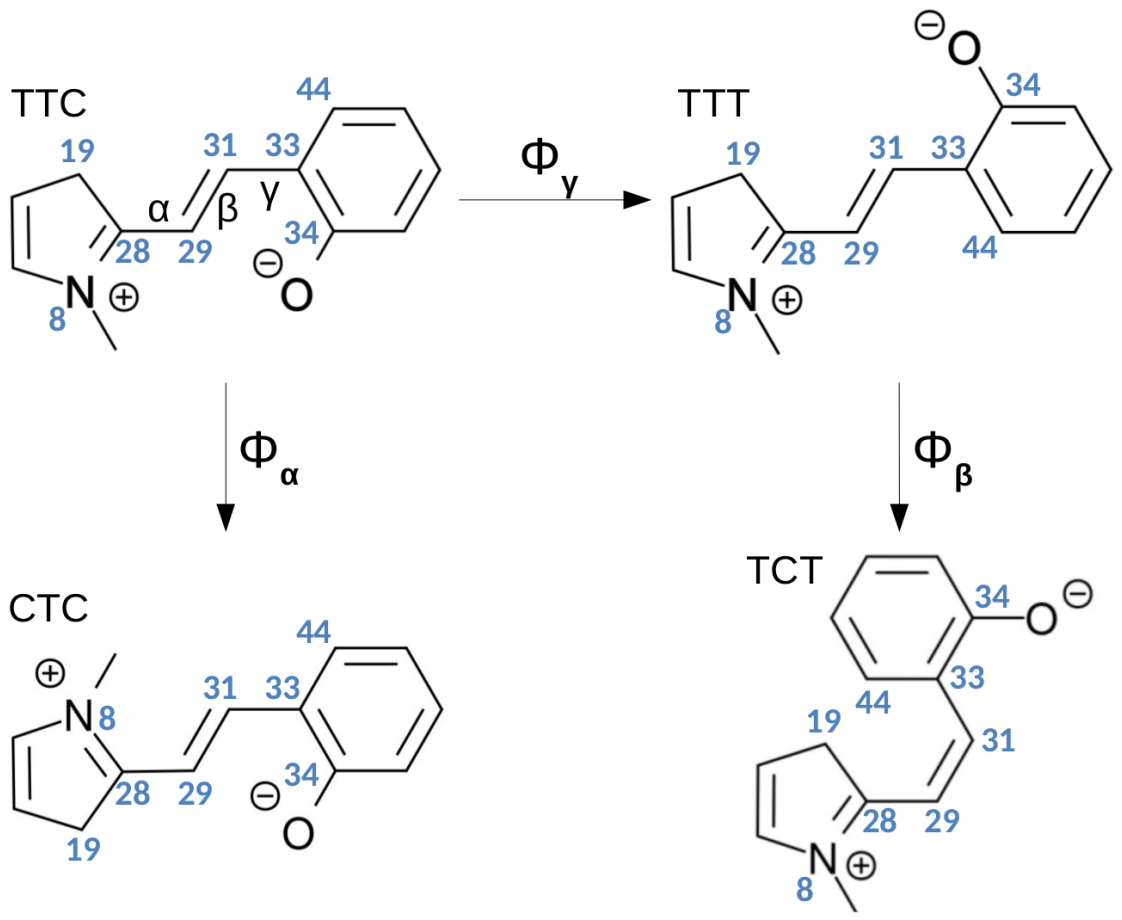

Figure 5.14: Merocyanine minimal and universal models used to parameterize the three torsional angle potentials of the bridge: $V(\alpha), V(\beta)$, and $V(\gamma)$. Atoms are numbered similarly to the original MC. 
trans isomerizations in vacuum of four MC model conformers i.e. TTC, TTT, CTC, and TCT, with molecular mechanics (MM). The relative energies are reproduced by adjusting the $\mathrm{V}_{1}$ torsional term of the force field, while the barriers are adjusted via the $\mathrm{V}_{2}$ term. In doing so, we arrive at the following equations for $V(\alpha), V(\beta)$, and $V(\gamma)$ :

$$
\begin{aligned}
& V(\alpha)=V\left(\Phi_{\mathrm{X}-\mathrm{C}_{28}-\mathrm{C}_{29}-\mathrm{X}}\right)=V_{1, \alpha}\left[1+\cos \left(\Phi_{\mathrm{N}_{8}-\mathrm{C}_{28}-\mathrm{C}_{29}-\mathrm{C}_{31}}\right)\right] \\
& +V_{2, \alpha}\left[1+\cos \left(2 \Phi_{\mathrm{N}_{8}-\mathrm{C}_{28}-\mathrm{C}_{29}-\mathrm{C}_{31}}\right)-\pi\right] \\
& +V_{1, \alpha}\left[1+\cos \left(\Phi_{\mathrm{N}_{8}-\mathrm{C}_{28}-\mathrm{C}_{29}-\mathrm{H}_{30}}\right)-\pi\right] \\
& +V_{2, \alpha}\left[1+\cos \left(2 \Phi_{\mathrm{N}_{8}-\mathrm{C}_{28}-\mathrm{C}_{29}-\mathrm{H}_{30}}\right)-\pi\right] \\
& +V_{1, \alpha}\left[1+\cos \left(\Phi_{\mathrm{C}_{19}-\mathrm{C}_{28}-\mathrm{C}_{29}-\mathrm{C}_{31}}\right)-\pi\right] \\
& +V_{2, \alpha}\left[1+\cos \left(2 \Phi_{\mathrm{C}_{19}-\mathrm{C}_{28}-\mathrm{C}_{29}-\mathrm{C}_{31}}\right)-\pi\right] \\
& +V_{1, \alpha}\left[1+\cos \left(\Phi_{\mathrm{C}_{19}-\mathrm{C}_{28}-\mathrm{C}_{29}-\mathrm{H}_{30}}\right)\right] \\
& +V_{2, \alpha}\left[1+\cos \left(2 \Phi_{\mathrm{C}_{19}-\mathrm{C}_{28}-\mathrm{C}_{29}-\mathrm{H}_{30}}\right)-\pi\right] \\
& V(\beta)=V\left(\Phi_{\mathrm{X}-\mathrm{C}_{29}-\mathrm{C}_{31}-\mathrm{X}}\right)=V_{1, \beta}\left[1+\cos \left(\Phi_{\mathrm{C}_{28}-\mathrm{C}_{29}-\mathrm{C}_{31}-\mathrm{C}_{33}}\right)\right] \\
& +V_{2, \beta}\left[1+\cos \left(2 \Phi_{\mathrm{C}_{28}-\mathrm{C}_{29}-\mathrm{C}_{31}-\mathrm{C}_{33}}\right)-\pi\right] \\
& +V_{1, \beta}\left[1+\cos \left(\Phi_{\mathrm{C}_{28}-\mathrm{C}_{29}-\mathrm{C}_{31}-\mathrm{H}_{32}}\right)-\pi\right] \\
& +V_{2, \beta}\left[1+\cos \left(2 \Phi_{\mathrm{C}_{28}-\mathrm{C}_{29}-\mathrm{C}_{31}-\mathrm{H}_{32}}\right)-\pi\right] \\
& +V_{1, \beta}\left[1+\cos \left(\Phi_{\mathrm{H}_{30}-\mathrm{C}_{29}-\mathrm{C}_{31}-\mathrm{C}_{33}}\right)-\pi\right] \\
& +V_{2, \beta}\left[1+\cos \left(2 \Phi_{\mathrm{H}_{30}-\mathrm{C}_{29}-\mathrm{C}_{31}-\mathrm{C}_{33}}\right)-\pi\right] \\
& +V_{1, \beta}\left[1+\cos \left(\Phi_{\mathrm{H}_{30}-\mathrm{C}_{29}-\mathrm{C}_{31}-\mathrm{H}_{32}}\right)\right] \\
& +V_{2, \beta}\left[1+\cos \left(2 \Phi_{\mathrm{H}_{30}-\mathrm{C}_{29}-\mathrm{C}_{31}-\mathrm{H}_{32}}\right)-\pi\right] \\
& V(\gamma)=V\left(\Phi_{\mathrm{X}-\mathrm{C}_{31}-\mathrm{C}_{33}-\mathrm{X}}\right)=V_{1, \gamma}\left[1+\cos \left(\Phi_{\mathrm{C}_{29}-\mathrm{C}_{31}-\mathrm{C}_{33}-\mathrm{C}_{44}}\right)\right] \\
& +V_{2, \gamma}\left[1+\cos \left(2 \Phi_{\mathrm{C}_{29}-\mathrm{C}_{31}-\mathrm{C}_{33}-\mathrm{C}_{44}}\right)-\pi\right] \\
& +V_{1, \gamma}\left[1+\cos \left(\Phi_{\mathrm{C}_{29}-\mathrm{C}_{31}-\mathrm{C}_{33}-\mathrm{C}_{34}}\right)-\pi\right] \\
& +V_{2, \gamma}\left[1+\cos \left(2 \Phi_{\mathrm{C}_{29}-\mathrm{C}_{31}-\mathrm{C}_{33}-\mathrm{C}_{34}}\right)-\pi\right] \\
& +V_{1, \gamma}\left[1+\cos \left(\Phi_{\mathrm{H}_{32}-\mathrm{C}_{31}-\mathrm{C}_{33}-\mathrm{C}_{44}}\right)-\pi\right] \\
& +V_{2, \gamma}\left[1+\cos \left(2 \Phi_{\mathrm{H}_{32}-\mathrm{C}_{31}-\mathrm{C}_{33}-\mathrm{C}_{44}}\right)-\pi\right] \\
& +V_{1, \gamma}\left[1+\cos \left(\Phi_{\mathrm{H}_{32}-\mathrm{C}_{31}-\mathrm{C}_{33}-\mathrm{C}_{44}}\right)\right] \\
& +V_{2, \gamma}\left[1+\cos \left(2 \Phi_{\mathrm{H}_{32}-\mathrm{C}_{31}-\mathrm{C}_{33}-\mathrm{C}_{44}}\right)-\pi\right] \text {. }
\end{aligned}
$$

For each dihedral angle, we perform a potential energy scan both in the forward and the backward direction, and show the resulting energy profiles in Figure 5.15. We find that the direction of scan does not affect the potential energy curve of $V(\beta)$, while it leads to different energy profiles for $V(\alpha)$ and $V(\gamma)$ in the vicinity of the transition state and, correspondingly, to distinct optimal values for the other dihedral angles. Therefore, the three dihedral angles are strongly coupled and their parameterization requires an iterative procedure as follows.

We start by parameterizing $V(\alpha)$ using the TTC $\rightarrow$ CTC quantum-mechanical energy profile and geometries, while setting $V(\beta)=V(\gamma)=0$. The resulting $V(\alpha)$ and $V(\gamma)=0$ are used to parameterize $V(\beta)$ according to the TTT $\rightarrow$ TCT energy 
curve. Next, $V(\alpha)$ and $V(\beta)$ are employed to determine $V(\gamma)$ using the TTC $\rightarrow$ TTT energy scan. Having completed this first iteration, one realizes that the current $V(\alpha)$ is no longer optimal, since it was parameterized in the absence of the other two dihedral-angle force fields. Therefore, we proceed to the second iteration starting from the parameterization of $V(\alpha)$ with existing $V(\beta)$ and $V(\gamma)$, and carry on the process until $V(\alpha), V(\beta)$, and $V(\gamma)$ reach consistent values. We note that, due to the distinct energy profile in the vicinity of the transition states (see Figure 5.15a,e), we assume that the transition states are located exactly at $90^{\circ}$ and $270^{\circ}$, and average the energy from both directions to estimate the barrier height for the parameterization of the $\mathrm{V}_{2}$ term.

We list the optimized parameters in Table 5.4 and employ them in the constrained geometry optimization at the MM level in order to validate our force field. We find that our iterative procedure satisfactorily yields compatible potential energy profiles at the MM level to those at the QM level as demonstrated in Figure 5.16. In particular, the location of a local minima along the scan of $\beta$ dihedral angle is very well reproduced even though its geometry is not considered during the parameterization. Further improvement in the agreement between the QM and MM potential energy curves with the inclusion of higher order potentials (e.g. $\mathrm{V}_{3}, \mathrm{~V}_{4}$, etc.), is not necessary as the MM curve with two parameters for each dihedral angle already yields a reliable representation of the QM calculations.

Table 5.4: Optimized force-field parameters in $\mathrm{kJ} / \mathrm{mol}$ for the dihedral angle of the MC model bridge.

\begin{tabular}{crr}
\hline Dihedral angle & $\mathrm{V}_{1}$ & $\mathrm{~V}_{2}$ \\
\hline$\alpha$ & 1.3 & 19.4 \\
$\beta$ & -2.1 & 11.6 \\
$\gamma$ & 2.2 & 22.1 \\
\hline
\end{tabular}

Finally, we describe the force-field construction for the MO ligand according to the GAFF recipe. We apply the GAFF parameters of the substituted azobenzene dye [57] to the MO ligand. Missing parameters are filled with standard GAFF parameters except for the torsional angles of the sulfonate group, which we independently parameterize against the $\mathrm{B} 3 \mathrm{LYP} / 6-311+\mathrm{G}(2 \mathrm{~d}, \mathrm{p})$ potential energy curve in vacuum. The existing GAFF torsional potential for the similar sulfone group, $\mathrm{RSO}_{2} \mathrm{R}^{\prime}$, is too rigid for the sulfonate group that can rotate freely at room temperature (the barrier being less than $\mathrm{k}_{\mathrm{B}} \mathrm{T}$ ). Our optimized potential for the dihedral angle of the sulfonate group is

$$
V\left(\Phi_{\mathrm{X}-\mathrm{C}-\mathrm{S}-\mathrm{X}}\right)=0.011\left[1+\cos \left(6 \Phi_{\mathrm{X}-\mathrm{C}-\mathrm{S}-\mathrm{X}}\right)\right] \text {. }
$$



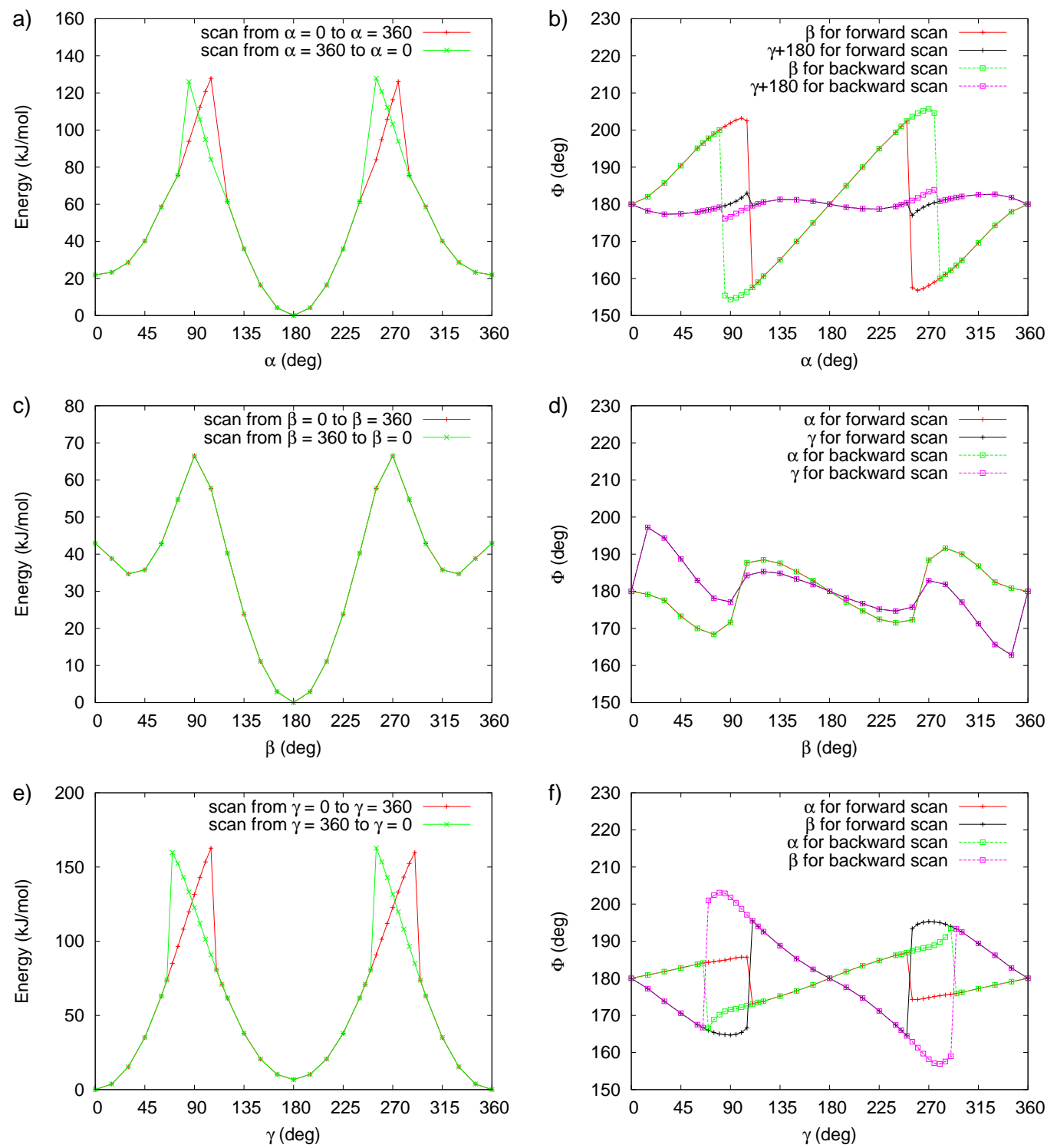

Figure 5.15: a), c), e) Potential energy profiles for the rotation around the $\alpha, \beta$, and $\gamma$ dihedral angles of the universal model of MC at the B3LYP/6-311+G(2d,p) level in vacuum and $b$ ), d), f) corresponding evolution of the other dihedral angles. 

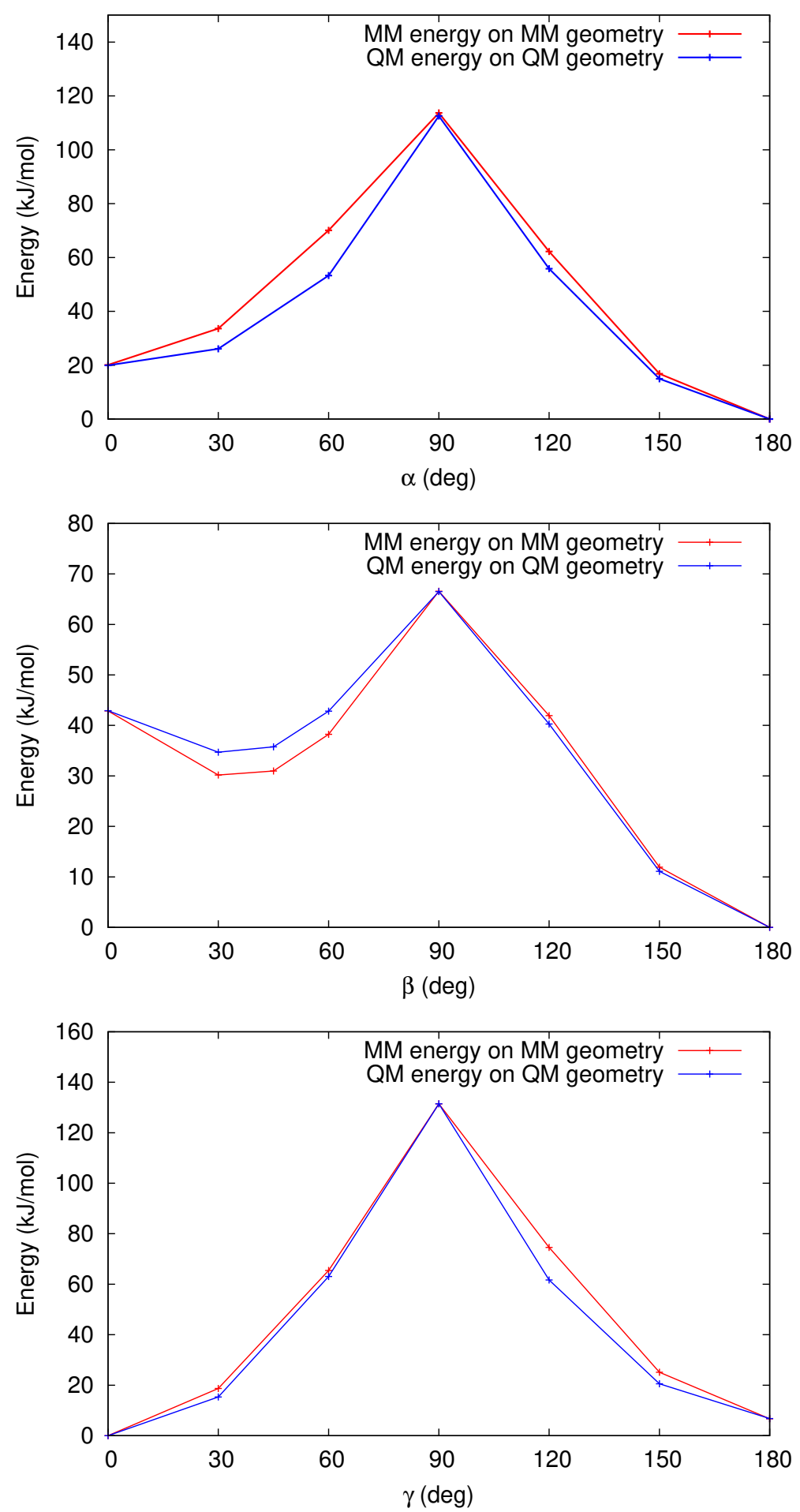

Figure 5.16: Potential energy profiles obtained with a constrained geometry optimization at the QM B3LYP/6-311+G(2d,p) and MM levels. 


\section{Bibliography}

[1] H. N. Motlagh, J. O. Wrabl, J. Li, and V. J. Hilser, Nature 508, 331 (2014).

[2] A. W. Fenton, Trends Biochem. Sci. 33, 420 (2008).

[3] D. Kern and E. R. Zuiderweg, Curr. Opin. Struc. Biol. 13, 748 (2003).

[4] C.-J. Tsai, A. Del Sol, and R. Nussinov, J. Mol. Biol. 378, 1 (2008).

[5] A. C. M. Ferreon, J. C. Ferreon, P. E. Wright, and A. A. Deniz, Nature 498, 390 (2013).

[6] J.-P. Changeux, Nat. Rev. Mol. Cell Biol. 14, 819 (2013).

[7] J. H. Choi, A. H. Laurent, V. J. Hilser, and M. Ostermeier, Nat. Commun. 6, 6968 (2015).

[8] R. A. Brown, V. Diemer, S. J. Webb, and J. Clayden, Nat. Chem. 5, 853 (2013).

[9] G. Y. Tonga, Y. Jeong, B. Duncan, T. Mizuhara, R. Mout, R. Das, S. T. Kim, Y.-C. Yeh, B. Yan, S. Hou, and V. M. Rotello, Nat. Chem. 7, 597 (2015).

[10] W. Szymański, J. M. Beierle, H. A. Kistemaker, W. A. Velema, and B. L. Feringa, Chem. Rev. 113, 6114 (2013).

[11] A. A. Beharry and G. A. Woolley, Chem. Soc. Rev. 40, 4422 (2011).

[12] R. Klajn, Chem. Soc. Rev. 43, 148 (2014).

[13] D. P. Nguyen, M. Mahesh, S. J. Elsässer, S. M. Hancock, C. Uttamapinant, and J. W. Chin, J. Am. Chem. Soc. 136, 2240 (2014).

[14] J. Broichhagen and D. Trauner, Curr. Opin. Chem. Biol. 21, 121 (2014).

[15] R. M. Putri, J. W. Fredy, J. J. Cornelissen, M. S. Koay, and N. Katsonis, Chem. Phys. Chem. 17, 1815 (2016).

[16] J. R. Kumita, O. S. Smart, and G. A. Woolley, Proc. Natl. Acad. Sci. 97, 3803 (2000).

[17] G. A. Woolley, Acc. Chem. Res. 38, 486 (2005). 
[18] B. Schierling, A.-J. Noël, W. Wende, E. Volkov, E. Kubareva, T. Oretskaya, M. Kokkinidis, A. Römpp, B. Spengler, and A. Pingoud, Proc. Natl. Acad. Sci. 107, 1361 (2010).

[19] M. Aizawa, K. Namba, and S. Suzuki, Arch. Biochem. Biophys. 182, 305 (1977).

[20] M. Bose, D. Groff, J. Xie, E. Brustad, and P. G. Schultz, J. Am. Chem. Soc. 128, 388 (2006).

[21] G. A. Woolley, A. S. Jaikaran, M. Berezovski, J. P. Calarco, S. N. Krylov, O. S. Smart, and J. R. Kumita, Biochemistry 45, 6075 (2006).

[22] A. Koçer, M. Walko, W. Meijberg, and B. L. Feringa, Science 309, 755 (2005).

[23] M. R. Banghart, M. Volgraf, and D. Trauner, Biochemistry 45, 15129 (2006).

[24] I. Tochitsky, M. R. Banghart, A. Mourot, J. Z. Yao, B. Gaub, R. H. Kramer, and D. Trauner, Nat. Chem. 4, 105 (2012).

[25] S. Pittolo, X. Gómez-Santacana, K. Eckelt, X. Rovira, J. Dalton, C. Goudet, J.-P. Pin, A. Llobet, J. Giraldo, A. Llebaria, and P. Gorostiza, Nat. Chem. Biol. 10, 813 (2014).

[26] M. Volgraf, P. Gorostiza, S. Szobota, M. R. Helix, E. Y. Isacoff, and D. Trauner, J. Am. Chem. Soc. 129, 260 (2007).

[27] M. Schönberger and D. Trauner, Angew. Chem. Int. Ed. 53, 3264 (2014).

[28] M. Volgraf, P. Gorostiza, R. Numano, R. H. Kramer, E. Y. Isacoff, and D. Trauner, Nat. Chem. Biol. 2, 47 (2006).

[29] D. Strickland, K. Moffat, and T. R. Sosnick, Proc. Natl. Acad. Sci. 105, 10709 (2008).

[30] P. Ascenzi and M. Fasano, Biophys. Chem. 148, 16 (2010).

[31] G. Fanali, A. di Masi, V. Trezza, M. Marino, M. Fasano, and P. Ascenzi, Mol. Aspects Med. 33, 209 (2012).

[32] F. Yang, C. Bian, L. Zhu, G. Zhao, Z. Huang, and M. Huang, J. Struct. Biol. 157, 348 (2007).

[33] G. Li, D. Magana, and R. B. Dyer, Nat. Commun. 5, 3100 (2014).

[34] M. N. Khan, J. Pharm. Sci. 73, 1767 (1984).

[35] R. M. Putri, Optical Control over Monomeric and Multimeric Protein Hybrids (University of Twente, 2017).

[36] G. Bianco, S. Forli, D. S. Goodsell, and A. J. Olson, Protein Sci. 25, 295 (2016). 
[37] G. M. Morris, R. Huey, W. Lindstrom, M. F. Sanner, R. K. Belew, D. S. Goodsell, and A. J. Olson, J. Comput. Chem. 30, 2785 (2009).

[38] W. Humphrey, A. Dalke, and K. Schulten, J. Mol. Graph. 14, 33 (1996).

[39] D. Van Der Spoel, E. Lindahl, B. Hess, G. Groenhof, A. E. Mark, and H. J. Berendsen, J. Comput. Chem. 26, 1701 (2005).

[40] R. B. Best and G. Hummer, J. Phys. Chem. B 113, 9004 (2009).

[41] K. Lindorff-Larsen, S. Piana, K. Palmo, P. Maragakis, J. L. Klepeis, R. O. Dror, and D. E. Shaw, Proteins 78, 1950 (2010).

[42] J. Wang, R. M. Wolf, J. W. Caldwell, P. A. Kollman, and D. A. Case, J. Comput. Chem. 25, 1157 (2004).

[43] G. Vriend, J. Mol. Graphics 8, 52 (1990).

[44] T. J. Dolinsky, J. E. Nielsen, J. A. McCammon, and N. A. Baker, Nucleic Acids Res. 32, W665 (2004).

[45] S. Miyamoto and P. A. Kollman, J. Comput. Chem. 13, 952 (1992).

[46] T. Darden, D. York, and L. Pedersen, J. Chem. Phys. 98, 10089 (1993).

[47] G. Bussi, D. Donadio, and M. Parrinello, J. Chem. Phys. 126, 014101 (2007).

[48] M. Parrinello and A. Rahman, J. Appl. Phys. 52, 7182 (1981).

[49] B. Hess, H. Bekker, H. J. Berendsen, and J. G. Fraaije, J. Comput. Chem. 18, 1463 (1997).

[50] G. Morra, G. Verkhivker, and G. Colombo, PLOS. Comput. Biol. 5, e1000323 (2009).

[51] R. Ambrosetti, R. Bianchini, S. Fisichella, M. Fichera, and M. Zandomeneghi, Chem. Eur. J. 2, 149 (1996).

[52] L. Yang, A. Liu, S. Cao, R. M. Putri, P. Jonkheijm, and J. J. Cornelissen, Chem. Eur. J. 22, 15570 (2016).

[53] P. Cieplak, W. D. Cornell, C. Bayly, and P. A. Kollman, J. Comput. Chem. 16, 1357 (1995).

[54] M. J. Frisch, G. W. Trucks, H. B. Schlegel, G. E. Scuseria, M. A. Robb, J. R. Cheeseman, G. Scalmani, V. Barone, B. Mennucci, G. A. Petersson, H. Nakatsuji, M. Caricato, X. Li, H. P. Hratchian, A. F. Izmaylov, J. Bloino, G. Zheng, J. L. Sonnenberg, M. Hada, M. Ehara, K. Toyota, R. Fukuda, J. Hasegawa, M. Ishida, T. Nakajima, Y. Honda, O. Kitao, H. Nakai, T. Vreven, J. A. Montgomery, Jr., J. E. Peralta, F. Ogliaro, M. Bearpark, J. J. Heyd, E. Brothers, K. N. 
Kudin, V. N. Staroverov, R. Kobayashi, J. Normand, K. Raghavachari, A. Rendell, J. C. Burant, S. S. Iyengar, J. Tomasi, M. Cossi, N. Rega, J. M. Millam, M. Klene, J. E. Knox, J. B. Cross, V. Bakken, C. Adamo, J. Jaramillo, R. Gomperts, R. E. Stratmann, O. Yazyev, A. J. Austin, R. Cammi, C. Pomelli, J. W. Ochterski, R. L. Martin, K. Morokuma, V. G. Zakrzewski, G. A. Voth, P. Salvador, J. J. Dannenberg, S. Dapprich, A. D. Daniels, Ö. Farkas, J. B. Foresman, J. V. Ortiz, J. Cioslowski, and D. J. Fox, Gaussian 09 Revision D.01, gaussian Inc. Wallingford CT 2009.

[55] R. D. Amos and R. Kobayashi, Mol. Phys. 113, 1674 (2015).

[56] Y. Myung and S. Han, Bull. Korean Chem. Soc. 31, 2581 (2010).

[57] P. Duchstein, C. Neiss, A. Görling, and D. Zahn, J. Mol. Model. 18, 2479 (2012). 


\section{Summary}

\section{Computational exploration of optical and functional proper- ties of biorelevant photoswitches}

Developing molecular photoswitches for applications in material science and biology is a very active field of research in chemistry. Photoswitches are a class of small molecular machines whose structures and properties can be switched between two or more states with light as a trigger. The importance of this field is marked by the awarding of the Nobel Prize in Chemistry in 2016 "for the design and synthesis of molecular machines". In this dissertation, we contribute to the better understanding of three selected photoswitches using computational means. These photoswitches are biorelevant because they either exist in nature (commonly called chromophores) or can be covalently coupled to a biomolecular support and operated at physiological conditions.

When a photoswitch is irradiated with light at a particular wavelength, the photon energy is absorbed to promote electronic transition from an occupied to an unoccupied orbital yielding an electronic excited state. The excited state of an isomer should relax to the ground state of another isomer for a successful transformation. In Chapter 3, we develop a new class of many-body wave functions within the accurate quantum Monte Carlo (QMC) framework to explore the excited-state potential energy surfaces (PES) of photoswitches. In constructing these wave functions, we take advantage of the flexibility of QMC methods that can work with multiple sets of localized active orbitals corresponding to the different bonding pattern of the dominant Lewis resonance structures of the molecule. We also adopt the concept of orbitals domains of local coupled-cluster methods to select orbitals to correlate within an active space. The determinants are built from the domains, grouped in classes, and systematically included in the wave-function expansion. Using retinal model chromophores as prototypical test cases, we find that our novel and compact wave functions can be flexibly and accurately applied to very different parts of a PES using the same, small set of determinants. Therefore, our multi-resonance local wave functions together with the recently developed scheme for the efficient computation of interatomic forces open the possibility to efficiently compute the excited-state PES of the full retinal chromophore as well as other large photoswitches at the QMC level.

In Chapter 4, we embark on a journey to understand the complex molecular photoswitching mechanism of donor-acceptor Stenhouse adducts (DASAs). These photoswitches are relatively new, and we believe that the effective and efficient de- 
velopment of these systems can only be achieved via a thorough understanding of the reaction mechanism in different environments. At the density functional theory level, we are able to explain the available experimental data of photoswitching of two relevant DASA molecules in a nonpolar (toluene) and a polar (methanol) solvent as regards the reversibility of the reactions, the relative rate of the ring-closure reaction in the two contrasting solvents, and the different structures of the final products. We also present a preliminary excited-state study in vacuum with multiconfigurational wave-function methods. We find that computing the excited-state PES of these systems requires a method that can account for both static and dynamical electron correlation. Furthermore, based on the existence of an excited-state minimum computed at a higher level of (perturbation-based) theory, we infer the presence of a barrier for rotation around any formal double bonds.

Finally, while the current knowledge of the reaction mechanism of spiropyran (SP) photoswitches appears to be sufficient as demonstrated by their application in many different fields, we focus our attention on understanding the very recent use of SP for drug-delivery applications in Chapter 5. Our experimental collaborators have demonstrated that, by photoswitching a single unit of spiropyran covalently connected to subdomain IA of the human serum albumin protein, one induces ligand release in the adjacent subdomain IB. Our molecular dynamics simulations elucidate the nature of the interactions among the protein, the photoswitch and the ligand. We find that the covalent attachment of SP weakens a key hydrogen bonds maintaining the ligand in the IB binding pocket, and that further perturbation is introduced by photoswitching SP. Furthermore, our internal dynamics analysis of the two subdomains reveals that, upon photoswitching, the binding pocket breathes more, and that the motion of the two subdomains become even more decoupled. Our simulations demonstrate the allosteric nature of the interaction between the two subdomains induced by the presence of the photoswitch and provide an explanation of the factors which regulate the ligand release observed experimentally. 


\section{Samenvatting}

\section{Computationele verkenning van optische en funtionele eigen- schappen van biorelevante fotoswitches}

De ontwikkeling van moleculaire fotoswitches voor toepassingen in materiaalwetenschap en biologie is een zeer actief veld van research. Fotoswitches is een klasse kleine moleculaire machines waarvan de structuur en eigenschappen met behulp van licht tussen twee of meer toestanden kunnen worden geschakeld. De belangrijkheid van dit veld wordt gemarkeerd door de toekenning van de Nobelprijs voor Scheikunde in 2016 "voor het ontwerp en de synthese van moleculaire machines". Met een computationele aanpak dragen we in dit proefschrift bij tot een beter begrip van drie geselecteerde fotoswitches. Deze fotoswitches zijn biorelevant omdat ze in de natuur voorkomen (gewoonlijk chromoforen genoemd) of ze kunnen covalent gebonden zijn aan een biomoleculair systeem en worden gebruikt onder fysiologische omstandigheden.

Wanneer een fotoswitch wordt bestraald met licht van een bepaalde golflengte, leidt de geabsorbeerde foton energie tot een elektronische overgang van een bezette naar een onbezette orbitaal. De aangeslagen toestand die dit oplevert kan leiden tot een succesvolle transformatie wanneer relaxatie optreedt naar een grondtoestand van een andere isomeer. In Hoofdstuk 3 ontwikkelen we een nieuwe klasse veeldeeltjes golffuncties binnen het kader van de nauwkeurige quantum Monte Carlo (QMC) methoden om het potentiele energie oppervlak (PES) van de aangeslagen toestanden van fotoswitches te verkennen. Bij de constructie van deze golffunties maken we gebruik van de flexibiliteit van de QMC methoden. Meerdere sets gelokaliseerde actieve orbitals kunnen worden gebruikt welke overeenkomen met verschillende bindingspatronen van dominante Lewis resonantie structuren van het molecule. We gebruiken het concept orbitaal domein uit lokale coupled-cluster methoden om orbitalen te selecteren die correleren binnen de actieve ruimte. De determinanten zijn opgebouwd uit in klassen gegroepeerde orbitaal domeinen en zijn zo systematisch onderdeel van de golffunctie expansie. Met chromoforen uit het retina model als test cases zien we dat onze nieuwe en compacte golffuncties flexibel en nauwkeurig zijn wanneer ze toegepast worden op verschillende delen van een PES wanneer we dezelfde kleine set determinanten gebruiken. Daarom bieden onze multi-resonantie lokale golffuncties, samen met het recent ontwikkelde schema voor efficiente berekening van interatomische krachten, een opening voor de mogelijk 
efficiente berekening van de aangeslagen toestand PES van het volledige retina chromofoor, alsmede andere grote fotoswitches, op het QMC niveau.

In Hoofdstuk 4 beginnen we een reis om het complexe moleculaire fotoswitching mechanisme te begrijpen van donor-acceptor Stenhouse adducts (DASAs). Deze fotoswitches zijn relatief nieuw. We geloven dat de effectieve en efficiënte ontwikkeling van deze systemen alleen mogelijk is met het begrip van de reactie mechanismen in verschillende omgevingen. Op het niveau van dichtheidsfunctionaaltheorie zijn we in staat om de beschikbare experimentele gegevens te verklaren van de fotoswitching van twee relevante DASA moleulen in een niet polair (tolueen) en een polair (methanol) oplosmiddel, met betrekking tot de reversibiliteit van de reacties, de relatieve snelheid van de ringsluiting reatie in de twee contrasterende oplosmiddelen en de verschillende structuren van de uiteindelijke producten. We presenteren ook een voorlopige studie naar aangeslagen toestanden in vacuüm met multi-configurationele golffunctie methoden. We vinden dat de berekening van de aangeslagen toestand PES van deze systemen een methode vereist die zowel statische als dynamische correlatie in rekening brengt. Op basis van het bestaan van een aangeslagen toestand, berekend op een hoger niveau van (storingsrekening gebaseerde) theorie, leiden we de aanwezigheid af van een rotatiebarrière rond formele dubbele bindingen.

Ten slotte, terwijl de huidige kennis van het reactie mechanisme van spiropyran (SP) fotoswitches al voldoende goed blijkt te zijn zoals aangetoond door toepassingen in veel verschillende velden, focussen we onze aandacht op het begrip van het zeer recente gebruik van SP voor toepassingen van geneesmiddelafgifte in Hoofdstuk 5. Onze experimentele collega's hebben aangetoond dat, door het fotoswitchen van een enkel spiropyran eenheid dat covalent gebonden is aan subdomein IA van het menselijk serum albumine-eiwit, men ligand-afgifte kan induceren in het naastgelegen subdomein IB. Met onze moleculaire dynamica simulaties ontrafelen we de aard van de interacties tussen de proteïnen, de fotoswitch en het ligand. We vinden dat de covalente binding van SP belangrijke waterstof bindingen verzwakt waarmee het ligand in het IB centrum gebonden is en dat verdere verstoring wordt geïntroduceerd door het fotoswitchen van SP. Bovendien laat onze interne dynamica analyse van de twee subdomeinen zien dat het geometrie van het bindingscentrum meer fluctueert en dat de beweging van de twee subdomeinen bij fotoswitching meer ontkoppeld raakt. Onze simulaties tonen aan dat de allosterische karakter van deze interacties tussen de twee subdomeinen geïnduceerd worden door de aanwezigheid van de fotoswitch en bieden een verklaring voor de experimenteel waargenomen factoren die de ligandafgite reguleren. 


\section{List of publications}

- H. Zulfikri, C. Amovilli and C. Filippi,

"Multiple-resonance local wave functions for accurate excited states in quantum Monte Carlo", J. Chem. Theory Comput. 12, 1157 (2016). (Chapter 3)

- R. Guareschi, H. Zulfikri, C. Daday, F. M. Floris, C. Amovilli, B. Mennucci and C. Filippi,

"Introducing QMC/MMpol: Quantum Monte Carlo in polarizable force fields for excited states",

J. Chem. Theory Comput. 12, 1674 (2016).

- R. Alessandri, H. Zulfikri, J. Autschbach and H. Bolvin, "Crystal field in rare-earth complexes: From electrostatics to bonding", Chem. Eur. J. 24, 1 (2018).

This publication was selected by the editors as a "hot paper".

- R. M. Putri, H. Zulfikri, J. W. Fredy, A. Juan, P. Tananchayakul, J. J. L. M. Cornelissen, M. S. T. Koay, C. Filippi and N. Katsonis, "Photo-programming allostery in the human serum albumin", accepted on Bioconjugate Chemistry. (Chapter 5)

The first two authors contributed equally to this publication.

- H. Zulfikri and C. Filippi, "Investigating the thermal step of photoswitching mechanism of donor-acceptor Stenhouse adducts: Electrocyclization and subsequent forking tautomerization pathways", in preparation. (Chapter 4)

- H. Zulfikri and C. Filippi, "On the actinic step of donor-acceptor Stenhouse adduct photoswitch: A CASSCF and CASPT2 study", in preparation. (Chapter 4) 


\section{Acknowledgements}

With the end of this dissertation, I would like to express my gratitude to all the people who have supported me during my PhD life at the University of Twente. First of all, I would like to thank my promotor, Prof. Claudia Filippi, for a very close and constant supervision and help toward personal problems (e.g. all the administrative headaches which followed my road accident). I have learned a lot from her during the past four and a half years about practicing good science, scientific writing and maintaining fruitful collaborations with other research groups. I am also grateful that I have been given opportunities to communicate my research and broaden my knowledge in many scientific conferences in Europe and the USA.

My scientific research has also been possible thanks to the help of very good collaborators. I thank Prof. Claudio Amovilli from the University of Pisa for helping me in the design of the multi-resonance wave functions and for his guidance during my research visit in 2015. The remaining two-third of my work has been performed in close collaborations with experimental colleagues. I thank Dr. Rindia M. Putri and Prof. Nathalie Katsonis from the University of Twente for fruitful scientific discussions on the interplay between simulations and experiments in our protein-hybrid work. I thank Dr. Michael M. Lerch and Dr. Wiktor Szymański from the University of Groningen, Dr. Mariangela Di Donato from the European Laboratory for NonLinear Spectroscopy in Florence, Mark Koenis and Prof. Wybren Jan Buma from the University of Amsterdam, for the many exciting discussions and data exchanges that helped to shape the direction of my work on DASA photoswitches.

I realize that being in a good work environment greatly advances one's career and success. Therefore, I would like to thank the (former) members of the CCP group. Dr. Csaba Daday, thank you for introducing me to life in Enschede, accompanying me to the hospital when I got hit by a car and helping with computational problems and codes. Dr. Riccardo Guareschi, thank you for your advices and for being a helpful colleague. Monika Dash, thank you for inviting me and my wife for a nice Indian dinner and also for our great conversations and your help. I also want to thank Prof. Wim Briels, Dr. Wouter den Otter, Dr. Barry Fitzgerald, Dr. Ioana Illie, Dr. Matteo Giani, Dr. Vishal Ahuja and Vishal Metri, for our delightful lunches, conversations and group outings. To Monika Dash and Vishal Metri, I wish smooth $\mathrm{PhD}$ journeys with glory endings. Els Braker-Peerik, thank you for your help with all the documents and paperwork needed during these years.

While preparing for the $\mathrm{PhD}$ defense, I also got help from my friends. I thank Dr. Gerrit-Jan Linker for providing the Dutch text for the summary of this dissertation. 
To my paranymph: Monika Dash and Debby Fajar Mardhian, thank you for your help before, during and after the academic ceremony of my $\mathrm{PhD}$ defense.

During my stay in Enschede, I am grateful that I have met old friends and made new friends who were also studying here, and that we helped each other. I thank the Indonesian community for giving me the opportunity to participate in the association and organize scientific and cultural events. For those who are still in the middle of their study, I sincerely wish you all the best in finalizing your degree. I also thank the Indonesian-Dutch families in Enschede who have been very kind to my family. I would like to also express my gratitude to the UT Muslim community who has made practicing religion at UT as easy as at my previous university in my own country.

Finally and especially, I would like to thank my big families for their constant support and encouragement. I thank my "squishy" wife, Rahma Muthia, who always stood by me, supported and counseled me in every way. I am so blessed to have married you in 2015 and, ever since, we walk through happy and difficult times together in our lives and $\mathrm{PhD}$ journeys. The arrival of our "squishy" son, Luqman Mochtar Habiburrahman, in mid-2017 has also colored our adventures in this country. I hope that I have dedicated sufficient quality times to both of you, particularly in the last busiest months. Teruntuk kedua Mama dan Papa, Husna, uda Hendra, dan uda Iki, Aan sangat berterima kasih atas semua dukungannya. Alhamdulillaah perjalanan S3 ini berakhir dengan baik. Keberkahan ilmu dan perjalanan selanjutnya merupakan pinta yang mohon didoakan kepada yang Maha Kuasa. 
